\title{
Plastische Berechnung von Stahlbetonscheiben und -balken
}

\section{Working Paper}

Author(s):

Müller, Peter

Publication date:

1978

Permanent link:

https://doi.org/10.3929/ethz-a-000147836

Rights / license:

In Copyright - Non-Commercial Use Permitted

Originally published in:

Bericht / Institut für Baustatik und Konstruktion ETH Zürich 83 


\section{Plastische Berechnung von \\ Stahlbetonscheiben und -balken}

Peter Müller 


\title{
Plastische Berechnung von Stahlbetonscheiben und -balken
}

\author{
von \\ Dr. sc. techn. Peter Müller \\ Institut für Baustatik und Konstruktion \\ Eidgenössische Technische Hochschule Zürich
}


Die Arbeit ist ein weiterer Beitrag unseres Institutes zur Anwendung der Plastizitätstheorie für die Bestimmung des Tragwiderstandes von Stahlbetonträgern unter Biegung, Schub und Torsion. Neben der Klärung fundamentaler Fragen zeigt sie auch Lösungen für praktische Probleme auf und behandelt daraus folgende konstruktive Details.

Unsere langjährigen, beharrlichen Forschungen auf diesem Gebiet haben in dieser als Doktorarbeit verfassten Studie und in früheren Berichten ihren Niederschlag gefunden. Mit Freude dürfen wir feststellen, dass sich daraus eine einheitliche, auf der Plastizitätstheorie fundierte Berechnungsmethode zur Bestimmung des Tragwiderstandes von Stahlbetonträgern entwickelt hat, die sowohl in den Normen als auch in der Praxis zunehmend Anwendung findet. 
Vorwort

1. EINLEITUNG

1.1 Zielsetzung und Uebersicht

1.2 Grundlagen

1.3 Grenzwertsätze der Plastizitätstheorie [5]

K A P I T E L I: FLIESSBEdINGUng UND FLIESSGESETZ FUER STAHLBETONSCHEIBEN

2. FLIESSBEDINGUNGEN

2.1 Annahmen

2.2 Herleitung

2.3 Statik der einzelnen Fliessregimes

3. FLIESSGESETZ UND KINEMATIK 11

3.1 Spannungs-Verzerrungs-Beziehungen 12

3.2 Kinematik der einzelnen Fliessregimes $\quad 12$

$\begin{array}{ll}3.3 \text { Unstetige Geschwindigkeitsfelder } & 15\end{array}$

4. ERGAENZUNGEN $r$

4.1 Nichtorthogonale Armierung 19

4.2 Wirklichkeitsnahere zweiaxiale Betonfestigkeit 22

5. EXPERIMENTELLE ERGEBNISSE 25

$\begin{array}{lllll}K & \text { A P I TE L II: BALKENTHEORIE } & 27\end{array}$

6. BALKEN IM FLIESSREGIME I: FACHWERKMODELL UND BALKENTHEORIE 27

6.1 Parametrische Darstellung von Fliessfläche und Fliessgesetz im Regime I 27

6.2 Fachwerkmodell ! 29

6.3 Balkentheorie $\quad 32$

6.4 Kinematisch zulässige verträgliche Geschwindigkeitsfelder in der Stegscheibe 39

6.5 Zusammenfassung der Resultate 45

7. BALKEN IN DEN FLIESSREgIMES III, IV, V: OBERE SCHUBSPANNUNGSGRENZE 47

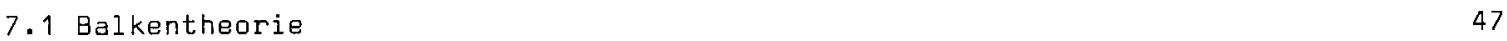

7.2 Kinematisch zulässige verträgliche Geschwindigkeitsfelder der Stegscheibe

$\begin{array}{ll}7.3 \text { Vergleich mit Versuchsresultaten und Normen } & 57\end{array}$

8. BALKEN IM FLIESSREgIME I: BIEGUNG UND TORSION 68

8.1 Bisherige plastizitätstheoretische Ansätze 68

8.2 Kollapsmechanismus für Balken mit Rechteckquerschnitt 73

8.3 Kollapsmechanismus für Balken mit polygonalem Querschnitt 76

8.4 Balkentheorie $\quad 79$

8.5 Ergänzungen $\quad 85$ 
9. Plastizitaetstheorie der stamlbetonscheibe 94

9.1 Spannungsfelder im Regime I 94

9.2 Geschwindigkeitsfelder im Regime I 99

9.3 Spannungsfelder in den Regimes III-VII 103

9.4 Geschwindigkeitsfelder in den Regimes III-VII 105

$\begin{array}{ll}\text { 9.5. Ergänzungen } & 107\end{array}$

10. ZUR SCHUBBEMESSUNG VON STAHLBETONBALKEN 110

10.1 Träger unter verteilter Belastung 110

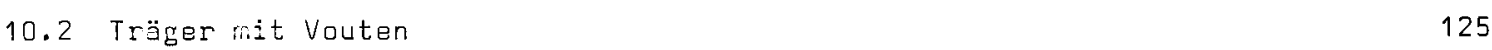

10.3 Krafteinleitungen $\quad \cdot 125$

11. FALLSTUDIE "SHEAR WALL COUPLING BEAM 391" [44]

11.1 Problemstellung 132

11.2 Direkte Schubübertragung (Sprengwerkwirkung) 134

11.3 Schubübertragung durch Bügel allein 138

11.4 Gemischte Schubübertragung 142

12. SCHLUSSWORT UND ZUSAMMENFASSUNG 145

12.1 Schlusswort 145

$\begin{array}{ll}12.2 \text { Zusammenfassung } & 145\end{array}$

12.3 Abstract 146

$\begin{array}{lll}12.4 & \text { Resumé } & 147\end{array}$

$\begin{array}{lr}\text { LITERATURVERZEICHNIS } & 148\end{array}$

BEZEICHNUNGEN $\quad 151$

ANHANG A: Ableitung der Fliessbedingungen (2.7) aus ihrer Stützfunktion (2.5) 155

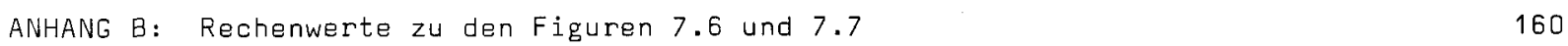




\section{EINLEITUNG}

\subsection{Zielsetzung und Uebersicht}

Der wirklichkeitsnahen Erfassung des Bruchzustandes von Stahlbetontragwerken kommt heute zentrale Bedeutung zu. Hat man sich bisher damit begnügt, den Nachweis ausreichender Bemessung durch den Vergleich vorhandener Spannungen im Gebrauchszustand mit zulässigen Spannungen zu führen, so drängt sich heute eine differenziertere Betrachtungsweise auf, sollen Fortschritte hinsichtlich besserer Materialausnützung und zuverlässigerem Tragverhalten erzielt werden. Die neueste Entwicklung in den Stahlbetonnormen basiert auf der Bemessung auf Grenzzustände. Diese sind im wesentlichen der Verlust der Nutzungsfähigkeit infolge unzulässiger Deformationen und Rissebildung unter Gebrauchslast einerseits und der Verlust der Tragfähigkeit andererseits.

Für die Erfassung des zweiten Grenzzustandes mit vertretbarem Rechenaufwand hat sich die Theorie ideal-plastischer Körper als wertvolles Hilfsmittel erwiesen. Ueber die plastische Berechnung der Biegetragfähigkeit von Stahlbetonplatten und Stahlbetonbalken existiert umfangreiche Literatur. Wesentliche Impulse gingen von der Plastizitätstheorie auf die Erfassung und das Verständnis der Schubtragfähigkeit von Stahlbetonbalken aus. In den experimentellen und theoretischen Arbeiten [34 - 36, 18 - 23] wurde geżigt, dass das bereits von Ritter und Mörsch eingeführte Fachwerkmodell die Tragfähigkeit von unterarmierten Stahlbetonbalken unter Torsion, Biegung und Querkraft gut zu erfassen vermag, wenn es mit den Methoden der Plastizitätstheorie kombiniert wird. Das Modell bildet deshalb heute in verschiedenen Stahlbetonnormen die rationale Grundlage für die Bruchbemessung der Armierung [25, 29, 30].

Die theoretischen Grundlagen der mit dem Fachwerkmodell gewonnenen Querschnittsinteraktionsbeziehungen sind jedoch noch nicht vollständig. Plastizitätstheoretisch strenge obere Grenzwerte und Kollapsmechanismen sind bisher noch nicht angegeben worden. Die Kinematik plastifizierter Schubwände lässt sich aber nur mit einem Scheibenansatz beschreiben. Schubübertragung durch Sprengwerk- oder Bogenwirkung lässt sich mit Querschnittsinteraktionsbeziehungen nicht erfassen und stellt für Träger mit dünnen Stegen ein typisches Scheibenproblem dar. Obschon Fliessbedingungen für die Stahlbetonscheibe seit langem bekannt sind [15], ist die Plastizitätstheorie jedoch erst in einigen wenigen Arbeiten auf Stahlbetonscheiben und wandartige Träger angewandt worden [26].

Hier will die vorliegende Arbeit einen Beitrag leisten. Die Zielsetzung weist drei Schwerpunkte auf. Den ersten. Schwerpunkt bildet die Weiterentwicklung der theoretischen Grundlagen der plastischen Berechnung von unterarmierten, dünnwandigen Stahlbetonbalken unter Torsion, Biegung und Querkraft (Abschnitte 6 und 8). Dieser Teil der Arbeit konzentriert sich vor allem auf die mit dem Konzept Makromodell Fachwerk nicht erfassbaren Kollapsmechanismen.

In den erwähnten Arbeiten [18 - 23] wurde immer vorausgesetzt, dass der Kollaps durch Fliessen der Armierung eingeleitet wird. Den zweiten Schwerpunkt bildet deshalb das Studium der Frage, ob mit der Plastizitätstheorie auch Schubbrüche erfasst werden können, bei welchen der Kollaps durch das Versagen des Stegbetans eingeleitet wird (Abschnitt 7). Diese Frage berührt insbesondere das Problem der oberen Grenze für die nominelle Stegschubspannung von Stahlbetonbalken.

Den dritten Schwerpunkt schliesslich bilden die Diskussion der allgemeinen Spannungs- und Geschwindigkeitsfelder der plastifizierten Stahlbetonscheibe (Abschnitt 9) und die Anwendung 
der Ergebnisse auf spezielle Schubprobleme in Stahlbetonträgern (Abschnitt 10) unf auf wandartige Träger (Abschnitt 11). Fragen, auf die in Abschnitt 10 eingegangen wird, sind: Garantiert die querschnittsweise Bemessung von Stahlbetonträgern aufgrund des Fachwerkmodells die Existenz stabiler, statisch zulässiger Scheibenspannungszustände im Steg, wenn die Querkraft variiert oder die zur Dimensionierung gewählte Diagonalenneigung gewechselt wird (Abschnitt 10.1), wenn Vouten vorhanden sind (Abschnitt 10.2) oder wenn konzentrierte Lasten eingeleitet werden (Abschnitt 10.3)? Die plastische Berechnung eines wandartigen Trägers zeigt insbesondere den Einfluss der Sprengwerkwirkung auf.

Die Grundlage für die Behandlung der erwähnten Probleme bilden die Ableitung von Fliessbedingungen für die Stahlbetonscheibe und die Diskussion der Statik und Kinematik der einzelnen Fliessregimes (Abschnitte 1 bis 5 ).

1.2 Grundlagen

Grundlage der vorliegenden Arbeit ist die Plastizitätstheorie [1 - 6], insbesondere die Theorie des plastischen Potentials und die Grenzwertsätze. Letztere sind unter folgenden Annahmen gültig:
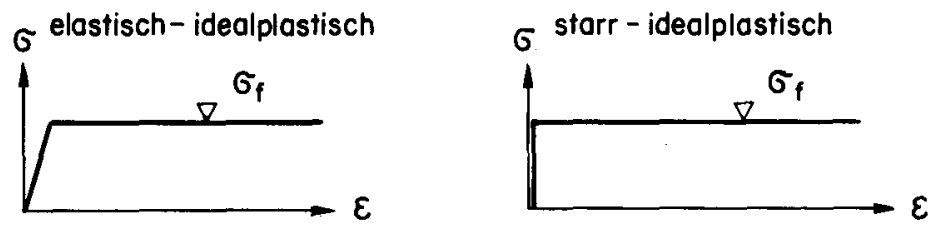

Fig. 1.1 Idealisiertes Spannungs - Dehnungs Diogramm

1. Ein starr-idealplastisches oder elastisch-idealplastisches Materialverhalten wird vorausgesetzt (Fig. 1.1).

2. Die bis zum Erreichen der Traglast eintretenden elastischen und plastischen Formänderungen sind klein gemessen an den Dimensionen des Systems, so dass das Gleichgewicht am undeformierten system formuliert werden kann.

3. Die Belastung wird statisch aufgebracht und proportional gesteigert.

Unter Berücksichtigung von Punkt 2 ist es für die Traglastberechnung irrelevant, ob starridealplastisches oder elastisch-idealplastisches Materialverhalten vorausgesetzt wird.

Die Annahme idealplastischer Spannungs-Dehnungs-Beziehungen stellt eine beträchtliche Idealisierung des tatsächlichen Materialverhaltens dar. Es ist heute durchaus möglich, auf so einschränkende Annahmen zu verzichten. Mit Hilfe von auf der Methode der finiten Elemente basierenden Computerprogrammen, die nichtlineares Materialverhalten, Bruchkriterien und Rissebildung berücksichtigen, können Stahlbetontragwerke über den ganzen Lastbereich ziemlich wirklichkeitsnah erfasst werden, z.B. [45, 46]. Die Genauigkeit, mit der ein Modell die Wirklichkeit erfassen kann, stellt jedoch nur einen der Aspekte dar, die eine Modellwahl beeinflussen. Ein weiterer wesentlicher Gesichtspunkt ist der Zweck, dem ein Modell dienen soll [14].

Die erwähnten Computerprogramme sind für eine routinemässige Anwendung in der Praxis heute noch zu kompliziert und kostspielig. Sie dienen vor allem Forschungszwecken. Ihr Einsatz kann 
die Anzahl der durchzuführenden Versuche reduzieren, die Ableitung einfacher Bemessungsregeln wird dadurch im allgemeinen aber kaum erleichtert.

Das idealplastische Modeli idealisiert dagegen die Wirklichkeit wesentlich stärker. Dank seiner Einfachheit eignet es sich aber gut als rationale Basis zur Gewinnung einfacher Bemessungsregeln für Stahlbetonnormen.

\subsection{Grenzwertsätze der Plastizitätstheorie}

\section{Statischer Grenzwertsatz}

"Jede Belastung, zu der sich ein stabiler, statisch zulässiger Spannungszustand angeben lässt, liegt nicht höher als die Traglast."

Ein Spannungszustand heisst statisch zulässig, wenn er überall die Gleichgewichtsbedingungen und die statischen Randbedingungen erfüllt. Er ist stabil, wenn die fliessbedingungen nirgends überschritten werden.

\section{Kinematischer Grenzwertsatz}

"Jede Belastung, zu der sich ein instabiler, kinematisch zulässiger Bewegungszustand angeben lässt, liegt nicht tiefer als die Traglast."

Ein Bewegungszustand wird als kinematisch zulässig bezeichnet, wenn er die kinematischen Bindungen und die kinematischen Randbedingungen des Systems erfüllt. Er heisst instabil, wenn die Leistung der äusseren Lasten grösser oder gleich der Dissipationsleistung ist.

\section{Verträglichkeitssatz}

"Lassen sich für eine Belastung $P$ ein stabiler, statisch zulässiger Spannungszustand und ein damit verträglicher kinematisch zulässiger Bewegungszustand angeben, dann ist dieser Bewegungszustand instabil unter der Belastung $P$, und $P$ ist die exakte Traglast."

Ein kinematisch zulässiger Bewegungszustand heisst verträglich mit einem stabilen statisch zulässigen Spannungszustand, wenn Spannungen und Verzerrungsgeschwindigkeiten in jedem Punkt den Spannungs-Dehnungs-Beziehungen nach der Theorie des plastischen Potentials genügen. 
K A P I T E L I:

FLIESSBEDINGUNG UND FLIESSGESETZ FUER STAHLBETONSCHEIBEN

\section{FLIESSBEDINGUNGEN}

\subsection{Annahmen}

Es werden folgende Annahmen getroffen:

1. Die Annahmen der einfachen Plastizitätstheorie gemäss Abschnitt 1.2 sind zulässig.

2. Der Beton befindet sich in einem ebenen Druckspannungszustand. Seine Zugfestigkeit wird vernachlässigt.

3. Die Wirkung der Armierung wird als ebener einaxialer Spannungszustand in Stabrichtung erfasst ("verschmierte" Armierung).

4. Versagen der Armierung bei der ersten Rissebildung infolge zu geringem Armierungsgehalt, Versagen des Verbundes und lokales Versagen infolge Ausbrechen des Betons zwischen den Armierungsstäben werden ausgeschlossen.

Um zu einer einfachen mathematischen Formulierung zu gelangen, wird zudem angenommen:

5. Die Betonfliessfigur ist quadratisch (Fig. 2.1). Die Erhähung der zweiaxialen Betondruckfestigkeit gegenüber der einaxialen wird also vernachlässigt.

6. In Anbetracht der ungenauen Erfassung der zweiaxialen Betondruckfestigkeit, der starken Streuung der Betondruckfestigkeit und des meist geringen Armierungsgehaltes wird die Wirkung der Armierung auf Druck ebenfalls vernachlässigt.

7. Die Armierung ist als orthogonales Netz verlegt.

Annahme 1, insbesondere das notwendige duktile Bruchverhalten, ist für jene Fälle, wo die gesamte Armierung ins Fliessen kommt und der Beton auf Druck nicht massgebend wird, durch Versuche, Z.B. [35], genügend belegt. Unerwarteterweise ergibt aber Annahme 1 auch in Fällen massgebender Betondruckfestigkeit qualitativ vernünftige Resultate. Nichtorthogonale Armierung und der Einfluss einer wirklichkeitsnahen Erfassung der zweiaxialen Betondruckfestigkeit werden in Abschnitt 4 behandelt. Es zeigt sich, dass die Vereinfachung gemäss Annahme 5 die Fliessbedingungen der Stahlbetonscheibe in den für die Praxis wichtigen Bereichen, wo mindestens eine Armierungslage auf Zug ins Fliessen kommt, nicht beeinflusst.

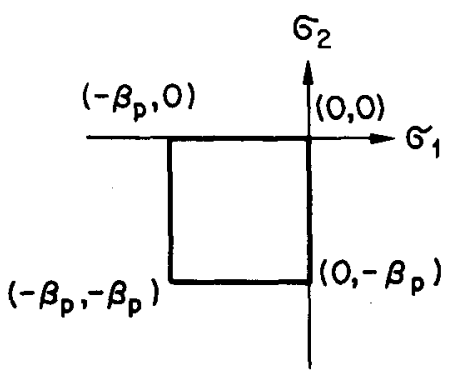

Fig. 2.1 Fliessbedingung für Beton 
Die auf das differentielle Scheibenelement der fig. 2.2 wirkenden Scheibenschnittkräfte $N_{x}, N_{y}, N_{x y}$
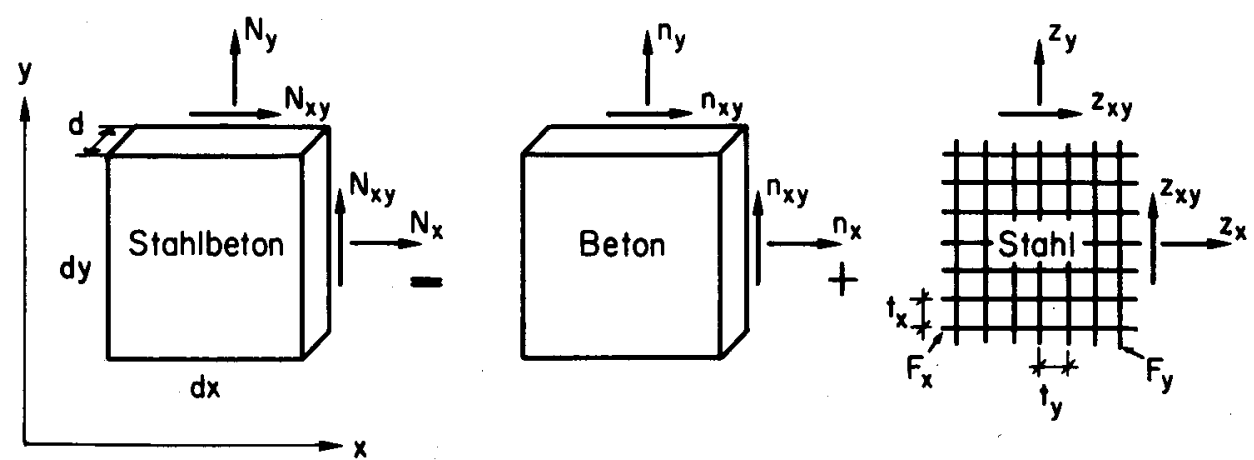

Fig. 2.2 Differentielles Scheibenelement

sind äquivalent der Summe der Schnittkräfte im Beton $n_{x}, n_{y}$, $n_{x y}$ und in der verschmiert gedachten Stahlarmierung $z_{x}, z_{y}, z_{x y}$. Das Koordinatensystem wird dabei mit Vorteil parallel zum orthogonalen Armierungsnetz eingeführt.

$\left.\begin{array}{l}N_{x}=n_{x}+z_{x}, \\ N_{y}=n_{y}+z_{y}, \\ N_{x y}=n_{x y}+z_{x y},\end{array}\right\}$

mit

$z_{x}=\frac{\sigma_{e} F_{x}}{t_{x}}, \quad z_{y}=\frac{\sigma_{e} F_{y}}{t_{y}}$.

Gemäss den Annahmen von Abschnitt 2.1 genügt der Beton den Fliessbedingungen

$-p_{b} \leq n_{1,2}=\frac{1}{2}\left(n_{x}+n_{y}\right) \pm \sqrt{\frac{1}{4}\left(n_{x}-n_{y}\right)^{2}+n_{x y}^{2}} \leq 0$,

oder gleichwertig

$n_{x y}^{2}=n_{x} n_{y}, \quad n_{x} \leq 0, n_{y} \leq 0$,

$n_{x y}^{2} \leq\left(P_{b}+n_{y}\right),\left(P_{b}+n_{x}\right) \geq 0,\left(P_{b}+n_{y}\right) \geq 0$,

während die Fliessbedingungen für den Stahl lauten

$\left.\begin{array}{rl}0 & \leq z_{x} \leq P_{x}, \\ 0 \leq z_{y} & \leq P_{y}, \\ z_{x y} & =0\end{array}\right\}$

Die plastischen Widerstände $P_{x}, P_{y}, P_{b}$ in (2.2) und (2.3) sind definiert durch $P_{x}=\frac{\sigma_{f} F_{x}}{t_{x}}, P_{y}=\frac{\sigma_{f} F_{y}}{t_{y}}, P_{b}=k \beta_{p} d$, 
wobei $\sigma_{f}$ die Fliess- oder Streckgrenze des Stahles, $F_{x} / t_{x}$ und $F_{y} / t_{y}$ die Querschnittsfläche der Armierung in $x$-bzw. $y$-Richtung pro Längeneinheit und $\beta_{p}$ die Betonprismenfestigkeit bezeichnen. $k \leq 1$ ist ein im konkreten Fall zu bestimmender Korrekturfaktor, mit dem berücksichtigt wird, dass die im Bruchzustand mitwirkende Scheibendicke und die massgebende Betanfestigkeit kleiner sein können als die nominelle Scheibendicke $d$ und die nominelle Prismenfestigkeit $\beta_{p}$.

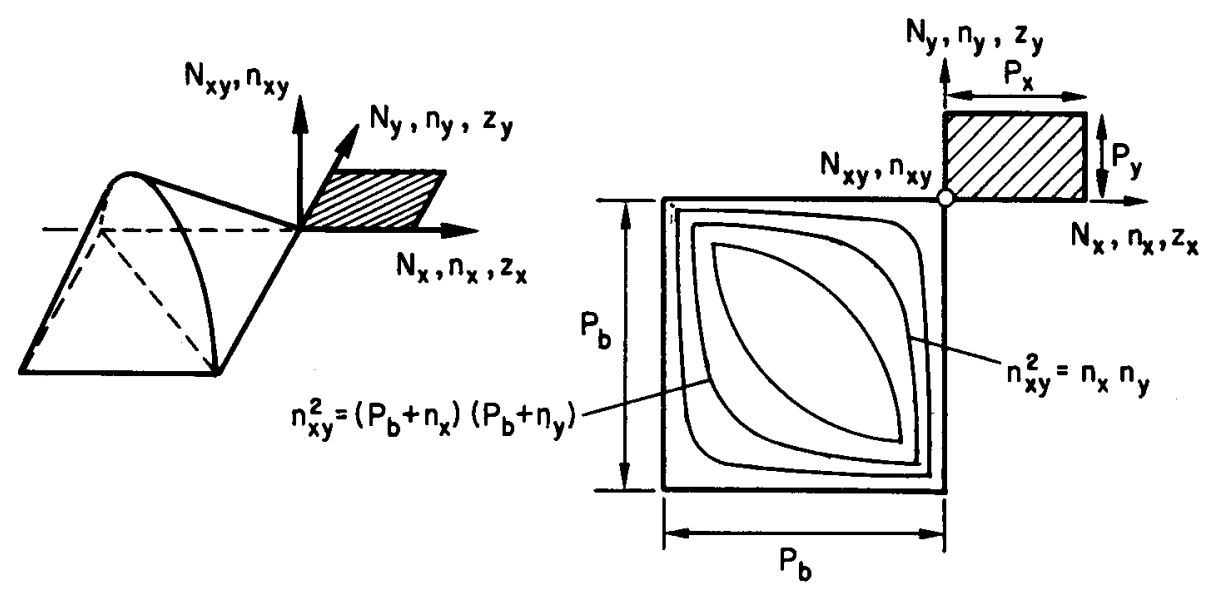

Fig. 2.3 Fliessfiguren für Beton und Stahl

Im dreidimensionalen Spannungsraum wird die Fliessfigur des Betons durch zwei elliptische Kegel dargestellt, während die Fliessfigur des Stahls ein Rechteck in der ( $N_{x}$, $N_{y}$ )-Ebene ist (Fig. 2.3). Die Menge der Spannungspunkte mit den Eigenschaften (2.2) bzw. (2.3) ist konvex. Die konvexe Menge der Spannungspunkte, die der gesuchten Fliessbedingung $\Phi\left(N_{x}, N_{y}, N_{x y}\right) \leq 0$ genügen, wird durch Gleichung (2.1) als Linearkombination der konvexen Mengen (2.2) und (2.3) definiert $[2,7]$.

Konvexe Mengen können auch durch ihre Stützfunktion beschrieben werden. Die Definition der Stützfunktion einer konvexen Menge $\Phi\left(N_{x}, N_{y}, N_{x y}\right) \leq 0$ lautet

$H\left(\dot{\varepsilon}_{x}, \dot{\varepsilon}_{y}, \dot{\gamma}_{x y}\right)=\operatorname{Max}\left(N_{x} \dot{\varepsilon}_{x}+N_{y} \dot{\varepsilon}_{y}+N_{x y} \dot{\gamma}_{x y}\right), \quad \Phi\left(N_{x}, N_{y}, N_{x y}\right) \leq 0$,

wobei $\dot{\varepsilon}_{x}, \dot{\varepsilon}_{y}, \dot{\gamma}_{x y}$ die $N_{x}, N_{y}, N_{x y}$ zugeordneten dualen Grössen sind [2, 7]. Wird die Gültigkeit der Theorie des plastischen Potentials vorausgesetzt (Abschnitt 3.1, 61. (3.1)), so beschreibt die Stützfunktion (2.4) einer kanvexen Fliessfigur $\Phi \leq 0$ die spezifische Dissipationsleistung infolge der plastischen Verzerrungsgeschwindigkeiten $\dot{\varepsilon}_{x}, \dot{\varepsilon}_{y}, \dot{\gamma}_{x y}$. Da der Satz gilt [2, 7], dass die Stützfunktion einer Linearkombination von konvexen Mengen gleich der Linearkombination ihrer Stützfunktionen ist, kann die Stützfunktion der gesuchten Fliessfigur für Stahlbetonscheiben sofort angeschrieben werden als Summe der spezifischen Dissipationsleistung von Beton und Stahl:

$H\left(\dot{\varepsilon}_{x}, \dot{\varepsilon}_{y}, \dot{\gamma}_{x y}\right)=\frac{1}{2} P_{x}\left(\left|\dot{\varepsilon}_{x}\right|+\dot{\varepsilon}_{x}\right)+\frac{1}{2} P_{y}\left(\left|\dot{\varepsilon}_{y}\right|+\dot{\varepsilon}_{y}\right)+\frac{1}{2} P_{b}\left(\left|\dot{\varepsilon}_{1}\right|-\dot{\varepsilon}_{1}+\left|\dot{\varepsilon}_{2}\right|-\dot{\varepsilon}_{2}\right)$,

mit $\dot{\varepsilon}_{1,2}=\frac{1}{2}\left(\dot{\varepsilon}_{x}+\dot{\varepsilon}_{y} \pm \sqrt{\left(\dot{\varepsilon}_{x}-\dot{\varepsilon}_{y}\right)^{2}+\dot{\gamma}_{x y}^{2}}\right)$.

Aus (2.5) ist auch ersichtlich, dass gleiche Verzerrungsgeschwindigkeiten in Beton und Stahl und somit die Erhaltung des Verbundes zwischen Beton und Stahl bis zum Kollaps vorausgesetzt werden. 
Für jeden Satz $\left(\dot{\varepsilon}_{x}, \dot{\varepsilon}_{y}, \dot{\gamma}_{x y}\right)$ folgt aus (2.4) eine Ungleichung

$N_{x} \dot{\varepsilon}_{x}+N_{y} \dot{\varepsilon}_{y}+N_{x y} \dot{\gamma}_{x y} \leq H\left(\dot{\varepsilon}_{x}, \dot{\varepsilon}_{y}, \dot{\gamma}_{x y}\right)$, für alle Sätze $\left(\dot{\varepsilon}_{x}, \dot{\varepsilon}_{y}, \dot{\gamma}_{x y}\right)$,

die unter der Voraussetzung erfüllt ist, dass $\Phi\left(N_{x}, N_{y}, N_{x y}\right) \leq 0$ gilt. Die Bedingungen, denen $N_{x}, N_{y}, N_{x y}$ genügen müssen, damit alle Ungleichungen (2.6) erfüllt sind, sind also die gesuchten Fliessbedingungen. Die Rechnung wird im Anhang A durchgeführt und ergibt folgende Fliessbedingungen:

$$
\begin{aligned}
& \text { : } \Phi_{1}=N_{x y y}^{2}-\left(P_{x}-N_{x}\right)\left(P_{y}-N_{y}\right) \leq 0 \text { für } \\
& N_{x} \leq P_{x}, \quad N_{y} \leq P_{y}, \quad N_{x}+N_{y} \geq P_{x}+P_{y}-P_{b} \\
& \text { I) : } \Phi_{2}=N_{x y}^{2}-\left(P_{b}+N_{x}\right)\left(P_{b}+N_{y}\right) \leq 0 \text { für } \\
& N_{x} \geq-P_{b}, N_{y} \geq-P_{b}, N_{x}+N_{y} \leq-P_{b} \\
& \text { (III) : } \Phi_{3}=N_{x y}^{2}-\left(\frac{1}{2} P_{b}\right) \leq 0 \text { für } \\
& -\frac{1}{2} P_{b} \leq N_{x} \leq P_{x}-\frac{1}{2} P_{b},-\frac{1}{2} P_{b} \leq N_{y} \leq P_{y}-\frac{1}{2} P_{b}
\end{aligned}
$$

(IV) : $\Phi_{4}=N_{x y}^{2}+\left(N_{x}-P_{x}+\frac{1}{2} P_{b}\right)^{2}-\left(\frac{1}{2} P_{b}\right)^{2} \leq 0$ für

$$
P_{x}-\frac{1}{2} P_{b} \leq N_{x} \leq P_{x}, \quad P_{x}-P_{b} \leq N_{x}+N_{y} \leq P_{x}+P_{y}-P_{b}
$$

$$
\begin{aligned}
: \Phi_{5}= & N_{x y}^{2}+\left(N_{y}-P_{y}+\frac{1}{2} P_{b}\right)^{2}-\left(\frac{1}{2} P_{b}\right)^{2} \leq 0 \text { für } \\
& P_{y}-\frac{1}{2} P_{b} \leq N_{y} \leq P_{y}, P_{y}-P_{b} \leq N_{x}+N_{y} \leq P_{x}+P_{y}-P_{b}
\end{aligned}
$$

(VI) $: \Phi_{G}=N_{x y}^{2}+\left(N_{x}+\frac{1}{2} P_{b}\right)^{2}-\left(\frac{1}{2} P_{b}\right)^{2} \leq 0$ für

$$
-P_{b} \leq N_{x} \leq-\frac{1}{2} P_{b}, \quad-P_{b} \leq N_{x}+N_{y} \leq P_{y}-P_{b}
$$

(VII) : $\Phi_{7}=N_{x y}^{2}+\left(N_{y}+\frac{1}{2} P_{b}\right)^{2}-\left(\frac{1}{2} P_{b}\right)^{2} \leq 0$ für

$$
-P_{b} \leq N_{y} \leq-\frac{1}{2} P_{b},-P_{b} \leq N_{x}+N_{y} \leq P_{x}-P_{b}
$$

$$
\begin{array}{rll}
\text { (VIII): } \Phi_{g}=N_{x}-P_{x} \leq 0 ; & (I X): \Phi_{g}=N_{y}-P_{y} \leq 0 \\
\Phi_{10}=-N_{x}-P_{b} \leq 0 ; & (X I): \Phi_{11}=-N_{y}-P_{b} \leq 0 .
\end{array}
$$

Diese Fliessbedingungen können aber auch leicht anschaulich verifiziert werden. Für feste Werte der Armierungsschnittkräfte $z_{x}$, $z_{y}$ innerhalb der schraffierten Stahlfliessfigur in Fig. 2.3 liegen die zulässigen Spannungspunkte $N_{x}, N_{y}$, $N_{x y}$ innerhalb und auf der translatorisch verschobenen Betonfliessfigur mit Zugkegelspitze im Punkt $\left(z_{x}, z_{y}\right)$. Also kann die gesuchte Fliessfigur als Umhüllende aller translatorisch verschobenen Betonfliessfiguren mit Zugkegelspitze innerhalb des schraffierten Rechtecks von Fig. 2.3 gewonnen werden.

Fig. 2.4 zeigt die Fliessfigur in axonometrischer, Fig. 2.5 in Höhenliniendarstellung. Dabei wird jeweils nur die obere Hälfte abgebildet, da die $\left(N_{x}, N_{y}\right)$-Ebene Symmetrieebene ist. Die Fliessfigur besteht aus zwei elliptischen Kegeln, nëmlich dem unverschobenen Druckfliesskegel (II) und dem mit seiner Spitze in den Punkt ( $P_{x}, P_{y}$ ) verschobenen Zugfliesskegel (I) der Betonfliessfigur. Diese zwei Kegel werden durch vier Kreiszylinderflächen IV, V, VI, VII 
und die Ebene III verbunden. Es ist sofort ersichtlich, dass die fliessfigur nicht invariant gegenüber Drehungen des Koordinatensystems, d.h. anisotrop ist: Isotropie setzt voraus, dass alle Schnitte der Fliessfigur mit Ebenen $N_{x}+N_{y}=$ konst. Abbildungen des Mohr'schen Spannungskreises, d.h. Ellipsen sind, deren Zentrum auf der Achse $N_{x}-N_{y}=N_{x y}=0$ liegt, und deren grösserer, in der Ebene $N_{x y}=0$ liegender Hauptabschnitt um einen Faktor $\sqrt{2}$ grösser ist als der kleinere.

Der Einfluss der Berücksichtigung von Druckkräften in der Armierung ist auch sofort ersichtlich. Der Betondruckfliesskegel wird nun ebenfalls verschoben, seine Spitze liegt nun im Punkt $\left(-P_{x},-P_{y}\right)$, und die Verbindungsflächen der Kegel werden in ihrer Ausdehnung verdoppelt. In dieser form ist die Fliessfigur nicht neu [15].

In beiden bisher zur Berechnung des Bruchwiderstandes von Stahlbetonträgern unter Torsion und Biegung vorgeschlagenen Kollapsmechanismen [17, 18] wird das dem angenommenen Fliessregime zugeordnete Fliessgesetz verletzt. In den nächsten Abschnitten folgen deshalb eine kurze Darstellung der Statik und eine etwas ausführlichere der Kinematik der einzelnen Fliessregimes.

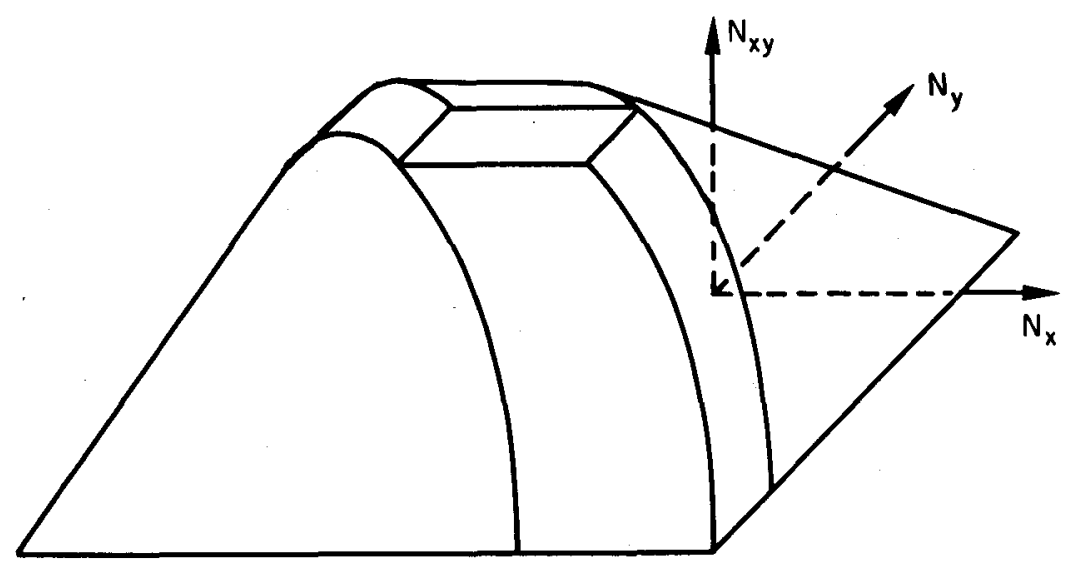

Fig. 2.4 Axonometrische Darstellung der Fliessfigur (2.7)

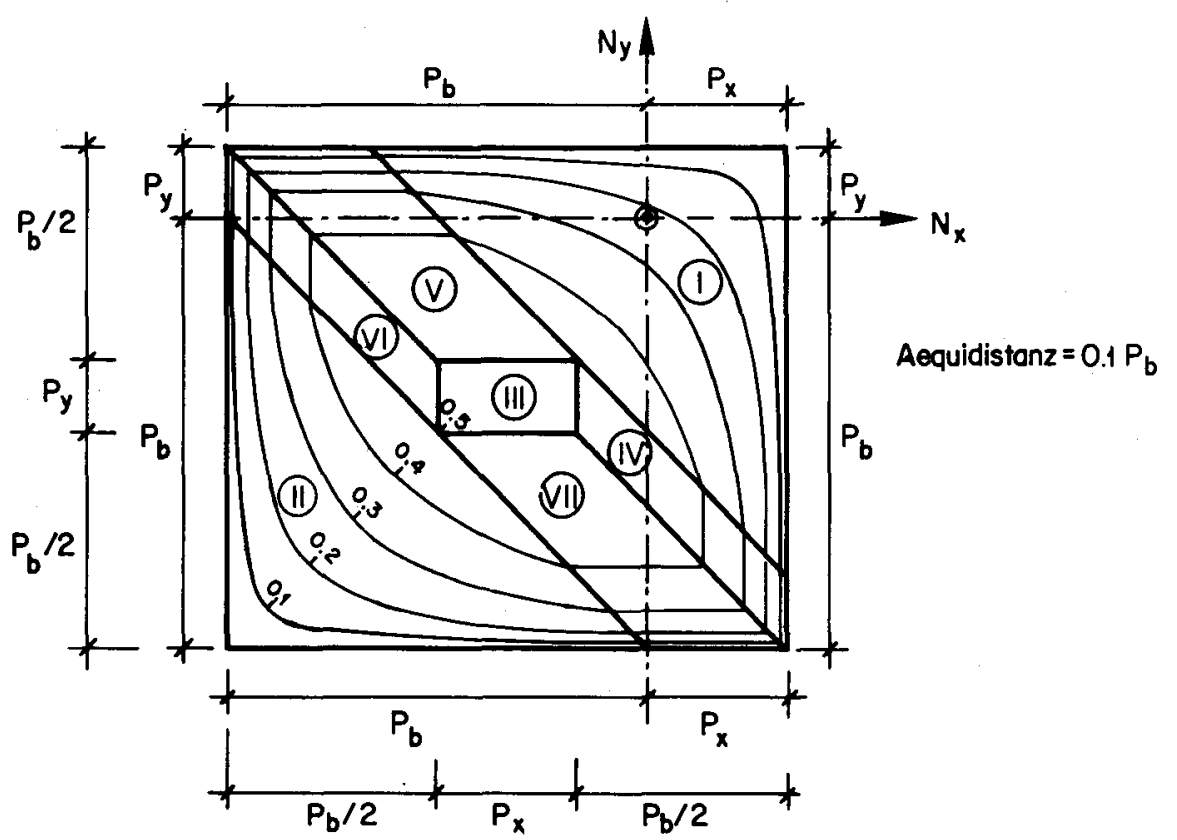

Fig. 2.5 Höhenliniendarstellung der Fliessfigur ( 2.7) 
Welche Fliessbedingungen der einzelnen Komponenten Beton und Stahl auf den verschiedenen Fliessflächen massgebend werden, ist rein geometrisch aus Figuren 2.3, 2.4 und 2.5 ersichtlich. Die Resultate sind in Fig. 2.6 in einer schematischen Aufsicht der Fliessfigur zusammengefasst. Dabei bezeichnet $n_{1}$ die grössere, $n_{2}$ die kleinere Betonhauptspannung.

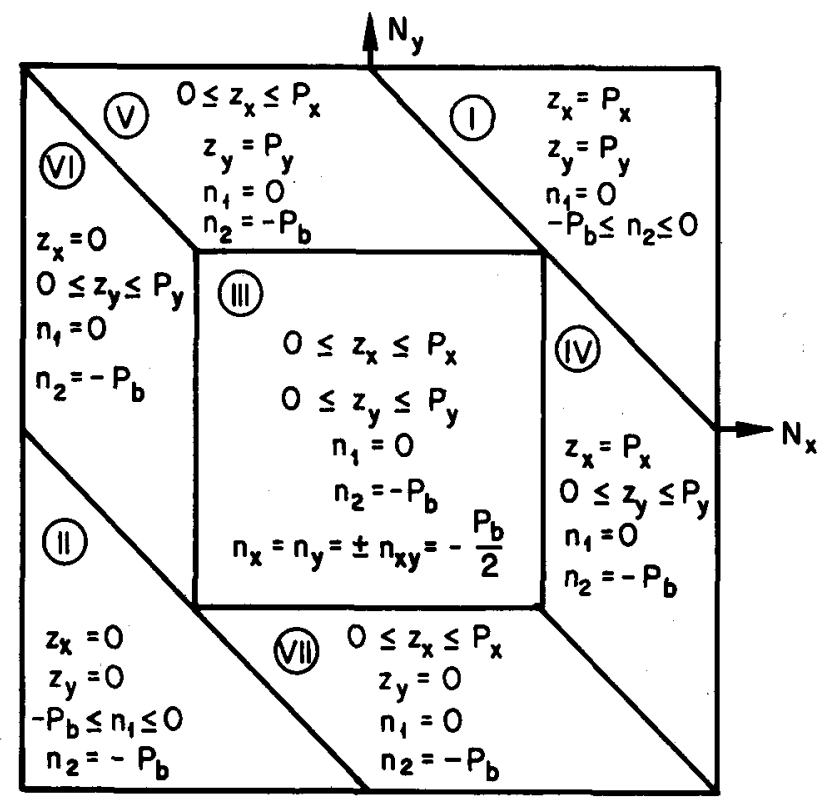

Fig. 2.6 Mossgebende Spannungszustände in Beton und Stahl

Bezüglich der Spannungszustände im Beton können die verschiedenen Fliessflächen folgendermassen charakterisiert werden:

- Auf der Fliessfläche I wird nur die null gesetzte Betonzugfestigkeit, auf der Fliessfläche II nur die Betondruckfestigkeit massgebend, während für die Fliessflächen III bis VII beide massgebend werden.

- Auf allen Fliessflächen ausser II befindet sich somit der Beton mit $n_{1}=0$ in einem einaxialen Druckspannungszustand. Dieser entspricht dem im Fachwerkmodel 1 angesetzten Druckfeld.

- Auf den Fliessflächen IV bis VII ist der Betonspannungszustand mit $n_{1}=0, n_{2}=-P_{b}$ bis auf die Hauptspannungsrichtung, auf Fliessfläche III sogar vollständig festgelegt.

Fig. 2.7 veranschaulicht die Spannungszustände im Beton noch anhand Mohr'scher Kreise.

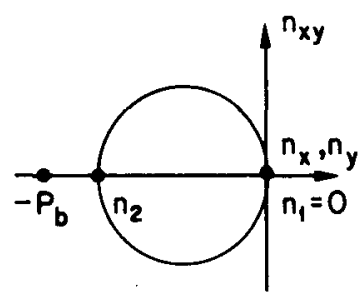

Fliessfläche !

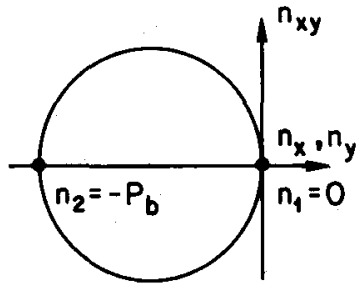

Fliessfldchen $\|I-V\|$

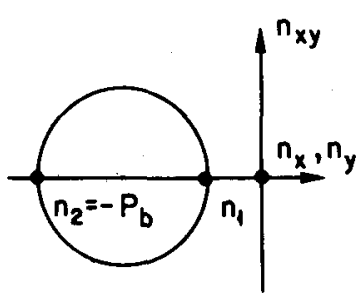

Fliessflöche \|

Fig. 2.7 Spannungszustände im Beton 
Die massgebenden Fliessgrenzen der Armierung werden unabhängig von ihrem numerischen Wert angenommen. Die gemäss Fig. 2.3 null gesetzte Druckfliessgrenze der Armierung wird deshalb immer noch als solche bezeichnet, und die folgenden Aussagen gelten sinngemäss auch, wenn Druckspannungen im Stahl berücksichtigt werden.

In bezug auf die Spannungszustände im Stahl können die Fliessflächen folgendermassen charakterisiert werden:

- Auf der Fliessfläche I bzw. II liegen die Spannungen beider Armierungen an der Zug- bzw. Druckfliessgrenze, während an der Fliessfläche III keine der Stahlfliessgrenzen massgebend wird.

- Die Fliessflächen IV bis VII dagegen sind dadurch ausgezeichnet, dass die Spannung jeweils einer Armierung an der Zug- oder Druckfliessgrenze liegt, während sie für die andere beliebige Werte dazwischen annehmen kann.

- Dabei ist an den Fliessflächen IV und V die Zug-, an VI und VII die Druckfliessgrenze massgebend.

Zusammenfassend und im Hinblick auf die in Abschnitt 3 behandelte Kinematik der Fliessregimes werden die Fliessflächen noch durch die nicht massgebenden Fliessgrenzen der einzelnen Komponenten Beton und Stahl charakterisiert: Für Fliessfläche I wird die Betondruckfestigkeit, für II die Betonzugfestigkeit nicht massgebend, während für III die Fliessgrenzen beider, für IV bis VII die Fliessgrenzen je einer Armierung nicht massgebend werden. 


\section{FLIESSGESETZ UND KINEMATIK}

Für die Berechnung der Traglast brauchen nur die Spannungs- und Bewegungszustände beim Erreichen der Traglast untersucht zu werden. Die Traglast ist diejenige Last, unter der zum ersten Mal ohne Lastzuwachs zusätzliche Verformungen auftreten. Da folglich die Spannungsinkremente und somit auch die elastischen Verzerrungsinkremente verschwinden, wird der Bewegungszustand beim Erreichen der Traglast durch die plastischen Verzerrungsinkremente bzw. Verzerrungsgeschwindigkeiten allein beschrieben. Im folgenden sind deshalb mit Verzerrungsgeschwindigkeiten immer die plastischen Verzerrungsgeschwindigkeiten während des Kollapses gemeint.

\subsection{Spannungs-Verzerrungs-Beziehungen}

Aufgrund der Theorie des plastischen Potentials ist das Fliessgesetz, das die plastischen Verzerrungsgeschwindigkeiten und die Spannungen verknüpft, für $n$ Fliessbedingungen der Form $\Phi_{k}\left(\sigma_{i j}\right) \leq 0$ gegeben durch

$\left.\begin{array}{l}\dot{\varepsilon}_{i j}=\sum_{k=1}^{n} \frac{\partial \Phi_{k}\left(\sigma_{i j}\right)}{\partial \sigma_{i j}} \dot{\lambda}_{k}, \quad i, j=x, y, z \\ \dot{\lambda}_{k}=0 \text { für } \Phi_{k}<0, \dot{\lambda}_{k} \geq 0 \text { für } \Phi_{k}=0,\end{array}\right\}$

wobei für $\Phi_{k}$ bei Stahlbetonscheiben die Ausdrücke (2.7) einzusetzen sind.

Für das Fliessregime I $\left(\Phi_{1}=0, \Phi_{k \neq 1}<0\right)$ ergeben sich die Verzerrungsgeschwindigkeiten zu $\dot{\varepsilon}_{x}=\frac{\partial \Phi_{1}}{\partial N_{x}} \dot{\lambda}=\left(P_{y}-N_{y}\right) \dot{\lambda}=-n_{y} \dot{\lambda} \geq 0$,

$\dot{\varepsilon}_{y}=\frac{\partial \Phi_{1}}{\partial N_{y}} \dot{\lambda}=\left(P_{x}-N_{x}\right) \dot{\lambda}=-n_{x} \dot{\lambda} \geq 0$,

$\dot{\gamma}_{x y}=\frac{\partial \Phi_{1}}{\partial N_{x y}} \dot{\lambda}=2 N_{x y} \dot{\lambda} \quad=2 n_{x y} \dot{\lambda}$.

Dabei wurde schon von den Beziehungen in Fig. 2.6, von (2.1), (2.2), (2.3) und von den Gü1tigkeitsgrenzen in (2.7) Gebrauch gemacht. Für die Richtung der kleineren Hauptdehnungsgeschwindigkeit folgt

$\tan 2 \alpha=\frac{-\dot{\gamma}_{x y}}{\dot{\varepsilon}_{y}-\dot{\varepsilon}_{x}}=\frac{-2 n_{x y}}{n_{y}-n_{x}}$,

wobei die Definition des Winkels $\alpha$ aus Fig. 3.1 ersichtlich ist.

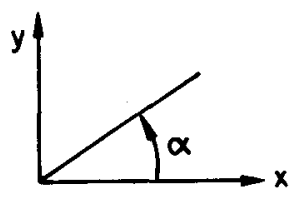

Fig. 3.1 Definition von $\alpha$ 
Entsprechend ergibt sich für das Fliessregime IV $\left(\Phi_{4}=0, \Phi_{k \neq 4}<0\right)$

$\dot{\varepsilon}_{x}=2\left(N_{x}-P_{x}+\frac{1}{2} P_{b}\right) \dot{\lambda}=\left(2 n_{x}+P_{b}\right) \dot{\lambda} \geq 0$,

$\dot{\varepsilon}_{y}=0, \dot{\gamma}_{x y}=2 N_{x y} \dot{\lambda}=2 n_{x y} \dot{\lambda}$

\}

$\tan 2 a=\frac{-\gamma_{x y}}{\dot{\varepsilon}_{y}-\dot{\varepsilon}_{x}}=\frac{-2 n_{x y}}{-2 n_{x}-P_{b}}=\frac{-2 n_{x y}}{n_{y}-n_{x}}$,

und für Fliessregime VII $\left(\Phi_{7}=0, \Phi_{k \neq 7}<0\right)$

$$
\begin{aligned}
& \dot{\varepsilon}_{x}=0, \quad \dot{\gamma}_{x y}=2 N_{x y} \dot{\lambda}=2 n_{x y} \dot{\lambda}, \\
& \dot{\varepsilon}_{y}=2\left(N_{y}+\frac{1}{2} P_{b}\right) \dot{\lambda}=\left(2 n_{y}+P_{b}\right) \dot{\lambda} \leq 0, \\
& \tan 2 \alpha=\frac{-\dot{\gamma}_{x y}}{\dot{\varepsilon}_{y}-\dot{\varepsilon}_{x}}=\frac{-2 n_{x y}}{2 n_{y}+P_{b}}=\frac{-2 n_{x y}}{n_{y}-n_{x}},
\end{aligned}
$$

wenn noch zusätzlich beachtet wird, dass für die Fliessregimes IV und VII gemäss Fig. 2.6 aus $n_{1}=0, n_{2}=-P_{b}, n_{1}+n_{2}=n_{x}+n_{y}=-P_{b}$ folgt.

Infolge der Anisotropie sind im allgemeinen die Hauptachsen der Scheibenspannungen $N_{x}$, $N_{y}$, $N_{x y}$ und der Verzerrungsgeschwindigkeiten nicht identisch. Dagegen bezeichnet aber der letzte Ausdruck in den Gleichungen (3.3), (3.5), (3.7) die Richtung der kleineren Betonhauptspannungen bzw. die Betondrucktrajektorienrichtung. Wie leicht auch für die übrigen Fliessregimes verifiziert werden kann,fallen also die Hauptachsen der plastischen Verzerrungsgeschwindigkeiten immer mit den Hauptachsen des Spannungszustandes im Beton zusammen.

\subsection{Kinematik der einzelnen Fliessregimes}

Ausgehend von den Spannungs-Verzerrungs-Beziehungen des. Abschnittes 3.1 sollen nun die kinematischen Bindungen zusammengestellt werden, denen die Verzerrungsgeschwindigkeiten in den einzelnen Fliessregimes genügen müssen (Fig. 3.2).

Als kinematische Bindung wird derjenige Teil der Verträglichkeitsbeziehungen (3.1) zwischen Spannungs- und Bewegungszustand bezeichnet, der sich in den Verzerrungsgeschwindigkeiten allein ausdrücken lässt und allgemein für ein gesamtes Fliessregime gilt. Ein Bewegungszustand ist mit einem speziellen Spannungszustand in einem Fliessregime verträglich, wenn er die kinematischen Bindungen des Fliessregimes erfüllt, und wenn - wie in Abschnitt 3.1 gezeigt die Hauptachsen der Verzerrungsgeschwindigkeiten und des Betonspannungszustandes zusammenfallen.

Für Fliessregime $I\left(\Phi_{1}=0, \Phi_{k \neq 1}<0\right)$ folgt aus (3.2) und durch Einsetzen von (3.2) in die Fliessbedingung $\Phi_{1}=0$

$\left(\frac{1}{2} \dot{\gamma}_{x y}\right)^{2}=\dot{\varepsilon}_{x} \dot{\varepsilon}_{y}, \dot{\varepsilon}_{x} \geq 0, \dot{\varepsilon}_{y} \geq 0$

oder, wenn $\dot{\varepsilon}_{1}$ die grössere, $\dot{\varepsilon}_{2}$ die k.leinere Hauptdehnungsgeschwindigkeit bezeichnet,

$\dot{\varepsilon}_{1} \geq \dot{\varepsilon}_{2}=0$. 
Aus (3.4) liest man für Fliessregime IV ab

$\dot{\varepsilon}_{x} \geq 0, \quad \dot{\varepsilon}_{y}=0$,

und aus (3.6) für Fliessregime VII

$\dot{\varepsilon}_{x}=0, \quad \dot{\varepsilon}_{y} \leq 0$

Für die einer Kante oder Spitze der Fliessfigur entsprechenden Fliessregimes erhält man die verträglichen Bewegungszustände gemäss Fliessgesetz (3.1) als Linearkombinationen der Bewegungszustände in den angrenzenden Fliessregimes. So ergeben die Linearkombinationen mit positiven Koeffizienten der Bewegungszustände (3.9) und (3.10) die mit dem Fliessregime GH $\left(\Phi_{4}=\Phi_{7}=0\right)$ verträglichen Bewegungszustände

$\dot{\varepsilon}_{x} \geq 0, \quad \dot{\varepsilon}_{y} \leq 0$

Für das der Spitze des Zugfliesskegels entsprechende Fliessregime $A\left(\Phi_{1}=\Phi_{8}=\Phi_{9}=0\right)$ ergibt die Linearkombination von (3.8) mit den auf den Fliessebenen $\Phi_{B}=0$ bzw. $\Phi_{g}=0$ senkrecht stehenden Verzerrungsgeschwindigkeitsvektoren

$\left(\frac{1}{2} \dot{\gamma}_{x y}\right)^{2} \leq \dot{\varepsilon}_{x} \dot{\varepsilon}_{y}, \quad \dot{\varepsilon}_{x} \geq 0, \quad \dot{\varepsilon}_{y} \geq 0$,

oder $\dot{\varepsilon}_{1} \geq \dot{\varepsilon}_{2} \geq 0$.

Die Ergebnisse sind zusammen mit den leicht verifizierbaren Resultaten für die übrigen Fliessregimes in Fig. 3.2 in einer schematischen Aufsicht der Fliessfigur zusammengestellt.

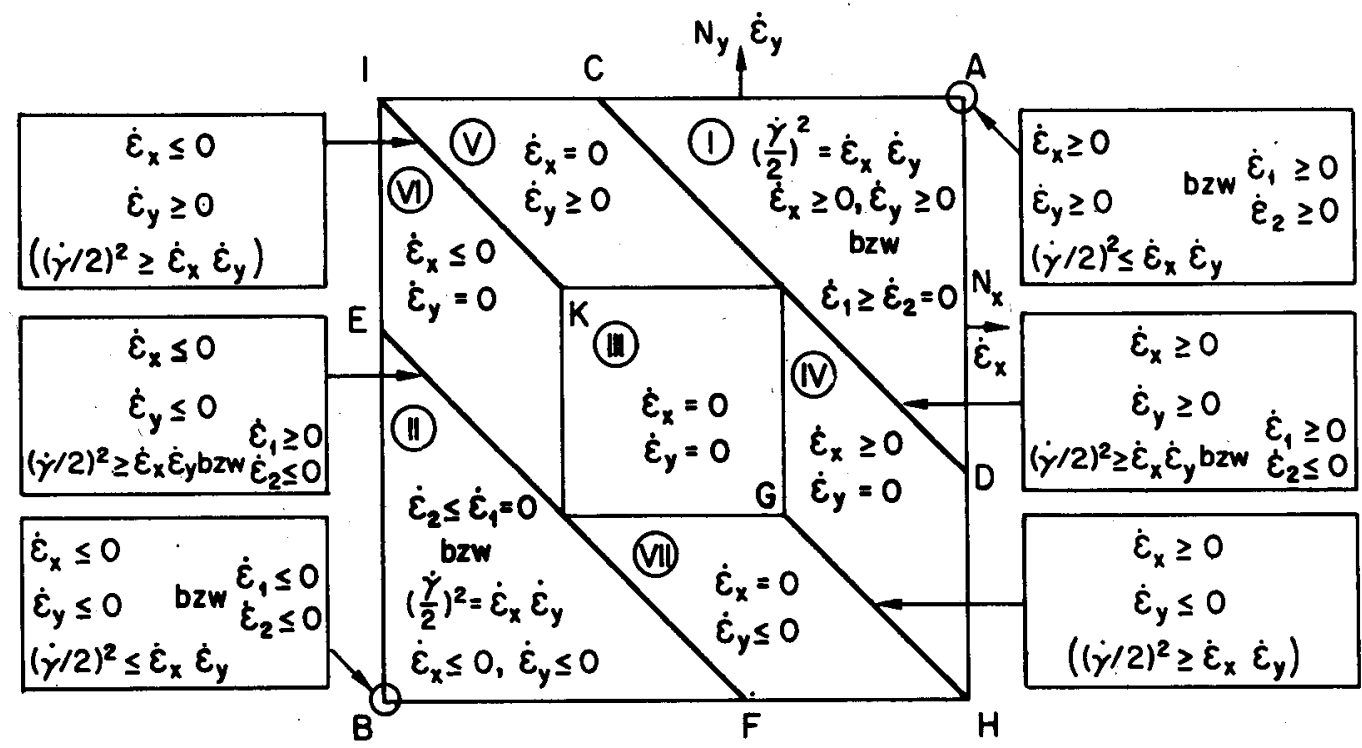

Fig. 3.2 Kinematische Bindungen in den einzelnen Fliessregimes

In den Fliessregimes I und $A$ bzw. II und $B$ sind also nur Geschwindigkeitsfelder zulässig, die keine Stauchungen bzw. Dehnungen aufweisen. In den übrigen Fliessregimes dagegen ist der Bewegungszustand lediglich durch die starr bleibenden bzw. auf Zug oder Druck fliessenden Armierungsstäbe beschränkt. Fig. 3:3 veranschaulicht die Verhältnisse noch anhand Mohr'scher Kreise. 


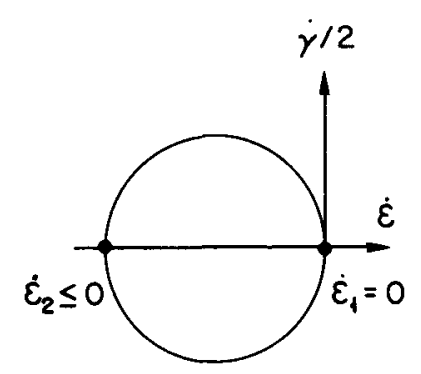

Regime $\|$

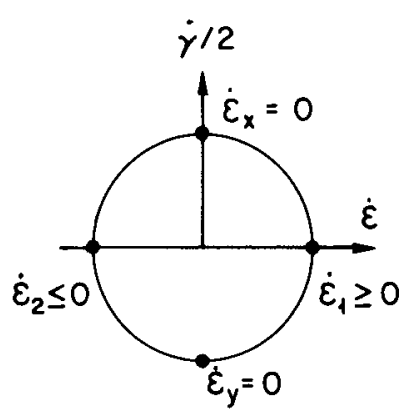

Regime III

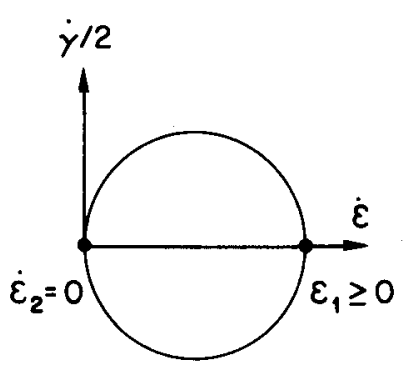

Regime 1

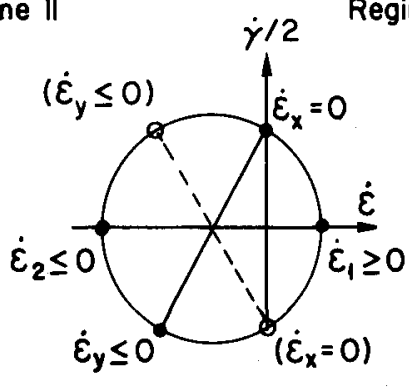

Regime VII

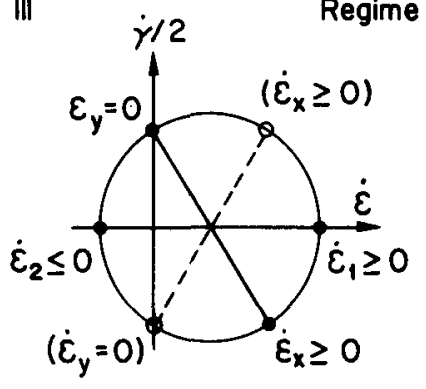

Regime IV

Fig. 3.3 Verträgliche Bewegungszustände für verschiedene Fliessregimes

Die kinematischen Bindungen der Fig. 3.2 stellen lediglich die duale, kinematische Interpretation der in Fig. 2.6 zusammengestellten, massgebenden und nicht massgebenden Fliessgrenzen der Komponenten Beton und Stahl dar. Insbesondere sind also (3.8) und (3.12) lediglich die kinematische Konsequenz des Sachverhaltes, dass im Bereich der fliessregimes I und A die Betondruckfestigkeit nicht massgebend wird. Obschon die Beziehungen von Fig. 3.2 selbstverständlich erscheinen mögen, wurde (3.8) bei allen bisher vorgeschlagenen Kollapsmechanismen für unterarmierte Stahlbetonbalken unter Torsion und Biegung übersehen. Dies dürfte vor allem darauf zurückzuführen sein, dass die Fliessbedingung $\Phi_{1} \leq 0$ nur implizit verwendet wurde, und dass die im Kollapszustand betrachteten "Risse" nicht als Unstetigkeitslinien des Geschwindigkeitsfeldes im Sinne der ebenen Plastizitätstheorie aufgefasst wurden. Das Thema des nächsten Abschnittes bilden deshalb die Unstetigkeitslinien des Geschwindigkeitsfeldes. 


\subsection{Unstetige Geschwindigkeitsfelder}

Aus der Plastizitätstheorie ist bekannt, dass die Geschwindigkeitsfelder der Kollapsmechanismen oft unstetig sind. Im folgenden wird deshalb untersucht, welche Sprünge des Geschwindigkeitsfeldes mit den verschiedenen Fliessregimes verträglich sind. Dabei muss eine Unstetigkeitslinie als Grenzfall einer endlichen Uebergangszone aufgefasst werden mit wohl stark aber stetig (z.B. linear) sich veränderndem Geschwindigkeitsfeld (Fig. 3.4).

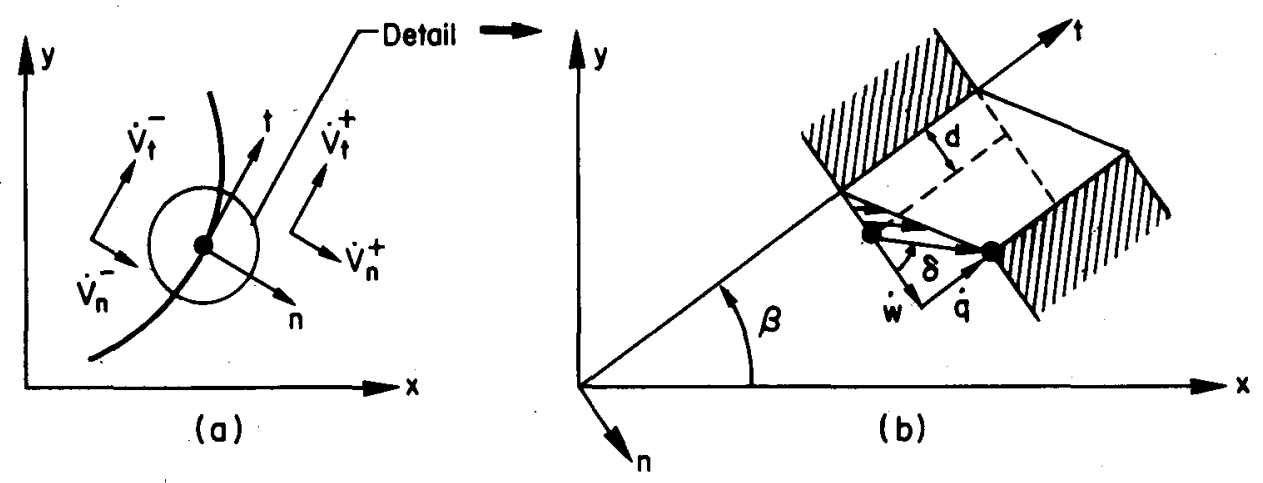

Fig. 3.4 Unstetigkeitslinie

Die Verzerrungsgeschwindigkeiten in der Vebergangszone ergeben sich für gegen 0 strebende Dicke d zu

$\left.\begin{array}{l}\dot{\varepsilon}_{n}=\frac{\dot{w}}{d}, \quad \dot{\gamma}_{n t}=\frac{\dot{q}}{d}, \quad \dot{\varepsilon}_{t} / \dot{\varepsilon}_{n} \text { bzw. } \dot{\varepsilon}_{t} / \dot{\gamma}_{n t} \rightarrow 0, \\ \dot{\varepsilon}_{1}=\frac{1}{2 d}\left(\dot{w}+\sqrt{\dot{w}^{2}+\dot{q}^{2}}\right), \dot{\varepsilon}_{2}=\frac{1}{2 d}\left(\dot{w}-\sqrt{\dot{w}^{2}+\dot{q}^{2}}\right),\end{array}\right\}$

wenn $\dot{w}$ bzw. $\dot{q}$ den Sprung in der zur Unstetigkeitslinie normalen bzw. tangentialen Geschwindigkeitskomponente bezeichnet:

$\dot{w}=\dot{v}_{n}^{+}-\dot{v}_{n}^{-}, \quad \dot{q}=\dot{v}_{t}^{+}-\dot{v}_{t}^{-}$.

Die kinematische Bindung (3.8) für Fliessregime I bzw. (3.12) für Fliessregime A kann offensichtlich nur mit

$\dot{q}=0, \quad \dot{w} \geq 0$,

erfüllt werden. Nur die Normalkomponente der Geschwindigkeit darf unstetig verlaufen, die Unstetigkeitslinie ist Hauptrichtung der Verzerrungsgeschwindigkeiten und fällt gemäss Abschnitt 3.1 für Regime I mit der Betondrucktrajektorie zusammen: $\alpha=\beta$.

Entsprechend folgt aus den kinematischen Bindungen für die Fliessregimes II und $B \dot{q}=0$, $\dot{w} \leq 0$. Da jedoch in Bereichen massgebender fliessbedingung II mit der exakten Fliessfigur gemäss Abschnitt 4.2 gearbeitet werden sollte, ist, dieser fall nicht weiter interessant.

Verläuft dagegen an einer Unstetigkeitslinie auch.die Tangentialgeschwindigkeit unstetig,

$\dot{q} \neq 0, \quad \dot{w} \geq 0$,

$\dot{q} \neq 0, \quad \dot{w} \leq 0$, 
so wird mit (3.13) $\dot{\varepsilon}_{1} \geq 0, \dot{\varepsilon}_{2} \leq 0$, und $\dot{\varepsilon}_{1}+\dot{\varepsilon}_{2}=\dot{\varepsilon}_{x}+\dot{\varepsilon}_{y}=\dot{w} / d \geq 0$ im Fall (3.16a) bzw. $\dot{\varepsilon}_{1}+\dot{\varepsilon}_{2} \leq 0$ im Fall (3.16b). Je nach Dehnungsgeschwindigkeit in $x$ - und y-Richtung ist somit (3.16a) mit einem der Fliessregimes III, IV, V, C-D, G-H oder I-K verträglich, während (3.16b) einem der Regimes III, VI, VII, E-F, G-H oder I-K zuzuordnen ist (Fig. 3.2).

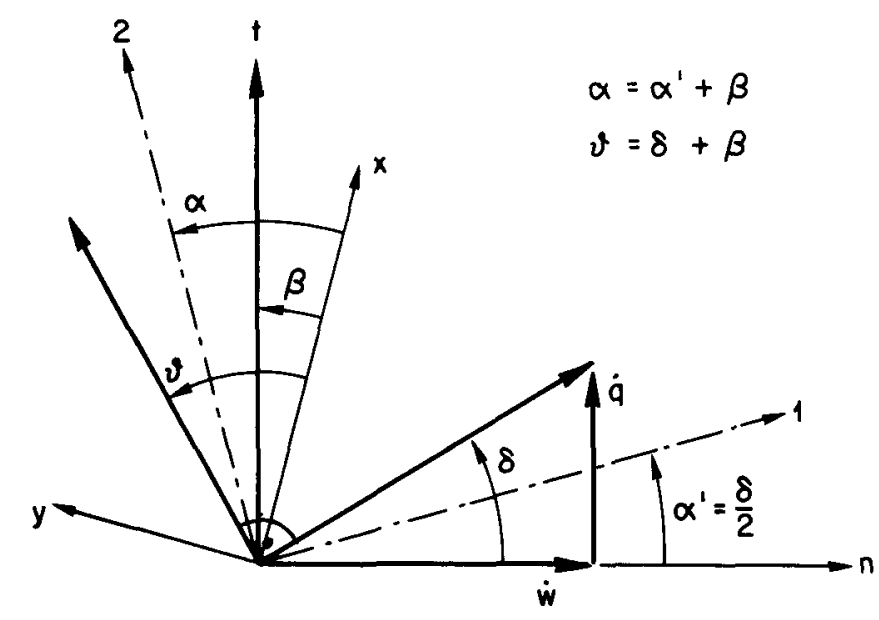

Fig. 3.5 Lage des Houptochsensystems

Für die Richtung der grösseren Hauptdehnungsgeschwindigkeit $\dot{\varepsilon}_{1} \geq 0$ folgt (Figuren 3.4 und 3.5):

$$
\begin{aligned}
\tan 2 \alpha^{\prime}=\frac{\dot{\gamma}_{n t}}{\dot{\varepsilon}_{n}-\dot{\varepsilon}_{t}} & =\frac{\dot{q}}{\dot{w}}=\tan \delta, \\
\alpha^{\prime} & =\frac{\delta}{2} .
\end{aligned}
$$

Die Hauptachse 1 halbiert den Winkel zwischen der Normalen zur Unstetigkeitslinie und der Sprungrichtung. Die Richtung der parallel zur Hauptachse 2 verlaufenden Betondrucktrajektorien halbiert somit für $\dot{w} \geq 0$ den kleineren, für $\dot{w} \leq 0$ den grösseren Winkel zwischen der Tangente an die Unstetigkeitslinie und der Senkrechten zur Sprungrichtung. Ihre Neigung bezüglich der x-Achse ergibt sich aus Fig. $3.5 \mathrm{zu}$

$\alpha=\beta+\frac{\delta}{2}=\frac{1}{2}(\beta+\vartheta)$

Die symmetrische Form (3.18) kann unmittelbar anhand eines Mohr'schen Kreises verifiziert werden, wenn beachtet wird, dass $\beta$ und $\vartheta$ die zwei Richtungen verschwindender Dehnungsgeschwin- . digkeit bezeichnen.

Für die an einer Unstetigkeitslinie entstehende Dissipationsleistung folgt aus den Gleichungen (2.5) und (3.13), dass diese nur für Unstetigkeiten (3.15) mit $\dot{\varepsilon}_{1} \geq \dot{\varepsilon}_{2}=0$ durch die Dissipationsleistung der Armierung allein gegeben ist, während in allen anderen Fällen der Beton in der Uebergangszone gestaucht wird und auch einen Beitrag leistet. Insbesondere bei Unstetigkeiten ( $3.16 a)$ darf die Dissipationsleistung des Betons nicht vergessen werden. Dies ist noch in Fig. 3.6 veranschaulicht.

Der Beton befindet sich in den (3.16) zugeordneten Fliessregimes in einem einaxialen, zur Hauptachse 2 parallelen Spannungszustand an der Druckfliessgrenze. Der Betonspannungsvektor in einem Schnitt entlang der Unstetigkeitslinie ist also weder null noch steht er senkrecht 


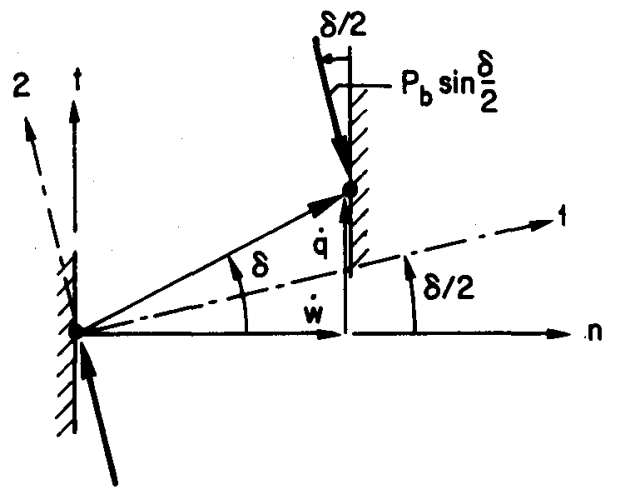

Fig. 3.6 Betonsponnungen entlang einer Unstetigkeitslinie (3.16a)

zur Sprungrichtung.

Wird dagegen im Fall (3.16a) für beliebige Neigung der Unstetigkeitslinie nur die Dissipationsleistung der Armierung berücksichtigt, so ist dies nach der Theorie des plastischen Potentials gleichbedeutend mit der Annahme der unrealistischen Betonfliessfigur von Fig. 3.7. Diese Fliessbedingung ist aus der Bodenmechanik bekannt und gehört zu einem kohäsionslosen Coulomb-Material im ebenen Dehnungszustand. Der Winkel der inneren Reibung $\Phi=90-\delta$ wird dabei sogar variabel angenommen, wenn die Sprungrichtung $\delta$ Funktion derjenigen Parameter des Mechanismus ist, bezüglich welchen der Traglastfaktor minimalisiert wird.

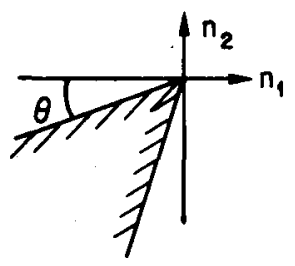

$$
\begin{aligned}
& \text { - } \Phi=90-8 \\
& \text { - } \tan \theta=\tan ^{2} \frac{\delta}{2}=\tan ^{2}\left(45-\frac{\Phi}{2}\right)
\end{aligned}
$$

Fig. 3.7 Fliessbedingung von Coulomb (ebener Dehnungszustand)

Dieser Fehler kann leicht unterlaufen, wenn Mechanismen ohne explizite Fliessbedingung direkt von dem im Versuch beobachteten Rissebild und Risseverhalten abgeleitet werden. Ein solcher Mechanismus ist der im Abschnitt 8.1 behandelte "Skew-Bending"-Mechanismus für Stahlbetonbalken unter Torsion und Biegung. Wirkliche Risse und theoretische "Risse" eines Kollapsmechanismus sind jedoch auseinanderzuhalten. Letztere sind im Rahmen des Traglastverfahrens als Unstetigkeiten in den Verschiebungsinkrementen beim Erreichen der Traglast aufzufassen. Wird angenommen, dass entlang eines "Risses" beide Armierungen fliessen und die Betonzugfestigkeit null ist, so muss sich die Scheibe im Fliessregime I oder A befinden, und die inkrementale "Risse"-Deffnung kann gemäss (3.15) nur senkrecht zur "Riss"-Richtung erfolgen.

Es stellt sich nun noch die Frage nach der Beziehung zwischen den Unstetigkeiten (3.15) bzw. (3.16a) und den wirklichen Rissen der Stahlbetonscheibe wie sie im Versuch vor der Ausbildung des Bruchmechanismus beobachtet werden können. Am ehesten lassen sich die Rissrichtungen mit den Richtungen der Betondrucktrajektorien in Beziehung bringen (Abschnitte 5 und 7). Sie folgen - mit durch die partiell immer vorhandenen Betonzugspannungen bedingter Verzögerung - der theoretischen, vom homogenen übër den gerissenen zum Kollaps-Zustand sich ändernden Richtung der Betondrucktrajektorien [16, 36, 40]. Damit liegt es nahe, die zweite Hauptdehnungsrichtung bzw. die Richtung der Betondrucktrajektorien als theoretische Rissrichtung und die Unstetigkeiten (3.15) als die theoretischen Risse der idealisierten Stahlbetonscheibe ohne 
Betonzugfestigkeit zu interpretieren. Solche Unstetigkeiten sallen deshalb "Kollapsrisse" genannt werden. "Kollapsrisse" treten also entlang den Betondrucktrajektorien auf und können sich während des kollapses nur senkrecht öffnen. In dieser für den Praktiker anschaulichen Form wurde (3.15) in [22] übernommen. Dagegen können Unstetigkeiten (3.16a) bis zu $45^{\circ}$ geneigt zu den Betondrucktrajektorien und den beobachteten Rissen auftreten (Abschnitt 7). Sie ebenfalls als Risse zu bezeichnen wäre deshalb verwirrend und sie sollen im folgenden zusammen mit den Unstetigkeiten (3.16b) "Gleitlinien" genannt werden. Die Resultate dieses Abschnittes sind in Fig. 3.8 zusammengefasst.

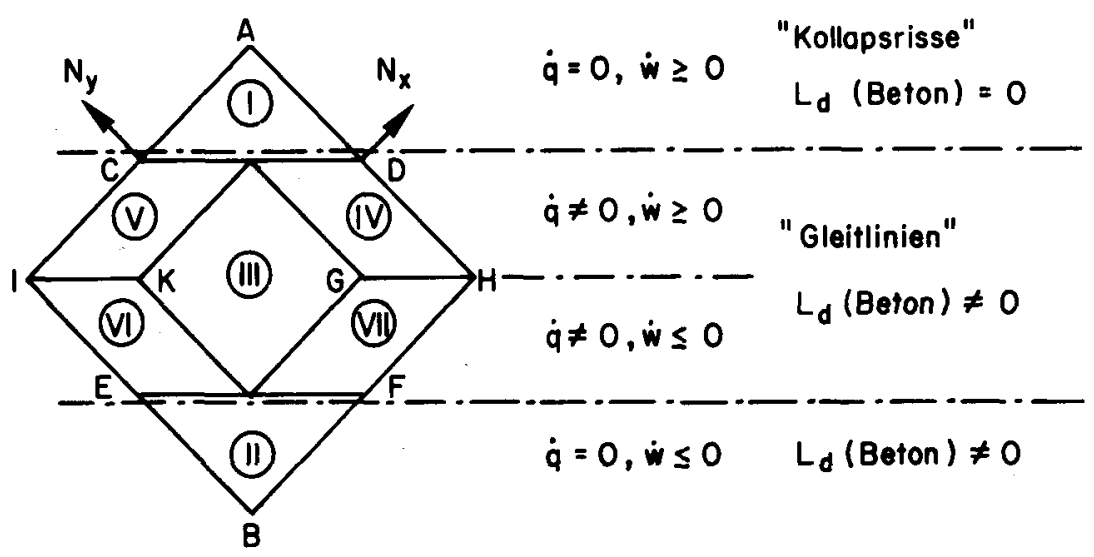

Fig. 3.8 Zuordnung von Unstetigkeiten und Fliessregimes 


\section{ERGAENZUNGEN}

\subsection{Nichtorthogonale Armierung}

Für nichtorthogonale oder sogar dreilagige Armierung und kompliziertere Betonfliessfiguren wird die mathematische Formulierung sehr schnell unübersichtlich. Dagegen erlaubt die Deutung der Scheibenfliessfigur als Umhüllende aller Betonfliessfiguren mit Ursprung innerhalb der Armierungsfliessfigur sofort eine qualitative Zeichnung der Scheibenfliessfigur. Aus dieser sind das qualitative Verhalten und die massgebenden fliessgrenzen von Eeton und Stahl für spezielle Fälle sofort ersichtlich, und die mathematische Formulierung kann auf die massgebenden Fliessbedingungen beschränkt werden.

Als Beispiel sei eine zweilagige Armierung betrachtet, deren Lagen in einem Winkel von $45^{\circ}$ zueinander verlegt sind (Fig. 4.1).

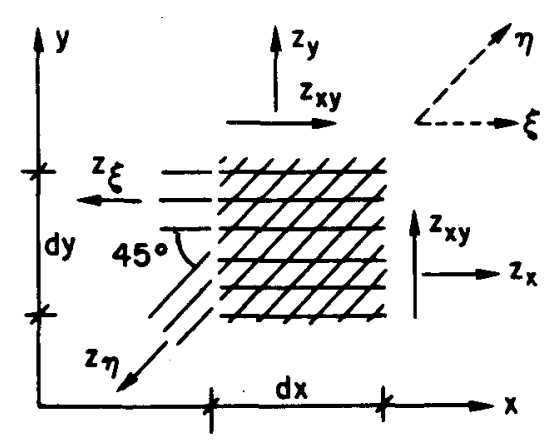

(a)

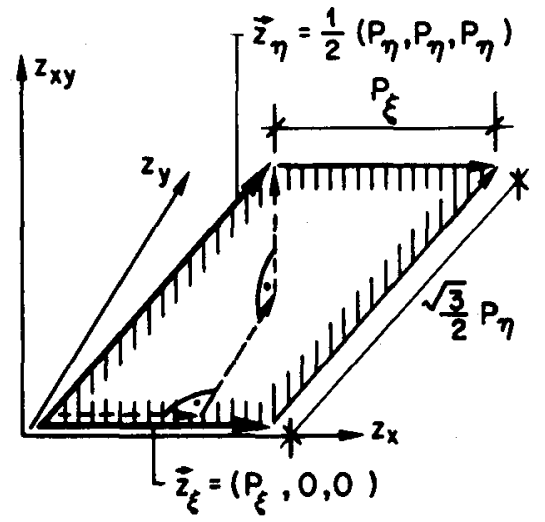

(b)

Fig. 4.1 Differentielles Armierungselement (a) und Stahlfliessfigur (b)

Wie früher werden Druckspannungen in der Armierung vernachlässigt. Die Betonfliessfigur ist weiterhin durch Fig. 2.1 gegeben. Mit $z_{\xi}, z_{n} b z w . P_{\xi}, P_{\eta}$ werden die Zug-bzw. Fliesskräfte der Armierung pro Längeneinheit senkrecht zur Armierungsrichtung bezeichnet.

Im dreidimensionalen Spannungsraum $z_{x}, z_{y}, z_{x y}$ (Fig. 4.1b) werden die Armierungsschnittkräfte $z_{\xi}, z_{\eta}$ durch die vektoren

$\vec{z}_{\xi}=k_{\xi}\left(P_{\xi}, 0,0\right), \quad \vec{z}_{n}=\frac{1}{2} k_{n}\left(P_{n}, P_{n}, P_{n}\right)$,

dargestellt, und die Fliessfiguren der Armierungslagen $\xi$ und $n$ sind durch die auf den Vektoren $\vec{z}_{\xi}, \vec{z}_{n}$ liegenden Strecken

$0 \leq{ }^{k}{ }_{\xi} \leq 1, \quad 0 \leq k_{n} \leq 1$

gegeben. Es verdient noch festgehalten $z$ u werden, dass die Vektoren $\vec{z}_{\xi}$, $\vec{z}_{\eta}$ bei allgemeiner Drientierung der Armierungslagen und des Koordinatensystems immer auf dem Kegel $z_{x y}^{2} \approx z_{x} z_{y}$ liegen und somit immer parallel zu einer Mantellinie der Betonfliesskegel sind. 
Vom Armierungsnetz können somit gesamthaft die Schnittkräfte

$\left.\begin{array}{ll}z_{x}=k_{\xi} p_{\xi}+\frac{1}{2} \kappa_{n} p_{n}, & z_{y}=z_{x y}=\frac{1}{2} \kappa_{n} p_{n}, \\ 0 \leq k_{\xi} \leq 1 & 0 \leq k_{n} \leq 1\end{array}\right\}$

aufgenommen werden, und die Fliessfigur der Armierung ist durch das in Fig. 4.1b dargestellte, von den Vektoren $\vec{z}_{\xi}, \vec{z}_{n}$ aufgespannte ebene Parallelogramm gegeben.

Einfacher geht man jedoch direkt von den durch die Strecken (4.2) dargestellten Fliessfiguren der einzelnen Armierungslagen aus und gewinnt die Scheibenfliessfigur schrittweise: zuerst wird die Betonfliessfigur mit der einen, anschliessend die neu entstandene Fliessfigur mit der Fliessfigur der zweiten Armierungslage linear kombiniert. Die Linearkombination einer konvexen Fliessfigur mit einer Strecke bzw. die Umhüllende aller dieser Fliessfiguren mit Ursprung auf der Strecke ist nämlich unmittelbar einsichtig.

Betrachtet man die Fliessfigur aus einer zur Strecke parallelen Blickrichtung, so besteht die Umhüllende aus den sichttaren bzw. unsichtbaren Teilflächen der alten Fliessfigur, wenn sich deren Ursprung im nahen bzw. fernen Endpunkt der Strecke befindet. Diese zwei Teilflächen sind durch eine Zylinderfläche verbunden, die von den den sichtbaren vom unsichtbaren Teil trennenden Konturlinien der alten fliessfigur bei der Verschiebung längs der Strecke erzeugt wird. Damit ist die Gewinnung der Linearkombination einer konvexen Fliessfigur mit einer Strecke auf das Finden der Konturlinien für eine Blickrichtung parallel zur Strecke reduziert.

Fig. 4.2 a,b zeigt die zwei mäglichen Varianten der Fliessfigur nach dem ersten Schritt. Die eingezeichneten Höhenlinien beziehen sich auf die obere Fläche. Da die Armierungsvektoren immer parallel zu einer Mantellinie der Betonfliesskegel verlaufen, sind die Kanturlinien der Betonfliessfigur immer identisch mit der Schnittkurve der zwei Kegel. Die Fliessfigur besteht somit für beliebige Armierungsrichtung aus den zwei gegeneinander verschobenen Betonfliesskegeln, die durch einen elliptischen Zylinder mit Achse parallel zum Armierungsvektor verbunden sind. In beiden Fliessfiguren sind strichpunktiert die Konturlinien eingetragen für eine Blickrichtung parallel zum noch nicht berücksichtigten Armierungsvektor. Sie trennen die sichtbaren und unsichtbaren Teilflächen für den zweiten Schritt, welcher unmittelbar die gesuchte Fliessfigur (Fig. 4.2 c) liefert. Die Fliessflächen I und IV bzw. II und VI sind identisch mit den sichtbaren bzw. unsichtbaren Teilflächen der Fliessfigur 4.2 a, wenn sich deren Ursprung in den Punkten $k_{\xi}=1\left(N_{x}=P_{\xi}, N_{y}=N_{x y}=0\right) b z w . K_{\xi}=O\left(N_{x}=N_{y}=N_{x y}=0\right)$ befindet.

Die Fliessflächen III, V, VII dagegen werden von der Konturlinie der Fliessfigur 4.2 a erzeugt, wenn deren Ursprung von $k_{\xi}=0$ nach $k_{\xi}=1$ wandert. Entsprechendes gilt, wenn von Fliessfigur 4.2 b ausgegangen wird. Genau gleich werden die in Fig. 4.2 nicht gezeichneten unteren Fliessflächen hergeleitet. Die Erweiterung der Fliessfigur in einem dritten Schritt für dreilagige Armierung bietet ebenfalls keine Probleme.

Der Vergleich der Figuren 2.5 und 4.2 c zeigt, dass sich die Fliessfiguren für orthogonale und nichtorthogonale Armierung wohl geometrisch, nicht aber in ihrem strukturellen Aufbau unterscheiden, solange mit der quadratischen Betonfliessfigur gearbeitet wird. Wie bei ortho: gonaler Armierung besteht die Fliessfigur aus den zwei gegeneinander verschobenen Betonfliesskegeln I, II, die durch vier Zylinderflächen IV - VII und zwei Ebenen III verbunden sind. Auch die Charakterisierung der einzelnen Fliessflächen im Abschnitt 2.3 gilt vollumfänglich weiter, wobei in Fig. $2.6 z_{x}, P_{x}, z_{y}, P_{y}$ durch $z_{\xi}, P_{\xi}, z_{\eta}, P_{\eta} z u$ ersetzen und die letzte Be- 
ziehung für Fliessfläche III zu streichen ist. Insbesondere befindet sich der Beton in allen Fliessregimes ausser II in einem einaxialen Druckspannungszustand. Dies erklärt sich geometrisch durch die Tatsache, dass die Armierungsvektoren immer parallel zu einer Mantellinie des Betondruckfliesskegels sind, und die Konturlinien auf dem Druckfliesskegel nie ausserhalb der Schnittkurve mit dem Zugfliesskegel verlaufen können.

Die Kinematik ist nur in den Fliessregimes III - VII verändert, wo nun die Dehnungsgeschwindigkeiten in nichtorthogonalen Richtungen gebunden sind. Dies bedeutet, dass nun in jedem der Regimes III - VII $\dot{\varepsilon}_{1}+\dot{\varepsilon}_{2}=\dot{\varepsilon}_{x}+\dot{\varepsilon}_{y}$ grösser oder kleiner null sein kann und somit sowohl Unstetigkeiten (3.16a) als auch (3.16b) vorkommen können. Diese Tatsache ist ebenfalls dafür verantwortlich, dass sich bei Verwendung einer wirklichkeitsnaheren Betonfliessfigun auch strukturelle Unterschiede zwischen den Scheibenfliessfiguren für orthogonale und nichtorthogonale Armierung ergeben.

Schliesslich ist festzuhalten, dass die für die Plattenfliessbedingung von Johansen verwendbare Methode der "äquivalenten orthogonalen Ersatzarmierung" [31] nur die Fliessflächen I und II richtig erfasst, wo beide Armierungslagen gleichzeitig an der gleichen Fliessgrenze liegen.

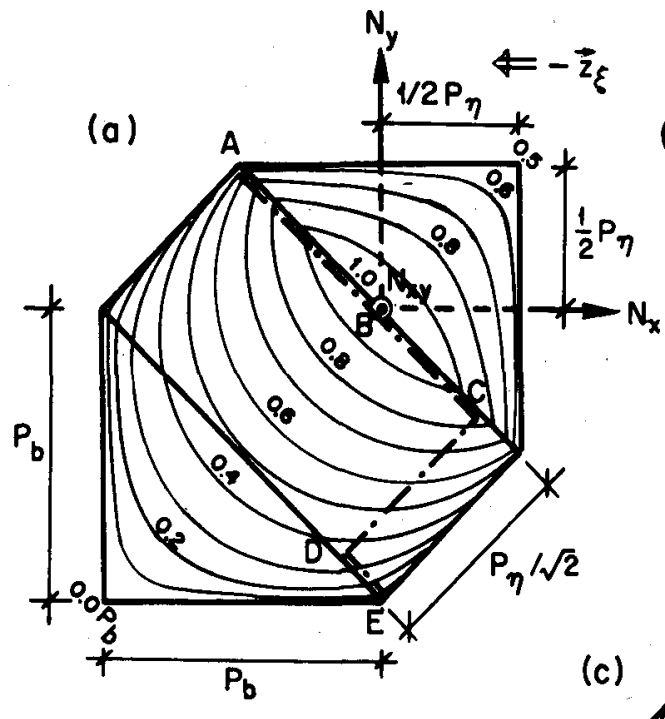

$$
\begin{aligned}
& \text { (a) : Beton + Loge } \eta \\
& \text { (b) : Beton + Loge } \xi \\
& \text { (c) : Beton + Loge } \xi+\eta \\
& P_{\xi}=0.5 P_{b}, P_{\eta}=P_{b} \\
& \text { Aequidistonz }=0.1 P_{b}
\end{aligned}
$$

- Konturlinien für Blickrichtung $\Rightarrow$ $d=\frac{1}{2} P_{b}\left(1+\frac{1}{2} \sqrt{2}\right)-\frac{1}{2} P_{\eta}$

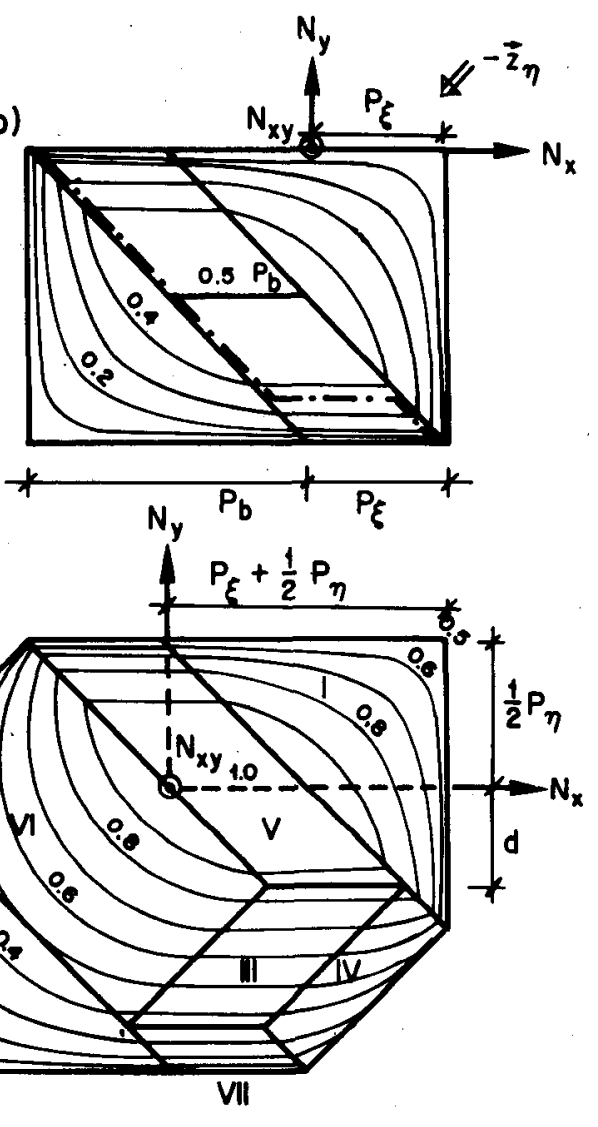

Fig. 4.2 Fliessfigur für Stahlbetonscheiben mit nichtorthogonaler Armierung 
Die im letzten Abschnitt verwendete geometrische Methode zur Gewinnung der Scheibenfliessfigur erlaubt auch sofort eine qualitative Beurteilung des Einflusses einer wirklichkeitsnaheren Erfassung der zweiaxialen Betonfestigkeit.

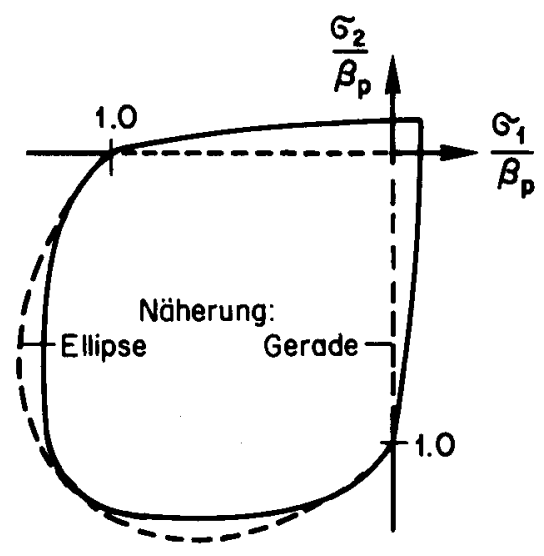

(a)

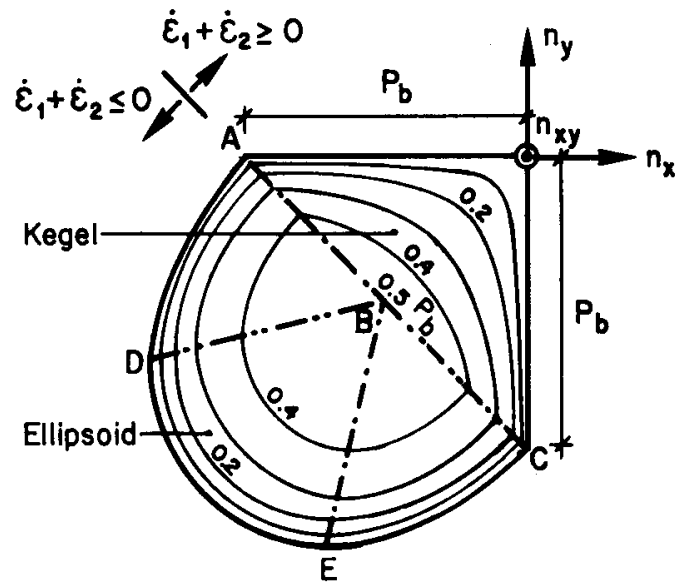

(b) $=::=$ Konturlinien

Fig. 4.3 (a) Zweiaxiale Festigkeit des Beton nach [32]

(b) Näherung im dreiaxialen Spannungsraum

Fig. 4.3 a zeigt die von Kupfer et. al. [32] gemessene zweiaxiale Betonfestigkeit. Da bei armiertem Beton der Aufbau von grösseren Stahlspannungen mit dem weitgehenden Abbau der Betonzugfestigkeit verbunden ist, ist diese weiterhin null zu setzen. Für eine qualitative Betrachtung genügt es, im Druckquadranten mit einer Ellipse zu arbeiten, deren Zentrum in guter Näherung im Punkt ( $-\frac{1}{2},-\frac{1}{2}$ ) angenommen werden darf. Die resultierende Beton"fliess"figur im dreidimensionalen Spannungsraum ist in Fig. 4.3 b in Höhenliniendarstellung gezeichnet. Eingetragen sind ebenfalls die Konturlinien für die Blickrichtungen $n_{x}$ (ABE) und $n_{y}$ (CBD). Sie bestehen je aus zwei in verschiedenen Vertikalebenen liegenden Ellipsenhälften und verlaufen nun auch in zu zweiaxialen Druckspannungszuständen gehörenden Gebieten. Die entsprechende Stahlbetonscheibenfliessfigur für orthogonale Armierung ist in Fig. 4.4 dargestellt.

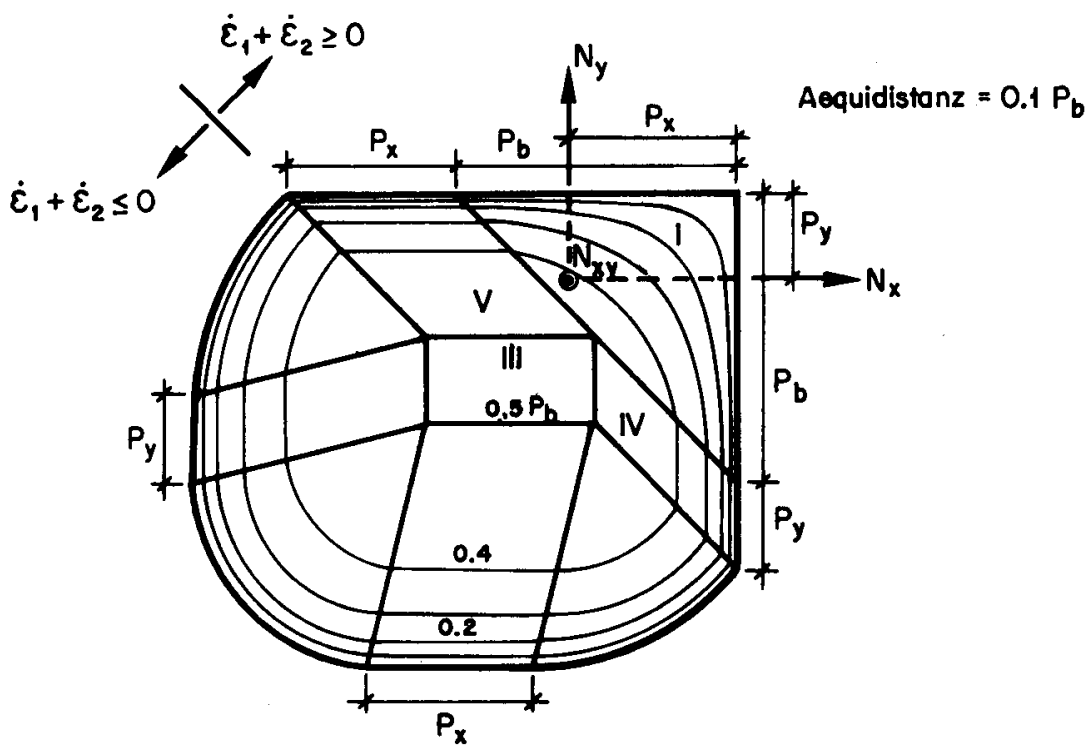

Fig. 4.4 Fliessfigur für Stahlbetonscheiben mit orthogonaler Armierung und Betonfestigkeit gemöss Fig $4.3 \mathrm{~b}$ 
Sie besteht aus den entlang den Konturlinien aufgetrennten und gegeneinander verschobenen Fliessflächen der Betonfliessfigur, die durch vier Zylinderflächen und zwei Ebenen verbunden sind. Letztere werden in den beiden Schnittpunkten der Konturlinien eingeschoben. Eine Berücksichtigung der Armierung auf Druck würde wieder nur die Länge der Zylinderflächen bzw. die entsprechende Ausdehnung der Ebenen in Richtung der negativen $N_{x}-$, $N_{y}$-Achsen verdoppeln.

Die Fliessregimes I, III, IV, V, bei welchen für keine Armierungslage die Druckfliessgrenze massgebend wird, bleiben also gegenüber der Fliessfigur 2.5 unverändert. Insbesondere befindet sich der Beton in diesen Regimes in einem einaxialen Spannungszustand. Kinematisch ist dies leicht verständlich, folgt doch für orthogonale Armierung aus $\dot{\varepsilon}_{x} \geq 0, \dot{\varepsilon}_{y} \geq 0$ sofort $\dot{\varepsilon}_{x}+\dot{\varepsilon}_{y}=\dot{\varepsilon}_{1}+\dot{\varepsilon}_{2} \geq 0$, und der Beanspruchungspunkt des Betons kann nur auf dem Zugfliesskegel der Betonfliessfigur 4.3 b liegen. In den Fliessregimes, die in Fig. 3.2 den Kanten IK, $\mathrm{GH}$ und den Fliessflächen VI, VII entsprechen, und in denen die eine Armierungslage an der Druckfliessgrenze, die andere an der Zugfliessgrenze bzw. zwischen Zug- und Druckfliessgrenze liegt, befindet sich der Beton nun dagegen neu in einem zweiaxialen Druckspannungszustand.

Bei nichtorthogonaler Armierung kann auch für $\dot{\varepsilon}_{\xi} \geq 0, \dot{\varepsilon}_{\eta} \geq 0 \dot{\varepsilon}_{1}+\dot{\varepsilon}_{2} \leq 0$ sein. Bei Verwendung der wirklichkeitsnaheren Betonfliessfigur 4.3. b gehören zu diesen Hauptdehnungsgeschwindigkeiten zweiaxiale Druckspannungszustände. Die Fliessfiguren für orthogonale und nichtorthogonale Armierung unterscheiden sich deshalb - anders als bei Verwendung der quadratischen Betonfliessfigur - auch im strukturellen Aufbau. Gegenüber der Fliessfigur 4.2 c bleiben nur diejenigen Bereiche der Regimes III, IV, V unverändert, für die $\dot{\varepsilon}_{1}+\dot{\varepsilon}_{2} \geq 0$ gilt. Insbesondere kann sich der Beton auch in einem zweiaxialen Druckspannungszustand befinden, wenn beide Armierungslagen auf Zug fliessen. Die Bereiche der Beanspruchung $N_{x}, N_{y}, N_{x y}$, für die die Fliessfigur 4.2 c nicht mehr zutrifft, sind aus der qualitativ sofort angebbaren modifizierten Fliessfigur 4.6 ersichtlich. Man gewinnt sie am einfachsten wieder geometrisch.

Fig. 4.5 zeigt qualitativ die Konturlinien der Betanfliessfigur für Blickrichtungen parallel zu den negativen Armierungsvektoren $\vec{z}_{\xi}$, $\vec{z}_{n}$ für zwei mögliche Lagen (a) und (b) des Koordinatensystems $(x, y)$. Die Armierung entspricht wieder der Fig. 4.1 a.

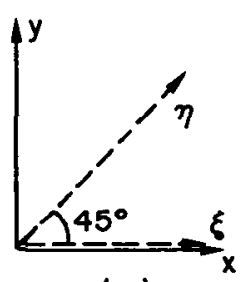

(a)

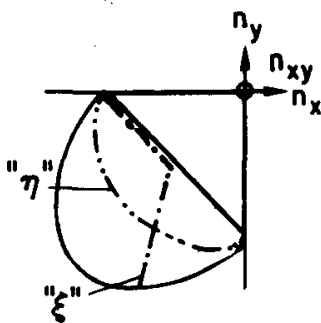

"६"

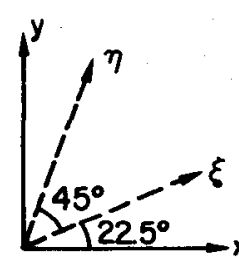

(b)

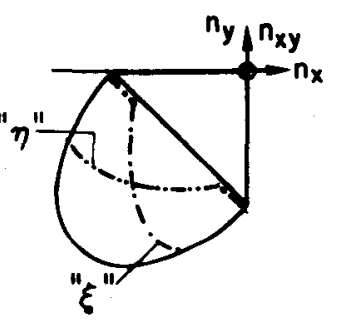

Fig. 4.5 Konturlinien der Betonfliessfigur für Blickrichtungen $-\vec{z}_{\xi},-\vec{z}_{\eta}$

Im Unterschied zu Fig. 4.3 b liegt nun die eine der zwei eine Konturlinie bildenden Halbellipsen nicht mehr in einer vertikalen, sondern in einer schiefen Ebene. Die obere Hälfte der resultierenden Scheibenfliessfigur ist rein qualitativ in Fig. 4.6 angedeutet. Die Fliessfigur gilt für das Koordinatensystem gemäss Fig. 4.5 a, um den Vergleich mit Fig. 4.2 c zu ermöglichen. Sie kann direkt auf mit schiefen Bügeln armierte Träger angewendet werden. Für ein Koordinatensystem gemäss Fig. 4.5 b wäre sie jedoch symmetrisch zur Ebene $N_{x}=N_{y}$.

Einander entsprechende Fliessregimes in den figuren 4.6 und $4.2 \mathrm{c}$ sind gleich numeriert. Insbesondere gilt die Charakterisierung der einzelnen Fliessregimes in Abschnitt 2.3 durch die Spannungszustände im Stahl sinngemäss weiter. In den mit gestrichenen Ziffern bezeichneten 
Fig. 4.6 Fliessfigur für Stahlbetonscheiben mit nichtorthogonaler Armierung und Betonfestigkeit gemöss Fig. $4.3 \mathrm{~b}$

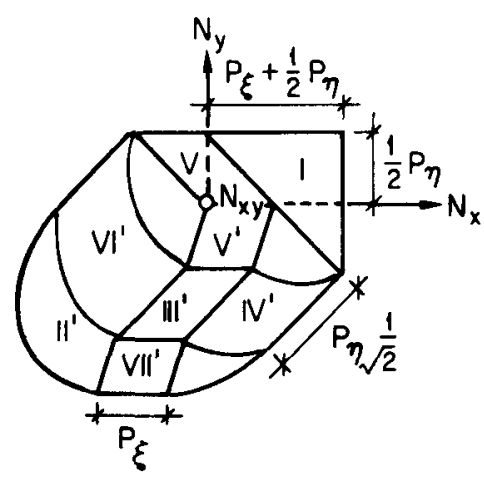

Regimes befindet sich der Beton nun jedoch neu in einem zweiaxialen Druckspannungszustand. Den unnumerierten Fliessflächen in Fig. 4.6 entsprechen in Fig. 4.2 c Kanten. In den Regimes IV', V' fliesst je eine, auf der Fliessfläche zwischen den Regimes I, IV', V' beide Armierungslagen, während sich der Beton in einem zweiaxialen Druckspannungszustand befindet.

Aus Fig. 4.6 lässt sich sofort ablesen, wann bei einem konkreten Problem mit der wirklichkeitsnaheren Betonfliessfigur gearbeitet werden sollte. 
Von Peter [33] wurden in zwei Versuchsreihen $160 \times 160 \times 20 \mathrm{~cm}$ messende Scheiben mit orthogonaler Armierung unter einachsiger Zugbeanspruchung geprüft. Diese Versuche sind sehr ausführlich und in vollständiger theoretischer Uebereinstimmung mit der vorliegenden Arbeit von Baumann [16] kommentiert worden. Es wird auf eine Wiederholung dieses ausgezeichneten Kommentars verzichtet. Im folgenden werden die wichtigsten Ergebnisse wiedergegeben und einige zusätzliche Feststellungen angefügt.

In einer ersten Versuchsreihe wurde der Einfluss der Richtungsabweichung des volumengleichen Armierungsnetzes $\left(\mu_{x}=\mu_{y}=0.6 \%\right)$ von der Hauptspannungsrichtung untersucht. Die Abweichung von Armierungs - und Hauptzugrichtung wurde in Stufen von $10^{\circ}$ von $0^{\circ}$ bis $40^{\circ}$ variiert. In zwei weiteren Versuchen (S2r20/0.2; S2r30/0.5) mit $20^{\circ}$ bzw. $30^{\circ}$ Richtungsabweichung der Hauptarmierung von der Hauptzugrichtung wurde die Querarmierung auf $1 / 5$ bzw. $1 / 2$ reduziert.

In allen Versuchsscheiben ist natürlich das Fliessregime I massgebend. Die grösste Abweichung zwischen theoretischer und experimenteller Kollapslast beträgt $7 \%$ [16]. Besonders interessant ist der Versuch 52r20/0.2. Dieser Versuch wurde von Peter besonders kommentiert [33], weil der Bruch der Scheibe nicht entsprechend seiner Theorie - und wie in der ersten Versuchsreihe beobachtet - entlang eines zur Hauptzugrichtung senkrechten Risses erfolgte, sondern der Bruchriss eine Neigung von rund $50^{\circ}$ gegenüber der Hauptzugrichtung aufwies. Diese Richtung stimmt aber ziemlich genau mit der theoretischen Drucktrajektorienrichtung im Kollapszustand, d.h. mit der Kollapsrissrichtung, überein. Nur bei volumengleicher Armierung steht die Kollapstrajektorienrichtung senkrecht auf der Hauptzugrichtung.

Unter Annahme eines zugspannungslosen Betonspannungszustandes und übereinstimmender Hauptaxen in Beton und Stahl berechnet Baumann [16] für den elastischen Bereich der Armierung die theoretische Drucktrajektorienneigung und das theoretische Spannungsverhältris zwischen den beiden Armierungsrichtungen. Die beobachtete Rissrichtung liegt immer im Bereich, der von der Anrissrichtung und den Drucktrajektorienrichtungen im elastischen Bereich der Armierung bzw. im Kollapszustand begrenzt wird. Dabei ist zu beachten, dass die "elastische" Trajektorienrichtung auch bei volumengleicher Armierung nicht mehr mit der Senkrechten zur Hauptzugrichtung zusammenfällt. Sobald sich das Rissebild ausgebildet hat, stimmen die experimentell ermittelten Spannungsverhältnisse zwischen Haupt- und Querarmierung ebenfalls sehr gut mit dem theoretischen Verlauf in Funktion der Richtungsabweichung des Armierungsnetzes überein. Diese Ergebnisse zeigen [16], dass sich der Beton schon im elastischen Bereich der Armierung durch Spannungsumlagerungen und Bildung neuer Risse der Aufnahme von Schub- bzw. Zugspannungen entzieht.

Aus der Extrapolation der gemessenen Stahldehnungen bzw. - spannungen [33] lässt sich der Schluss ziehen, dass die Querarmierung beim Kollaps die Fliessspannung nur bei den beiden volumengleich armierten. Scheiben mit Richtungsabweichung von $10^{\circ}$ bzw. $20^{\circ}$ nicht erreicht hat, bei welchen die Drucktrajektorien beim Kollaps theoretisch nur $10^{\circ} \mathrm{bzw}$. $20^{\circ}$ gegen die Querarmierung geneigt sind. Dagegen hat die Querarmierung in der erwähnten Scheibe $52 \mathrm{r} 20 / 0.2 \mathrm{mit}$ ebenfalls $20^{\circ}$ Richtungsabweichung des Armierungsnetzes die Fliessspannung eindeutig erreicht. Beim Kollaps ist hier die Drucktrajektorie theoretisch rund $60^{\circ}$ zur Querarmierung geneigt. Die Versuche von Peter [33] lassen somit auf eine gleiche Grenzneigung der Drucktrajektorien gegenüber der Armierung schliessen, wie sie von Lampert [18] angegeben worden ist $(0.5 \leq \tan \alpha \leq 2)$. Die beiden Scheiben, bei welchen die Querarmierung die Fliessgrenze nicht erreicht hat, weisen einen Winkel zwischen "elastischer" und "plastischer" Drucktrajektorienneigung von $20^{\circ}$ bzw. $16^{\circ}$ auf, während dieser Wert bei den übrigen Scheiben $10^{\circ}, 3^{\circ}, 7^{\circ}, 4^{\circ}$ 
beträgt. Daraus lässt sich der Schluss ziehen, dass vor allem die Spannungsumlagerungen nach Fliessbeginn der ersten Armierungsrichtung beschränkt sind.

In Abschnitt 8 wird gezeigt, dass die aus dem Fachwerkmodell gewonnenen Interaktionsformeln für Stahlbetonträger mit dünnwandigem, geschlossenem Querschnitt unter konstantem Torsionsund Biegemoment die theoretisch exakte Traglast aufgrund der Fliessbedingung (2.7 I) liefern. Die in [18, 34-36] nachgewiesene gute Uebereinstimmung zwischen Theorie und Experiment bestätigt deshalb ebenfalls die Fliessbedingung ( 2.7 I).

Zur experimentellen Ueberprüfung der Fliessregimes III, IV, V muss auf Schubversuche an I-Trägern zurückgegriffen werden, bei welchen der Bruch durch Versagen des Stegbetons eingeleitet wurde. Diese werden im Anschluss an die Theorie im Abschnitt 7.3 kommentiert. Da diese Versuche jedoch nicht speziell für den vorliegenden Zweck konzipiert wurden, fehlen zum Teil für die Interpretation wesentliche Materialkennwerte und Messungen. Zudem lässt sich auch der Einfluss der Druckflanschen nicht eruieren. 
Die Berechnung der Traglast aufgrund der Balkentheorie setzt die Kenntnisse von Fliessbedingungen für das differentielle Balkenelement, d.h. von Querschnittsinteraktionsbeziehungen, voraus. Zur Ableitung von Querschnittsinteraktionsbeziehungen müssen immer vereinfachende Annahmen bezüglich des Spannungs- und Verformungszustandes des Querschnittes getroffen werden.

Für unterarmierte Stahlbetonbalken unter Torsion, Biegung und Querkraft, bei welchen der Kollaps durch Fliessen der Armierung eingeleitet wird, stellt das Fachwerkmodell [18 - 23] einen solchen (statischen) Ansatz dar. Die Gültigkeit der mit dem Fachwerkmodell abgeleiteten Interaktionsbeziehungen ist durch Versuchsresultate gut belegt [34 - 36]. Das Modell bildet deshalb heute in verschiedenen Stahlbetonnormen die rationale Grundlage für die Bruchbemessung der Armierung [25, 29, 30].

Die theoretischen Grundlagen dieser Interaktionsbeziehungen sind aber noch nicht vollständig. Plastizitätstheoretisch strenge, obere Grenzwerte und mit dem getroffenen Spannungsansatz verträgliche Mechanismen sind bisher noch nicht angegeben worden. Damit ist insbesondere auch unbekannt, mit welchem Verformungsansatz der Balkentheorie der Fachwerkmodellansatz verträglich ist. Es fragt sich auch, ob die Anwendbarkeit der Plastizitätstheorie auf unterarmierte Stahlbetonbalken beschränkt ist.

Die folgenden drei Abschnitte befassen sich deshalb mit der Weiterentwicklung der theoretischen Grundlagen der plastischen Berechnung von dünnwandigen Stahlbetonbalken unter Torsion, Biegung und Querkraft. Das Schwergewicht liegt dabei einerseits auf den Kollapsmechanismen und andererseits auf dem Einbezug von Fliessregimes mit massgebender Betonfestigkeit.

\section{BALKEN IM FLIESSREGIME I: FACHWERKMODELL UND BALKENTHEORIE}

Das Fliessregime I ist für die Praxis von besonderer Bedeutung, weil bei der Bemessung der Armierung und der Abmessungen schubbeanspruchter Stege ein Versagen im Regime I angestrebt wird, d.h. ein Versagen, das durch Fliessen beider Armierungslagen ausgelöst wird. Dies garantiert einerseits eine optimale Ausnützung des Stahles und damit Wirtschaftlichkeit und andererseits einen duktilen sich durch starke Rissebildung ankündigenden Bruch. Diese "Philosophie" liegt auch dem Fachwerkmodell mit optimaler Diagonalenneigung für unterarmierte Stahlbetonbalken zugrunde. Bevor das Modell diskutiert wird, sollen jedoch die Beziehungen des Fliessregimes I noch etwas anders dargestellt werden.

\subsection{Parametrische Darstellung von Fliessfläche und Fliessgesetz im Regime I}

Gemäss Fig. 2.6 sind die Spannungszustände in Beton und Stahl im Fliessregime I charakterisiert durch

$$
\begin{array}{lll}
n_{x y}^{2}=n_{x} n_{y} ; & n_{x} \leq 0, & n_{y} \leq 0 \\
z_{x}=P_{x} ; & z_{y}=P_{y} . & \text { (6.1a) }
\end{array}
$$


Einsetzen von (6.1a) in die Beziehung für die Richtung der Betondrucktrajektorien ergibt:

$\tan 2 \alpha=\frac{-2 n \frac{x y}{n_{y}-n_{x}}}{n^{-}}=\frac{2\left(\frac{n_{x y}}{n_{y}}\right)}{\left(\frac{n_{x y}}{n_{y}}\right)^{2}-1}=\frac{2\left(\frac{n_{x y}}{n_{x}}\right)}{1-\left(\frac{n_{x y}}{n_{x}}\right)^{2}}=\frac{2 \tan \alpha}{1-\tan ^{2} \alpha}$,

$n_{x y}=n_{x} \tan \alpha ; \quad n_{x y}=n_{y} \cot \alpha$,

wobei das Vorzeichen von $\alpha$ aus Fig. 3.1 ersichtlich ist. Mit (2.1) und (6.1b) folgt sofort die parametrische Darstellung der Fliessfläche I

$$
\begin{gathered}
-N_{x y}=\left(P_{x}-N_{x}\right) \tan \alpha ; \quad-N_{x y}=\left(P_{y}-N_{y}\right) \cot \alpha, \\
\tan \alpha=\sqrt{\frac{P_{y}-N_{y}}{P_{x}-N_{x}}}, \\
P_{x}-N_{x} \geq 0 ; \quad P_{y}-N_{y} \geq 0 .
\end{gathered}
$$

Genau gleich lässt sich die aus dem Fliessgesetz (3.2) resultierende kinematische Bindung $\left(\frac{\dot{\gamma}_{x y}}{2}\right)^{2}=\dot{\varepsilon}_{x} \dot{\varepsilon}_{y} ; \quad \dot{\varepsilon}_{x} \geq 0 ; \quad \dot{\varepsilon}_{y} \geq 0$,

durch Einsetzen in die Beziehung für die Richtung der kleineren Hauptdehnungsgeschwindigkeit parametrisch darstellen:

$$
\begin{aligned}
& \tan 2 \alpha=\frac{-\dot{\gamma}_{x y}}{\dot{\varepsilon}_{y}-\dot{\varepsilon}_{x}}=\frac{2\left(-\frac{\dot{\gamma}_{x y}}{2 \dot{\varepsilon}_{x}}\right)}{\left(-\frac{\dot{\gamma}_{x y}}{2 \dot{\varepsilon}_{x}}\right)^{2}-1}=\frac{2\left(-\frac{\dot{\gamma}_{x y}}{2 \dot{\varepsilon}_{y}}\right)}{1-\left(-\frac{\dot{\gamma}_{x y}}{2 \dot{\varepsilon}_{y}}\right)^{2}}=\frac{2 \tan \alpha}{1-\tan ^{2} \alpha}, \\
& -\dot{\gamma}_{x y}=2 \dot{\varepsilon}_{y} \tan \alpha ; \quad-\dot{\gamma}_{x y}=2 \dot{\varepsilon}_{x} \cot \alpha, \\
& \tan \alpha=\sqrt{\frac{\dot{\varepsilon}_{x}}{\dot{\varepsilon}_{y}}}, \\
& \dot{\varepsilon}_{y} \geq 0 ; \quad \dot{\varepsilon}_{x} \geq 0 \text {. }
\end{aligned}
$$

Durch Addition der Gleichungen (6.5a) ergibt sich noch

$$
-\dot{\gamma}_{x y}=\dot{\varepsilon}_{y} \tan \alpha+\dot{\varepsilon}_{x} \cot \alpha
$$

Zwei Gleichungen von (6.3a,b) bzw. von (6.5a,b) oder (6.6) ersetzen also äquivalent die Gleichungen für die Fliessfläche $I$, bzw. die erste Beziehung der kinematischen Bindung (6.4). Ein Spannungszustand (6.3) und ein Bewegungszustand (6.5) sind miteinander verträglich, wenn die beiden Parameter $\alpha$ übereinstimmen.

Für über den Steg eines schubbeanspruchten Balkens konstante Spannungszustände $N_{x}$, $N_{x y}$, $N_{y}=0$ wird die parametrische Darstellung (6.3) der Fliessfläche I üblicherweise als Fachwerkmodell mit optimaler Diagonalenneigung a gedeutet. Insbesondere besagt dann (6.3b), dass 
die "Druckdiagonalen"-Richtung aus der Bedingung bestimmt wird, dass $x$ - und y-Armierung fliessen. Im nächsten Abschnitt wird jedoch gezeigt, dass das Konzept Makromodell Fachwerk wohl sehr anschaulich das Kräftespiel, nicht aber die Kinematik der plastifizierten Stahlbetonscheibe erfasst.

Die Einführung der Richtung a als Parameter in Fliessfläche und Fliessgesetz ist jedoch natürlich auf jeden Fall mathematisch sinnvall, da sie als Hauptrichtung des Betonspannungszustandes und der Verzerrungsgeschwindigkeiten charakteristische Richtung des Differentialgleichungssystems der im Regime I plastifizierten Stahlbetonscheibe ist (Abschnitt 9).

\subsection{Fachwerkmodell}

Bereits vor mehr als einem halben Jahrhundert ist von Ritter und Mörsch zur Erklärung des Tragverhaltens von gerissenen auf Schub beanspruchten Stahlbetonbalken das Fachwerkmodell eingeführt worden: Druckflansch, Längsarmierung und Bügel eines I-, T- oder a-Trägers werden als Obergurt, Untergurt und Streben eines Fachwerkes aufgefasst, während dem Beton zwischen den Rissen die Funktion der Druckdiagonalen zugewiesen wird (Fig. 6.1). Die Neigung der Druckdiagonalen bestimmt sich somit aus dem Rissebild und ist meist $z u \quad 45^{\circ}$ angenommen worden.

In der Arbeit von Lampert [18], [20] wurde erstmals versucht, zur Bestimmung des Bruchwiderstandes von. Stahlbetonbalken unter Torsion und Biegung dieses Modell mit den Methoden der Plastizitätstheorie zu kombinieren. Im Sinne der Einleitung dieses Abschnittes wurde dabei angenommen, dass der Balken unterarmiert ist, d.h., dass das Versagen durch fliessen der Armierung ausgeläst wird. Dies kann in der Praxis bei der Dimensionierung der Betonabmessungen durch die Einhaltung einer empirisch ermittelten oberen Schubspannungsgrenze erreicht werden. Wesentliche und den Erfolg des "Fachwerkmodelles mit variabler Diagonalenneigung" begründende Neuerung war die Einführung der Diagonalenneigung $\alpha$ als zusätzliche freie Variable. Damit konnten die in mehreren Versuchsserien [35], [36] beobachteten Umlagerungen zwi-

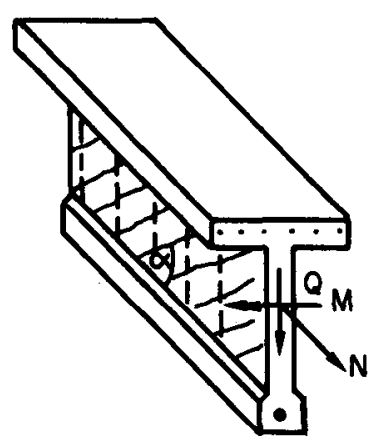

$\#$

MNQ

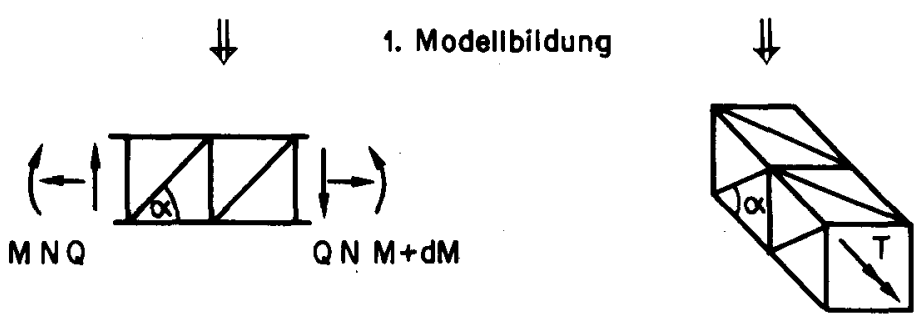

$Q N M+d M$

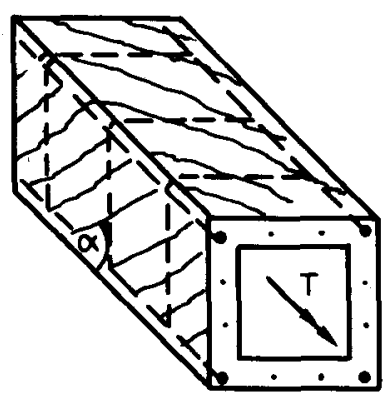

2. Formulierung von Statik und Kinematik am Modell

Fig. 6.1 Makromodell Fochwerk 
schen Bügel- und Längsarmierungskräften erfasst werden. Die grundlegenden Beziehungen dieser Arbeit sind in Fig. 6.2 sinngemäss auf das formelmässig einfachere Problem von Fig. 6.1 links übertragen. Dabei ist das Vorgehen nach dem statischen bzw. kinematischen Grenzwertsatz wie in [18] in der Tableauform einer Optimierungsaufgabe für den Traglastfaktor $\lambda$ dargestellt.

a) STATIK

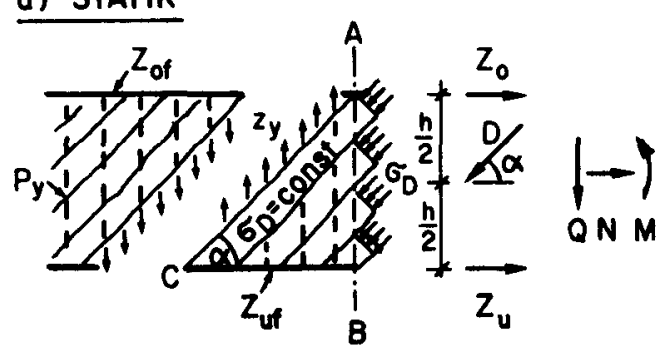

1. Schnittkröfte durch "Stabkrufte" ausdrìcken

$N=Z_{0}+Z_{u}-D \cos \alpha$

$M=\left(z_{u}-z_{0}\right) \frac{h}{2}$

$Q=D \sin \alpha$

\section{Ellmination der Betondruckkraf $f D$} als NICHTMASSGEBEND

Vertikalgleichgewicht "Free body" ABC

$$
z_{y} \cdot h \cot \alpha=D \sin \alpha
$$

$z_{y}=\frac{Q}{h} \tan \alpha \leq P_{y}$

$Z_{u}=\frac{M}{h}+\frac{N}{2}+\frac{Q}{2} \cot \alpha \leq Z_{u f}$

$Z_{0}=-\frac{M}{h}+\frac{N}{2}+\frac{Q}{2} \cot \alpha \leq Z_{\text {of }}$

\section{b) KINEMATIK}
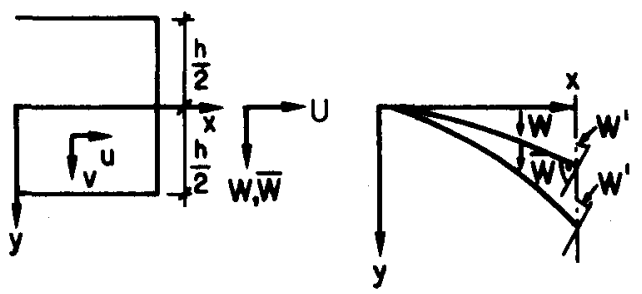

\section{Ebenbleiben der Querschnitte}

$$
\begin{array}{ll}
u=U-W^{\prime} y & v=W+\bar{W} \\
\varepsilon_{x}=U^{\prime}-W^{\prime \prime} y & =\varepsilon+x y \\
r_{x y}=\bar{W}^{\prime} & =\gamma
\end{array}
$$

\section{Kinematlk des Fochwerks mit} STARRER Druckdiogonale

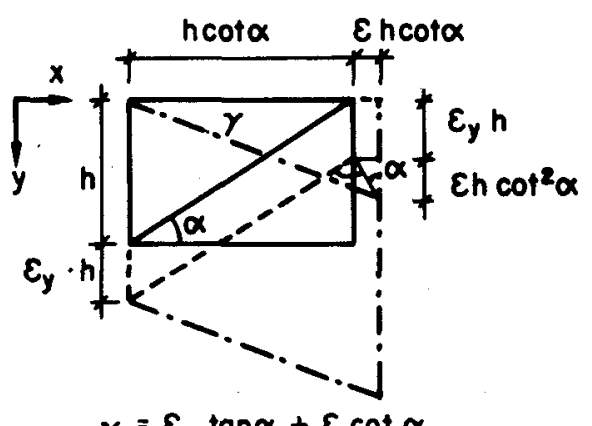

\section{TRAGLASTBERECHNUNG}

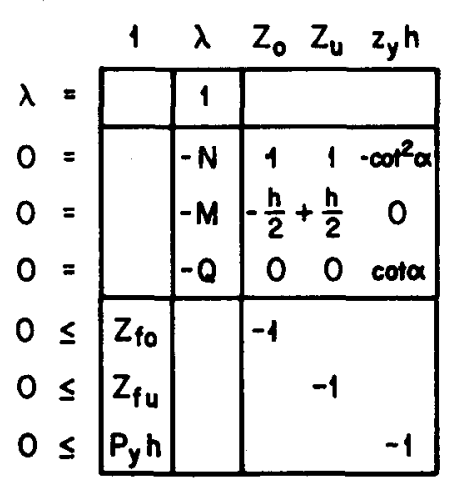

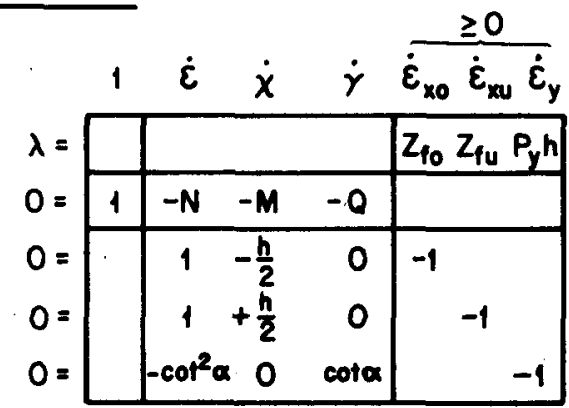

Fig. 6.2 Fochwerkmodeli sinngemäss nach [18] 
Das statische Vorgehen gemäss Fig. 6.2 a entspricht dem üblichen Vorgehen zur Ableitung einer Querschnittsinteraktionsbeziehung. Es wird das Gleichgewicht zwischen den inneren und äusseren Kräften eines Querschnittes formuliert. Dabei wird angenommen, dass der Spannungszustand im Steg konstant sei. Die Formulierung des Vertikalgleichgewichtes des Dreiecks ABC

$z_{y}=\frac{D}{h \cos \alpha} \sin ^{2} \alpha=-n_{2} \sin ^{2} \alpha=-n_{y}$

entspricht im wesentlichen der in der Balkentheorie üblichen Elimination der Normalspannung quer zur Balkenaxe, $N_{y}=z_{y}+n_{y}=0$ (siehe Abschnitt 6.3). Die Ungleichungen schliesslich fussen auf der parametrischen Darstellung (6.3) der Fliessbedingung (2.7 I).

Beim kinematischen Vorgehen gemäss Fig. 6.2 b wird die übliche Balkenannahme: "Querschnitte senkrecht zur Balkenachse bleiben eben" mit der Kinematik eines diskreten Fachwerks mit starrer Druckdiagonale (Williot'scher Verschiebungsplan) kombiniert. Hier wird deutlich, dass dem Vorgehen das Konzept Makromodell "Diskretes Fachwerk" im Sinne von Fig. 6.1 zugrunde liegt: in einem ersten Schritt wird das Tragwerk als Fachwerk idealisiert, wobei der Beton nur noch dadurch erfasst wird, dass die Fachwerkstäbe beliebig Druck aufnehmen können; in einem zweiten Schritt werden dann die Methoden der Plastizitätstheorie auf das Fachwerk angewandt.

Bei einem Fachwerk mit fester, a priori aus dem Rissebild festgelegter Geometrie, ist das Vorgehen theoretisch einwandfrei. Für festes a ergeben sich zwei lineare Programme, die tatsächlich dual sind und somit den gleichen Traglastfaktor liefern. Insbesondere entspricht jeder freien Variable im primalen statischen linearen Programm eine Restriktionsgleichung im dualen kinematischen linearen Programm. Wird nun jedoch beim statischen Vorgehen die nichtlineare Optimierungsaufgabe mit der zusätzlichen freien Variable a gelöst, so muss das zugehörige duale Problem auch eine zusätzliche dieser Variablen entsprechende Restriktion enthalten, die offensichtlich im Tableau der Fig. 6.2 b fehlt.

In der Tat: der statischen Aussage, dass in beliebiger Richtung $\alpha$ beliebiger Druck aufgenommen werden kann, entspricht die kinematische Restriktion (6.4), die in beliebiger Richtung a Stauchungen verhindert. Die Kinematik des Fachwerkes mit starrer Druckdiagonale jedoch liefert nur die Hälfte der parametrischen Darstellung dieser Restriktion, nämlich (6.6), weil nur Stauchungen in der einzigen Richtung a verhindert werden. Der Widerspruch zwischen dem Vorgehen nach Fig. 6.2 a und 6.2 b besteht also darin, dass bei der Formulierung der Kinematik am Fachwerk der Kontinuumscharakter des Betons explizit vernachlässigt wird, während dieser beim statischen Vorgehen über den als Variable behandelten Winkel a implizit eingeführt wird.

Führt man jedoch lediglich die fehlende Restriktion, z.B. in der Form (6.5b), zusätzlich ein [22], so handelt es sich theoretisch nicht mehr um ein Makromodell, weil einerseits (6.5b) am Fachwerk nicht herleitbar ist und andererseits (6.4) für das Fachwerk keine physikalische Bedeutung hat. Für einen als Scheibe aufgefassten Steg dagegen sind die Annahmen eines konstanten Stegspannungszustandes im Regime I und ebenbleibender Querschnitte nicht verträglich (siehe Abschnitt 6.3) und können nicht zur gleichen Traglast führen.

Die vorstehenden Ueberlegungen zeigen, dass mit der Einführung einer "variablen Diagonalenneigung $\alpha^{\prime \prime}$ das Konzept eines Makromodelles im Sinne von Fig. 6.1, das sowohl Statik wie Kinematik beschreibt, verlassen werden muss. Das Fachwerk erfasst wohl zutreffend und sehr anschaulich das Kräftespiel, nicht aber die Kinematik der plastifizierten Stahlbetonscheibe. Dagegen stellt das Fachwerk mit festen $45^{\circ}$-Diagonalen und damit fester Geometrie ein echtes Makromodell dar und liefert die einfachste Linearisierung der nichtlinearen Interaktionsbe- 
ziehungen.

Im folgenden Abschnitt wird gezeigt, dass sich die nichtlinearen Interaktionsbeziehungen mittels der plastischen Balkentheorie widerspruchsfrei sowohl statisch als auch über die Dissipationsleistung des Querschnittes ableiten und bestätigen lassen.

\subsection{Balkentheorie}

Wie Drucker [11] hervorgehoben hat, gibt es keine echte Interaktionsbeziehung zwischen Biegemoment und Querkraft. Es soll deshalb zuerst dargelegt werden, was im folgenden unter Balkentheorie verstanden wird und wie sie gegenüber der Scheibentheorie abgegrenzt ist.

\subsubsection{Allgemeines}

Der Uebergang von der eine zweidimensionale Tragstruktur beschreibenden Theorie des ebenen Spannungszustandes zur Balkentheorie der eindimensionalen Tragstrukturen kann durch vier Schritte charakterisiert werden, die in Fig. 6.3 zusammengefasst sind.

Auch in der plastischen Balkentheorie wird normalerweise von einer in Uebereinstimmung mit der Annahme vom Ebenbleiben der Querschnitte stehenden Definition der verallgemeinerten Verformungen ausgegangen (siehe Fig. 6.2 b)

$\varepsilon_{x}=\varepsilon+x y, \quad \gamma_{x y}=\gamma$,

wabei die verallgemeinerten Verformungen $\varepsilon, x, \gamma$ Verlängerung, Krümmung und Schiebung der Balkenaxe bezeichnen. Die Längsdehnungen weisen somit nach (6.7) einen linearen, die Schie-

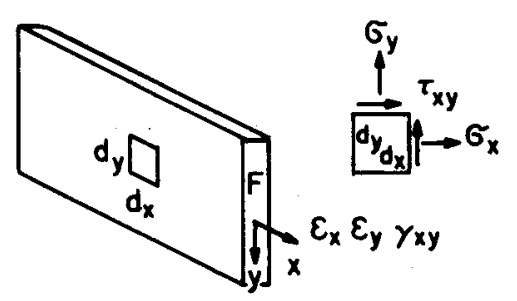

"Scheibe"

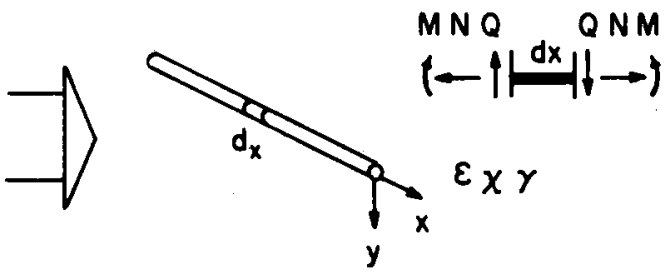

"Balken"

1. Definition verallgemeinerter Spannungen und Verformungen

$$
\begin{array}{ll} 
& N=\int \sigma_{x} d F \quad M=\int \sigma_{x} y d F \quad Q=\int \tau_{x y} d F \\
\text { 2.B. } \quad & \varepsilon_{x}=\varepsilon+x y \quad \gamma_{x y}=\gamma \\
L_{d} & =\int\left(\sigma_{x} \dot{\varepsilon}_{x}+\tau_{x y} \dot{\gamma}_{x y}\right) d F=N \dot{\varepsilon}+M \dot{x}+Q \dot{\gamma}
\end{array}
$$

\section{Elimination nichtrelevanter Spannungen}

$$
\sigma_{y}=0
$$

3. Übergang von der Fliessbedingung des differentiellen Scheibenelementes zur Fliessbedingung om differentiellen Balkenelement

4. Formulierung von Rand -, Gleichgewichts - und Kompatibilitätsbedingungen ausschliesslich in den verallgemeinerten Grossen

Fig. 6.3 Übergong "Scheibe" - "Balken". 
bungen einen konstanten Verlauf über die Balkenhöhe auf. Der Satz der verallgemeinerten Spannungen ist nun nach Prager [2] so zu wählen, dass die Dissipationsleistung des differentiellen Balkenelementes, d.h. des plastifizierten Querschnittes, als Skalarprodukt der verallgemeinerten Grössen geschrieben werden kann.

$L_{d}=\int_{F}\left(\sigma_{x} \dot{\varepsilon}_{x}+\tau_{x y} \dot{\gamma}_{x y}\right) d F=\dot{\varepsilon} \int_{F} \sigma_{x} d F+\dot{X} \int_{F} \sigma_{x} y d F+\dot{\gamma} \int_{F} \tau_{x y} d F=\dot{\varepsilon} N+\dot{X} M+\dot{\gamma} Q$

Die Beziehungen, die die verallgemeinerten Spannungen N, M, Q mit den normalen Spannungen verknüpfen, entsprechen somit der üblichen Schnittkraftdefinition

$N=\int_{F} \sigma_{x} d F, \quad M=\int_{F} \sigma_{x} y d F, \quad Q=\int_{F} \tau x y d F$.

Geht man umgekehrt von (6.9) aus, folgt die Definition der verallgemeinerten Verformungen (6.7) sofort aus der Bedingung, dass (6.8) für einen beliebigen Spannungsverlauf über $y$, instesondere auch für beliebige Variationen $\delta \sigma_{x}, \delta \tau_{x y}$ gelten muss. Charakteristisch für die übliche Definition der verallgemeinerten Grössen ist also, dass der Verlauf der Dehnungen, nicht aber derjenige der Spannungen von vornherein fixiert ist.

Sind die verallgemeinerten Grössen definiert und die nichtrelevanten Spannungen aus der Scheibenfliessbedingung eliminiert, so ist der Uebergang von der Scheibenfliessbedingung zu der Fliessbedingung am differentiellen Balkenelement - d.h. zu der M-N-Q-Interaktionsbeziehung des Querschnittes - grundsätzlich nur noch ein Problem der Theorie der kanvexen Körper. Daraus geht hervor, dass die Interaktionsbeziehung erhalten wird, ohne dass je die Rand-, Gleichgewichts- oder Integrabilitätsbedingungen des ebenen Spannungszustandes berücksichtigt worden sind. Diese kommen auch beim weiteren Vorgehen nicht mehr ins Spiel, da nach Punkt 4 Fig. 6.3 die Traglastberechnung ausschliesslich in den verallgemeinerten Grössen formuliert und durchgeführt wird.

Daraus folgt, dass die physikalische Bedeutung dieses Vorgehens im Lichte der Theorie des ebenen Spannungszustandes unklar sein kann, und dass unter Umständen mit den verallgemeinerten Spannungen und Verformungen keine Spannungs- und Dehnungsfelder assoziiert werden kännen, die die Gleichgewichts- und Integrabilitätsbedingungen des ebenen Spannungszustandes erfüllen.

Solche Interpretationsschwierigkeiten ergeben sich regelmässig, sobald die Querkraft als verallgemeinerte Spannung eingeführt wird. Sie sind Ausdruck der erstmals von Drucker hervorgehobenen Tatsache, dass es aus der Sicht des ebenen Spannungszustandes keine echte M-QInteraktionskurve gibt, weil das Problem nicht auf die Betrachtung eines isolierten, vollplastifizierten, senkrecht zur Balkenaxe stehenden Querschnittes reduziert werden kann, und die Interaktionsbeziehung folglich immer von den Bedingungon in benachbarten Querschnitten abhängt $[11,12,13]$.

\subsubsection{M-N-Q-Interaktion am I-Profil im Regime I}

Es soll nun innerhalb der im vorangehenden Abschnitt abgegrenzten Balkentheorie die M-N-QInteraktionsbeziehung hergeleitet werden (Fig. 6.4 a), und zwar unter den gleichen Voraussetzungen, die normalerweise den Ausgangspunkt für die Anwendung des Fachwerkmodelles nach Fig. 6.2 a bilden. Es wird also insbesondere angenommen, dass die Steg- und Flanschscheiben des idealisierten Querschnittes nach Fig. 6.4 b nur im Regime I plastifiziert werden. Die. Fliesskraft der Bügel pro Längeneinheit sei $P_{y}$, diejenige der gesamten Flanschlängsarmierung $z_{\text {fo }}$ bzw. $Z_{f u}$. 


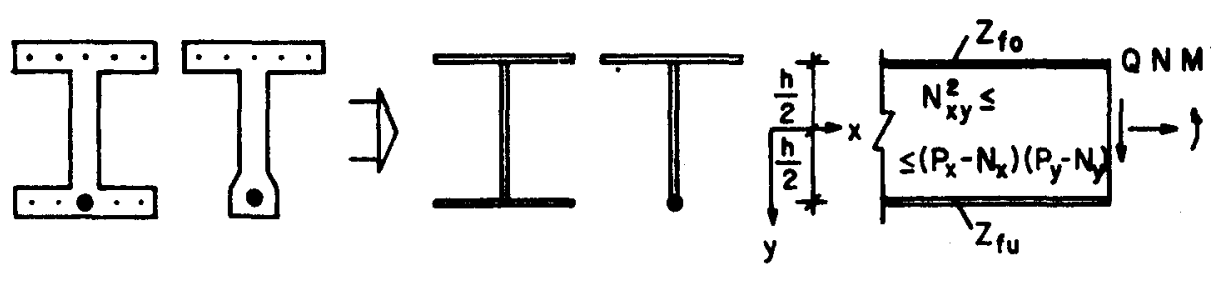

a) "Wirklichkeit"

b) Idealisierung

Fig. 6.4 Idealisierung des Querschnittes

Wesentlich für die Definition geeigneter verallgemeinerter Spannungen und Verformungen ist nun vorerst die Feststellung, dass die statischen und kinematischen Annahmen nach Fig. 6.2 aus der Sicht der Scheibentheorie widersprüchlich sind. Während beim Fachwerkmodell nach Fig. 6.2 a angenommen wird, dass im plastifizierten Querschnitt der Betonspannungszustand und damit tana konstant über die Steghöhe sei, folgt aus der Annahme ebenbleibender Querschnitte nach Fig. 6.2 b mit (6.7) und (6.5a) eine lineare Variation von tana. Spannungszustand und plastische Dehnungsgeschwindigkeiten sind also nicht verträglich. Würde andererseits bei der rein statischen Herleitung der Interaktionsbeziehung nach Fig. 6.2 a tatsächlich von verallgemeinerten Spannungen und Verformungen (6.9) und (6.7) ausgegangen, so müsste $M$ bei festgehaltenem $Q$ und $N$ für alle möglichen Spannungsverläufe maximiert werden, was zu einem Variationsproblem für den unbekannten Spannungsverlauf im Steg führt.

Nun ist jedoch bei der Definition der verallgemeinerten Verformungen zu beachten, dass die Dehnungsgeschwindigkeiten im Regime I der kinematischen Bindung (6.4) unterworfen sind: Schiebungen können nur kombiniert mit Längsdehnungen auftreten. Für nicht verschwindende und gemäss (6.7) über den Querschnitt konstante Schiebungen müssten also beide Flanschen auf Zug beansprucht sein. Da damit die Druckresultierende infolge eines Biegemomentes vom Steg allein aufzunehmen wäre, wird im allgemeinen die Annahme unhaltbar, dass der Steg sich im Fliessregime I befinde. Offenbar darf durch die dritte verallgemeinerte Verformung nur die Intensität nicht aber auch der Verlauf der Schiebungen über den Steg vorgeschrieben werden. Als dritte verallgemeinerte Verformung wird folglich die mittlere Schiebung des Querschnittes eingeführt.

$\varepsilon_{x}=\varepsilon+X y$,
$\bar{\gamma}=\frac{1}{h} \int_{h} \gamma_{x y} d y$.

Eine strenge Begründung der getroffenen Wahl (6.10b) für die veraligemeinerte Schiebung wird in Abschnitt 6.4 gegeben.

Die Definition der $\bar{\gamma}$ zugeordneten verallgemeinerten Spannung $Q$ ist wieder aus dem Ausdruck für die Dissipationsleistung abzuleiten

$$
\begin{aligned}
L_{d} & =\ldots+\int_{F} \tau_{x y} \dot{\gamma}_{x y} d F=\ldots+\int_{h} N_{x y} \dot{\gamma}_{x y} d y . \\
& =\ldots+Q \frac{\dot{q}}{\gamma} \quad=\ldots+Q \frac{1}{h} \int_{h} \dot{\gamma}_{x y} d y .
\end{aligned}
$$

(6.11) ist nur dann für einen beliebigen Verlauf von $\dot{\gamma}_{x y}$ über den Steg als Identität erfüllt, wenn $N_{x y} h=Q$ gilt, und die Beziehungen, die die verallgemeinerten Spannungen $N, M, Q$ mit den normalen Spannungen verknüpfen, lauten nun 
$N=\int_{F} \sigma_{x} d F ; \quad M=\int_{F} \sigma_{x} y d F$,
$Q=\tau_{x y} F_{\text {steg }}=N_{x y} h$.

Charakteristisch für die definierten verallgemeinerten Grössen $\bar{\gamma}$, $Q$ ist also, dass nun nicht mehr der Verlauf von $\dot{\gamma}_{x y}$, sondern derjenige der Schubspannungen $\tau_{x y}$ bzw. $N_{x y}$ von vornherein als konstant fixiert ist. Nach der Elimination der nichtrelevanten Normalspannung quer zur Balkenaxe

$N_{y}=0$

lautet die Fliessbedingung für die plastifizierte Stegscheibe

$N_{x y}^{2}=\left(P_{x}-N_{x}\right) P_{y}=-n_{x} P_{y}=n_{x} n_{y}=n_{x y}^{2}$.

Aus (6.12b) und (6.14) folgt aber wieder die Fachwerkmodellannahme eines über die Steghöhe konstanten Betonspannungszustandes. Sie steht - wie in Abschnitt 6.4 und 10.1 gezeigt wird für die Voraussetzung, dass die Querkraft und die Bügelfliesskraft in Balkenlängsrichtung nur schwach variieren und in der Querschnittsumgebung näherungsweise als konstant angenommen werden dürfen.

Die Maximierung von M bei festgehaltenem $N$, Q ergibt unter Berücksichtigung der Plastizitätsbedingungen $Z_{u} \leq Z_{u f}, Z_{0} \leq Z_{\text {of }}$ in den Flanschscheiben und von (6.14) mit $P_{x}=0$ in der Stegscheibe die bekannten zwei Interaktionsflächen des Fachwerkmodelles.

$$
\left.\begin{array}{r}
\frac{M}{h}+\frac{N}{2}+\frac{Q^{2}}{2 P_{y} h}=z_{u f}, \\
-\frac{M}{h}+\frac{N}{2}+\frac{Q^{2}}{2 P_{y} h}=z_{o f} .
\end{array}\right\}
$$

Werden miteinander verträgliche, verallgemeinerte Spannungen und Verformungen eingeführt, so müssen statische und kinematische Methode auf die gleichen Interaktionsbeziehungen führen. Dies sei im folgenden verifiziert.

Die zur konvexen Fliessfigur in der $N_{x}-N_{x y}-$ Ebene

$N_{x y}^{2} \leq\left(P_{x}-N_{x}\right) P_{y}$

gehörende Stützfunktion, die definitionsgemäss mit der Dissipationsleistung des differentiellen Elementes identisch ist, lautet

$H\left(\dot{\varepsilon}_{x}, \dot{\gamma}_{x y}\right)=P_{x} \dot{\varepsilon}_{x}+P_{y} \frac{\dot{\gamma}_{x y}^{2}}{4 \dot{\varepsilon}_{x}}=L_{d}, \quad \dot{\varepsilon}_{x} \geq 0$.

Die Dissipationsleistung des plastifizierten Querschnittes

$L_{d}^{\text {Querschnitt }}=L_{d}^{\text {Flansch }}+L_{d}^{\text {Steg }}$

ergibt sich unter Benutzung von (6.10) zu

$L_{d}^{\text {Flansch }}=\dot{\varepsilon}\left(Z_{f o}+Z_{f u}\right)+\dot{x} \frac{h}{2}\left(z_{f u}-z_{f o}\right)$, 


$$
\begin{gathered}
L_{d}^{\text {Steg }}=P_{y} \int_{-h / 2}^{+h / 2} \frac{\dot{\gamma}_{x y}^{2}}{4(\dot{\varepsilon}+\dot{x} y)} d y, \\
\dot{\varepsilon} \geq|\dot{x}| \frac{h}{2},
\end{gathered}
$$

wobei im Steg wie früher einfachheitshalber $P_{x}=0$ gesetzt wurde, (6.21) aus $\dot{\varepsilon}_{x} \geq 0$ folgt, und die Stegdissipationsleistung Funktional der noch unbekannten Funktion $\dot{\gamma}_{x y}(y)$ ist.

Gesucht ist also noch diejenige Funktion $\dot{\gamma}_{x y}(y)$, die die Dissipationsleistung unter der Nebenbedingung ( $6.10 \mathrm{~b}$ ) minimiert. Dies ist ein besonders einfaches Problem der Variations rechnung, da weder (6.20) noch (6.10b) Ableitungen der gesuchten Funktion nach y enthalten. Die Euler'sche Gleichung ist deshalb finit und nicht differentiell. Sie lautet [8], wenn $\mu$ den Lagrange Multiplikator der Nebenbedingung bezeichnet:

$$
\begin{gathered}
\frac{\partial}{\partial \dot{\gamma}_{x y}}\left(P_{y} \frac{\dot{\gamma}_{x y}^{2}}{4(\dot{\varepsilon}+\dot{x} y)}-\mu \dot{\gamma}_{x y}\right)=0, \\
\dot{\gamma}_{x y}=2 \mu \frac{\dot{\varepsilon}+\dot{x}_{y}}{P_{y}} .
\end{gathered}
$$

Durch Einsetzen von (6.22) in die Nebenbedingung (6.10b) folgt für den Multiplikator $\mu$ die Beziehung

$h \bar{\gamma}=\frac{2 \mu}{P_{y}} \int_{-h / 2}^{+h / 2}(\dot{\varepsilon}+\dot{x} y) d y=\frac{2 \mu}{P_{y}} h \dot{\varepsilon} \quad$.

$\dot{\gamma}_{x y}$ und die Dissipationsleistung ergeben sich somit zu

$\dot{\gamma}_{x y}=\frac{\dot{\bar{\gamma}}}{\dot{\varepsilon}}(\dot{\varepsilon}+\dot{x} y)$,

$L_{d}^{\text {Steg }}=P_{y} h \frac{\dot{\bar{\gamma}}^{2}}{4 \dot{\varepsilon}}$.

Aus (6.5) folgt, dass tan $\alpha$ konstant über $y$ ist

$-\tan \alpha=\frac{2 \dot{\varepsilon}_{x}}{\dot{\gamma}_{x y}}=2 \frac{\dot{\varepsilon}}{\frac{\dot{r}}{\gamma}}$,

und alle Verzerrungsgeschwindigkeiten zueinander proportional und linear verlaufen. Die Bedeutung dieser Verteilung der Verzerrungsgeschwindigkeiten wird in den Abschnitten 6.3.3 und 6.4 näher diskutiert.

Die Verifikation der Interaktionsflächen (6.15) ist nun nur noch ein Problem der Theorie der konvexen Körper, da mit der Dissipationsleistung des plastifizierten Querschnittes (6.1B), $(6.19),(6.21),(6.24)$ die Stützfunktion $H(\dot{\varepsilon}, \dot{\chi}, \dot{\bar{\gamma}})$ des gesuchten konvexen Fliesskörpers im $N-M-Q-$ bzw. $\dot{\varepsilon} \dot{\chi} \dot{\bar{\gamma}}$-Raum gefunden ist.

$$
\begin{aligned}
& H(\dot{\varepsilon}, \dot{x}, \dot{\bar{\gamma}})=H_{1}(\dot{\varepsilon}, \dot{\chi})+H_{2}(\dot{\varepsilon}, \dot{\bar{\gamma}}) \\
& H_{1}=\dot{\varepsilon}\left(Z_{f o}+Z_{f u}\right)+\dot{x} \frac{h}{2}\left(z_{f u}-Z_{f o}\right), \quad \dot{\varepsilon} \geq \frac{h}{2}|\dot{x}| \\
& H_{2}=P_{y} h \frac{\dot{\gamma}^{2}}{4 \dot{\varepsilon}}
\end{aligned}
$$


Der durch $H$ definierte konvexe Körper kann gemäss (6.26) als Linearkombination der zwei durch $H_{1}$ und $H_{2}$ definierten konvexen Mengen aufgefasst werden [2, 7]. Die im Bereich (6.21) definierte Funktion $H_{1}$ ist die Stützfunktion eines Kegels in der $M-N-E b e n e$, dessen Spitze im Punkt $N=Z_{f o}+Z_{f u}, M=\frac{h}{2}\left(Z_{f u}-Z_{f o}\right)$ liegt, und der die $M$-Achse in den Punkten $M=-h Z_{f o}$, $M=+h Z_{f u}$ schneidet (Fig. 6.5a). H 2 dagegen ist die Stützfunktion der Menge aller Punkte innerhalb einer Parabel in der N-Q-Ebene (Fig. 6.5b), denn für die Randpunkte der Menge gilt $\left.\begin{array}{l}Q=\frac{\partial H_{2}}{\partial \dot{\bar{\gamma}}}=P_{y} h \frac{\dot{\bar{\gamma}}}{2 \dot{\varepsilon}}, \\ N=\frac{\partial H_{2}}{\partial \dot{\varepsilon}}=-P_{y} h\left(\frac{\dot{\bar{\gamma}}}{2 \dot{\varepsilon}}\right)^{2}, \\ N=-\frac{Q^{2}}{P_{y} h} \quad .\end{array}\right\}$

Die Linearkombination gewinnt man nun sofort geometrisch anschaulich als Umhüllende aller in Ebenen $M=$ const liegenden Parabeln (6.27) mit Scheitelpunkt innerhalb des schraffierten Kegels (Fig. 6.5a) in der M-N-Ebene. Sie wird offensichtlich durch Teile der zwei parabolischen Zylinder (6.15) dargestellt (Fig. 6.5c).

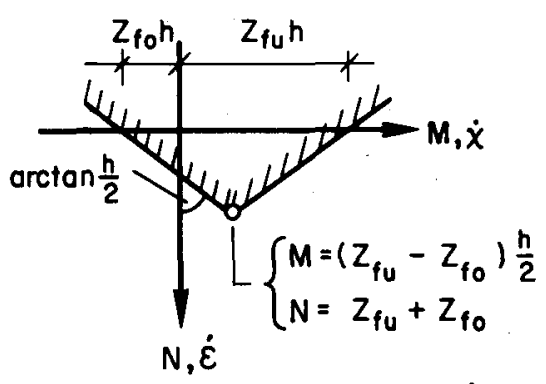

(a)

(c)

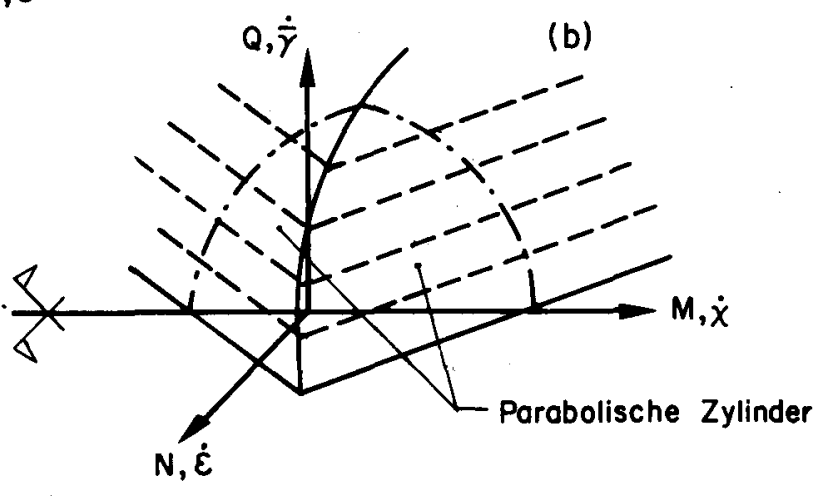

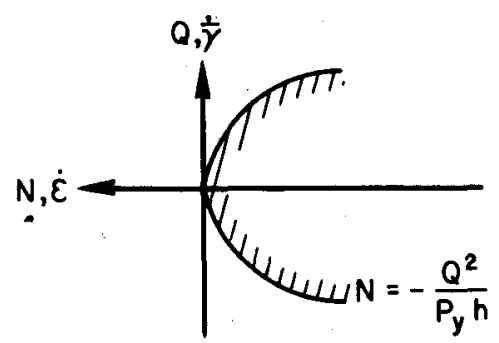

(b)

Fig. 6.5 Fliessfiguren zu den Stützfunktionen $H_{1}(a), H_{2}(b)$ und $H(c)$.

\subsubsection{Diskussion der Balkentheorie}

Abschnitt 6.3.2 zeigt, dass die "Fachwerkmodell"-Statik nach Fig. 6.2a als strenge Anwendung der plastischen Balkentheorie auf Balken mit dünnen, der Fliessbedingung (2.7 I) genügenden Stegen betrachtet werden muss. Die neue Herleitung der Interaktionsbeziehungen (6.15) über die Dissipationsleistung weist nach, dass diese innerhalb der durch die verallgemeinerten Spannungen und Verformungen (6.12), (6.10) charakterisierten Balkentheorie die exakte Fliessbedingung darstellen. Die Bedeutung dieser Balkenfliessbedingung innerhalb der Scheibentheorie jedoch ist noch unklar, da aus dieser Sicht im allgemeinen die Voraussetzungen sowohl des statischen als auch des kinematischen Grenzwertsatzes verletzt werden. 
Es ist sofort ersichtich, dass der über den Steg des plastifizierten Querschnittes konstante Spannungszustand statische Randbedindungen verletzt, sobald verteilte Belastungen auftreten, oder der Balken Vouten aufweist. Er ist ebenfalls unzulässig, wenn die Bügelarmierung im betrachteten Querschnitt in Längsrichtung ändert. Diese Verletzung von statischen Randbedingungen ist solange von untergeordneter Bedeutung, als die Grundvoraussetzungen für die Anwendbarkeit der Balkentheorie erfüllt sind: nämlich eine vernachlässigbare Variation der Querkraft, der Balkenhöhe und des Bügel- und Längsarmierungsgehaltes über Strecken in der Grössenordnung der Querschnittsabmessungen.

Andererseits weisen gemäss (6.10) die Längsdehnungsgeschwindigkeiten wohl einen linearen Verlauf auf. Der ebenfalls gemäss (6.13) lineare Verlauf der Schiebungsgeschwindigkeiten bedeutet aber, dass die Verschiebungsgeschwindigkeiten $\dot{u}$ in Richtung der Balkenaxe nicht auf einer Ebene liegen können, und der Querschnitt folglich wölbt. Zudem weist der plastifizierte Querschnitt gemäss (6.5) für tan $\neq 0$ nicht verschwindende Querdehnungsgeschwindigkeiten $\dot{\varepsilon}_{y}>0$ auf. In einem gleichmässig armierten, am freien Ende belasteten Kragträger wird nur der Einspannquerschnitt plastifiziert. Plastische Querdehnungen und Verwölbungen in einem einzigen isolierten Querschnitt bedeuten aber Verletzung von Kompatibilitätsbedingungen zwischen dem plastifizierten Querschnitt und dem starren Balkenteil bzw. der Einspannung.

Diese Verletzung von kinematischen Kompatibilitätsbedingungen ist aber nicht gleichzusetzen mit der Unverträglichkeit der Spannungs- und Dehnungsansätze in Fig. 6.2. Während die Kambination der Dehnungs- und Spannungsansätze in Fig. 6.2 auch innerhalb der Balkentheorie falsch ist, bzw. lediglich zu unteren und oberen Grenzwerten für die Balkenfliessbedingungen führen kann, sind Inkompatibilitäten wie die oben erwähnten jeglicher Balkentheorie inhärent, die über die Definition verallgemeinerter Verformungen und Spannungen als differentielles Element den Querschnitt einführt. Sie sind im vorliegenden Fall Ausdruck des Sachverhaltes, dass aus der Sicht der Scheibentheorie Biegeschubkollapsmechanismen im Regime I nicht in einem einzigen, isolierten, senkrecht zur Balkenaxe stehenden Querschnitt lokalisiert sein können, und die $M-N-Q$-Interaktionsbeziehungen immer auch von den Bedingungen in benachbarten Querschnitten abhängen müssen. Theoretisch gibt es somit keine echte, d.h. nur querschnittsabhängige M-Q-Interaktion. Diese Probleme, auf die bereits im Abschnitt 6.3.1 hingewiesen wurde, sind schon in den Arbeiten von Drucker, Neal und anderen [11, 12, 13] im Zusammenhang mit dem Einfluss der Querkraft auf das plastische Moment von Stahlquerschnitten behandelt worden.

Die Abhängigkeit der Schubtragfähigkeit eines Querschnittes von seiner Umgebung erhält insbesondere bei der Dimensionierung Bedeutung. Diese erfolgt in der Praxis immer querschnittsweise. Die neuen Stahlbetonnormen [29, 30] fussen für die Bestimmung der Schubarmierung in wesentlichen Bereichen auf den Balkenfliessbedingungen (6.15) und (8.43). Sie erlauben insbesondere die innerhalb gewisser Grenzen freie Wahl der Neigung des Betondruckfeldes. Mit einem abrupten Wechsel dieser Neigung und damit des erforderlichen Armierungsgehaltes können aber unter Umständen die oben erwähnten Grundvoraussetzungen für die Anwendbarkeit der Balkentheorie verletzt werden. Es stellt sich also die Frage, unter welchen Voraussetzungen damit auf stabile, statisch zulässige Scheibenspannungszustände im Steg armiert wird. Grundlagen zu ihrer Beantwortung werden einerseits in den Abschnitten 9 und 10, wo die allgemeinen Spannungszustände und Geschwindigkeitsfelder der plastifizierten Stahlbetonscheibe behandelt werden und andererseits im folgenden Abschnitt, wo die physikalische Bedeutung der verallgemeinerten Verformungen (6.10), (6.23) aufgezeigt wird, erarbeitet. Die äusserst einfachen, mit den Fliessbedingungen (6.15) zu assoziierenden Scheibenmechanismen zeigen sofort anschaulich deren Gültigkeitsgrenzen auf. 
Die physikalische Bedeutung der verallgemeinerten Verformungen (6.10), insbesondere der verallgemeinerten Schiebung (6.10b), wird sofort ersichtlich, wenn die Geschwindigkeitsfelder $u(x, y), v(x, y)$ hergeleitet werden, die mit dem über den Steg des plastifizierten Querschnittes konstanten Spannungszustand im Regime I verträglich sind (Fig. 6.6).

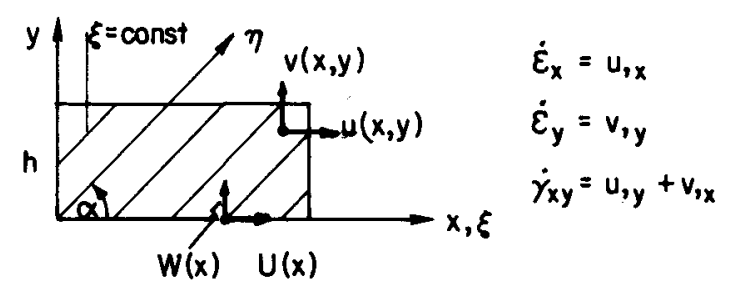

Fig. 66
Die Geschwindigkeitsfelder $u, v$ sind mit dem Regime I verträglich, wenn sie der kinematischen Bindung (6.4) bzw. (6.5) genügen. Sie sind mit einem konstanten Stegspannungszustand im Regime I verträglich, wenn die Hauptrichtungen a des Betonspannungszustandes und der Verzerrungsgeschwindigkeiten übereinstimmen, und a somit konstant ist. Dabei wird stillschweigend vorausgesetzt, dass $\alpha$ auch in einer

endlichen Umgebung des Querschnittes konstant sei, was später verifiziert wird. Mit (6.5) genügen $u$ und $v$ folglich dem linearen partiellen Differentialgleichungssystem

$\left.\begin{array}{rl}v, x+u, y+2 \cot \alpha u, x & =0, \\ v_{, y}-\cot ^{2} \alpha u, x & =0,\end{array}\right\}$

wobei Ableitungen nach $x$ und y mit ( , , bzw. ( ), bezeichnet werden. Das Differentialgleichungssystem kann auf eine partielle Differentialgleichung 2. Ordnung parabolischen Typs für u allein zurückgeführt werden. Diese geht für die koordinatentransformation

$\left.\begin{array}{l}\xi=x-y \cot \alpha, \quad \eta=y, \\ \xi_{, x}=1, \quad \xi_{, y}=-\cot \alpha, \eta_{, x}=0, \eta_{, y}=1\end{array}\right\}$

in die Normalform über. Werden die Ableitungen nach $x$ und y durch die Ableitungen nach $\xi$ und $n$ ausgedrückt, ergibt sich nach kurzer Umformung

$v_{, \xi}+\cot \alpha u, \xi+u, \eta=0$

$v, n+\cot \alpha u, n=0$

Durch Ableiten von (6.30a) nach $n$ und von (6.30b) nach $\xi$ verifiziert man sofort $u$, $n n=0$. Direkte Integration von (6.30b) und Einsetzen in (6.30a) ergibt

$\left.\begin{array}{l}v+u \cot \alpha=f(\xi), \\ u, \eta=-(v+u \cot \alpha)_{, \xi}=-f^{\prime}(\xi),\end{array}\right\}$

und somit

$u=-\eta f^{\prime}(\xi)+g(\xi)$,

$v=f(\xi)-\cot \alpha\left(g(\xi)-n f^{\prime}(\xi)\right)$. 
Die noch unbekannten Funktionen $f(\xi), g(\xi)$ sind aus den Randbedingungen z.B. für den Rand $y=0(\xi=x, n=0)$ zu bestimmen:

$\left.\begin{array}{l}u(y=0)=g(x)=U(x), \\ v(y=0)=f(x)-g(x) \cot \alpha=W(x), \\ f(x)=W(x)+U(x) \cot \alpha .\end{array}\right\}$

Der allgemeinste Ausdruck für die Geschwindigkeitsfelder in der Stegscheibe lautet somit, wenn der konstante Winkel $\alpha$ und die Geschwindigkeitskomponenten $U$, W des Randes y $=0$ vórgegeben sind,

$\left.\begin{array}{l}u=U(\xi)-\eta\left\{W^{\prime}(\xi)+U^{\prime}(\xi) \cot \alpha\right\}, \\ v=W(\xi)+n \cot \alpha\left\{W^{\prime}(\xi)+U^{\prime}(\xi) \cot \alpha\right\} .\end{array}\right\}$

Die Dehnungsgeschwindigkeiten ergeben sich zu

$\dot{\varepsilon}_{x}=\dot{\varepsilon}_{y} \tan ^{2} \alpha=-\frac{1}{2} \dot{\gamma}_{x y} \tan \alpha=u_{, x}=U^{\prime}(\xi)-n\left\{W^{\prime \prime}(\xi)+U^{\prime \prime}(\xi) \cot \alpha\right\} \geq 0$,

wobei U. W so zu wählen sind, dass im betrachteten Bereich $\dot{\varepsilon}_{x} \geq 0, \dot{\varepsilon}_{y} \geq 0$ gilt.

Die obigen Beziehungen schreiben sich natürlich einfacher in zu den Betonhauptachsen parallelen Koordinaten und Geschwindigkeitskomponenten. Man erhält sie sofort durch Nullsetzen von cota, und sie sind folglich von der form (6.36). Die vorliegende Darstellung wird vorgezogen, weil in Stahlbetonscheiben Randbedingungen praktisch immer entlang zur Armierung paralleler Ränder in zur Armierung parallelen Geschwindigkeitskomponenten zu formulieren sind.

Fig. 6.7 zeigt einige mögliche Geschwindigkeitsfelder. Dabei interessieren vor allem diejenigen mit $U(x)=0$

$\left.\begin{array}{l}u=-n W^{\prime}(\xi), \\ v=W(\xi)+n \cot \alpha W^{\prime}(\xi),\end{array}\right\}$

da die zu den Interaktionsflächen (6.15) gehörenden Mechanismen verschwindende Dehnungsgeschwindigkeiten im Zug- oder Druckflansch $(y=0)$ aufweisen, bzw. als Kombination solcher Mechanismen aufgefasst werden können. Die Bedeutung der Gleichungen (6.34) ist aus Fig. 6.7a sofort ersichtlich: Geraden $\xi=$ const,d.h. schiefe "Querschnitte" mit Neigung $\alpha$ zur Flanschachse $y=\eta=0$, bleiben auch nach der Verformung eben und für $U=0$ (Gl. (6.34b)) um $\alpha$ zur Flanschachse geneigt. Dagegen bleiben zur x-Achse senkrechte Querschnitte nur für $\alpha=90^{\circ}$, $\cot \alpha=0$ eben, wobei $(6.34)$ in den üblichen Balkenansatz

$\left.\begin{array}{l}u=u(x)-y w^{\prime}(x), \\ v=w(x) \\ \dot{\varepsilon}_{x}=u^{\prime}-y w^{n}, \dot{\gamma}_{x y}=0,\end{array}\right\}$

mit verschwindenden Schiebungsgeschwindigkeiten übergeht. 

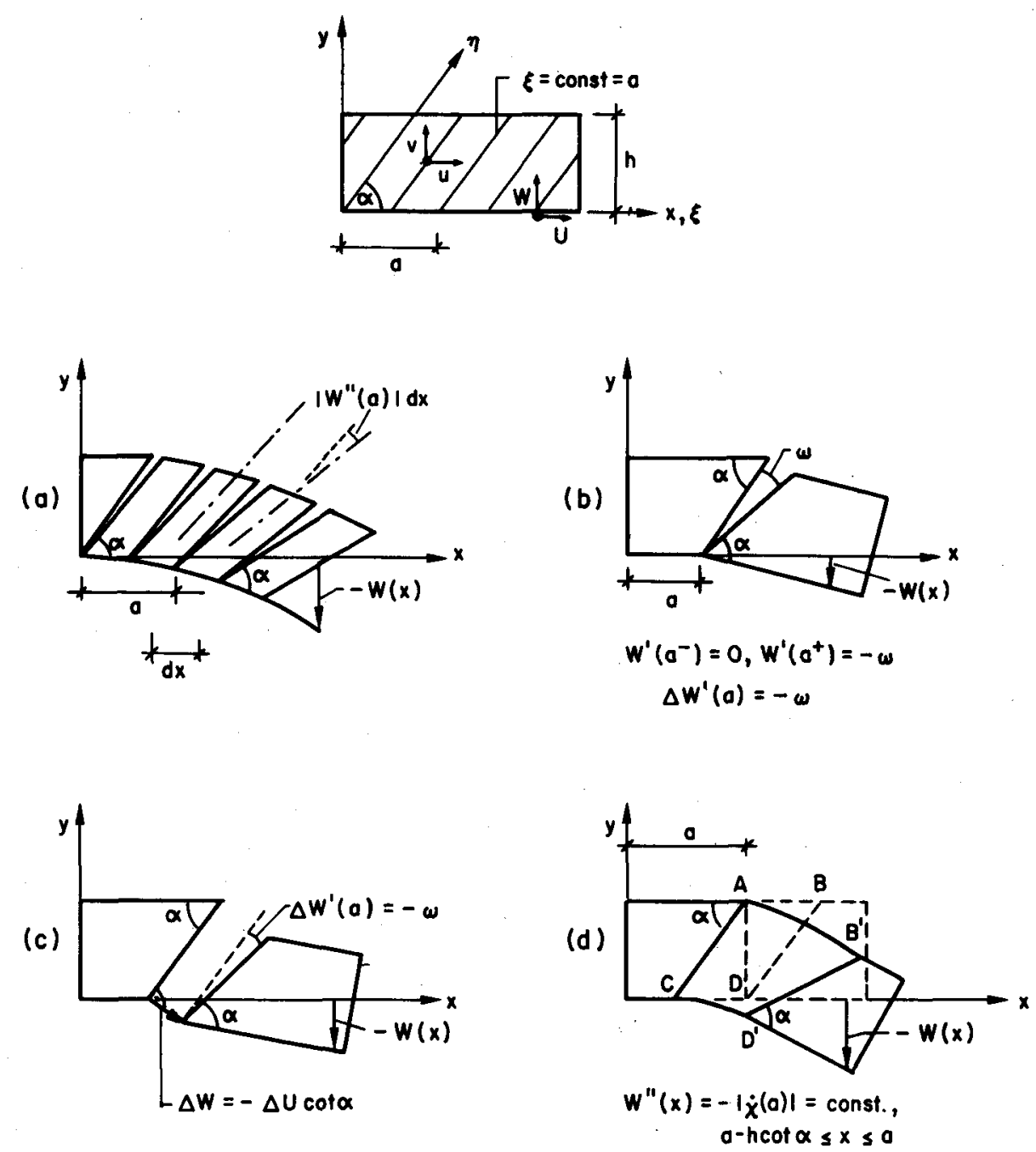

Fig. 6.7 Geschwindigkeitsfelder (6.34)

Unstetigkeiten in den ersten Ableitungen von $U, W$ in $x=$ a haben Unstetigkeiten von $u, v$ längs $\xi=a$ zur Folge (Fig. 6.7 b). Unstetigkeiten in $U$, W selbst sind nur zulässig, wenn $(W+U \cot \alpha)=(v+u \cot \alpha)$ stetig verläuft $(F i g .6 .7 \mathrm{c})$ und somit gilt

$\left.\begin{array}{l}\Delta W=-\Delta U \cot \alpha \leq 0, \\ \Delta v=-\Delta u \cot \alpha \leq 0 .\end{array}\right\}$

Solche Unstetigkeiten im Regime I sind im Abschnitt 3.3 als Kollapsrisse bezeichnet worden. Sie sind Geraden,und mit (6.37) ist wieder die senkrechte kollapsrissöffnung verifiziert.

Die Gleichungen (6.34) sind für $\alpha$ = const hergeleitet worden, gelten aber allgemeiner. Im Abschnitt 9 wird gezeigt, dass die Betondrucktrajektorien $\xi$ = const allgemeiner Spannungsfelder mit $\alpha=\alpha(x, y)$ im Regime I eine einparametrige Geradenschar $x=\xi+y \cot \alpha(\xi)$ bilden, weno das Eigengewicht der Scheibe vernachlässigt wird. Die zugehörigen verträglichen Geschwindigkeitsfelder lassen sich deshalb ebenfalls wieder durch (6.34) beschreiben, wobei $\alpha$ nun als Funktion von $\xi$ aufzufassen ist. Insbesondere bleiben auch die Kollapsrisse Geraden. Einzig die Geschwindigkeitsfelder des Regimes A (Fig. 3.2) mit verschwindenden Betonspannungen werden durch (6.34) nicht vollständig erfasst. 
Die Kenntnis der allgemeinen Form mit dem Regime I verträglicher Geschwindigkeitsfelder erlaubt es nun, noch etwas ausführlicher auf die in Abschnitt 6.3.2 getroffene Wahl der verallgemeinerten Verformungen und deren Bedeutung einzugehen. Dazu soll vorerst untersucht werden, ob mit der Verteilung (6.10), (6.23) der Verzerrungsgeschwindigkeiten in einem senkrechten Querschnitt Geschwindigkeitsfelder assoziiert werden können und wie diese aussehen.

Die Grenzen zwischen starren und im Regime I (exkl. A) fliessenden Gebieten können offensichtlich nur Geraden $\xi$ = const, d.h. Betondrucktrajektorien sein. Schiebungsgeschwindigkeiten $\dot{\gamma}_{x y} \neq 0$ in einem Querschnitt $x=a(F i g .6 .7 \mathrm{~d})$ bedingen somit wegen $\cot \alpha \neq 0$ das Fliessen des gesamten Vierecks ABCD zwischen den von den Querschnittspunkten $A$ und $D$ ausgehenden Geraden mit der in A bzw. D vorhandenen Neigung a. Da aber im plastifizierten Gebiet die Betondrucktrajektorien Geraden sein müssen, folgt aus einem gemäss (6.25) konstanten Verlauf von $\alpha$ im Querschnitt A-D die Konstanz von $\alpha$ in ganz ABCD. Damit ist die zu Beginn des Abschnittes 6.4 gemachte Annahme unter Benützung von Ergebnissen des Abschnittes 9 verifiziert.

Legt man die $x$-Achse in den Flansch, der die Neutralachse enthält $(U(x)=0)$, so folgt mit (6.35) für eine gemäss (6.10), (6.23) ebene Verteilung der Verzerrungsgeschwindigkeiten im Querschnitt $x=$ a (Fig. $6.7 d)$

$\left.\begin{array}{c}\dot{\varepsilon}_{x}=y|\dot{x}(a)|=-y W^{n}(\xi(a))=-y w^{n}(a-y \cot \alpha), \\ 0 \leq y \leq h \\ W^{\prime \prime}(x)=-|\dot{x}(a)|=\text { const, a-hoot } \alpha \leq x \leq a .\end{array}\right\}$

Die Strecke CD muss also eine konstante Krümmung aufweisen. Mit $\bar{x}=x-(a-h \operatorname{cota})$ ist das Geschwindigkeitsfeld in ABCD gegeben durch (Fig. 6.7 d)

$$
\left.\begin{array}{c}
u=|\dot{x}| \bar{x} y-|\dot{x}| y^{2} \cot \alpha, \\
v=-\frac{1}{2}|\dot{x}| \tilde{x}^{2}+\frac{1}{2}|\dot{x}| y^{2} \cot ^{2} \alpha \\
|\dot{x}|=|\dot{x}(a)|
\end{array}\right\}
$$

Wie in Abschnitt 6.3.3 schon erwähnt, wälbt also der Querschnitt $x=a$. Dies ist aber lediglich Ausdruck des Sachverhaltes, dass im Regime I der plastifizierte Querschnitt A-D für $\dot{\gamma}_{x y} \neq 0$ nicht isoliert fliessen kann, sondern dass gemäss (6.38), (6.39) das gesamte Gebiet ABCD fliessen muss, weil die Grenzen zwischen starrem und fliessendem Gebiet nicht entlang zur Balkenachse senkrechter Querschnitte verlaufen können.

Nun ist aber offensichtlich, dass der Mechanismus in Fig. 6.7 d mit der für $Q \neq 0$ von $A$ nach B stetig abnehmenden Flanschzugkraft nicht verträglich ist, wenn die Fliesskraft der Flanschlängsarmierung dem Zugkraftverlauf nicht exakt angepasst ist. Da der Gradient der Zugkraft Funktion der Querkraft ist, kann diese Verträglichkeitsbedingung höchstens für eine feste Kambination von $M$ und $Q$, nie aber für jeden Punkt der M-Q-Interaktionskurve erfüllt sein. Damit wird aber klar, dass die ebene Verteilung der Verzerrungsgeschwindigkeiten (6.10), (6.25) in einem senkrechten Querschnitt gemäss Balkentheorie lediglich formale Bedeutung hat, und dass mit den verallgemeinerten Verformungen (6.10) Scheibenmechanismen zu assoziieren sind, bei welchen die Flanschlängsarmierung nur im betrachteten Querschnitt fliesst, und die Stegscheibe sich folglich nur in einem schrägen Kollapsriss öffnet (Fig. 6.8). 


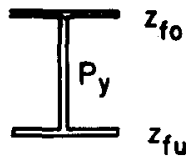

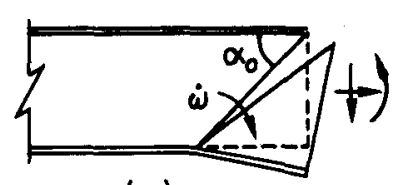

(a)

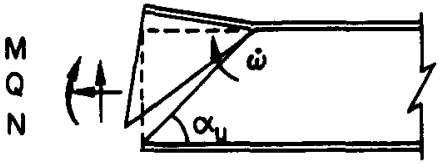

(b)

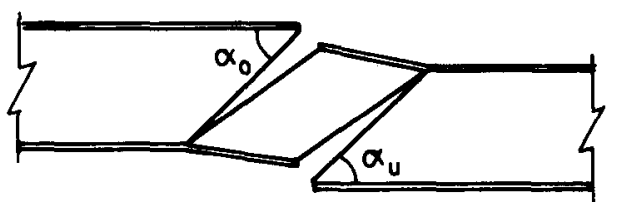

(c)

Fig: 6.8 Diskontinuierliche Biegeschubmechonismen im Regime I

Die Mechanismusmethode liefert für massgebende untere Längsarmierung (Fig. 6.8 b)

$L_{d}=\dot{w} z_{f u} h+\dot{w} \frac{1}{2} P_{y} h^{2} \cot ^{2} \alpha_{u}$,

$L_{a}=\dot{\omega} M+\dot{\omega} \frac{h}{2} N+\dot{\omega} h \cot \alpha_{u} Q$,

$M=z_{f u} h+\frac{1}{2} P_{y} h^{2} \cot ^{2} \alpha_{u}-\frac{1}{2} N h-Q h \cot \alpha_{u}$,

$\frac{\partial M}{\partial\left(\cot \alpha_{u}\right)}=P_{y} h^{2} \cot \alpha_{u}-Q h=0$,

$\cot \alpha_{u}=Q /\left(P_{y} h\right)$,

$\frac{M}{h}+\frac{N}{2}+\frac{Q^{2}}{2 P_{y}^{h}}=z_{f u}$

Entsprechend liefert der Mechanismus Fig. 6.8 a die zweite Interaktionsfläche (6.15), während auf der Schnittkurve der beiden Interaktionsflächen (Fig. 6.5 c) Mechanismen gemäss Fig. 6.8 c möglich sind. Die Identität der mit der Balkentheorie und mit den Kollapsrissmechanismen der Scheibentheorie ermittelten Interaktionsflächen zeigt, dass die durch (6.10) definierte Balkentheorie die Verzerrungsgeschwindigkeiten und die für konstante Bügelarmierung berechnete Dissipationsleistung eines schrägen Kollapsrisses formal dem senkrechten Querschnitt zuweist. Die Querschnittsbezogenheit der Mechanismen der Fig. 6.8 zeigt sich darin, dass die offenen Kollapsrissenden im betrachteten Querschnitt fixiert sind und nur deren Neigung frei ist. Dass die physikalische Bedeutung der verallgemeinerten Verformungsparameter tatsächlich der schräge Kollapsriss ist, wird besonders deutlich aus Fig. 6.g ersichtlich, die die formale Analogie in den Beziehungen zwischen den Verformungsparametern aufzeigt.
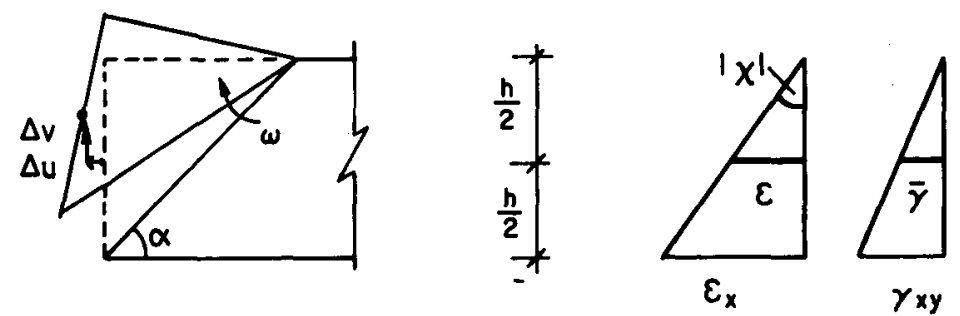

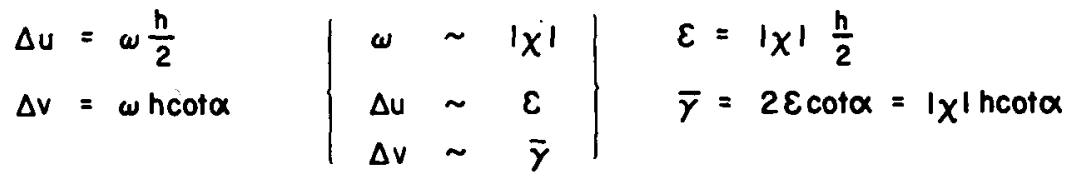

Fig. 6.9 Physikalische Bedeutung der verallgemeinerten Verformungen (6.10) 
Unter Voraussetzung der Gültigkeit der Fliessbedingung (2.7) ist die Bedeutung der "Fachwerk"Interaktionsformeln (6.15) also die folgende: Sie stellen die exakte Querschnittsinteraktion für die durch die verallgemeinerten Spannungen und Verformungen (6.10), (6.12) definierte Balkentheorie dar, wenn nachgewiesen wird, dass die Betonspannungen in Steg und Druckflansch eine obere Druckspannungsgrenze nicht überschreiten. Sie liefern obere Grenzwerte für die Traglast im Sinne der Scheibentheorie, solange die Quer- und Bügelfliesskraft über den massgebenden Kollapsriss konstant sind und dieser kinematisch zulässig ist. In diesem Fall sind mit verallgemeinerten Grössen (6.7), (6.9) abgeleitete Interaktionsformeln bestimmt auf der unsicheren Seite, da sie - weil kinematisch restriktiver - auf jeden Fall höhere Querschnittswiderstände liefern. Da man für praktische Zwecke mit über alle Bereiche des Balkens einheitlich definierten verallgemeinerten Grössen arbeiten möchte, kann nur die Wahl der verallgemeinerten Spannungen und Verformungen $(6.10)$, (6.12) in Frage kommen.

Der Querschnittsmechanismus Fig. 6.8 erlaubt auch schon einige Schlüsse bezüglich der Dimensionierungsvorschriften der Stahlbeton-Norm [29]. Diese gestattet für die Dimensionierung der Armierung die querschnittsweise freie Wahl der Druckdiagonalenneigung $3 / 5 \leq \cot \alpha \leq 5 / 3$, obschon die gemäss Fig. 6.2 a den Dimensionierungsformeln zugrunde gelegten konstanten Betonspannungszustände aus der Sicht der Scheibentheorie statisch unzulässig sind, wenn $\alpha$ von Querschnitt zu Querschnitt ändert.

Nun ist aus dem Querschnittsmechanismus Fig. 6.8 sofort ersichtlich, dass die zwei Interaktionsflächen (6.15) nur von der Bügelfliesskraft auf jeweils einer Querschnittsseite beeinflusst werden, und dass der momentan betrachtete Querschnitt zwischen den Flanschen starr bleibt und somit auch variable Spannungszustände zulässt. Offensichtlich können die Interaktionsformeln (6.15) auf rechts und links verschiedene Bügelarmierung erweitert werden bzw. bei der Dimensionierung die Neigung beider Kollapsrisse frei gewählt werden. Damit so erweiterte Interaktionsformeln auch untere Grenzwerte für die Querschnittsinteraktion (nicht für das System:) sind, müssen verträgliche, stabile, statisch zulässige Spannungszustände zwischen den zwei Kollapsrissen gefunden werden (siehe Abschnitt 10.1).

Andererseits ist ebenfalls klar, dass die bei der Dimensionierung eines Querschnittes berechnete Bügelarmierung mindestens über die Länge des massgebenden Kollapsrisses vorhanden sein muss. Dies ist bei einer querschnittsweisen Dimensionierung nicht immer automatisch gewährleistet wie Fig. 6.10 zeigt: Der eingezeichnete Kollapsriss trifft sowohl auf die schwächere Bügel- als auch auf die schwächere Längsarmierung und der betreffende Querschnitt ist somit unterdimensioniert, wenn nicht eine Ueberlappung der Armierungssprungstellen vorgeschrieben wird. Die Untersuchung allgemeiner ebener Spannungszustände der plastifizierten Stahlbetonscheibe drängt sich somit schon aus der Sicht des Schubproblems in Balken auf, um fest-bzw. sicherzustellen, dass auf stabile, statisch zulässige Scheibenspannungszustände

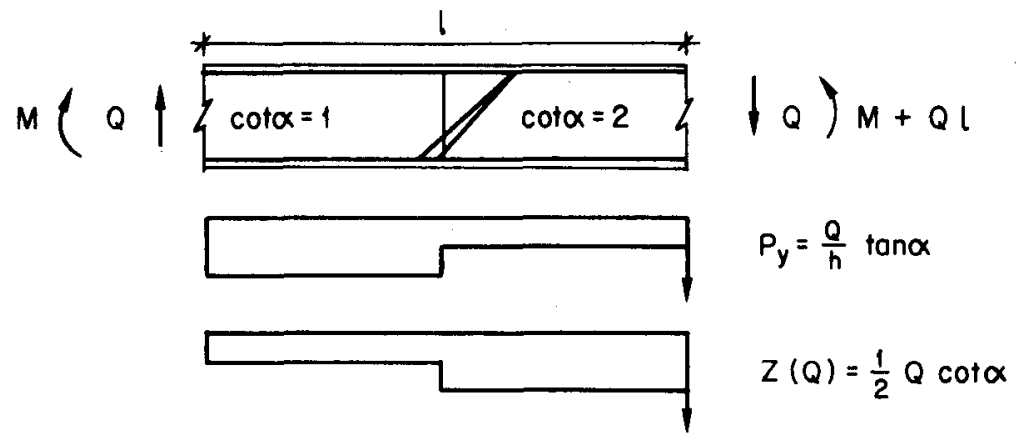

Fig. 6.10 Erforderliche Armierung infolge Querkraft bei querschnittsweiser Dimensionierung 
armiert wird.

Bis jetzt ist lediglich von der Traglast des Querschnittes im Regime I gesprochen worden als Grundlage für die Dimensionierung der Armierung. Es soll nun noch kurz auf die Traglast des Systems eingegangen werden, insbesondere auf die Frage, ob in Versuchen beobachtetes Biegeschubversagen durch die Balkentheorie des Abschnittes 6.3.2, d.h. durch Plastifizierung des massgebenden Querschnittes im Regime I allein erklärt werden kann. Dies ist offensichtlich nur dann der Fall, wenn der massgebende Querschnitt infolge zu schnell abgestufter wirksamer Querschnittshöhe, Längsarmierung (Fig. 6.11 a) und/oder Bügelarmierung (Fig. 6.10) im freien Feld liegt. Bei konstant durchgezogener Längs- und Bügelarmierung liegt der massgebende Querschnitt natürlich immer im Bereich von Einzellasten oder Auflagern (Fig: 6.11.b), und die Herleitung von (6.15) über den Mechanismus Fig. 6.8 stimmt nicht mehr, weil entweder auf dem rotierenden Dreieck die Einzellast steht oder der Kollapsriss kinematisch unzulässig ist. Die Ermittlung der Systemtraglast mit Kollapsrissmechanismen ergibt in diesen Fällen immer die volle Biegetragfähigkeit, weil mit der in (6.34) enthaltenen kinematischen Restriktion (6.4) die Betonspannungen als interne Reaktionen eingeführt werden und somit auch unendlich sein können. Ohne Einbezug des Versagens des Betons, d.h. der übrigen Fliessregimes der Fliessbedingung (2.7), kann in diesen Fällen eine Abminderung der Tragfähigkeit durch Querkraft nicht erklärt werden. Die beiden in Fig. 6.11 b dargestellten Fälle werden in Abschnitt 10.3 näher untersucht.
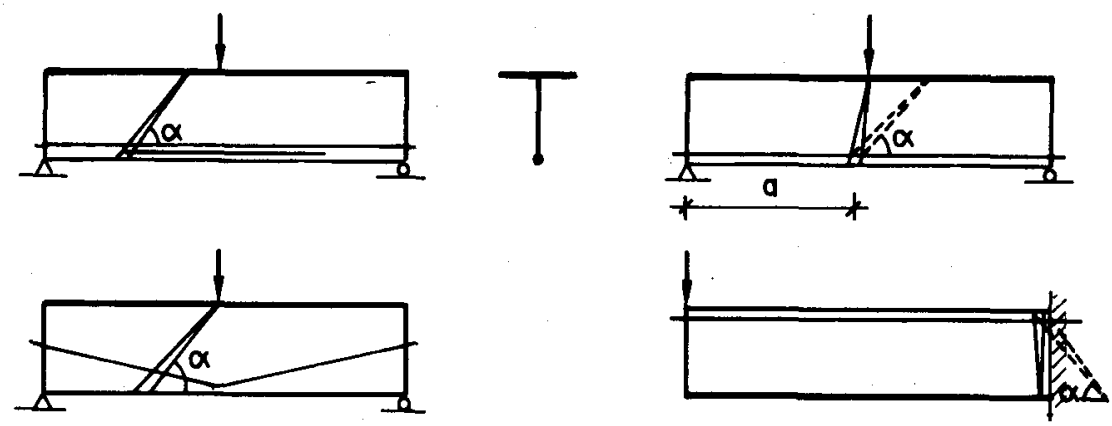

(a)

(b)

\section{Fig. 6.11 Durch Plostifizierung im Regime I erklärbares (o) und nicht erklörbares (b) Schubversogen}

\subsection{Zusammenfassung der Resultate}

In Abschnitt 6 wurde der Problemkomplex plastische Scheibentheorie - plastische Balkentheorie - Fachwerkmodell für den besonders einfach überblickbaren Fall der M-N-Q-Interaktion von I-, T- und symmetrischen a-Stahlbetonprofilen behandelt. Die Zusammenhänge bilden die Grundlage für die Behandlung der etwas komplizierteren M-N-T-Interaktion in Abschnitt $\theta$ und seien deshalb nochmals zusammengefasst.

Die Abschnitte 6.1 und 6.2 zeigen, dass die Optimalisierung bezüglich der Neigung der starren "Fachwerkdiagonalen" gleichbedeutend mit der impliziten Verwendung der Scheibenfliessbedingung ( 2.7 I) ist. Der Beton wird somit als Kontinuum eingeführt, und die Kinematik darf folglich nicht mehr am diskreten Fachwerk formuliert werden.

Aus Abschnitt 6.3 geht hervor, dass mit der "Fachwerkmodell"-Annahme eines konstanten Beton- 
spannungszustandes im Steg implizit die Definition (6.12) für die verallgemeinerten Spannungen verwendet wird. Man erhält die Interaktionsbeziehung (6.15) auch über die Dissipationsleistung des Querschnittes, wenn ihrer Berechnung die zugeordneten verallgemeinerten Verformungen (6.10) zugrundegelegt werden.

Schliesslich wird in Abschnitt 6.4 aufgezeigt, dass dem Uebergang von der Statik des diskreten Fachwerks zur Statik der Scheibe mit Fliessbedingung (2.7 I) der Uebergang von der Fachwerkkinematik zur "Kollapsrisskinematik" der Scheibe entspricht. Die Verwandtschaft mit der Fliessgelenktheorie der Stahlbetonplatten ist offensichtlich, und der Zusammenhang wird in Abschnitt 9 noch klarer. Die physikalische Deutung der verallgemeinerten Verformungen (6.10) als schräge Kollapsrisse erlaubt eine einfache Ueberprüfung der Gültigkeitsgrenzen von (6.15).

Das Fachwerk einerseits und die Kollapsrisskinematik andererseits vermitteln je ein anschauliches Bild des Kräftespiels und der Kinematik der im Regime I plastifizierten Stahlbetonscheibe.

Da die historische Entwicklung vom $45^{\circ}$-Fachwerk ausging, wurden in der "Fachwerkmodel1" Theorie die beiden grundlegenden Schritte zur Gewinnung der Interaktionsbeziehung für einen aus Scheiben zusammengesetzten Querschnitt - nämlich

a) die Wahl oder Formulierung einer Scheibenfliessbedingung,

b) die Definition verallgemeinerter Spannungen und Verformungen,

nicht explizit formuliert. Mit der hier vorgenommenen expliziten Formulierung der beiden Schritte und der Deutung der verallgemeinerten Verformungen als schräge Kollapsrisse ist eine Klärung sowohl der Stellung des "Fachwerkmodells" innerhalb der Plastizitätstheorie als auch der Grundannahmen und ihrer theoretischen Konsequenzen angestrebt warden. Erst dies ermöglicht den Vergleich mit anderen Theorien und die Beurteilung der Gültigkeitsgrenzen der Theorie.

Eine solche Standortbestimmung erscheint angesichts der in der Literatur anzutreffenden Missverständnisse nicht ungerechtfertigt. In [27] wird die Meinung vertreten, das statische Vorgehen gemäss Fachwerkmodell (Fig. 8.2 a) entspreche in Wirklichkeit der Mechanismusmethode, weil der "Skew-Bending"-Mechanismus für Biegung und Torsion tiefere Traglasten liefert. Die Klarstellung erfolgt in Abschnitt 8. Andererseits findet man auch in neuester Schubliteratur die Feststellung, die Fachwerkmodell-Lösung vernachlässige die Biegebeanspruchung der in den Flanschen eingespannten Druckdiagonalen. Solche Ueberlegungen basieren aber auf dem Konzept Makromodell "Diskretes Fachwerk" (Fig. 6.1). Sie verkennen die Tatsache, dass die Balkenfliessbedingung (6.15) nicht mehr auf der Idealisierung der Stege durch diskrete, durch Risse getrennte Betondruckdiagonalen und diskrete Bügel beruht, sondern auf der Idealisierung des Steges als Scheibe mit der Fliessbedingung (2.7 I). Nur das Fachwerk mit fester $45^{\circ}$-Diagonale und damit fester Geometrie ist ein echtes Makromodell und liefert die einfachste Linearisierung der Interaktionsbeziehungen (6.15), genau gleich wie mit Sandwich-Querschnitten linearisierte Interaktionsbeziehungen für Vollquerschnitte erhalten werden können. 
Nach der neuen Richtlinie 34 [29] der schweizerischen Stahlbetonnorm SIA 162 ist der Berechnung der Büge1- und Längsarmierung schubbeanspruchter Balken ein "Fachwerk mit" innerhalb gewisser Grenzen "frei wählbarer Diagonalenneigung" zugrunde zu legen. In Bereichen hoher Schubbeanspruchung dürfen keine zusätzlichen Querschnittswiderstände berücksichtigt werden. Der Voraussetzung nicht vorzeitig versagenden Betons wird dabei durch die Festlegung einer oberen Grenze für die nominelle Stegschubspannung Rechnung getragen. Während damit in den Bereichen hoher Schubbeanspruchung die Bemessung der Schubarmierung erstmals auf rein plastizitätstheoretischer Grundlage erfolgt, basiert die Dimensionierung der Betonabmessungen des Steges auf dem empirisch und unabhängig von der eingelegten Bügelarmierung angesetzten Wert für die obere Schubspannungsgrenze.

Die Nachrechnung von Schubversuchen mit dem Scheibenprogramm WALL [48] hat gezeigt (siehe [39]), dass die Plastizitätstheorie auch bei massgebender Betondruckfestigkeit im Steg vernünftige Resultate liefern kann. In Abschnitt 6 ist gezeigt worden, dass die Interaktionsbeziehungen (6.15) theoretisch auf den Annahmen der Balkentheorie und der Fliessbedingung (2.7 I) fussen. Es liegt deshalb nahe, zu überprüfen, ob durch den Einbezug der übrigen Fliessregimes (2.7) auch für die obere Schubspannungsgrenze ein brauchbarer plastizitätstheoretischer Ansatz aufgrund der Balkentheorie gewonnen werden kann. Schubversuche an Balken, bei welchen Versagen des Stegbetons eingetreten ist, stellen zudem zur Zeit die einzigen experimentellen Resultate dar, mit denen die Gültigkeit der im folgenden verwendeten Fliessbedingungen III bis $V$ überprüft werden kann. Die folgenden Ueberlegungen beschränken sich allerdings auf Schub infolge Querkraft, da bei Torsion durch die Verwindung zusätzliche meist nicht vernachlässigbare Querbiegespannungen in den Betondruckdiagonalen induziert werden [34]. Ebenf'alls ausgeklammert bleiben natürlich im vorliegenden Rahmen alle Aspekte der oberen Schubspannungsgrenze, die den Gebrauchszustand betreffen.

Nach dem Entstehen des vorliegenden Abschnittes ist dem Autor die Arbeit von Braestrup [26] zur Kenntnis gebracht worden. In dieser werden die gleichen Ausdrücke für die obere Schubspannungsgrenze von mit Vertikalbügeln armierten Trägern hergeleitet. Differenzen ergeben sich lediglich in der physikalischen Interpretation der Kollapsmechanismen.

\subsection{Balkentheorie}

Mit der Frage nach der oberen Grenze für die nominelle Stegschubspannung wird danach gefragt, wie weit die Schubtragfähigkeit von I-, T- oder Kastenträgern durch Zulage von Bügel- und Längsarmierung gesteigert werden kann, wenn nicht a priori nichtmassgebende Betondruckfestigkeit angenammen wird. Aus der Sicht der Balkentheorie ist also der. grösstmögliche Querkraftwiderstand zu berechnen, wenn die Fliessbedingungen des Steges für vertikale Bügel durch die Fliessfigur 2.5, für Schrägbügel durch die Fliessfigur 4.2 c gegeben sind. Der Berechnung werden wieder gemäss Fig. 6.4 idealisierte Querschnitte zugrundegelegt. Koordinatensysteme, Vorzeichenkonventionen und Bügelarmierung sind aus Fig. 7.1 ersichtlich. Die Schrägbügelrichtung $s$ entspricht der n-Richtung gemäss Abschnitt. 4.1.

Punkte maximaler Querkraft einer M-N-Q-Interaktionsfläche sind dadurch charakterisiert, dass an sie zur M-N-Ebene parallele Tangentialebenen gelegt werden können und somit verallgemeinerte Verformungsgeschwindigkeiten $\dot{\bar{\gamma}} \neq 0, \dot{\varepsilon}=\dot{x}=0$ möglich sind. Der Steg eines plasti- 

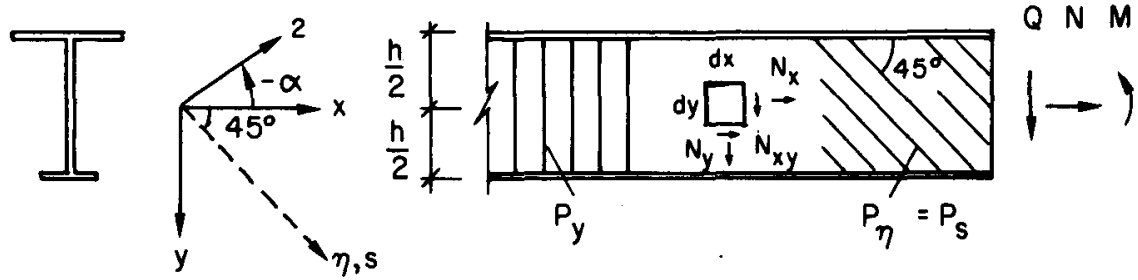

Fig. 7.1 Koordinatensysteme und Vorzeichenkonventionen

fizierten uerschnittes befindet sich also mit (6.10a)

$\dot{\varepsilon}_{x}=\dot{\varepsilon}+\dot{x} y=0$

in den Fliessregimes $V$, III oder VII (Figuren 2.5,2.6,3.2, 4.2 c), die für fehlende verteilte Längsarmierung $\left(P_{x}=0\right)$ im Steg zu Kanten degenerieren (z.B. Fig. 4.2 a).

In der Balkentheorie wird die Normalspannung quer zur Balkenaxe als nichtrelevant vernachlässigt,

$N_{y}=0$,

und als neue Fliessfigur im $N_{x}{ }^{-N}$ xy -Raum die Schnittkurve der Fliessfiguren 2.5 bzw. $4.2 \mathrm{C}$ mit der Koordinatenebene $N_{y}=0$ eingeführt. Da diese immer ausserhalb des Regimes VII verläuft, kann letzteres ausgeschlossen werden, und der Steg des plastifizierten Querschnittes befindet sich in den Regimes $V$ oder III.

Tatsächlich sind Schubversuche an I-Trägern, bei welchen Versagen des Stegbetons eingetreten ist (Schiefer Druckbruch, "web crushing", siehe Abschnitt 7.3), dadurch charakterisiert, dass die Längsarmierung die Fliessgrenze im Bruchbereich nicht erreicht hat, der Druckflansch oft sogar noch beim Bruch im Steg intakt geblieben ist und die Bügel fallweise geflossen sind $(\rightarrow$ Regime $V)$ bzw. die Fliessgrenze nicht erreicht haben $(\rightarrow$ Regime III).

Für vertikale Bügel (Fig; 2.5) schneidet die Ebene $N_{y}=0$ die Fliessfigur im Regime $V$ bzw. III, je nachdem ob $P_{y} \leq \frac{1}{2} P_{b}$ ader $P_{y} \geq \frac{1}{2} P_{b}$ gilt. Einsetzen von (7.2) in (2.7 V) bzw. (2.7 III) ergibt sofort die maximale Schubspannung innerhalb der Balkentheorie

$N_{x y}^{2}+\left(\frac{1}{2} P_{b}-P_{y}\right)^{2}=\left(\frac{1}{2} P_{b}\right)^{2} \quad$ oder

$N_{x y}= \pm \sqrt{P_{y}\left(P_{b}-P_{y}\right)}, P_{y} \leq \frac{1}{2} P_{b}$,

bzw.

$N_{x y}= \pm \frac{1}{2} P_{b} . \quad, P_{y} \geq \frac{1}{2} P_{b}$.

Unabhängig davon, ob von verallgemeinerten Spannungen (6.9) oder (6.12) ausgegangen wird, ergibt sich die grösstmögliche Querkraft für über den Steg konstante Schubspannung:

$Q= \pm h \sqrt{P_{y}\left(P_{b}-P_{y}\right)}, P_{y} \leq \frac{1}{2} P_{b}$.

$Q= \pm \frac{1}{2} \cap P_{b}$

, $P_{y} \geq \frac{1}{2} P_{b}$. 
Für die Neigung $\alpha$ der Betondrucktrajektorien folgt aus (2.1), (7.2) und Fig. 2.6 für das Regime $V$

$N_{y}=z_{y}+n_{y}=P_{y}-P_{b} \sin ^{2} \alpha=0$,

$\sin \alpha=\mp \sqrt{P_{y} / P_{b}}, P_{y} \leq \frac{1}{2} P_{b}$,

und für das Regime III

$n_{y}=-\frac{1}{2} P_{b}=-P_{b} \sin ^{2} \alpha$,

$\alpha=\mp 45^{\circ}, \quad P_{y} \geq \frac{1}{2} P_{b}$.

Natürlich lässt sich das diesen Beziehungen zugrundeliegende Kräftespiel (nicht aber die zugehörige Kinematik) wieder als "Fachwerk" deuten, dessen Diagonalenneigung (im Regime V) aus der bedingung bestimmt wird, dass Bügel und Betondruckdiagonalen "fliessen". Voraussetzung für das Erreichen des Querkraftwiderstandes (7.4) ist, dass die Flanschen bzw. deren Armierung und die Steglängsarmierung die Kräfte

$Z_{u, 0}= \pm \frac{M}{h}+\frac{N}{2}+\left|\frac{Q}{2} \cot \alpha\right|$

aufnehmen können.

Interessante Anhaltspunkte für die. Brauchbarkeit der verwendeten Fliessbedingungen bzw. der Plastizitätstheorie überhaupt lassen sich aus dem Vergleich des Schubbruchverhaltens von Trägern mit Vertikalbügeln und Schrägbügeln gewinnen, da letztere wesentlich kleinere Betondruckspannungen zur Folge haben. Die entsprechenden Beziehungen werden deshalb auch für Schrägbügel hergeleitet (Fig. 7.1).

Aus Fig. 4.2 c liest man ab, dass die Ebene $N_{y}=0$ die Fliessfigur im Regime $V$ bzw. III schneidet, je nachdem ob $P_{n}=P_{s} \leq\left(1 \pm \frac{1}{2} \sqrt{2}\right) P_{b}$ oder $P_{s} \geq\left(1 \pm \frac{1}{2} \sqrt{2}\right) P_{b}$ gilt. Das negative Vorzeichen gilt dabei für "falsch" geneigte Schrägbügel bzw. für negatives $N_{x y}$ oder $Q$. $P_{s}=P_{\eta}$ bezeichnet die Bügelfliesskraft pro Längeneinheit senkrecht zur Armierungsrichtung.

Im Regime $V$ gilt gemäss Abschnitt 4.1

$z_{n}=P_{n}=P_{s}$, bzw. mit $(4 \cdot 3)$

$z_{y}=z_{x y}=\frac{1}{2} P_{s}$

$n_{1}=0, n_{2}=-P_{b}, \quad b z w$.

$n_{x y}^{2}=n_{x} n_{y}$

$n_{x}+n_{y}=-P_{b}$ 
Berücksichtigung von (7.2) und (7.7) in (2.1) ergibt

$N_{y}=n_{y}+z_{y}=n_{y}+\frac{1}{2} P_{s}=0$

$N_{x y}=n_{x y}+z_{x y}=n_{x y}+\frac{1}{2} P_{s}$.

Durch Einsetzen von (7.9) bis (7.11) in (7.8) folgt für die maximale Schubspannung innerhalb der Balkentheorie

$$
\left.\begin{array}{c}
\left(N_{x y}-\frac{1}{2} P_{s}\right)^{2}=-\frac{1}{2} P_{s}\left(-P_{b}+\frac{1}{2} P_{s}\right), \\
N_{x y}=\frac{1}{2} P_{s} \pm \sqrt{\frac{1}{2} P_{s}\left(P_{b}-\frac{1}{2} P_{s}\right)}, \\
P_{s} \leq\left(1 \pm \frac{1}{2} \sqrt{2}\right) P_{b},
\end{array}\right\}
$$

solange die Schrägbügel die Fliessgrenze erreichen (Regime V Fig. 4.2 c). Bei gegebenem $P_{b}$ wird (7.12a) maximal bzw. minimal für $P_{S}=\left(1 \pm \frac{1}{2} \sqrt{2}\right) P_{b}$,

$$
\begin{aligned}
& N_{x y}=\frac{1}{2} P_{b}(1 \pm \sqrt{2}), \\
& P_{s} \geq\left(1 \pm \frac{1}{2} \sqrt{2}\right) P_{b},
\end{aligned}
$$

und kann nicht mehr weiter gesteigert werden, da für $P_{s}>(1 \pm \sqrt{2}) P_{b}$ Versagen im Regime III eintritt und die Schrägbügel die Fliessgrenze nicht mehr erreichen. Dabei ist der Maximalwert (7.12b) lediglich von theoretischer Bedeutung, da der entsprechende Armierungsgehalt (im Gegensatz zu Vertikalbügeln) in der Praxis kaum realisierbar ist. Multiplikation von (7.12) mit h ergibt wieder die Maximal-bzw. Minimalwerte für die Querkraft.

Für die Neigung der Betondrucktrajektorien im Regime $V$ ergibt sich

$$
\begin{aligned}
& N_{y}=z_{y}+n_{y}=\frac{1}{2} P_{s}-P_{b} \sin ^{2} \alpha=0 \\
& \sin \alpha=\mp \sqrt{\frac{1}{2} P_{s} / P_{b}}, P_{s} \leq\left(1 \pm \frac{1}{2} \sqrt{2}\right) P_{b} .
\end{aligned}
$$

Im Regime III ist die Orucktrajektorienneigung unabhängig vom Bügelgehalt, und durch Auswerten von (7.13a) für den Grenzarmierungsgehalt folgt

$$
\left.\begin{array}{l}
\alpha=-67.5^{\circ} \text { bzw. }+22 \cdot 5^{\circ}, \\
P_{S} \geq\left(1 \pm \frac{1}{2} \sqrt{2}\right) P_{b} .
\end{array}\right\}
$$

Die Grenzneigungen (7.13b), (7.5b) sind kinematisch sofort evident (siehe auch Abschnitt 7.2): Die maximale Schubspannung wird für nicht massgebende Längs- und Bügelarmierung erreicht. Für verschwindende Dehnungsgeschwindigkeiten in beiden Armierungsrichtungen müssen aber die Hauptrichtungen der Verzerrungsgeschwindigkeiten und der Betonspannungen mit den Winkelhalbierenden der Armierungsrichtungen zusammenfallen.

Das zweite Vorzeichen in (7.12), (7.13) bezieht sich auf den Minimalwert für $N_{x y}$ bzw. auf in der "falschen" Richtung eingelegte Schrägbügel. Es wurde lediglich mitgeführt, um die Bedeutung der Grenzneigung klarer hervortreten zu lassen. Die entsprechenden Werte dürften jedoch 
kaum mehr einen Bezug zur Realität haben, da zu erwarten ist, dass bei Trägern mit praktisch parallel zur Anrissrichtung verlegten Bügeln die vorausgesetzten Spannungsumlagerungen nicht möglich sind, und wie bei Trägern ohne Schubarmierung ein Sprödbruch eintritt.

Voraussetzung für das Erreichen des Querkraftwiderstandes (7.12) ist, dass die Flanschen bzw. deren Armierung die Kräfte

$Z_{u, 0}= \pm \frac{M}{h}+\frac{N}{2}-\frac{h}{2} N_{x}$

aufnehmen können. $N_{x}$ beträgt dabei für fehlende Steglängsarmierung $P_{x}=0$

$N_{x}=n_{x}+z_{x}=-P_{b}+P_{5}$.

Wegen der Identität der Fliessregimes III und $V$ in Fig. 2.5 und Fig. 4.4 gelten die Beziehungen (7.3), (7.4) für vertikale Bügel ebenfalls, wenn die wirklichkeitsnahere Betonfliessfigur 4.3 b zugrundegelegt wird. Dagegen zeigt der Vergleich von Fig. 4.2 c und Fig. 4.6, dass dies auf die Beziehungen (7.12) für Schrägbügel nur zutrifft, wenn

$P_{\eta}=P_{s} \leq P_{b}$

gilt. Für in der Praxis vorkommende Bügelarmierungsgehalte ist (7.15) immer erfüllt.

Für den Vergleich mit Versuchsresultaten (Abschnitt 7.3) sollen (7.3) bzw. (7.4) und (7.12) noch in dimensionsloser Form geschrieben werden. Mit $b_{0}$ wird die Stegbreite, mit $k b_{0} d i e$ mitwirkende Stegbreite bezeichnet. Mit der bezogenen nominellen Stegschubspannung $\bar{\tau}$,

$\frac{Q / h}{P_{b}}=\frac{N_{x y}}{P_{b}}=\frac{\tau b_{o}}{\beta_{p} K b_{0}}=\frac{\tau}{\kappa \beta_{p}}=\bar{\tau}$,

und dem bezogenen Bügelarmierungsgehalt $\bar{\mu}$ (Fig. 2.2),

$\frac{P_{y}}{P_{b}}=\frac{F_{y} \sigma_{f}}{t_{y} \kappa b_{o} \beta_{p}}=\mu \frac{\sigma_{f}}{\kappa \beta_{p}}=\bar{\mu}$,

ergibt sich für Vertikalbügel

$\bar{\tau}=\sqrt{\bar{\mu}(1-\bar{\mu})} \leq \frac{1}{2}$

und entsprechend für Schrägbügel

$\bar{\mu}=P_{s} / P_{b}$,

$\bar{\tau}=\frac{\bar{\mu}}{2}+\sqrt{\frac{\bar{\mu}}{2}\left(1-\frac{\bar{\mu}}{2}\right)} \leq \frac{1}{2}(1+\sqrt{2})$.

Mit (7.18), (7.19) ist auch die geeignete Form für den Vergleich des plastizitätstheoretischen Ansatzes mit den in den Normen festgelegten Schubspannungsgrenzen gewannen.

Innerhalb der Balkentheorie, die nur von der Fliessfigur im $N_{x}{ }^{-N_{x y}}$-Raum ausgeht und sich somit um eventuelle Querdehnungen nicht kümmert, ist der Mechanismus gemäss (7.1) sehr einfach: eine reine über den Querschnitt konstante Schiebung. (7.4) ergänzt somit die Interaktionsfiguren 6.5 b,c durch eine horizontale Gerade bzw. Ebene im Abstand (7.4) von der N-Axe bzw. $M-N$-Ebene. Der hintere Abschluss der Interaktionsfigur 6.5 folgt aus hier nicht untersuchten 
Mechanismen mit $\dot{\varepsilon}_{x} \leq 0$.

Betrachtet man nun jedoch wieder die Scheibenfliessfigur, so bedingen in den Regimes III und $\checkmark$ der Fliessfiguren 2.5, 4.2 c plastische Schiebungsgeschwindigkeiten im allgemeinen auch plastische Querdehnungsgeschwindigkeiten $\dot{\varepsilon}_{y}$. Auch hier ist deshalb das Fliessen eines isolierten Querschnittes ohne Verletzung von Scheibenintegrabilitätsbedingungen im allgemeinen nicht möglich. Wie in Abschnitt 6 sollen daher im nächsten Abschnitt wieder mit den hier betrachteten Spannungszuständen verträgliche, kinematisch zulässige Geschwindigkeitsfelder für die Stegscheibe hergeleitet werden. Diese liefern wieder eine anschauliche physikalische bzw. scheibentheoretische Deutung der verallgemeinerten Verformung $\bar{\gamma}$ und erlauben auch den qualitativen Vergleich mit in Versuchen beobachteten Schubbruchmechanismen.

\subsection{Kinematisch zulässige, verträgliche Geschwindigkeitsfelder der Stegscheibe}

Die Geschwindigkeitsfelder $u, v(F i g .7 .2$ a) sind mit den Regimes V, III (Fig. 2.5 bzw. 3.2 , Fig. 4.2 c) verträglich, wenn sie der kinematischen Bindung

$\dot{\varepsilon}_{x}=u, x=0$,

$\dot{\varepsilon}_{y} \geq 0$ bzw. $\dot{\varepsilon}_{s} \geq 0$

genügen. Sie sind mit dem konstanten Stegspannungszustand gemäss Abschnitt 7.1 verträglich, wenn zusätzlich die Hauptrichtungen $\alpha$ der Dehnungsgeschwindigkeiten

$\tan 2 \alpha=\frac{-\dot{\gamma}_{x y}}{\dot{\varepsilon}_{y}}=\frac{-v, x-u, y}{v, y}$

mit denjenigen des Spannungszustandes im Beton übereinstimmen und a somit über den Querschnitt konstant ist. Das allgemeine Differentialgleichungssystem der in den Regimes $V$, III, VII fliessenden Scheibe (Abschnitt 9 ) zeigt wieder, dass damit a auch in einer endlichen Umgebung des Querschnittes konstant sein muss. Somit genügen u und $v$ dem folgenden linearen partiellen Differentialgleichungssystem

$\left.\begin{array}{rl}v, x+u, y+v, y \tan 2 \alpha & =0, \\ u, x & =0 .\end{array}\right\}$

Dieses kann auf eine partielle Differentialgleichung 2. Ordnung hyperbolischen Typs für $v$ allein zurückgeführt werden, die für die koordinatentransformation

$\xi \quad=x-y \cot 2 \alpha, n=y$,

$\xi_{, x}=1, \xi_{, y}=-\cot 2 \alpha, \eta_{, x}=0, \eta_{, y}=1$,

in die Normalform übergeht. Dabei bezeichnet nun $n$ nicht mehr die Schrägbügelrichtung, sondern eine der charakteristischen Richtungen. Ausdrücken der Ableitungen nach $x, y$ durch solche nach $\xi$, $n$ ergibt

$\begin{aligned} v, n+u, n \cot 2 \alpha & =0, \\ u, \xi & =0 .\end{aligned}$ 
(a)

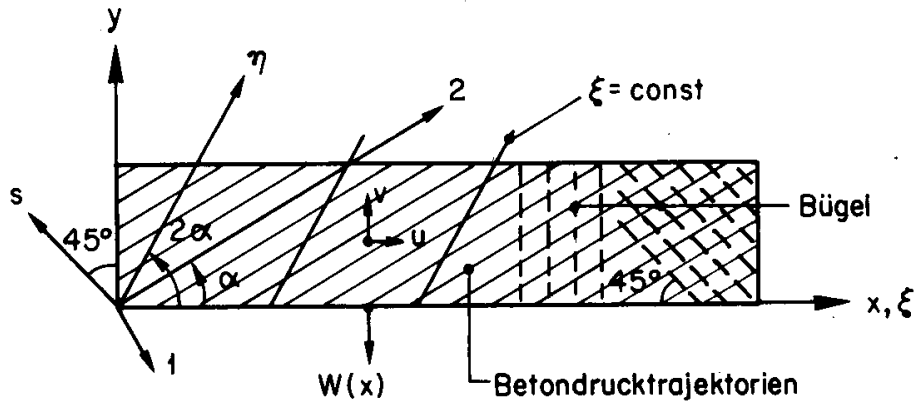

(b)

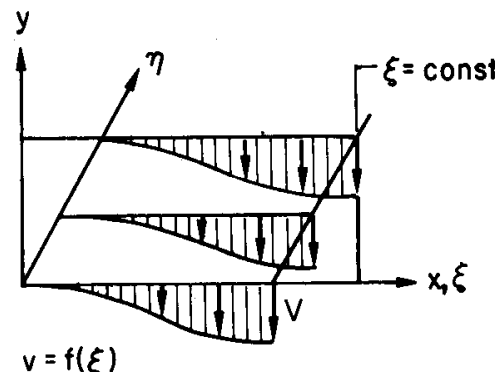

(d)

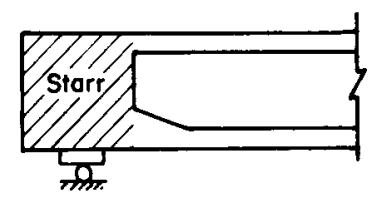

(f)

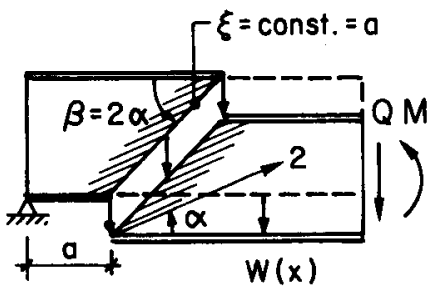

(h)

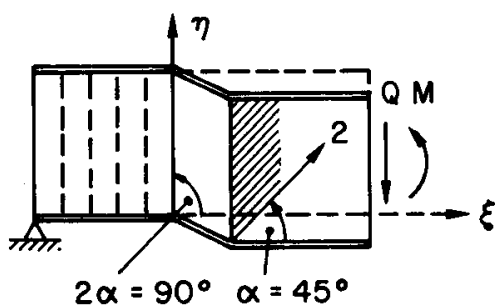

(c)

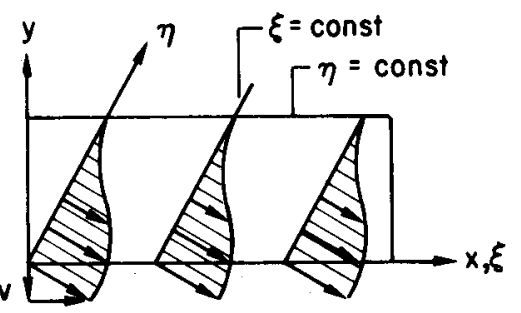

(e)

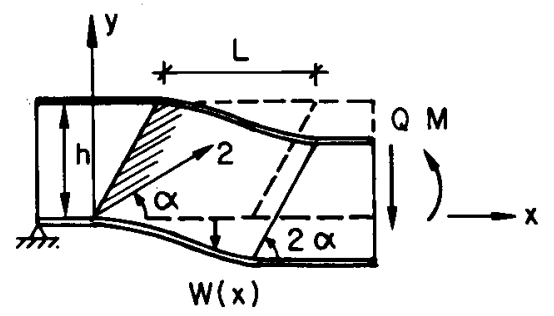

(g)

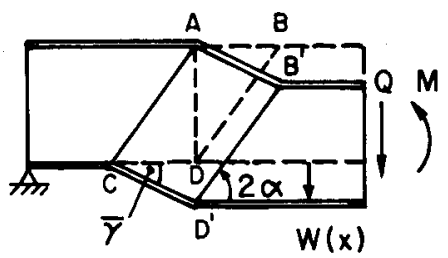

(i)

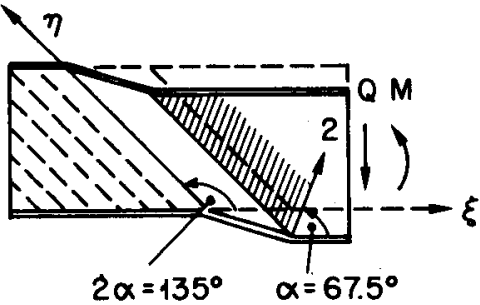

Fig. 7.2 Geschwindigkeitsfelder (7.26), (7.28) 
Durch Ableiten der zwei Gleichungen (7.24) nach $\xi$ bzw. $n$ verifiziert man sofort $v$, $\xi n=0$. Die Charakteristiken bilden zwei Geradenscharen $\xi=$ const, $\eta=$ const mit Neigung $2 \alpha$ bzw. 0 . Direkte Integration van (7.24) ergibt

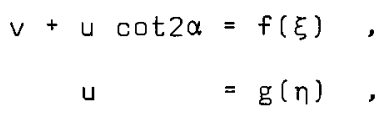

\}

und somit

$u=g(n)$,

$v=f(\xi)-g(\eta) \cot 2 \alpha$.

Mögliche Geschwindigkeitsfelder infolge $f$ sind in Fig. 7.2 b, solche infolge g in Fig. 7.2 c dargestellt. Die noch unbekannten Funktionen $f(\xi), g(n)$ sind aus den Randbedingungen zu bestimmen. Da Träger mit schlanken Stegen im Auflagerbereich immer durch einen Endblock verstärkt sind, der als starr angenommen werden darf (Fig. 7.2 d), kann u nur Iinear in y = $\eta$ sein. Weil sich aber die Geschwindigkeitsfelder infolge linearem $f$ bzw. g nur um eine Starrkörperrotation unterscheiden, kann g ohne Verlust von Allgemeinheit null gesetzt werden. Bezeichnet $W(x)$ die nach unten positive Durchbiegung des Randes $y=0$, folgt sofort

$v(y=0)=f(x)=-W(x)$,

$u=0$

\}

Geraden $\xi=x-y \cot 2 \alpha=$ const sind Drte gleicher Durchbiegung (Fig. 7.2 b). Die Biegelinie W( $x$ ) kann bis auf die Bedingung (7.31) beliebig vorgeschrieben werden (Fig. 7.2 e), insbesondere kann $W$ auch unstetig verlaufen. Dabei hat eine Unstetigkeit von $W$ in $x=$ a Unstetigkeiten von $v$ entlang $\xi=x-y \cot 2 \alpha=a$ zur Folge (Fig. 7.2 f). Solche Unstetigkeiten sind in Abschnitt 3.3 als Gleitlinien bezeichnet worden. Ihre Neigung $\beta=2 \alpha$ verifiziert man sofort auch mit $(3.18)$ und $\theta=0(\mathrm{Fig} \cdot 3.5)$.

Die Bedeutung der verallgemeinerten Verformung $\bar{\gamma}$ in der Scheibentheorie geht aus Fig. 7.2 g hervor. Diese zeigt ebenfalis wieder, dass die in der Balkentheorie angenommene plastische Schiebung in einem isolierten Querschnitt ausser im Spezialfall $2 \alpha=90^{\circ}$ (Fig. $7.2 \mathrm{~h}$ ) $\mathrm{nicht}$ möglich ist, weil das Fliessen des Querschnittes A-D das Fliessen des gesamten Vierecks ABCD bedingt, sobald die Querdehnungen berücksichtigt werden.

Für die Schiebungsgeschwindigkeiten folgt

$\dot{\gamma}_{x y}=u, y+v, x=-W^{\prime}(\xi)$

Die Dehnungsgeschwindigkeiten $\dot{\varepsilon}_{2}$ in der Hauptrichtung $\alpha, \dot{\varepsilon}_{y}$ in den Vertikalbügeln und $\dot{\varepsilon}_{\text {s }}$ in den Schrägbügeln ergeben sich zu

$\dot{\varepsilon}_{2}=\frac{1}{2} \dot{\gamma}_{x y} \tan \alpha=-\frac{1}{2} w^{\prime}(\xi) \tan \alpha$,

$\dot{\varepsilon}_{y}=-\dot{\gamma}_{x y} \cot 2 \alpha=w^{\prime}(\xi) \cot 2 \alpha$,

$\dot{\varepsilon}_{s}=-\frac{1}{2} \dot{\gamma}_{x y}(1+\cot 2 \alpha)=\frac{1}{2} W^{\prime}(\xi)(1+\cot 2 \alpha)$. 
Die Richtung $\alpha$ ist wie vorausgesetzt die Richtung der Betondrucktrajektorien, wenn $\dot{\varepsilon}_{2} k l e i-$ nere Hauptdehnungsgeschwindigkeit ist, also $\dot{\varepsilon}_{2} \leq 0$ gilt. Die einzige Restriktion für $W$ lautet damit

$\left.\begin{array}{l}W^{\prime}(\xi) \geq 0, \text { für } 0^{\circ} \leq \alpha \leq 90^{\circ}, \\ W^{\prime}(\xi) \leq 0, \text { für }-90^{\circ} \leq \alpha \leq 0^{\circ} .\end{array}\right\}$

Aus $(7.20$ b) ergeben sich schliesslich die Gültigkeitsgrenzen für $\alpha$. Für vertikale Bügel folgt mit $(7.30 b)$

$|2 \alpha| \leq 90^{\circ}, \quad|\alpha| \leq 45^{\circ}$,

während sich für Schrägbügel mit (7.30c)

$\cot 2 \alpha \geq-1$ für $\alpha \geq 0, \quad \cot 2 \alpha \leq-1$ für $\alpha \leq 0$,

$-45^{\circ} \leq 2 \alpha \leq 135^{\circ},-22.5^{\circ} \leq \alpha \leq 67.5^{\circ}$,

ergibt. Die Grenzen stimmen mit denjenigen in Abschnitt 7.1 überein, und damit sind zu allen dort betrachteten Spannungszuständen verträgliche, kinematisch zulässige Geschwindigkeitsfelder gefunden. Dabei ist zu beachten, dass gegenüber Abschnitt 7.1 das Vorzeichen von $\alpha$ gewechselt hat, weil das Koordinatensystem in Fig. 7.1 gemäss den Konventionen der Balkentheorie, in Fig. 7.2 dagegen gemäss denjenigen der Scheibentheorie eingeführt worden ist.

Für die Grenzneigungen (7.32), (7.33) werden die Bügeldehnungsgeschwindigkeiten null, und die Stegscheibe fliesst im Regime III (Fig. $2.5,4.2 \mathrm{c}$ ). Da das Koordinatennetz $\xi=$ const, $\eta=$ const in den Richtungen verschwindender Dehnungsgeschwindigkeiten verläuft, muss dieses mit den Armierungsrichtungen zusammenfallen. Entsprechende Mechanismen sind für Vertikalbügel in Fig. 7.2 h, für Schrägbügel in Fig. 7.2 i dargestellt. Sobald solche Mechanismen massgebend werden, erhöht offensichtlich zusätzliche Armierung in den Richtungen $\xi$ und $n$ den Querkraftswiderstand nicht mehr.

Da die betrachteten Spannungszustände und Mechanismen verträglich sind, folgen die in Abschnitt 7.1 berechneten Werte für $\alpha$ und $Q$ auch direkt mit der Mechanismusmethode. Die äussere Leistung beträgt (Fig. 7.2 e)

$L_{a}=Q(W(L)-W(0))$

Mit (7.30) folgt für die Dissipationsleistung

$L_{d}=\int_{F}\left(\dot{\varepsilon}_{y} P_{y}-\dot{\varepsilon}_{2} P_{b}\right) d F=\left(P_{y} \cot 2 \alpha+\frac{1}{2} P_{b} \tan \alpha\right) \int_{F} W^{\prime} d F$

für Vertikalbügel, und

$L_{d}=\int_{F}\left(\dot{\varepsilon}_{s} P_{s}-\dot{\varepsilon}_{2} P_{b}\right) d F=\left(\frac{1}{2} P_{s}(1+\cot 2 \alpha)+\frac{1}{2} P_{b} \tan \alpha\right) \int_{F} W^{\prime} d F$

für Schrägbügel. Mit (7.35)

$\int_{F} W^{\prime}(\xi) d F=\int_{F} W^{\prime}(\xi) d \xi d \eta=h(W(L)-W(0))$ 
ergibt sich durch Gleichsetzen von (7.33) und (7.34)

$Q=h\left(P_{y} \cot 2 \alpha+\frac{1}{2} P_{b} \tan \alpha\right)$,

$Q=h\left(\frac{1}{2} P_{s}(1+\cot 2 \alpha)+\frac{1}{2} P_{b} \tan \alpha\right)$.

Durch Minimieren bezüglich a erhält man für Vertikalbügel wieder

$$
\left.\begin{array}{rl}
\frac{\partial Q}{\partial \alpha}=0= & P_{y} \frac{-2}{\sin ^{2} 2 \alpha}+\frac{1}{2} P_{b} \frac{1}{\cos ^{2} \alpha}, \\
\sin \alpha & = \pm \sqrt{P_{y} / P_{b}} \\
Q & = \pm h \sqrt{P_{y}\left(P_{b}-P_{y}\right)}
\end{array}\right\}
$$

und der Vergleich von (7.36a) mit (7.36b) liefert sofort die entsprechenden Beziehungen für Schrägbügel

$$
\left.\begin{array}{l}
\sin \alpha= \pm \sqrt{\frac{1}{2} P_{s} / P_{b}} \\
Q=h\left(\frac{1}{2} P_{s} \pm \sqrt{\frac{1}{2} P_{s}\left(P_{b}-\frac{1}{2} P_{s}\right)}\right)
\end{array}\right\}
$$

In Abschnitt 9 wird gezeigt, dass die allgemeinen Geschwindigkeitsfelder in den Regimes $V$, VII (und analog in IV, VI) ebenfalls durch (7.26) dargestellt werden, wobei nun aber die Charakteristikenschar $\xi$ = const nicht mehr durch parallele, sondern durch fächerartige Geraden gebildet wird, also ein aus den Randbedingungen zu ermittelnder Zusammenhang $\alpha=f(\xi)$ besteht.

Im Hinblick auf die Besprechung des "Skew Bending"-Mechanismus in Abschnitt 8 muss wieder darauf hingewiesen werden, dass nichtsenkrechte Sprünge entlang Unstetigkeitslinien (Fig. 7.2 f) immer Stauchen des Betons bedeuten, solange mit den Fliessfiguren 2.1, 4.3 b für den Beton gearbeitet wird. Nichtsenkrechte Sprünge ohne Betonstauchen sind nur möglich, wenn die Neigung der Betondrucktrajektorien bezüglich der Armierungsrichtungen begrenzt wird und mit durch entsprechende Ebenen abgeschnittenen Betonfliessfiguren gearbeitet wird. Die zugehörige Unstetigkeitslinie hat aber dann eine bezüglich der Armierung fest fixierte Richtung.

Braestrup [26] geht bei der Herleitung von (7.18) mit der Mechanismusmethode vom unstetigen Mechanismus Fig. $7.2 \mathrm{f}$ aus und interpretiert die Unstetigkeitslinie als Riss. Damit entsteht der Eindruck, dass ein Kollapsmechanismus entlang einem vor dem Bruch beobachtbaren Schubriss behandelt wird. Die Versuchsresultate des Trägers 11 [40], der in Abschnitt 7.3 näher behandelt wird, sprechen jedoch gegen diese Interpretation.

Beim Versuchsträger T1 haben weder die Längs- noch die Bügelarmierung die Fliessgrenze erreicht, und der Träger versagte im Regime III. Im massgebenden Schubbereich waren bis zum Bruch ausschliesslich ungefähr $45^{\circ}$ geneigte Risse zu beobachten. Sie folgen ziemlich genau den theoretischen Betondrucktrajektorien, die sowohl im homagenen Zustand als auch beim Kollaps eine theoretische Neigung von $45^{\circ}$ aufweisen. Die Richtung $n$ möglicher Unstetigkeitslinien im Regime III (vertikale Bügel) ist dagegen gemäss Fig. $7.2 \mathrm{~h}$ durch $\beta=2 \alpha=90^{\circ}$ gegeben. Die theoretische Unstetigkeitsinie ist also gegenüber den vor dem Bruch beobachteten Rissen um $45^{\circ}$ geneigt. Aus diesem Grunde werden in Abschnitt 3.3 einerseits für die idealisierte Stahlbetonscheibe ohne Betonzugfestigkeit die theoretischen Begriffe (Fig. 3.8) 
"Kollapsriss" $(\beta=\alpha)$ und "Gleitlinie" $(\beta \neq \alpha)$ und andererseits die wirklichen physikalischen Risse unterschieden. Die wirklichen Risse scheinen - mit durch die partiell immer vorhandenen Betonzugspannungen bedingter Verzögerung - den theoretischen, sich entsprechend den Spannungsumlagerungen vom homogenen, über den gerissenen zum Kollaps-Zustand sich ändernden Richtung der Betondrucktrajektorien zu folgen [16, 36]. Zur Veranschaulichung sei das Verhalten von quadratischen Betonscheiben im einaxialen Druckversuch [32] herangezogen. Zuerst bilden sich feine Mikrorisse parallel zur Druckkraft. Das endgültige Versagen tritt dann entlang einer zur Druckkraft ca. $30^{\circ}$ geneigten Gleitebene auf, wie es auch entsprechend der zweiaxialen Fliessfigur 4.3 a zu erwarten ist. Schubrisse und Kollapsmechanismus Fig. $7.2 \mathrm{f}$ der Stahlbetonscheibe stehen in analoger Beziehung wie Mikrorisse und Gleitebenenmechanismus der Betonprobekörper.

7.3 Vergleich mit Versuchsresultaten und Normen

Die Schubbrucharten, auf die sich die Theorie des Abschnittes 7 bezieht, werden in der Literatur recht verschieden bezeichnet: schiefer Druckbruch, Schubdruckbruch, Stauchungs- bzw. Stauchungs-Schiebungsbruch, web crushing. Allen gemeinsam ist, dass der Stegbeton versagt, und die Hauptlängsarmierung die Fliessdehnung nicht erreicht, während die Bügel fallweise fliessen bzw. nicht fliessen. Sie treten ausschliesslich an I-, T- und Kastenträgern mit schlanken Stegen und starker Längsarmierung (schlaff oder vorgespannt) auf. In [37, 38] sind die Schubbrüche nach den beim Bruch erreichten Dehnungen in der Hauptlängsarmierung $\varepsilon_{L}$ und in den Bügeln $\varepsilon_{B}$ klassifiziert. Jedem Begriff lässt sich somit ein Fliessregime der Fliessfiguren $2.5,4.2 \mathrm{c}, 4.4$ zuordnen (Fig. 7.3).

\begin{tabular}{|c|c|l||c|c|c|c|}
\hline \multicolumn{2}{|c||}{ Versuch } & \multicolumn{4}{c|}{ Idealplastische Theorie } \\
\hline$\varepsilon_{L}$ & $\varepsilon_{B}$ & Klassifikation & $\dot{\varepsilon}_{x}$ & $\dot{\varepsilon}_{y}$ & $\dot{\varepsilon}_{2}$ & Fliessregime \\
\hline$>\varepsilon_{f}$ & $<\varepsilon_{f}$ & meist Biegebruch & $>0$ & 0 & 0 & $A-0$ \\
\hline$>\varepsilon_{f}$ & $>\varepsilon_{f}$ & Biegeschubbruch (BS) & $>0$ & $>0$ & 0 & I \\
\hline$<\varepsilon_{f}$ & $>\varepsilon_{f}$ & $\begin{array}{l}\text { Stauchungsschiebungs- } \\
\text { bruch (SS) }\end{array}$ & 0 & $>0$ & $<0$ & $I$ \\
\hline$<\varepsilon_{f}$ & $<\varepsilon_{f}$ & Stauchungsbruch (S) & 0 & 0 & $<0$ & III \\
\hline \multicolumn{5}{|c||}{ Verankerungsbruch } & \multicolumn{5}{|c|}{ nicht erfasst } \\
\hline
\end{tabular}

Fig 7.3 Schubbruchklassifikation nach $[37,38]$ und idealplastische Theorie

Die verschwindenden Stauchungsgeschwindigkeiten $\dot{\varepsilon}_{2}$ bei Biege- und Biegeschubbruch sind natürlich so zu verstehen, dass bei längs unterarmierten Querschnitten die Neutralaxe im oder in der Nähe des Druckflansches liegt und sich somit im idealisierten Querschnitt (Fig. 6.4) am Querschnittsrand befindet (Fig. 6.8). Bezüglich des Biegeschubbruchs sei auf die Bemerkungen des Abschnittes 6.4 und die Behandlung des Lasteinleitungsproblems in Abschnitt 10.3 verwiesen. Im folgenden werden also nach der Zürcher Sprachregelung Stauchungs- (S) und StauchungsSchiebungs-(SS) Schubbrüche betrachtet, nämlich die Versuchsträger T1, T2, ET4 [40], TP2, TP4, LT 2/II [41], C1 bis C4 [38] der gut dokumentierten Stuttgarter und Zürcher Schubversuche. Sie weisen alle konstante Querkraft und Bügelarmierung auf. In Längsrichtung sind sie mit konstantem Stahlquerschnitt schlaff armiert oder mit geraden Kabeln vorgespannt. Auf die 
Verhältnisse bei verteilter Belastung, Vouten und schrägen Kabeln wird teilweise in Abschnitt 10 eingegangen.

Erste Hinweise bezüglich der Anwendbarkeit der Plastizitätstheorie auf SS/S-Schubbrüche geben das Verhälthis der Verformungen bei Bruch und Fliessbeginn und die Form des Last-Verformungs-Diagramms. Nun zeigt das Last-Durchbiegungs-Diagramm der extrem stark schubbewehrten Versuchsbalken T1, T2 einen praktisch linearen Anstieg und der Bruch erfolgte eindeutig im aufsteigenden Ast. Aber auch der sehr schwach schubarmierte Balken C3 zeigt das gleiche Verhalten (Fig. 7.4 a): Die Last-Durchbiegungs-Kurve weist nur in der Gegend der Risslast einen deutlichen Knick auf und verläuft dazwischen praktisch linear. Daraus schon den Schluss zu ziehen, die Plastizitätstheorie sei nicht anwendbar, ist aber falsch, weil die in den meisten Versuchsberichten für das Last-Verformungs-Diagramm verwendete Durchbiegung nicht die relevante Verformungsgrösse für SS/S-Brüche darstellt. Die Durchbiegungen infolge Schiebung sind auch bei sehr schwacher Bügelarmierung bis zum Bruch klein gegenüber den Durchbiegungen infolge Krümmung. Da aber bei SS/S-Brüchen die Fliessdehnung der Längsarmierung nicht erreicht wird, weist das Last-Durchbiegungs-Diagramm im wesentlichen die fast elastische Charakteristik der $M-\Phi-K u r v e$ auf.
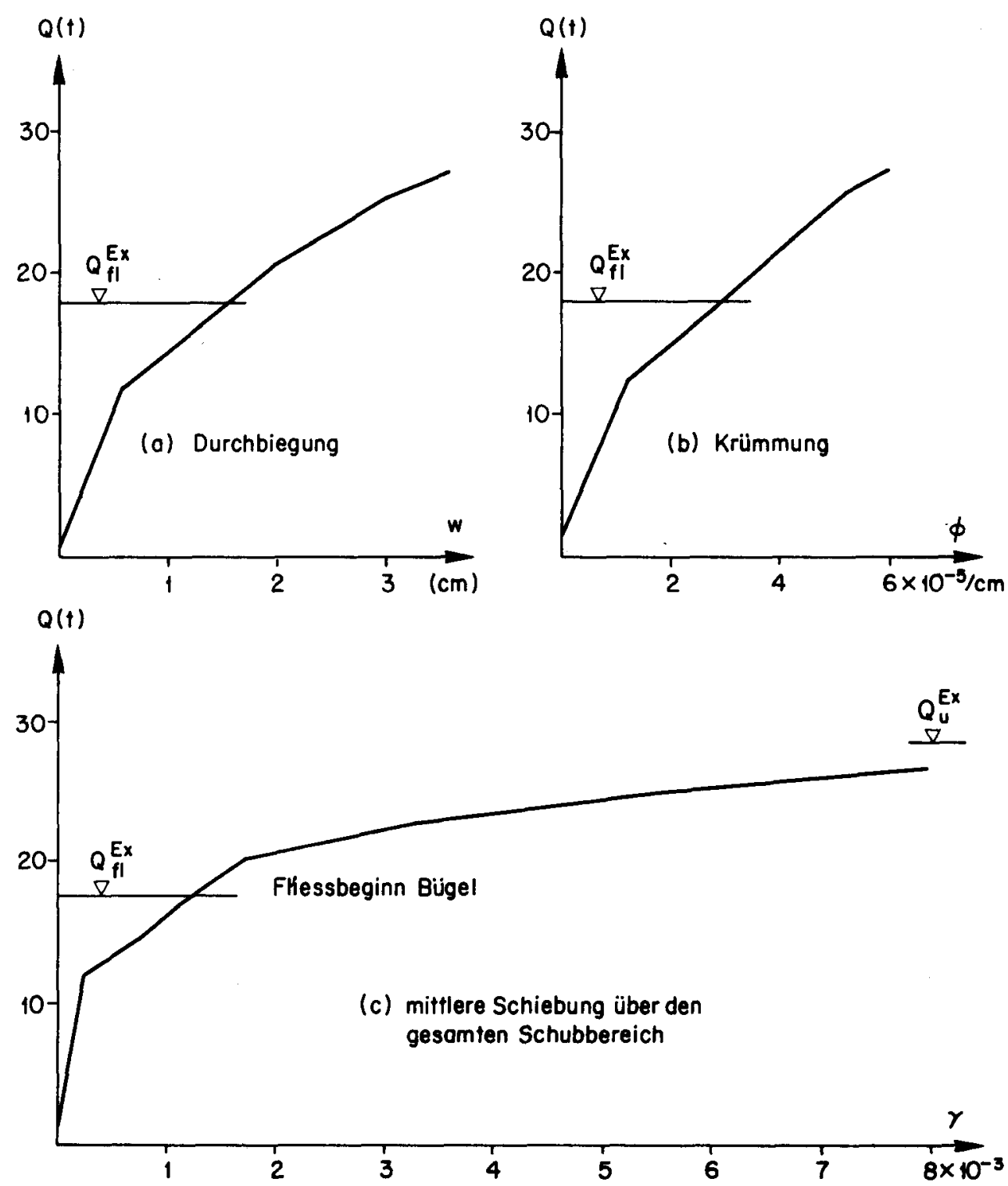

Fig. 7.4 Lost-Verformungs Diagramme Balken C 3 [38] 
In den Zürcher Schubversuchen [38] sind mittels von Flansch zu Flansch gespannter Messnetze globale Schiebungen im Schubbereich und Krümmungen im Biegebereich gemessen worden. Die gemittelten Messwerte sind in Fig. 7.4 b,c für den Balken C3 aufgetragen und bestätigen das gesagte. Wird die Querkraft gegen die für Schubversagen bei nicht massgebender Längsarmierung relevante Verformung, nämlich die globale Schiebung $\gamma$, aufgetragen, so weist das Querkraft-Verformungs-Diagramm nicht mehr eine spröde, sondern eindeutig eine elastoplastische Charakteristik auf. Insbesondere beträgt die mittlere Schiebung des Schubbereiches bei Maximallast mindestens des 6- bis 7-fache der Schiebung bei Fliessbeginn (Fig. 7.4 c). Das Ver-

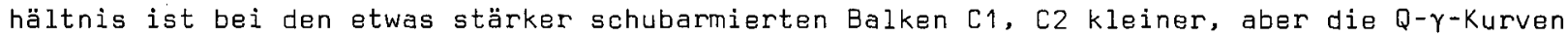
weisen immer noch elastoplastischen Charakter auf. Voraussetzung für solche Diagramme ist natürlich eine deformationsgesteuerte Belastungsvorrichtung. Man kann sich sogar fragen, ob nicht auch ein absteigender Ast zu erhalten wäre, wenn während der Deformationshalte statt der Durchbiegung Schịebungen konstant gehalten werden könnten.

In Fig. 7.5 sind einige typische Bruchbilder von SS/S-Schubbrüchen zusammengestellt. Der Vergleich von Fig. 7.5 a,b,c mit den theoretisch möglichen Mechanismen Fig. 7.2 e,f,g zeigt, dass das breite Spektrum an beobachtetem SS/S-Bruchverhalten nicht im Widerspruch steht zur Theorie. Eine stetige Biegelinie mit bis nach dem Bruch intakten Flanschen (Fig. 7.5 a) ist denkbar, denn die Verformungen des Steges klein sind, der Flansch stark, konstruktiv gut armiert und durch enge Verbügelung mit dem Steg gut verbunden ist, und wenn Bügelfliesskraft

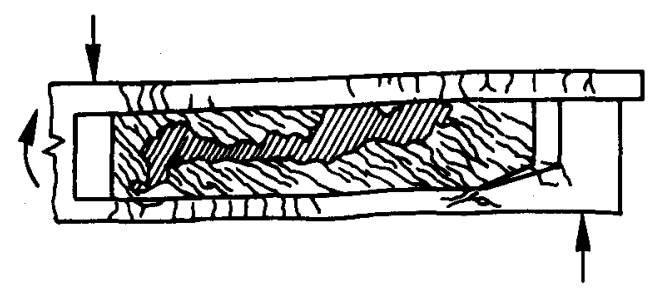

(a) TP $4[41]$

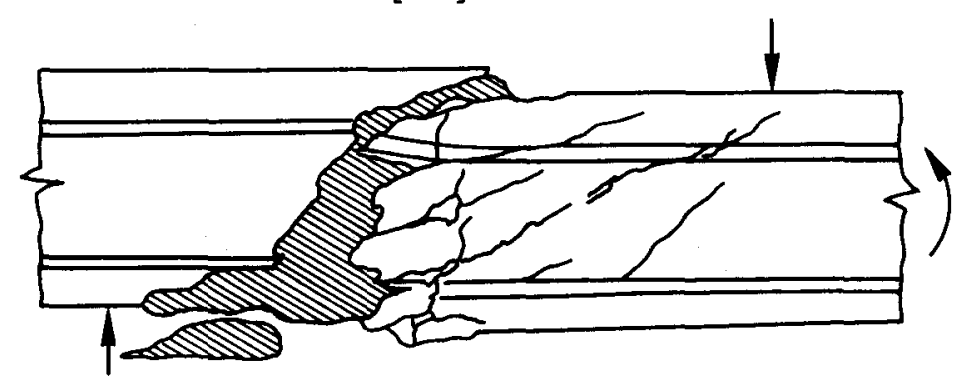

(b) C $4 R[38]$

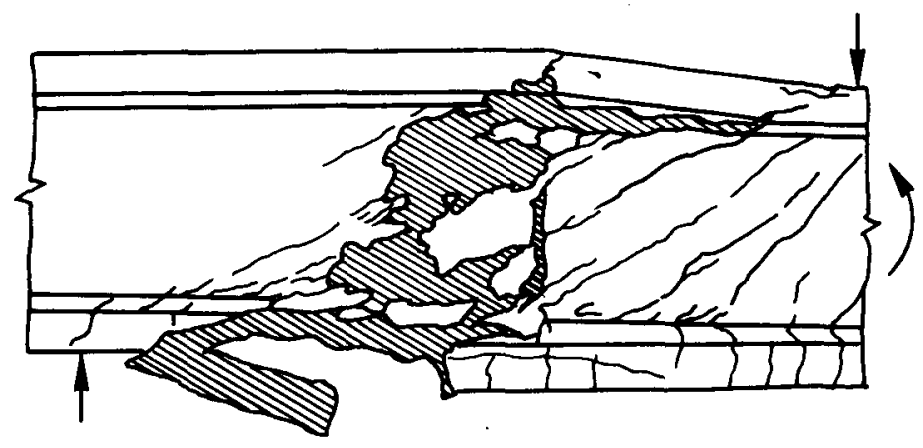

(c) C2L [38]

Fig. 7.5 Bruchbilder von Schubbrüchen infolge Versagens des Stegbetons 
und Querkraft - wie in allen hier betrachteten Versuchen - konstant sind. Ist andererseits der Flansch schwach, konstruktiv schlecht armiert, der Bügelabstand weit oder der Steg lokal durch grosse Schiebungen stark gestört, wird sich eher ein diskontinuierlicher Mechanismus einstellen (Fig. 7.5 b). Dabei kann es sich natürlich nur um rein qualitative Ueberlegungen handeln, da die theoretischen Mechanismen (Fig. 7.2) nur die inkrementale Verformung beim Erreichen der Traglast des idealplastischen Modells darstellen, während in den Bruchbildern Fig. 7.5 die totalen Verformungen des wirklichen Tragwerks sichtbar werden, und auch sekundäre Zerstörungen nicht von der primären Bruchstelle unterschieden werden können. Immerhin lässt sich den Bruchbeschreibungen [38] entnehmen, dass der Bruch, wie dies die vorliegende Theorie voraussetzt, immer vom Steg ausgeht, und die Zerstörung des Flansches als sekundär zu betrachten ist.

Nach der qualitativen Betrachtung der Verformungen sollen nun die beim Bruch erreichten Schubspannungen mit den theoretischen Werten für die "obere Schubspannungsgrenze" verglichen werden.

In Fig. 7.6 ist die auf die Betonfestigkeit bezogene maximale Schubspannung $\bar{\tau}$ in Funktion des auf die Bügelfliessspannung und Betonfestigkeit bezogenen Bügelarmierungsgehaltes $\bar{\mu}$ für Vertikalbügel und Schrägbügel aufgetragen (Gleichungen (7.16) bis (7.19)). Bei den in der Praxis meist vorkommenden Bügelarmierungsgehalten $\bar{\mu} \leqq 0.2$ sind bei gleichem Bügelvolumen Vertikalbügel theoretisch etwas günstiger als Schrägbügel. Dagegen sind bei hohen Schubarmierungsgehalten $\bar{\mu} \geq 0.2$, d.h. bei sehr schlanken Stegen, eindeutig Schrägbügel vorzuziehen. (Aufgebogene Längseisen haben im allgemeinen nicht die gleiche Wirkung, da infolge Spaltzugkräften an der Abbiegestelle ihre Wirkungsweise nicht sichergestellt ist.) Im aufsteigenden Ast der

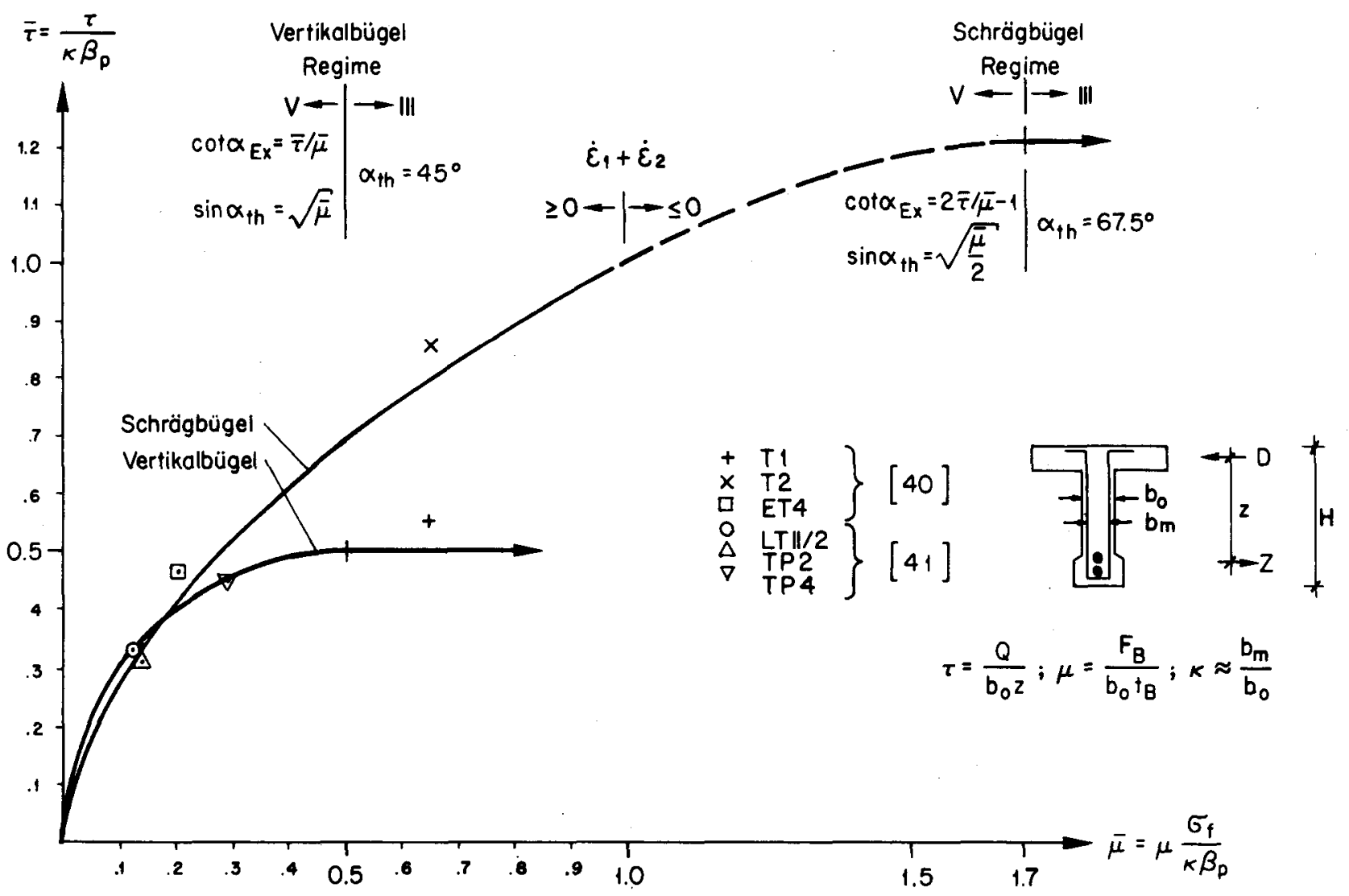

Fig. 7.6 Vergleich der theoretischen oberen Schubsponnungsgrenze mit Versuchen 
Kurven sollte die Bügelspannung die Fliessspannung erreichen, d.h. der Träger im Regime $V$ versagen. Im horizontalen Ast der Kurve dagegen sollte die Bügelspannung nicht massgebend werden und der Träger im Regime III versagen. Für die konstruktiv realisierbaren Armierungsgehalte ist letzteres offensichtlich nur bei Vertikalbügeln möglich.

Für die Interpretation der Versuchsresultate wesentlich ist schliesslich noch die Feststellung, dass Versagen im Regime $V$, d.h. im aufsteigenden Ast der Kurven, nicht bedeuten muss, dass in den Bügeln vor Erreichen der Bruchlast wesentliche plastische Deformationen gemessen werden. Alle Schub- und Torsionsversuche zeigen, dass die Kräfteumlagerungen schon bei der Rissebildung im elastischen Bereich der Armierung einsetzen und nicht erst mit dem Fliessen einer Armierung. Der Aufbau der Bügelspannungen hängt somit von der Anrissrichtung und von den Steifigkeitsverhältnissen zwischen Riss- und Fliesslast ab und ist insbesondere vom vorspanngrad abhängig. Ist die Anrissrichtung und/oder die sich zwischen Riss- und Bügelfliesslast einstellende Richtung der Betondrucktrajektorien (z.B. berechnet nach [16]) ungefähr gleich gross wie die für den Kollapszustand berechnete, so werden Bügel und Betondruckspannungen ihre Grenztragfähigkeit ungefähr gleichzeitig erreichen. Ein solches Schubversagen würde nach der engen Definition in [37] als S-Bruch bezeichnet. Für SS-Brüche werden plastische Deformationen in den Bügeln vorausgesetzt, weil in diesem Fall eine durch die grossen Schiebungen bedingte Abminderung der Betonfestigkeit angenommen wird.

Damit ist das Hauptproblem bei der Auswertung der Versuchsergebnisse angesprochen, nämlich die Frage, welche Querschnittswerte und Materialkennwerte der Berechnung von $\bar{\tau}, \bar{\mu}$ zugrunde zu legen sind. Als Fliessspannung $\sigma_{f}$ der Armierung wird wie üblich die Streckgrenze $\sigma_{0.2 \%}$ verwendet. Für die Höhe h des idealisierten Querschnittes ist aus der Sicht sowohl der Schubspannungsverteilung über den Querschnitt als auch der Beanspruchung der Längsarmierung der Hebelarm der inneren Kräfte $z$ einzusetzen entgegen eventuell anderer Regelungen in Schubnormen.

Bei der Betonkonstanten $P_{b}=k b_{0} \beta$ stellt sich die Frage nach der mitwirkenden Stegdicke $b_{m}<b_{0}$ und nach der Betondruckfestigkeit $\beta$. Sicher ist von der Prismenfestigkeit $\beta=\beta_{p}$ auszugehen. Die entsprechenden Messwerte streuen jedoch stark, werden meist nur an wenigen Prabekärpern ermittelt und sind von der Versuchsdauer bzw. Belastungsgeschwindigkeit abhängig. Zudem entsprechen die Versuchsbedingungen eines Prismenversuchs auch kaum den Verhältnissen in einem gerissenen, armierten Steg.

In den Stuttgarter Schubversuchen sind die maximalen vor SS/S-Versagen gemessenen Betondruckspannungen kleiner als die angegebene mittlere Prismendruckfestigkeit. In [41] wird dies auf die Reduktion der zweiaxialen Betonfestigkeit (Fig. 4.3 a) durch die Querzugsspannungen in den Betondruckstreben zwischen den Rissen zurückgeführt. Gestützt auf Versuche, in welchen von unter Zug stehender Armierung gekreuzte Betonprobekörper geprüft wurden [43], werden Abminderungsfaktoren $0.7-0.8$ ermittelt. Die reduzierten Festigkeiten werden dann (TP4) mit den am $30^{\circ}$-Fachwerk (d.h. unter Vernachlässigung von Zugspannungen) berechneten Betondruckspannungen verglichen. Dieses Vorgehen ist natürlich mit der hier vorausgesetzten Plastizitätstheorie nicht vereinbar, da so tiefere Traglasten resultieren, obschon die quadratische Betonfliessfigur in derjenigen nach Fig. 4.3 a enthalten ist. Der Uebergang von Fliessfigur 2.1 zu der ausgezogenen von Fig. 4.3 a beeinflusst zudem die theoretischen werte für die Traglast und Betondruckspannung beim kollaps nur im Bereich kleiner Drucktrajektorienneigungen $\alpha$ bzw. kleiner $\bar{\mu}$ : Aus der kinematischen Restriktion $\dot{\varepsilon}_{x}=0$ wegen des Nichtfliessens der Längsarmierung folgt für $30^{\circ} \leq \alpha \leq 45^{\circ}$ sofort $1 \leqq \dot{\varepsilon}_{1} /\left(-\dot{\varepsilon}_{2}\right) \leq 3$. Mit diesen Dehnungsgeschwindigkeiten ist aber' auch aufgrund der Fliessfigur 4.3 a nur der spannungszustand $\sigma_{1}=0, \sigma_{2}=-\beta_{p}$ verträglich, solange die grundlegende Beziehung (3.1) der Plastizitätstheorie vorausgesetzt 
wird. Wegen des Fehlens der kinematischen Restriktion $\dot{\varepsilon}_{x}=0$ sind die erwähnten Versuchsresultate [43] aus dieser Sicht auch nicht ohne weiteres auf die Verhältnisse im Steg bei SS/S-Schubbrüchen übertragbar.

Ueber die Genauigkeit bzw. Aussagekraft des Absolutwertes der Prismenfestigkeit darf man sich keine Illusionen machen. Zur Illustration sei auf die Grössenordnung der Unterschiede zwischen statischem und dynamischem Wert der Prismenfestigkeit am Beispiel der Mittelwerte für die Torsionsversuche [36] hingewiesen.

\section{Kraftgesteuert:}

- EMPA, Kurzzeitversuch

$$
B_{p}=383 \mathrm{~kg} / \mathrm{cm}^{2}=115 \%
$$

Dehnungsgesteuert, Dehnungsschritt $0.210^{-3} / 2 \mathrm{~min}$

- dynamisch

- statisch Dehnungsgehalt pro Schritt

$$
\begin{array}{ll}
\text { pro schritt } & \beta_{p d}=334 \mathrm{~kg} / \mathrm{cm}^{2}=100 \% \\
2 \mathrm{~min} & \beta_{p s}=299 \mathrm{~kg} / \mathrm{cm}^{2}=90 \%
\end{array}
$$$$
30 \mathrm{~min} \quad \beta_{p s}=267 \mathrm{~kg} / \mathrm{cm}^{2}=80 \%
$$

Diese Werte (ohne $\left.\beta_{p s}(30 \mathrm{~min})\right)$ sind auch für die Zürcher Schubversuche bekannt. Da aber hier keine Dehnungen an den Betondruckstreben gemessen wurden, sind keine direkten Rückschlüsse möglich.

Beim Kollaps bildet sich ein Mechanismus über die gesamte Steghöhe aus (Fig. 7.5). Es ist aber kaum anzunehmen, dass die beim Kollaps erreichten totalen Dehnungen überall gleich sind. Dies bedeutet, da das Spannungs-Dehnungs-Diagramm des Betons in Wirklichkeit nicht idealplastisch ist, dass sich der Betonspannungszustand in einzelnen Bereichen des Mechanismus auf dem aufsteigenden, in anderen auf dem absteigenden Ast des $\sigma-\varepsilon$-Diagramms befindet. Dies äussert sich aber ebenfalls in einem reduzierten, mittleren Bruchwiderstand.

Schliesslich sei auch noch eine Ueberlegung betreffend der mitwirkenden Stegdicke $b_{m} \leq b_{0}$ zur Diskussion gestellt. Das Versagen des Stegbetons geht erwiesenermassen von durch Risse und grosse Schiebungen stark gestörten Zonen aus [38] und kündigte sich z.B. beim Versuch [2L schon frühzeitig durch lokales Abblättern der Betonoberfläche an. Ob die sich beim Bruch lösende Betonüberdeckung der Bügel für den Bruchwiderstand und das globale Kräftespiel im Bruchzustand (Drucktrajektorienneigung $a$ ) noch voll mitwirkend in Rechnung gestellt werden darf, ist deshalb fraglich. Die Dehnungsmessungen auf dem Beton dagegen stammen tendenziell aus schwach gestörten Zonen, wo die Messbolzen nicht ausgefallen sind, und sind somit eher mit den Rechenwerten bei "vollem Querschnitt der Betondruckstreben" zu vergleichen. Rechnerisch äussert sich natürlich eine Reduktion von bo in (7.16), (7.17) genau gleich wie eine Reduktion von $\beta_{p}$.

Die Auswertung erfolgte angesichts der angedeuteten Unsicherheiten möglichst einfach: Die in Fig. 7.6 eingetragenen Werte $\bar{\tau}, \bar{\mu}$ für die Stuttgarter Versuche sind mit den nichtreduzierten Prismenfestigkeiten gemäss [40, 41] berechnet, aber ohne Mitwirkung der Betonüberdeckung der Bügel entsprechend einem $k=\left(b_{0}\right.$ - Ueberdeckung $) / b_{0}=0.7 \div 0.85$ (Rechenwerte siehe Anhang B). Sie folgen mit der zu erwartenden Bandbreite den theoretischen Kurven und bestätigen diese in ihrem generellen Verlauf.

Neben den quantitativen ist aber vor allem auch den qualitativen Aspekten Beachtung zu schenken. Hier sind besonders die zwei bis auf die Richtung der Bügel identischen, volumengleich 
armierten Balken T1, T2 zu erwähnen. Während im Versuchsträger T2 die Schrägbügel mit rund $4400 \mathrm{~kg} / \mathrm{cm}^{2}$ die Fliessgrenze von $4300 \mathrm{~kg} / \mathrm{cm}^{2}$ theoriegemäss (Regime $V$ ) erreichten, blieben die Vertikalbügel von $T 1 \mathrm{mit} 3300 \mathrm{~kg} / \mathrm{cm}^{2}$ praktisch im theoretischen Verhältnis $0.5 / \bar{\mu}$ unter der Fliessspannung, und der Träger versagte unter $2 / 3$ der Bruchlast von 12 in Vebereinstimmung mit (7.4b) im Regime III. Armierungsgehalte $\bar{\mu} \geqq 1 / 2$ sind offensichtlich in der form von Vertikalbügeln nicht sinnvoll. Schliesslich bestätigen sich die theoretischen Neigungen der Betondrucktrajektorien beim Kollaps $\alpha_{1}=45^{\circ}(T 1), \alpha_{2}=31 \div 34^{\circ}(T 2)$ auch im Verhältnis der gemessenen Betondruckspannungen im Steg. In [40] wird speziell darauf hingewiesen, dass die Druckspannungen im schrägbügelbewehrten T2 nur um einen Faktor 1.5 kleiner waren als diejenigen im Träger T1 mit Vertikalbügeln, während nach dem $45^{\circ}$-Fachwerkmodell ein Faktor 2 zu erwarten wäre. Berechnet man jedoch das theoretische Spannungsverhältnis unter Benutzung der Drucktrajektorienneigungen im Kollapszustand - was angesichts der mit der Last bis zum Bruch praktisch linear anwachsenden Spannungen vertretbar erscheint - , so ergibt sich auch theoretisch ein Faktor von rund 1.5:

$\frac{n_{2}(T 1)}{n_{2}(T 2)}=\frac{n_{x y}\left(\sin \alpha_{2} \cos \alpha_{2}+\sin ^{2} \alpha_{2}\right)}{\sin \alpha_{1} \cos \alpha_{1} n_{x y}}=1.43 \div 1.57$

Berücksichtigt man noch, dass auch die Voraussetzung (7.6) bzw. (7.14) über die gesamte Trägerlänge erfüllt ist, so bestätigen die zwei Träger T1, T2 sehr schön, dass es keine obere Schubspannungsgrenze, sondern nur einen oberen Grenzwert für die Betondruckspannungen im Steg gibt.

Ebenfalls erreicht worden ist die Bügelfliessspannung in den ausschliesslich mit Vertikalbügeln armierten Trägern TP2, ET4 und bei LT II/2 zumindest in der Umgebung der allerdings bewusst zu schwach bemessenen Zone der indirekten Krafteinleitung über einen Querträger. Bei TP4 betrug die grösste gemessene Bügelspannung $3870 \mathrm{~kg} / \mathrm{cm}^{2}$ unter $95 \%$ der Bruchlast. Extrapoliert man zur Bruchlast, ergibt sich ein Wert, der nahe an der Streckgrenze von $4310 \mathrm{~kg} / \mathrm{cm}^{2}$ liegt. Im Versuchsbericht [41] wird mit aus dem Rissebild ermittelten Druckdiagonalenneigungen $30 \div 36^{\circ}$ gerechnet. Diese flache (Träger vorgespannt), sich schon unterhalb der Bügelfliesslast einstellende Drucktrajektorienneigung entspricht aber ziemlich genau der theoretischen Neigung $\alpha=33^{\circ}$ beim Kollaps. Es ist also auch theoretisch zu erwarten, dass Bügel und Stegbeton ihre Grenztragfähigkeit ungefähr gleichzeitig erreichen.

Da die minimale theoretische Mechanismuslänge $h \cot 2 \alpha$ bei allen Versuchsträgern kürzer ist als der Abstand Auflager - Einzellast, handelt es sich bei den Kurven in Fig. 7.6 sicher um obere Grenzwerte im Sinne der Scheibentheorie. Als untere Grenzwerte im Sinne der Scheibentheorie dürfen sie nicht betrachtet werden, solange nicht statisch zulässige Scheibenspannungszustände im Bereich der konzentrierten Lasten gefunden sind. Dagegen handelt es sich um untere Grenzwerte im Sinne der Balkentheorie, wenn die Längsarmierung in allen Querschnitten die zusätzlichen Zugkräfte infolge Querkraft (7.6) bzw. (7.14) aufnehmen können, und somit in keinem Querschnitt die Balkenfliessbedingungen (6.15) des Regimes I verletzt werden. Dies ist für die Träger T1, T2, ET4 über die gesamte Trägerlänge, für TP2, TP4 bis etwa zu einem Abstand h und für LT II/2 bis zu einem Abstand 1.7h von der Einzellast der Fall.

In Fig. 7.7 sind die Resultate der Zürcher Schubversuchsserie C [38] ausgewertet für verschiedene Kombinationen der Materialkennwerte: statische oder dynamische Prismenfestigkeit, volle oder teilweise Mitwirkung der Stegdicke im Kollapszustand, Streckgrenze oder maximale gemessene Bügelspannung (Rechenwerte siehe Anhang B). Auch durch diese Versuchsresultate wird der generelle Verlauf der theoretischen Kurve bestätigt, unabhängig von der Frage, mit welchen spezifischen Materialkennwerten sich quantitativ die beste Uebereinstimmung ergibt. 


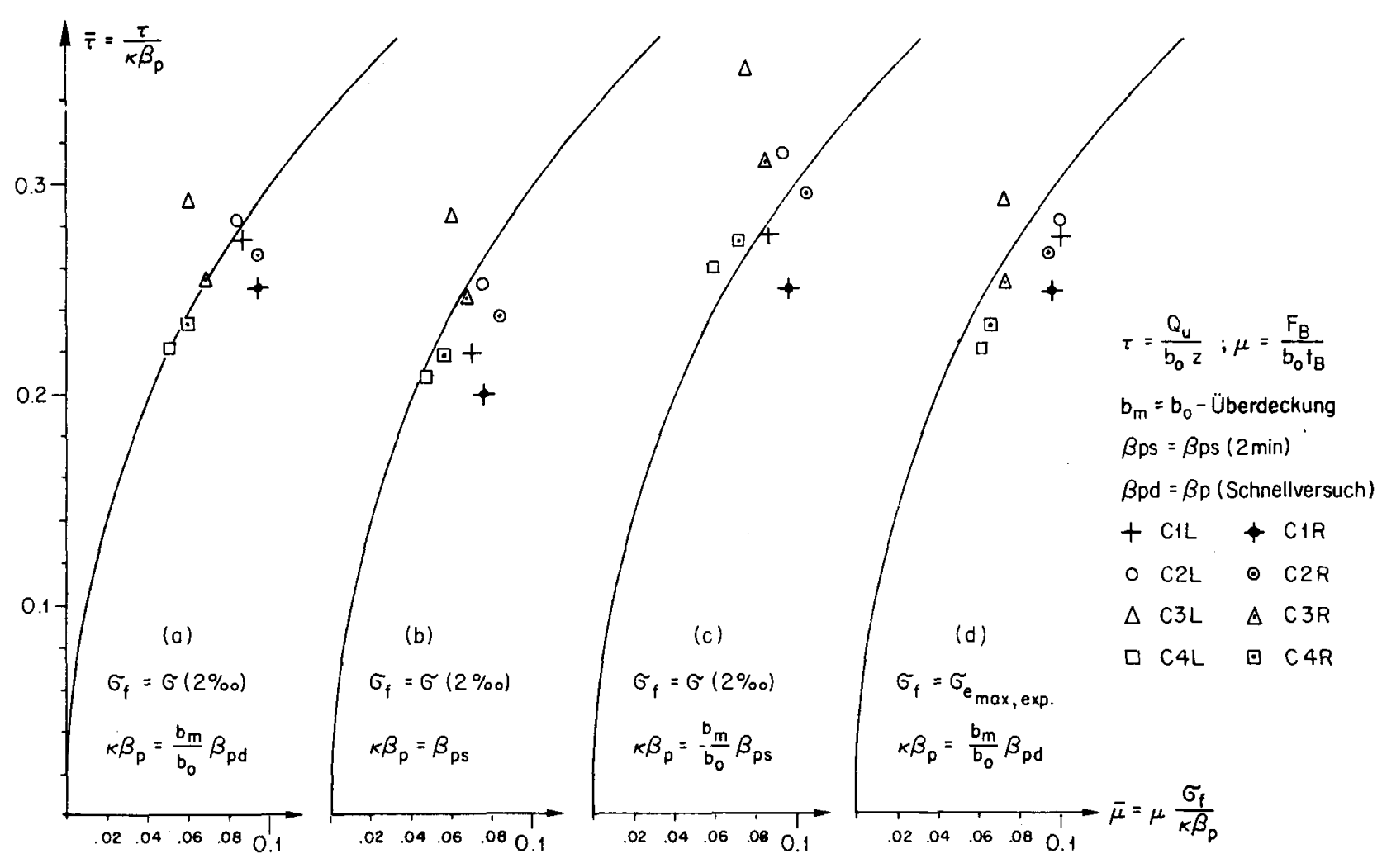

Fig. 7.7 Vergleich der theoretischen oberen Schubspannungsgrenze mit Versuchen [38]

Sieht man von den Schubbrüchen C3L und C1R ab, so ist kein signifikanter Unterschied im Schubtragverhalten der Träger [1, C2 gegenüber den Trägern C3, C4 mit verdoppelter Flanschdicke festzustellen. Dies stützt die These, dess nur der bis zu den Zug-/Druckresultierenden in die Flanschen fortgesetzt gedachte Steg für die Schubtragfähigkeit bei nicht massgebender Längsarmierung bestimmend ist. Bei den stark nach oben bzw. unten streuenden Schubbrüchen C3L und C1R sind dicker Druckflansch und enger Bügelabstand bzw. dünner Druckflansch und weitester Bügelabstand miteinander kombiniert, was auf die konstruktive Bedeutung der Flanschen für die Verhinderung eines vorzeitigen Ausbrechens der Betondruckdiagonalen zwischen den Bügeln hinweist.

Entsprechend den kleinen $\bar{\mu} \ll 0.5$ haben die Vertikalbügel in allen Trägern die Fliessdehnung stark überschritten. Die minimale Mechanismuslänge $h \cot 2 \alpha$ ist überall kleiner als der Abstand Auflager - Einzellast, und die theoretischen Kurven sind zumindest obere Grenzwerte. Dagegen kann die vorhandene Längsarmierung die bei diesen kleinen $\bar{\mu}$ bzw. flachen Drucktrajektorienneigungen a sehr grossen, theoretischen Zusatzzugkräfte infolge Querkraft schon z.T. ab der halben Schubspannweite nicht mehr aufnehmen. Da jedoch in Wirklichkeit die Längsarmierung die Fliessgrenze nicht erreicht hat, muss auf eine erhebliche sprengwerkwirkung geschlossen werden, die die Längsarmierung im Bereich der Einzellast auf Kosten derjenigen im Auflagerbereich entlastet. In die gleiche Richtung weist die Suche nach zulässigen Scheibenspannungszuständen im Bereich der Lasteinleitung.

Sprengwerkwirkung kann natürlich innerhalb der Balkentheorie nach Fig. 6.3 nicht erfasst werden, da die Ableitung der Querschnittswiderstände immer auf der Voraussetzung $N_{y}=0$ beruiht. Sprengwerkwirkung ist ein typisches Scheibenproblem. Die Serie C ist deshalb auch mit dem Scheiben-Programm WALL [48] berechnet worden, und die Resultate zeigen, dass die Längsarmierung bei Berücksichtigung der Scheibenwirkung auch theoretisch zur Erreichung der beobachteten Schubbruchlasten genügt. Entsprechende Resultate haben sich bei der Nachrechnung von Schub- 
versuchen an Stahlbetonbalken mit geneigten Spannkabeln mittels WALL ergeben [39]. Sprengwirkung ist in Abschn. 11 auch analytisch am Beispiel des "shear wall coupling beam 391" behandelt.

Wie Fig. 7.7 zeigt, liefert aber der in diesem Fall als Mechanismusmethode zu betrachtende Ansatz auch bei vermuteter Sprengwerkwirkung eine akzeptable Näherung für die obere, nominelle Schubspannungsgrenze der Serie $C$. Zudem ist der tatsächliche Wert der oberen Schubspannungsgrenze bei diesen kleinen $\bar{\mu}$ für die praktische Dimensionierung nicht besonders interessant, da hier nicht der Grenzzustand "Tragfähigkeit", sondern der Grenzzustand "unzulässige Rissbreiten unter Gebrauchslast" massgebend wird. Gemäss Erläuterung zur neuen Richtlinie 34. der Norm SIA 162 [25] wird zufriedenstellendes Risseverhalten im Gebrauchszustand erst erreicht, wenn die Dimensionierung der Bügelarmierung mit "Diagonalenneigungen". $\tan \alpha \geq 0.6, \alpha \geqq 30^{\circ}$ erfolgt, obwohl von der Tragfähigkeit her tan $\geqq 0.5$ genügen würde. Dámit wird die Dimensionierung auf $\bar{\tau} \geqq(5 / 3) \bar{\mu}$ ausgeschlossen.

Zusammenfassend kann festgestellt werden, dass der plastizitätstheoretische Ansatz für schlaff armierte (T1, T2, ET4) und vorgespannte Träger mit unterschiedlicher relativer Druckflanschstärke über den weiten Bereich des Schubarmierungsgehaltes $\bar{\mu}=0.06 \div 0.6$ und der Betonfestigkeiten $\beta_{p}=220 \div 450 \mathrm{~kg} / \mathrm{cm}^{2}$ die obere Schubspannungsgrenze generell zutreffend beschreibt und somit die wesentlichsten Parameter erfassen dürfte. Es dürfte sich deshalb lohnen, einerseits von der Theorie her auch statisch zulässige Scheibenspannungszustände zu suchen, und andererseits Versuche durchzuführen, die speziell auf die Verifikation/Felsifikation der vorliegenden Theorie und auf die Ermittlung der massgebenden Materialkennwerte für das Stegbetonversagen hin konzipiert sind.

In Fig. 7.8 wird der plastizitätstheoretische Ansatz mit der Richtlinie 34 Norm SIA 162 [29] für einen Beton BH 300 verglichen. Dabei sind die verschiedenen Korrekturspickel im Bereich kleiner $\bar{\tau}$ im Uebergang zum ungerissenen Bereich übersichtlichkeitshalber weggelassen. Die Geraden $\tan \alpha=3 / 5, \tan \alpha=5 / 3, \tau=6 \tau_{\mathrm{r}}$ begrenzen den Bereich der "Spannungszustände" $\bar{\tau}, \bar{\mu}$, auf welche armiert werden darf. Setzt man für den Abminderungsfaktor $k$ näherungsweise das oben erwähnte Verhältnis der statischen Prismenfestigkeit bei 30-minütigem Dehnungsgehalt zur Prismennennfestigkeit aus dem üblichen Kurzzeitversuch ein, $\kappa \simeq 80 \% / 115 \%=0.7$, und beachtet man zudem, dass in der Norm zur Berechnung von $\tau$ die volle Stegbreite und der Abstand der von den Bügeln umschlossenen Längseisen eingesetzt werden darf, so zeigt Fig. 7.8 a klar, dass die neue Richtlinie für Vertikalbügel ziemlich nahe an die praxisgerechte Linearisierung des theoretischen Viertelkreises durch ein Viertelsechseck kommt.

Aus der Sicht des plastizitätstheoretischen Ansatzes für die obere Schubspannungsgrenze darf also die Richtlinie 34 für Vertikalbügel als moderne Norm bezeichnet werden, die das tatsächliche Materialverhalten relativ gut erfasst und nicht mehr allzuviel überflüssige Sicherheitsreserven enthält. Damit ist aber auch die Notwendigkeit aufgezeigt, die massgebenden Grössen für den plastischen Widerstand des Stegbetons $P_{b}=k b_{0} \beta_{p}$ exakter zu untersuchen. Mit der Elimination von überflüssigen Sicherheitsreserven entsteht auch die Notwendigkeit, früher vernachlässigte Einflüsse zu berücksichtigen. In Bereichen, wo der Steg durch zusätzliche Querbiegemomente beansprucht ist, oder wo konzentrierte Lasten eingeleitet werden (Abschnitt 10.3), kann die obere Schubspannungsgrenze (7.18) je nach der eingelegten Bügelarmierung unter Umständen nicht voll ausgenützt werden. Ebenfalls nicht untersucht worden ist das Schubproblem in Stützen unter hohen Axiallasten.

Wesentlich andere Verhältnisse liegen bei den allerdings selten verwendeten Schrägbügeln vor, wie Fig. 7.8 b zeigt. Hier ist die Richtlinie 34 sehr konservativ. Für überwiegend ruhende Belastung können mit Schrägbügeln wesentlich schlankere Stege gebaut werden als dies die Norm 


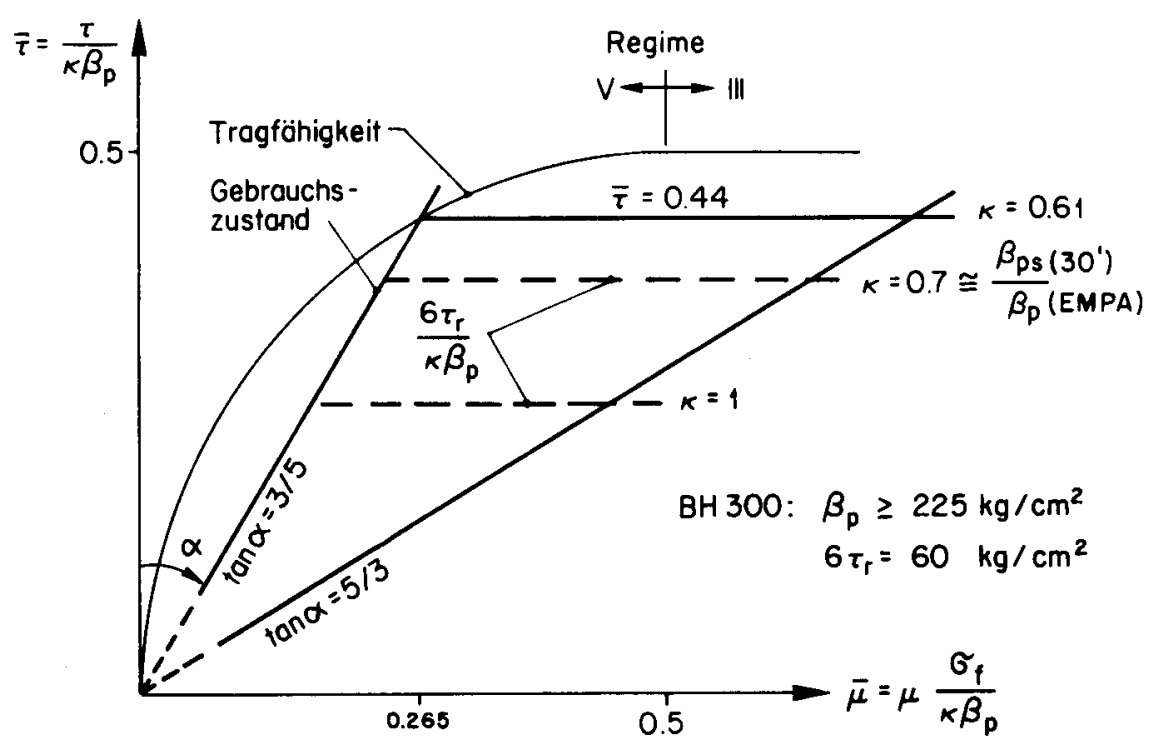

a) Vertikalbügel

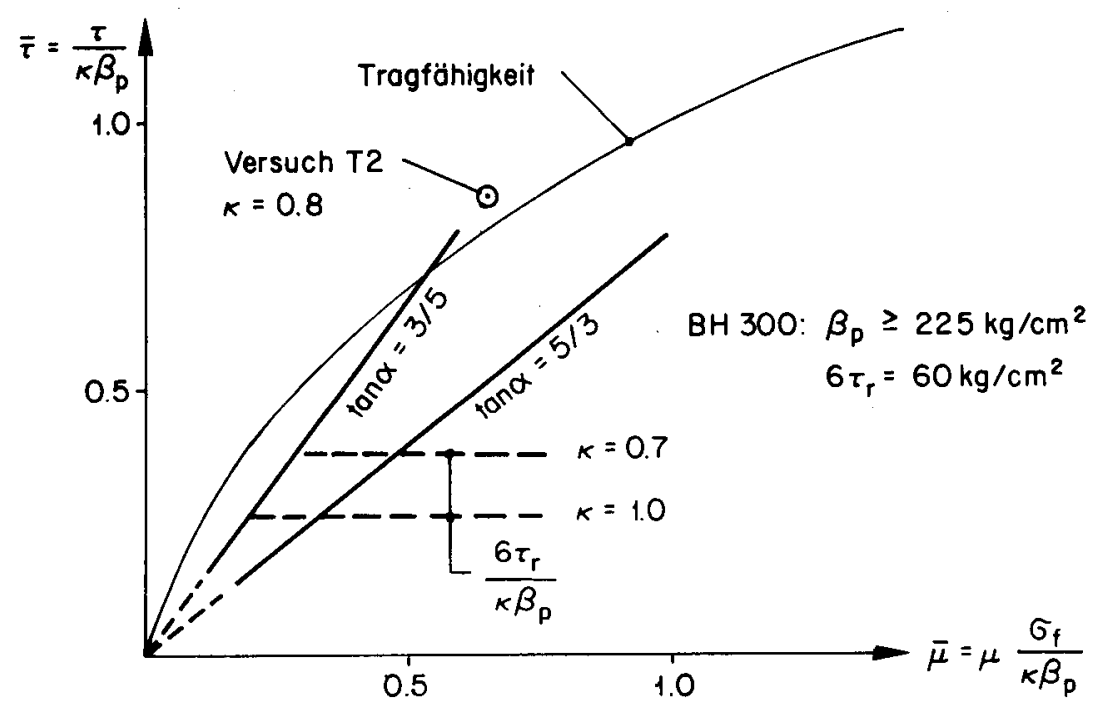

b) Schrägbügel $\left(45^{\circ}\right)$

Fig. 7.8 Vergleich Theorie SIA-Norm 162 RL 34 [29] 
zulässt: Will man dem Konstrukteur diese Freiheit lassen und für Schräg- und Vertikalbügel gleiche Sicherheiten gegen Stegbetonversagen einführen, so ist neben der Beschränkung der Diagonalenneigung nicht die Schubspannung, sondern die Betondruckspannung im Steg zu begrenzen. Damit werden auch gleichmässigere Sicherheiten für unterschiedliche Bügelarmierungsgehalte erzielt. Ueber die Betondruckspannung erhält der Konstrukteur zudem ein wesentlich besseres Gefühl für das Kräftespiel und die tatsächliche Beanspruchung im Steg als über die in gerissenem armiertem Beton nicht direkt relevante Grösse Schubspannung.

Damit stellt sich auch die Frage nach der Abstufung der obern Schubspannungsgrenze mit der Betonfestigkeit. Diese ist in der Richtlinie 34 als Vielfaches von $\tau_{r}$ ausgedrückt. Mit $\tau_{r}$ wird ungefähr der Uebergang vom ungerissenen zum gerissenen Zustand erfasst. Dieser Wert ist somit proportional der Zugfestigkeit des Betons abgestuft, also proportional $\sqrt{\beta_{p}}$. Dies führt nun aber für verschiedene Betonqualitäten $z u$ unterschiedlichen Abständen von $6 \tau_{\Gamma} / K \beta_{p}$ von der horizontalen Sechseckseite $\bar{\tau}=0.44$ in Fig. 7.8 a. So entspricht $\bar{\tau}=0.44$ für einen $B H 300$ einem Abminderungsfaktor $K=0.61$ (Fig. 7.8 a), für einen BH 200 jedoch nur einem solchen von $k=0.73\left(6 \tau_{r}=48 \mathrm{~kg} / \mathrm{cm}^{2}, \beta_{P}=150 \mathrm{~kg} / \mathrm{cm}^{2}\right)$.

Akzeptiert man jedoch, dass die obere Schubspannungsgrenze in armiertem Beton primär von der Druckfestigkeit und nicht von der Zugfestigkeit des Betons abhängt, so dürfte die obere Schubspannungsgrenze bzw. ein eventueller Grenzwert für die Betondruckspannungen im Steg proportional $\beta_{p}$ abgestuft werden, wie dies für die zulässigen Schwerpunkts- und Randdruckspannungen der fall ist. Dabei darf man sich auch nicht von der formel (7.4a) täuschen lassen. Dimensioniert man auf eine bestimmte Druckdiagonalenneigung $\alpha$ im Kollapszustand, so stehen die erforderlichen $P_{y}$ und $P_{b}$ gemäss (7.5a) in festem Verhältnis, und $Q$ variiert in (7.4a) proportional $B_{p}$. Hier nicht weiter untersucht wird die Frage, ob eine $\beta_{p}$ proportionale Abstufung vom konstruktiven Standpunkt und in bezug auf die Verbundspannungen der Längsarmierung verantwortbar ist. Die Versuchsträger IP4 $\left(\beta_{p}=450 \mathrm{~kg} / \mathrm{cm}^{2}\right.$ ) und $I P 3$ (Biegebruch; bis auf die erhöhte Betonfestigkeit $\beta_{p}=494 \mathrm{~kg} / \mathrm{cm}^{2}$ identisch mit IP2] [41] zeigen diesbezüglich kein schlechteres Verhalten, sind aber natürlich als Versuchsträger konstruktiv besonders sorgfältig durchgebildet. 
Zur Erfassung des Bruchwiderstandes unterarmierter Stahlbetonbalken mit geschlossenem Querschnitt unter Torsion und Biegung sind zwei Ansätze bekannt, die auf der Plastizitätstheorie beruhen: Das räumliche Fachwerkmodell [18, 20, 22] und der "Skew Bending"-Mechanismus [17]. In Abschnitt 8.1 werden diese beiden Ansätze kurz rekapituliert. Es zeigt sich, dass plastizitätstheoretisch strenge Kollapsmechanismen für Stahlbetonbalken unter Torsion und Biegung noch fehlen. Insbesondere ist auch kein mit dem im Fachwerkmodell vorausgesetzten Spannungszustand verträglicher Mechanismus bekannt.

Die Entwicklung solcher Mechanismen, Thema der Abschnitte 8.2 bis 8.5 , ist in zweifacher Hinsicht von Bedeutung. Einerseits zeigen die Mechanismen auf, mit welchen kinematischen Annahmen der Balkentheorie der Spannungszustand des Fachwerkmodells verträglich ist. Damit wird der Gültigkeitsbereich der entsprechenden Querschnittsinteraktionsformeln klarer erkennbar. Zudem ist, da in Versuchen primär Verformungen gemessen werden, die Berechtigung von Verformungsannahmen grundsätzlich auch einfacher nachweisbar als diejenige von statischen Annahmen. Andererseits genügen zur Ueberprüfung der Frage, ob die Plastizitätstheorie überhaupt auf Stahlbetonbalken anwendbar ist, bloss untere Grenzwerte für die Traglast nicht. Dazu sollten die Versuchsergebnisse mit der plastizitätstheoretisch exakten Traglast oder zumindest mit einem durch untere und obere Grenzwerte genügend eingeschränkten Bereich für die exakte Traglast verglichen werden können.

Die Abschnitte 8.2 bis 8.4 sind auf Balken mit dünnwandigem, geschlossenem Querschnitt unter konstantem Biege- und Torsionsmoment beschränkt. Auf Vollquerschnitte, Wälbtorsion und das Problem des Einbezuges der Querkraft wird in Abschnitt 8.5 eingegangen. Auszüge aus dem Abschnitt 8 sind in [24] veröffentlicht worden.

\subsection{Bisherige plastizitätstheoretische Ansätze}

Das Fachwerkmadell und der Zusammenhang zwischen Fachwerkmodell und einfacher Balkentheorie (Fig. 6.3) sind in Abschnitt 6 für Biegung und Querkraft ausführlich behandelt worden. Die Resultate gelten sinngemäss auch für das räumliche Fachwerkmodell für Balken mit dünnwandigem, geschlossenem Querschnitt unter Torsion und Biegung. Dieses wird im folgenden anhand eines einfachen Beispiels rekapituliert, auf das später jeweils wieder zurückgegriffen werden kann. Anschliessend werden der "Skew Bending"-Mechanismus diskutiert und die Gründe für die Widersprüche zwischen diesem und dem Fachwerkmodell aufgezeigt.

In den Arbeiten [18, 20] werden die Bügel und die in den vier Querschnittsecken konzentriert gedachte Längsarmierung des Balkens von Fig. 8.1 a als Zugpfosten und Eckstringer eines räumlichen Fachwerks aufgefasst, in welchem der Beton die Funktion der Druckdiagonalen übernimmt. Es wird angenommen, dass sich der Beton im Kollapszustand in einem einaxialen und in den einzelnen Schubwänden konstanten Druckspannungszustand befindet. Drucktrajektorienneigung $\alpha$ und Schubfluss $S$ sind somit entlang jeder Querschnittsseite konstant, und die Resultierende $D$ der Betonspannungen einer Querschnittsseite greift in deren Schwerpunkt unter der Neigung $a$ an. Weiter wird vorausgesetzt, dass für konstantes Biege- und Torsionsmoment die Eckstringerkräfte konstant sind oder gleichwertig, dass der Schubfluss 5 konstant über den Querschnittsumfang ist. Schliesslich beschränkt sich die Theorie auf unterarmierte Balken, bei welchen kein Versagen des Betons eintritt, bevor der Kollaps durch Fliessen der Längs- und Bügelarmierung eingeleitet wird. Im Falle konvexer, polygonaler Querschnitte bedeutet dies, 
dass im Kollapszustand höchstens die Armierung einer Querschnittsseite nicht an der Fliessgrenze liegt.

Im vorliegenden Beispiel sollen die vier Eckstringer die gleiche Fliesskraft $Z_{f}$ aufweisen. Die Bügelfliesskraft pro Längeneinheit betrage $P_{u}$ in der unteren und oberen Schubwand und $P_{s}$ in den seitlichen Schubwänden. Für positives Biegemoment fliesst dann höchstens die Armierung der oberen Schubwand nicht und Biege- und Torsionsmoment im Kollapszustand werden mit Vorteil aus den Momentengleichgewichtsbedingungen bezüglich der oberen Querschnittsseite ermittelt.

$M=2 z_{f} h-2 \operatorname{shcot} \alpha_{s} \frac{h}{2}-s b \cot \alpha_{u} h$,

$T=2 S h \frac{b}{2}+S b h=2 b h S=2 F_{D} S$.

Das Vertikalgleichgewicht von aus den drei plastifizierten Schubwänden ausgeschnittenen "free bodies" verlangt

$S=P_{S} \cot \alpha_{S}=P_{U} \cot \alpha_{U}$,

wie Fig. 8.1 b für den Fall einer seitlichen Schubwand zeigt. Insbesondere weisen die Druckresultierenden der beiden seitlichen Schubwände die gleiche Neigung auf, was in (8.1) schon berücksichtigt wurde. Einsetzen von (8.3) und (8.2) in (8.1) ergibt die für positives Biegemoment gültige Interaktionsbeziehung

$M=2 Z_{f} h-\frac{T^{2}}{4 F_{0}}\left(\frac{h}{b} \frac{1}{P_{s}}+\frac{1}{P_{u}}\right), \quad M \geq 0$.

Mit den restlichen, relevanten Gleichgewichtsbedingungen verifiziert man erstens, dess die Stahlspannungen in der oberen Schubwand an oder unterhalb der Fliessgrenze liegen, und zweitens, dass mit dem pro Schubwand konstanten Betonspannungszustand überhaupt alle Gleichgewichtsbedingungen erfüllbar sind (siehe Abschnitt 8.5). Solange der Betonspannungszustand wie vorausgesetzt stabil ist, stellt (8.4) einen unteren Grenzwert für die Biege-Torsionsinteraktion des betrachteten, dünnwandigen Rechteckquerschnittes dar, dessen einzelne Scheiben den Fliessbedingungen (2.7) gehorchen.

Diese Aussage bedarf jedoch der Präzisierung. Die Klassifikation einer Querschnittsinteraktionsbeziehung als unterer Grenzwert, oberer Grenzwert oder exakte Lösung kann nur in bezug auf einen fest definierten Satz von verallgemeinerten Spannungen und Verformungen Gültigkeit haben. Mit der Wahl der verallgemeinerten Verformungen oder der verallgemeinerten Spannungen werden explizit oder implizit die Verformungsfreiheitsgrade des Querschnittes festgelegt, da nur einer der beiden Sätze frei wählbar ist, wenn die Dissipationsleistung des Querschnittes als Skalarprodukt der verallgemeinerten Spannungen und Verformungen ausdrückbar sein soll (siehe Abschnitt 6.3). Der Torsionswiderstand eines Querschnittes ist wie die Schubtragfähigkeit (siehe Abschnitte 6.3, 6.4) immer auch von den Bedingungen in benachbarten Querschnitten abhängig. Mit der Definition der verallgemeinerten Grössen werden diese Bedingungen festgelegt. Es ist offensichtlich, dass die Traglast eines torsionsbeanspruchten Balkens davon abhängt, ob sich seine Querschnitte unbehindert verwölben können oder nicht.

Im besonderen wirft der "Skew Bending"-Mechanismus, der tiefere Traglastfaktoren liefert als (8.4), die Frage auf, mit welchen Querschnittsverformungen der Spannungszustand des Fachwerkmodells verträglich ist. In [18, 20] werden kinematische Betrachtungen an einem diskreten Fachwerk (siehe auch Fig. 6.2 b) mit der Balkenannahme "Querschnitte normal zur Balkenaxe 
bleiben eben" (Fig. 8.1 d) kombiniert. Die Geschwindigkeiten $\dot{u}_{k}, \dot{v}_{k}$ in der Ebene der Scheibe $k$ sind dann im allgemeinsten Fall gegeben durch (Fig. $8.1 \mathrm{c}, \mathrm{d}$ )

$\left.\begin{array}{l}\dot{u}_{k}=\dot{u}-z \dot{w}, x-y \dot{v}, x \\ \dot{v}_{k}=-(\dot{v}+\dot{\bar{v}}) \sin \delta+(\dot{w}+\dot{\bar{w}}) \cos +r \dot{\varphi},\end{array}\right\}$

wobei $\dot{u}, \dot{v}, \dot{w}, \dot{\bar{v}}, \dot{\bar{w}}, \dot{\varphi}$ die Verformungsparameter der Balkenaxe sind und ( ), , ( ), Ableitungen in $x-b z w$. s-Richtung bedeuten. Für die

$$
\left.\begin{array}{l}
\dot{\varepsilon}_{x}=\dot{u}_{k, x}=\dot{u}_{, x}-z \dot{w}, x x-y \dot{v}_{, x x}, \\
\dot{r}_{x s}=\dot{u}_{k, s}+\dot{v}_{k, x}=-\dot{\bar{v}}_{, x} \sin \delta+\dot{\bar{w}}_{, x} \cos \delta+r \dot{\varphi}, x,
\end{array}\right\}
$$

Die Grössen (8.8) sind die verallgemeinerten Verformungen des Querschnittes, wobei die den Querkräften assoziierten Verformungen (8.8b) hier nicht interessieren. Für die Gewinnung der Definition der zugehörigen verallgemeinerten Spannungen sei auf Abschnitt 6.3 verwiesen.

In Abschnitt 6 ist ausführlich erläutert, dass das Konzept Makromodell "Diskretes Fachwerk" (Fig. 6.1) aus kinematischer Sicht genau dann nicht mehr haltbar wird, wenn im diskreten Fachwerk die Geometrie variabel eingeführt wird, indem man beim statischen Vorgehen bezüglich der Diagonalenneigung optimiert. Aus der Sicht der normalen Ableitung einer Balkenfliessbedingung andererseits ist zweierlei festzustellen. Erstens sind die Dehnungsgeschwindigkeiten (8.7) mit dem pro Querschnittsseite konstanten Betonspannungszustand nicht verträglich. Da $\dot{\gamma}_{x s}$ einen konstanten, $\dot{\varepsilon}_{x}$ im allgemeinen aber einen linearen Verlauf über eine Querschnittsseite aufweist, muss wegen (6.5a) auch tan 1 linear variieren. Durch Einsetzen von (8.7) in den Ausdruck für die Dissipationsleistung des Querschnittes folgt zweitens, dass die verallgemeinerte Spannung "Torsionsmoment" durch die Beziehung $T$ = $\$ S r$ ds mit dem Schubfluss verknüpft ist. Die "exakte" Interaktionsbeziehung für einen Querschnitt, der sich gemäss (B.5) nicht verwölben kann, erhält man deshalb über die statische Methode im allgemeinen nur, wenn auch bezüglich des Schubflussverlaufs über den Querschnittsumfang optimiert wird. Für einen solchen Querschnitt liefert (8.4) nur untere Grenzwerte und stellt nicht - wie in [18] angenommen - die plastizitätstheoretisch exakte Lösung dar. Dies wird in Abschnitt 8.5.2 bestätigt, wo gezeigt wird, dass bei behinderter Verwölbung im allgemeinen die exakte Traglast höher liegt und für einen nichtkonstanten Schubfluss erreicht wird.

Die Resultate der Abschnitte 6.3 und 6.4 lassen vermuten - und dies wird in Abschnitt 8.4 auch bestätigt - , dass der "Fachwerkmodell"-Spannungszustand mit einem Verformungsansatz verträglich ist, der die freie Verwälbung des Querschnittes zulässt. Wenn diese freie Verwölbung möglich ist, ohne dass kinematische Kompatibilitätsbedingungen zwischen benachbarten 

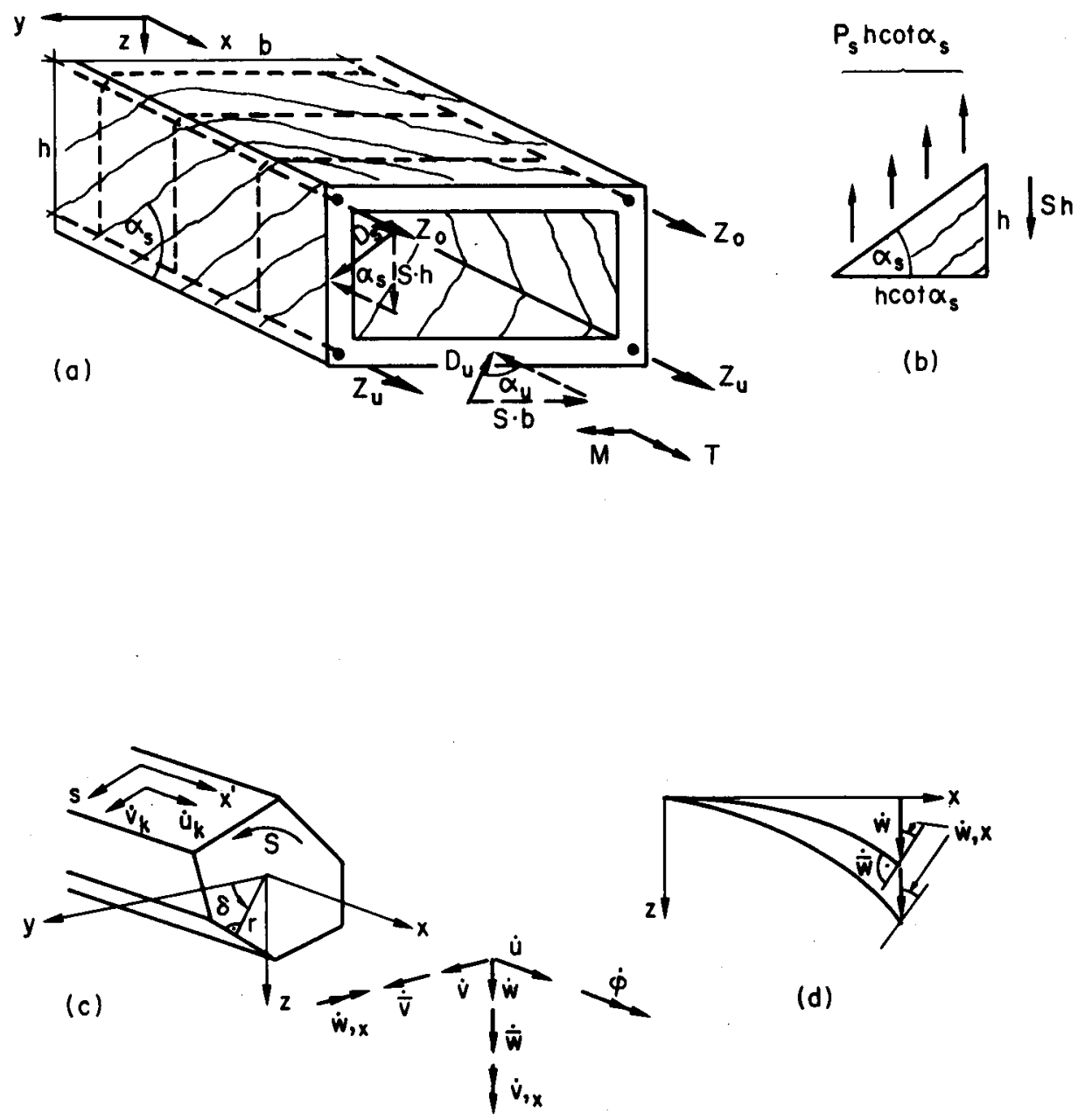

Fig. 8.1 "Fachwerkmodell" noch [18] 
Querschnitten verletzt werden, kann erst beurteilt werden, wenn ein räumlicher, mit dem "Fachwerkmode11"-Spannungszustand verträglicher Mechanismus bekannt ist.

Ein seit langem bekannter, räumlicher Torsionsmechanismus ist der "Skew Bending"-Mechanismus [17]. Er wird im folgenden ebenfalls nur für dünnwandige, geschlossene Querschnitte behandelt (Fig. 8.2). Auch hier wird vorausgesetzt, dass der Beton im Kollapszustand keine Zugspannungen überträgt, und dass der Beton nicht versagt, bevor der Kollaps durch Fliessen eines Teils der Bügel- und Längsermierung eingeleitet wird. Damit wird ebenfalls implizit eine Plastifizierung der einzelnen Scheiben im Fliessregime I der Fliessfigur 2.6 angenommen.

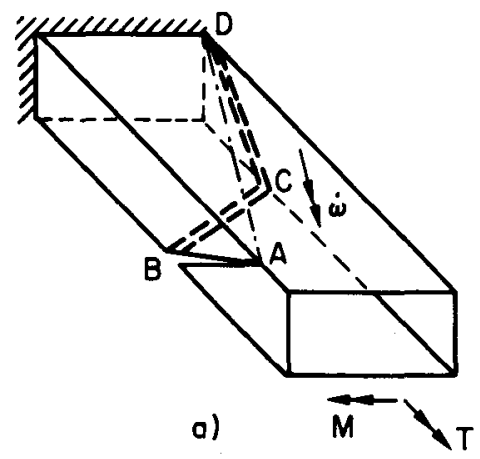

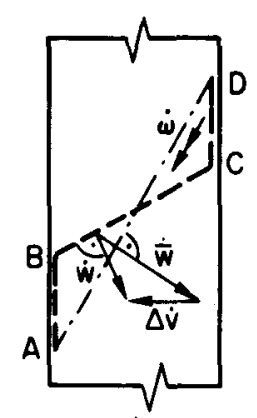

b)

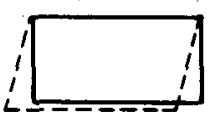

$\overline{\Delta i}$

Fig. 8.2 "Skew Bending"-Mechanismus

Wie schon der Name ausdrückt, wird beim "Skew Bending"-Mechanismus eine starre Rotation des freien Endes des Kragträgers in Fig. 8.2 um eine schiefe Achse AD betrachtet. Dis Lage der Rotationsachse wird dabei durch die beiden Enden eines sich öffnenden, umlaufenden "Risses". ABCD festgelegt. Die Tatsache, dass sich aus diesem Mechanismus tiefere Traglastfaktoren ergeben als aus (8.4), wurde in [27] fälschlicherweise durch den aus der Plastizitätstheorie der Stahlbetonplatte bekannten Begriff der "nodal forces" erklärt. Dabei wurde impliziert, dass die Fachwerkmodellösung im Sinne der "equilibrium method" der Plattentheorie als Mechanismusmethode zu betrachten sei.

Nun ändert jedoch - weil das freie Ende unverformt rotiert - der Traglastfaktor nicht, wenn angenommen wird, dass Biege- und Torsionsmoment am Trägerende über einen starren Endblock eingeleitet werden. In diesem Fall ist aber der Spannungszustand gemäss Fachwerkmodell auch aus der Sicht der Raumstruktur statisch zulässig und stabil und muss zu einem unteren Grenzwert führen.

Der wahre Grund für den Widerspruch ist sofort aus der Aufsicht in Fig. 8.2 b ersichtlich. Wie schon in Abschnitt 3.3 erwähnt, ist der "Riss" ABCD innerhalb der Plastizitätstheorie als Unstetigkeitslinie des Geschwindigkeitsfeldes zu betrachten. Rotiert das freie Trägerende wie angenommen starr, und bleibt somit ausserhalb des unmittelbaren Mechanismusbereichs ABCD insbesondere die Querschnittsform erhalten, so springt längs BC nicht nur die zu BC normale, sondern auch die tangentiale Komponente der Geschwindigkeit in Scheibenebene. Solange die Betonfliessfigur durch Fig. 2.1 oder Fig. 4.3 b gegeben ist, muss deshalb bei der Berechnung der Dissipationsleistung der Anteil infolge Betonstauchens ebenfalls berücksichtigt werden. Damit würde aber der "Skew Bending"-Mechanismus höhere Traglastfaktoren liefern als das Fachwerkmodell, solange in letzterem der Betonspannungszustand stabil ist.

Es ist schon in Abschnitt 3.3 darauf hingewiesen worden, dass die Nichtberücksichtigung der Betondissipationsleistung der Verwendung der Coulomb-Fiessbedingung für den ebenen Dehnungszustand als Betonfliessfigur entspricht (Fig. 3.7). Mit dem Winkel zwischen $\bar{w}$ und $B C$ 
(Fig. 8.2 b) wäre dann auch der Winkel der inneren Reibung $\phi$ van der Armierung und der Beanspruchung abhängig. Auch aus dieser Sicht ergibt sich somit keine sinnvolle Interpretation für die Vernachlässigung der Betondissipationsleistung.

Die kinematische Restriktion (3.15) des Fliessregimes I (senkrechte kollapsrissöffnung) kann entlang der Unstetigkeitslinie ABCD offensichtlich nur dann erfüllt werden, wenn im freien Kragarmende eine Querschnittsverformung $\Delta \dot{v}$ zugelassen wird (Fig. 8.2 b,c). Die in der einfachen Balkentheorie grundlegende Voraussetzung der Querschnittsformerhaltung kann offensichtlich nicht gleichzeitig mit (3.15) erfüllt werden, solange man sich auf die Betrachtung einer einzigen, zusammenhängenden Unstetigkeitslinie beschränkt. Für den als Faltwerk aufgefassten Kragarm dagegen stellt der Mechanismus mit $\Delta \dot{v}$ lediglich eine spezielle, im allgemeinen nicht ungünstigste Kombination der in Fig. 8.3 angedeuteten Faltwerkmechanismen dar, bei welchen die Querschnitte vor dem Kollapsriss ǹicht nur die Querschnittsform ändern, sondern auch wölben. Die Traglast des Faltwerks kann deshalb nicht unabhängig von der Randspannungsverteilung sein, über die das Biege- und Torsionsmoment am freien Ende eingeleitet werden.

Die Faltwerkstheorie liefert immer tiefere oder höchstens gleich hohe Traglasten als die Balkentheorie, da beim Faltwerk die mit den restriktiveren Verformungsannahmen der Balkentheorie verbundenen, internen Reaktionen verschwinden müssen. Dies lässt sich mit den Mechanismen in Fig. 8.3 leicht nachweisen.

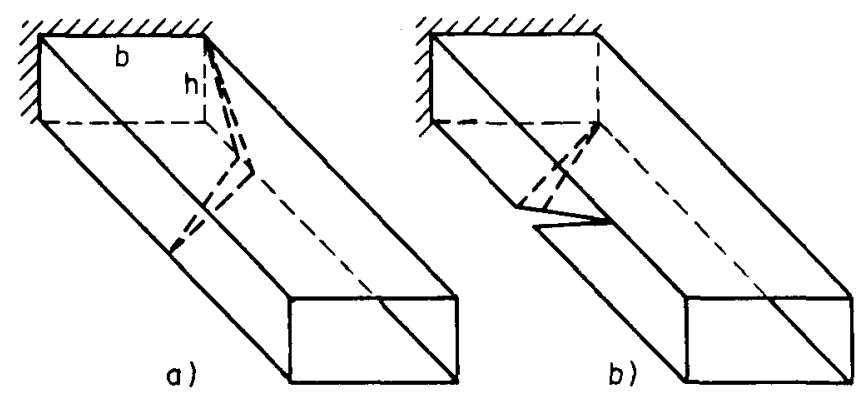

Fig. 8.3 Grundmechanismen für Faltwerk

Da die beiden Mechanismen von Fig. 8.3 eine entgegengesetzte Querschnittsverformung zur Folge haben, lassen sie sich jedoch unter Verzicht auf die zusammenhängende Unstetigkeitslinie auch so miteinander kombinieren, dass die Querschnitte ausserhalb des Kollapsrissbereichs nicht nur wie beim "Skew Bending"-Mechanismus eben bleiben, sondern auch ihre Form nicht ändern. Dieser Mechanismus wird im folgenden Abschnitt behandelt und ergibt wieder die Interaktionsbeziehung $(8.4)$. 
Dem in Fig. 8.4 dargestellten Mechanismus mit diskontinuierlichem Geschwindigkeitsfeld liegen folgende Voraussetzungen zugrunde:

1. Es wird nur die Membranwirkung der Schubwände berücksichtigt.

2. Die Schubwände sind im Fliessregime I plastifiziert.

3. Die Endquerschnitte des Balkens bleiben eben und ihre Querschnittsform bleibt erhalten.

Aufgrund der ersten Voraussetzung werden Spannungs- und Verformungszustand der Schubwände durch die Membrankräfte $N_{x}, N_{y}, N_{x y}$ und die Geschwindigkeiten. $\dot{u}, \dot{v}$ in Wandebene beschrieben. Als Unstetigkeiten des Geschwindigkeitsfeldes kommen gemäss der zweiten Voraussetzung nur sich senkrecht öffnende Kollapsrisse (Abschnitt 3.3) in Frage, die nach Abschnitt 9 Geraden sein müssen. Die Zulässigkeit der zur Wandebene normalen Komponente der Geschwindigkeitssprünge wird in Abschnitt 8.5.4 nachgewiesen. Bezüglich der dritten Voraussetzung ist zu bemerken, dass das Ebenbleiben der Endquerschnitte bei genügend langen Balken die Verwölbung der Feldquerschnitte nicht behindert. Auf die Bedeutung dieser dritten Voraussetzung wird später noch ausführlich eingegangen.

Es wird wieder eine starre Rotation der vorderen Balkenhälfte um eine z.B. in der oberen Scheibe liegende schiefe Achse AE betrachtet. Die Neigung $B$ der Rotationsachse ist jedoch nicht wie in Fig. 8.2 durch die Enden eines "Risses" bestimmt, sondern wird als zusätzlicher Parameter eingeführt, der sich aus (3.15) und der dritten Annahme bestimmt.

Folglich sind zwei, von den Punkten $A$ und $E$ ausgehende Kollapsrisse zu betrachten. Der Knick entlang $A E$ öffnet in der vorderen Scheibe den Kollapsriss $A B$, der seinerseits in der unteren
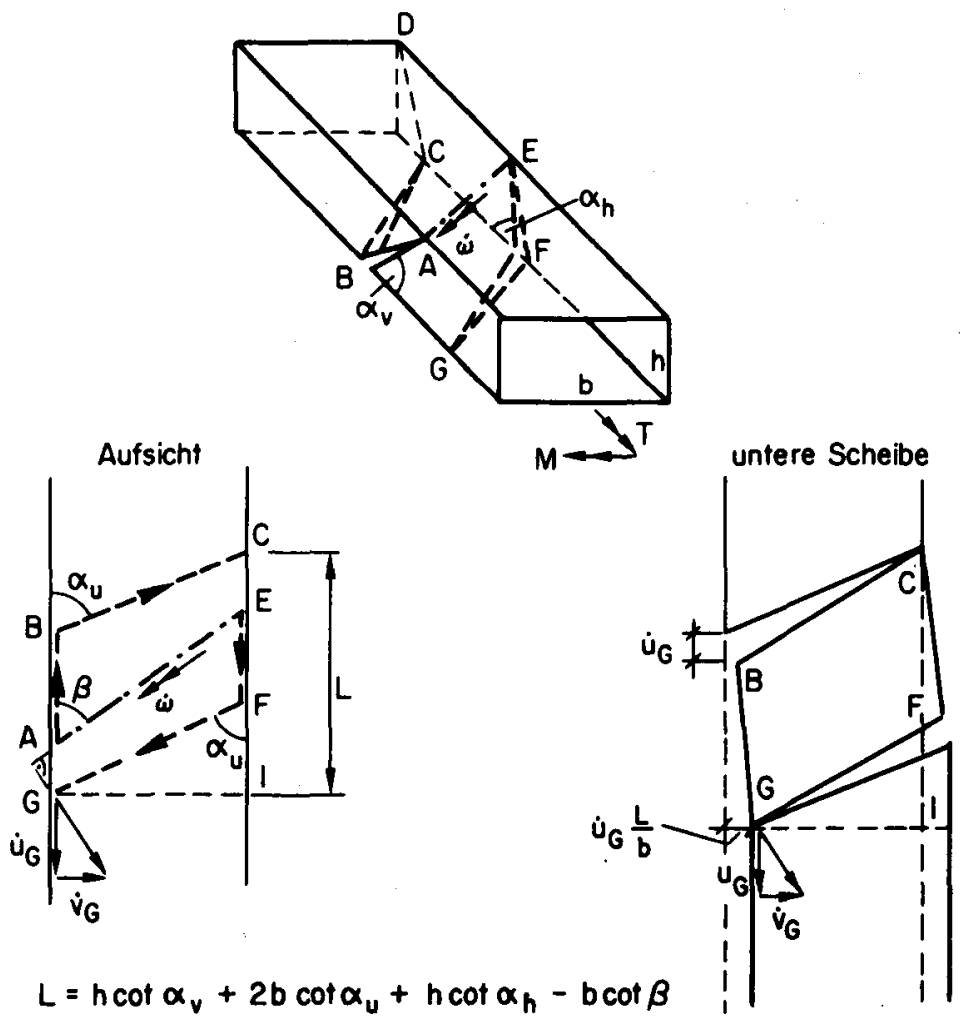

Fig. 8.4 Grundmechanismus für Balken mit Rechteckquerschnitł 
Scheibe den Kollapsriss BC öffnet. In C ist der Kollapsriss wieder geschlossen, da in D die obere Scheibe keinen Knick aufweist. Gleiches gilt für den Kollapsriss EFG.

Die beiden umlaufenden Kollapsrisse trennen aus der unteren Scheibe das Parallelogramm BCFG, das entlang den Kanten CF bzw. BG mit der ruhenden bzw. rotierenden Balkenhälfte verbunden ist. Da die Kanten mit Ausnahme der Punkte $B$ und F ungedehnt bleiben, kann das Stück BCFG unter der Voraussetzung (3.15) in Scheibenebene nur eine Starrkörperrotation erleiden.

Genügen die Endquerschnitte der Annahme 3 , so müssen die Trägerhälften hinter ABCE und vor AEFG starr bleiben bzw. starr rotieren. Die Geschwindigkeiten des Punktes $G$ in der Ebene der unteren Scheibe ergeben sich somit zu (Fig. 8.4 Aufsicht)

$\left.\begin{array}{l}\dot{u}_{G}=\dot{\omega h} \sin \beta \\ \dot{v}_{G}=\dot{\omega h} \cos \beta\end{array}\right\}$

Betrachtet man andererseits die Starrkörperrotation in Scheibenebene des Parallelogramms BCFG (Fig. 8.4 untere Scheibe), so folgt

$\dot{v}_{G}=\dot{u}_{G} \frac{L}{b}$,

wobei L gemäss Fig. 8.4 Funktion der Neigung $\beta$ der Rotationsachse und der freien Parameter des Mechanismus, d.h. der Kollapsrissneigungen a,ist.

$L=h \cot \alpha_{v}+2 b \cot \alpha_{u}+h \cot \alpha_{h}-b \cot \beta$.

Einsetzen von $(8.9)$ und $(8.11)$ in (8.10) ergibt

$\cot \beta=\frac{L}{b}=\frac{1}{2}\left(\cot \alpha_{v}+\cot \alpha_{h}\right) \frac{h}{b}+\cot \alpha_{u}$.

Der Mechanismus erfüllt die Bedingung (3.15) überall, und der Balken bleibt ausserhalb des Kollapsrissbereichs für beliebige Kollapsrissneigungen starr, wenn das Verhältnis cotß von Verdrehung und Biegerotation entsprechend (8.12) gewählt wird.

Für das in Abschnitt 8.1 behandelte Beispiel sind äussere Leistung La und Dissipationsleistung L gegeben durch:

$L_{a}=\dot{\omega} \sin \beta M+\dot{\omega} \cos \beta T$,

$L_{d}=\dot{\omega} \sin \beta\left\{\frac{1}{2} h^{2}\left(\cot ^{2} \alpha_{v}+\cot ^{2} \alpha_{h}\right) P_{s}+b h \cot ^{2} \alpha_{u} P_{u}+2 z_{f} h\right\}$.

Gleichsetzen von (8.13) und (8.14) unter Beachtung von (8.12) ergibt

$$
\begin{aligned}
M=2 Z_{f} h & +\frac{1}{2}\left(h^{2} P_{s} \cot ^{2} \alpha_{v}-\frac{h}{b} T \cot \alpha_{v}\right) \\
& +\frac{1}{2}\left(h^{2} P_{s} \cot ^{2} \alpha_{h}-\frac{h}{b} T \cot \alpha_{h}\right) \\
& +\frac{h}{h}\left(h^{2} P_{u} \cot ^{2} \alpha_{u}-\frac{h}{b} T \cot \alpha_{u}\right) .
\end{aligned}
$$


Durch Minimieren von M für festes $T$ bezüglich der Kollapsrissneigungen erhält man

$$
\begin{aligned}
& \frac{\partial M}{\partial\left(\cot \alpha_{v}\right)}=h^{2} P_{s} \cot \alpha_{v}-\frac{1}{2} \frac{h}{b} T=0, \\
& \cot \alpha_{v}=\frac{T}{2 b h} \frac{1}{P_{s}}=\frac{T}{2 F_{0}} \frac{1}{P_{s}}=\frac{s}{P_{s}},
\end{aligned}
$$

und entsprechend folgt

$\cot \alpha_{h}=\cot \alpha_{v}=\frac{S}{P_{s}}, \quad \cot \alpha_{u}=\frac{S}{P_{u}}$.

Durch Einsetzen von (8.16) in (8.15) findet man schliesslich wieder die Interaktionsbeziehung (8.4).

$M=2 Z_{f} h-\frac{T^{2}}{4 F_{0}}\left(\frac{h}{b} \frac{1}{P_{s}}+\frac{1}{P_{u}}\right)$.

Die Gesamtheit der Interaktionsbeziehungen gewinnt man, indem man auch die riechanismen mit Rotationsachse in den übrigen drei Scheiben untersucht. Aufgrund der vorhandenen Symmetrien ergibt sich natürlich

$$
|M|=2 Z_{f} h-\frac{T^{2}}{4 F_{0}}\left(\frac{h}{b} \frac{1}{P_{s}}+\frac{1}{P_{u}}\right)
$$

Für reine Torsion fliesst im Kollapszustand, ebenfalls aus Symmetriegründen, die gesamte Armierung. Als Kollapsmechanismen kommen deshalb sowohl alle vier Grundmechanismen als auch Kombinationen von ihnen in Frage. Insbesondere ist auch ein Mechanismus mit reiner Verdrehung und Verlängerung möglich, wie ihn Fig. 8.5 zeigt. Er kann als Linearkombination mit positiven Koeffizienten von zwei Grundmechanismen mit Rotationsachse in der oberen bzw. unteren Scheibe aufgefasst werden.

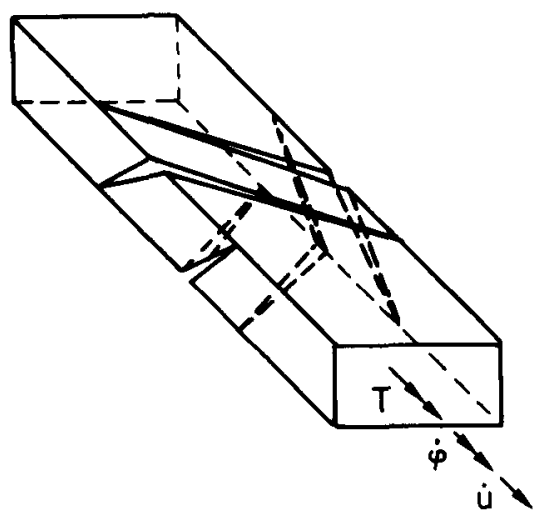

\section{Fig. 8.5 Kombinierter Mechanismus für Bolken}

Aus den Ergebnissen dieses Abschnittes geht hervor, dass die beiden Grundideen zur Erfassung des Kollapszustandes von Stahlbetonträgern unter Torsion und Biegung, nämlich der fachwerkartige Spannungszustand einerseits und der Mechanismus mit einer schiefen, in einer Scheibe gelegenen Rotationsachse andererseits, sich nicht widersprechen. Vielmehr ergänzen sich die beiden Ansätze unter noch zu präzisierenden Verformungsannahmen der Balkentheorie zur plastizitätstheoretisch exakten Lösung, wenn bei der Mechanismusmethode die kinematische Restriktion (3.15) des Fliessregimes I nicht verletzt wird. 
Ein stabiler Spannungszustand gemäss Fachwerkmodell ist statisch zulässig und liefert damit untere Grenzwerte für die Traglast, wenn die statischen Randbedingungen an den Trägerenden lediglich integral über den Querschnitt, d.h. In den Schnittkräften, erfüllt zu werden brauchen. Die duale, kinematische Interpretation dieser Balkenannahme ist das Ebenbleiben und die Formerhaltung der End- bzw. Lasteinleitungsquerschnitte. Die äussere Leistung lässt sich daher durch $M$ und $T$ ausdrücken und ist unabhängig von der Randspannungsverteilung, über die Biege - und Torsionsmoment eingeleitet werden.

Die neuen Mechanismen gemäss Fig. 8.4 liefern unter dieser Verformungsannahme obere Grenzwerte für die Traglast, wenn sie kinematisch zulässig sind, d.h. wenn die Kollapsrissneigungen so gewählt werden, dass die Ausdehnung des Mechanismus die Balkenlänge nicht übertrifft. Ist der Balken lang genug, dass sich unbehindert der Mechanismus mit den zum kleinsten oberen Grenzwert führenden Kollapsrissneigungen ausbilden kann, so sind - wie das behandelte Beispiel zeigt - die Geschwindigkeitsfelder des massgebenden Mechanismus verträglich mit den Membrankräften gemäss Fachwerkmodell: Die Rotationsachse des Mechanismus liegt in der Scheibe mit der nicht massgebenden Armierung, und in den übrigen Scheiben stimmen die Kollapsrissneigungen (8.16) mit den Neigungen (8.3) der Betondrucktrajektorien überein.

Die Interaktionsbeziehungen ( 8.4$)$ bzw. (8.17) liefern somit die exakte Traglast aufgrund der Fliessbedingung $(2.7 \mathrm{I})$, wenn als Verformungsannahmen die Formerhaltung und das Ebenbleiben der End- oder Lasteinleitungsquerschnitte und die unbehinderte Längsausdehnung des Mechanismus eingeführt werden. Wird zudem nachgewiesen, dass die Membrankräfte im Beton stabil sind, so gilt das Gesagte natürlich auch für die Gesamtheit der Fliessbedingungen (2.7).

Die obigen Verformungsannahmen sind etwas ungewohnt, weil statt der Verzerrungsgeschwindigkeiten eines vollplastifizierten Querschnittes ein räumlicher Mechanismus mit diskontinuierlichem Geschwindigkeitsfeld und endlicher Ausdehnung in Balkenlängsrichtung betrachtet wird. Wie in Abschnitt 8.4 gezeigt wird, entsprechen jedoch diese Annahmen dem in der Balkentheorie im Zusammenhang mit einem konstanten Torsionsmoment üblichen Ansatz für die Querschnittsverformung. Das Ebenbleiben der Endquerschnitte verlangt, dass die Summe der Kollapsrissweiten in Balkenlängsrichtung eben über den Querschnitt verteilt ist, und entspricht der Annahme einer ebenen Verteilung der Dehnungsgeschwindigkeiten $\dot{\varepsilon}_{x}$ im vollplastifizierten Querschnitt. Das Ebenbleiben der Endquerschnitte behindert aber bei genügender Balkenlänge nicht die Verwölbung der Feldquerschnitte. Die Tatsache, dass die Grenze zwischen starren und im Regime I fliessenden Zonen nicht entlang einem zur Balkenachse senkrechten Querschnitt verlaufen kann. bedeutet im Gegenteil, dass ein vollplastifizierter Querschnitt wölben muss. Die Voraussetzung, dass sich der Kollapsmechanismus in Balkenlängsrichtung unbehindert ausdehnen kann, entspricht somit der Annahme einer unbehinderten Verwölbung des vollplastifizierten Querschnittes.

\subsection{Kollapsrissmechanismus für Balken mit polygonalem Querschnitt}

Der Mechanismus von Fig. 8.4 lässt sich sofort auch auf Balken mit dünnwandigen, geschlossenen Querschnitten von konvexer, polygonaler Form verallgemeinern (Fig. 8.6). Die Annahmen bleiben die gleichen wie im vorangehenden Abschnitt. Der Mechanismus gemäss Fig. B.6 ist mit den Membrankräften gemäss Fachwerkmodell verträglich, wenn die Rotationsachse in die Scheibe mit der nicht massgebenden Armierung gelegt wird, für die Kollapsrissneigungen die aus der Fachwerkmodelllösung asonnenen Neigungen der Betondrucktrajektorien eingesetzt werden, und wenn die Bedingung (3.15) nicht verletzt wird. Die mit dem Fachwerkmodell gewonnenen Interaktionsbeziehungen liefern für genügend lange Balken die exakte Traglast aufgrund der Fliessbedingung ( $2.7 \mathrm{I})$, wenn der Mechanismus auch kinematisch zulässig ist. Es ist 5o- 


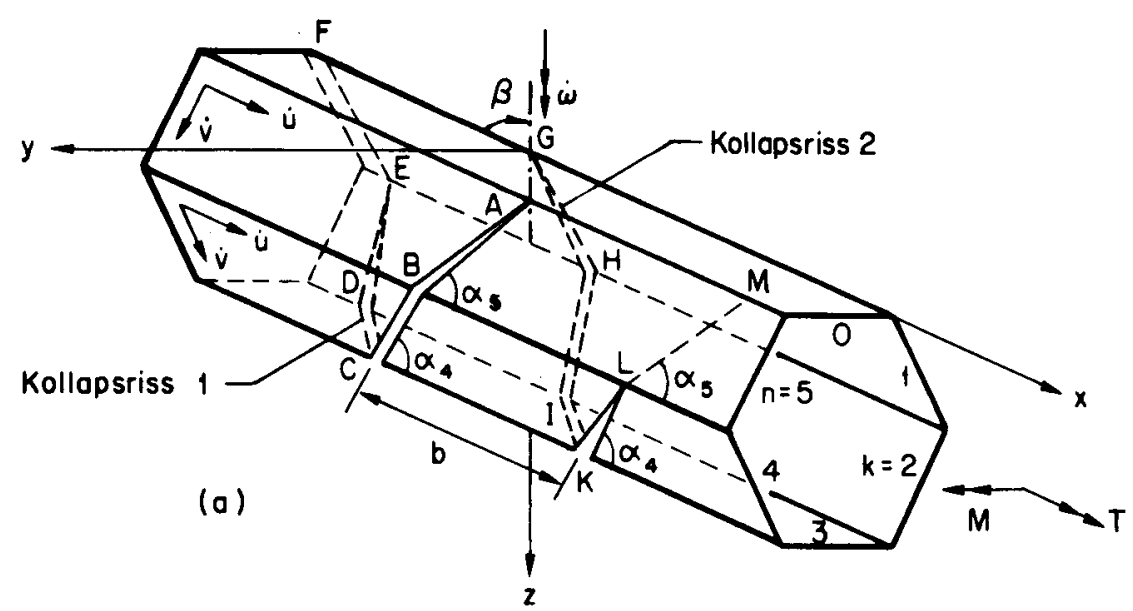

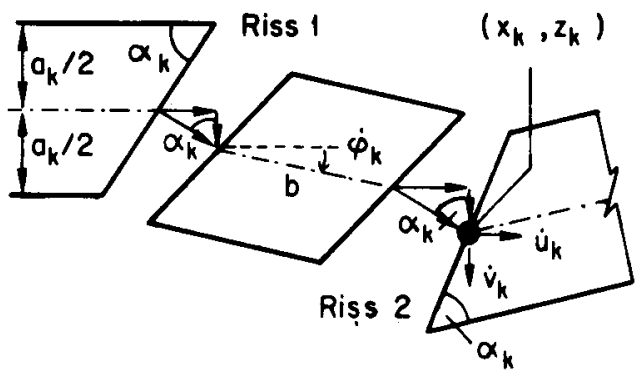

(b)

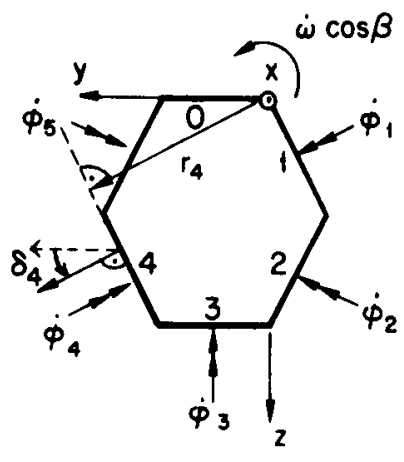

(c)

Fig. 8.6 Grundmechonismus für Balken mit polygonalem Querschnitt

mit noch zu zeigen, wie die Neigung $\beta$ der Rotationsachse zu wählen ist, damit unter Einhaltung von (3.15) für die End-bzw. Lasteinleitungsquerschnitte die Bedingungen der Formerhaltung und des Ebenbleibens erfüllt werden können.

Rotiert der vordere Teil des Balkens von Fig. 8.6 a starr um die schiefe Achse $A G$, so trennen die beiden sich öffnenden Kollapsrisse ABCDE und GHIKL aus dem Balken einen umlaufenden Streifen BCDEHIKL der Breite $b$, der nur entlang den Kanten EH und BL mit dem ruhenden bzw. rotierenden Balkenteil verbunden ist.

Denkt man sich die beiden Kollapsrisse bis $F$ bzw. M fortgesetzt, obschon sie in Wirklichkeit längs EF bzw. LM geschlossen sind, und betrachtet man nun den von FG bis AM umlaufenden Streifen zwischen den zwei Kollapsrissen, so muss offensichtlich an den beiden Streifenenden FG und AM die Geschwindigkeitskomponente in Balkenlängsrichtung verschwinden. Bezeichnet $\varphi_{k}$ die Starrkörperrotation in Scheibenebene des Streifenstücks in der Scheibe $k$ (Fig. 8.6 b,c) und $a_{k}$ die Breite der Scheibe $k$, so muss folglich gelten

$\sum_{k=1}^{n} \dot{\varphi}_{k} a_{k}=0$.

Die Konvention für die Scheibennumerierung ist Fig. 8.6 zu entnehmen. 
Rotiert der Balkenteil vor GHIKLA starr, so gilt für die Geschwindigkeiten in Scheibenebene $\dot{u}_{k}, \dot{v}_{k}$ des Punktes $\left(x_{k}, z_{k}\right)$, der auf dem vorderen Rissufer des kollapsrisses 2 in der Scheibe $k$ liegt (Fig. $8.6 \mathrm{a}, \mathrm{b}, \mathrm{c})$ :

$\left.\begin{array}{l}\dot{u}_{k}=\dot{\omega} \sin \beta z_{k}, \\ \dot{v}_{k}=\dot{\omega} \cos \beta r_{k}-\dot{\omega} \sin \beta x_{k} \cos \delta_{k} .\end{array}\right\}$

Aus Fig. 8.6 b liest man andererseits ab

$\dot{v}_{k}=\dot{u}_{k} \cot \alpha_{k}+\dot{\varphi}_{k} b$,

wenn beachtet wird, dass sich die beiden Kollapsrisse gemäss (3.15) senkrecht zur Rissrichtung öffnen müssen. Für die Rotation $\dot{\varphi}_{k}$ folgt somit aus $(8,19)$ und $(8.20)$

$\dot{\varphi}_{k}=\frac{\dot{\omega} \sin \beta}{b}\left\{r_{k} \cot \beta-\left(z_{k} \cot \alpha_{k}+x_{k} \cos \delta_{k}\right)\right\}$.

Als Kontrolle verifiziert man durch Einsetzen der entsprechenden Werte für die Scheiben 1 und 5 (Fig. 8.6) leicht, dass $\dot{\varphi}_{1}=0$ und $\dot{\varphi}_{5}$ gleich der Projektion des Vektors $\dot{\omega}$ auf die Innennormale der Scheibe 5 ist.

Einsetzen von (8.21) in (8.18) ergibt die gesuchte Beziehung für die Neigung $\beta$ der Rotationsachse

$\cot \beta=\frac{1}{2 F_{0}}\left\{\sum_{k} z_{k} a_{k} \cot \alpha_{k}+\sum_{k} x_{k} a_{k} \cos \delta_{k}\right\}$

Dabei darf über alle Scheiben summiert werden, da für die Scheibe 0 , in der die Rotationsachse liegt, die Grössen $r_{k}, z_{k}$, cos $\delta_{k}$ verschwinden. $F_{0}$ bezeichnet die vom Querschnitt umschlossene Fläche. Beachtet man noch, dass der Ausdruck in der geschweiften Klammer von (8.22) die doppelte Fläche zwischen dem in die $x$-z-Ebene projektierten Kollapsriss GHIKLM und der $x$-Achse darstellt, so lässt sich (8.22) auch in der einfacheren Form

$\left\|\cot \beta=\frac{1}{F_{0}} \sum_{k} z_{k} a_{k} \cot \alpha_{k}\right\|$

schreiben, wenn sich $z_{k}$, wie in Fig. 8.6 b dargestellt, auf Punkte der Scheibenmittellinie bezieht. Man verifiziert leicht, dass für den Balken mit Rechteckquerschnitt die Formeln (8.23) und $(8.12)$ identisch sind.

Da in die Ableitung der Gleichung (8.23) nur die Summe $\dot{u}_{k}$ der Kollapsissweiten in Balkenlängsrichtung eingegangen ist (Fig. 8.6 b), ist im konkreten Fall noch zu zeigen, dass sich tatsächlich beide kollapsrisse überall öffnen.

Gleichung (8.23) wird im folgenden Abschnitt 8.4 ebenfalls aus einer reinen Querschnittsbetrachtung abgeleitet, und die darauf aufbauenden, mit der Fachwerkmodellösung identischen Interaktionsbeziehungen werden dort berechnet. 
Die Berechnung der Traglast von als Balken idealisierten Trägern aufgrund räumlicher Geschwindigkeits- und Spannungsfelder wie in den vorangehenden Abschnitten ist nicht das übliche Vorgehen. Der theoretische Uebergang von der dreidimensionalen Tragstruktur zum eindimensionalen Balken erfolgt normalerweise auf der Stufe einer reinen Querschnittsbetrachtung. Aufgrund von Verformungsansätzen für den vollplastifizierten Querschnitt werden Querschnittsinteraktionsbeziehungen, d.h. Fliessbedingungen für das differentielle Balkenelement, gewonnen. Das Traglastproblem wird dann nur noch in den durch den Verformungsansatz definierten verallgemeinerten Spannungen und Verformungen formuliert (siehe Abschnitt 6.3).

In diesem Abschnitt soll gezeigt werden, dass die Interaktionsbeziehungen, die mit dem Membranspannungszustand gemäss Fachwerkmodell und den Mechanismen gemäss Figuren 8.4, 8.6 gewonnen werden, die plastizitätstheoretisch exakte Querschnittsinteraktion aufgrund der Fliessbedingung ( 2.7 I) darstellen, wenn von einem Verformungsansatz ausgegangen wird, der eine ebene Verteilung der Längsdehnungsgeschwindigkeiten und die unbehinderte Verwölbung des Querschnittes postuliert. Dabei wird selbstverständlich auch Querschnittsformerhaltung vorausgesetzt.

Der Ansatz (8.5) ist somit durch einen Wölbterm zu ergänzen (alle folgenden Formeln beziehen sich wieder auf Fig. $8.1 \mathrm{C}$ ).

$\left.\begin{array}{l}\dot{v}_{k}=\dot{u}-z \dot{w}, x-y \dot{v}, x-\omega \dot{\varphi}, x \\ \dot{v}_{k}=-\dot{v} \sin \delta+\dot{w} \cos \delta+\dot{\varphi} .\end{array}\right\}$

Dabei sind die hier nicht interessierenden Parameter $\bar{v}, \bar{w}$ weggelassen worden. $\omega=\omega(s)$ ist die Wölbfunktion und mit dem Term rí wird die Querschnittsformerhaltung postuliert. Mit den verallgemeinerten Verformungen

$$
\begin{aligned}
& \dot{\varepsilon}=\dot{u}_{, x^{\prime}} \dot{x}_{y}=-\dot{w}, x x^{\prime} \dot{x}_{z}=-v, x x^{\prime} \\
& \dot{v}=\dot{\varphi}_{, x^{\prime}} \\
& \dot{\varphi}_{, x x}=\dot{v}_{, x}=0,
\end{aligned}
$$

folgt für die Dehnungsgeschwindigkeiten in Scheibenebene

$$
\begin{aligned}
& \dot{\varepsilon}_{x}=\dot{u}_{k, x}=\dot{\varepsilon}+z \dot{x}_{y}+y \dot{x}_{z}, \\
& \dot{r}_{x s}=\dot{u}_{k, s}+\dot{v}_{k, x}=(r-\omega, s) \dot{v} .
\end{aligned}
$$

Da der Querschnitt geschlossen ist, muss gelten

$$
\begin{aligned}
& \dot{\oint} \phi \dot{w}_{, s} d s=\oint\left(r \dot{v}-\dot{\gamma}_{x s}\right) d s=0, \\
& \dot{v}=\frac{1}{2 F_{0}} \oint \dot{\gamma}_{x s} d s .
\end{aligned}
$$

wobei $F_{0}$ die vom Querschnitt umschlossene Fläche bezeichnet. 
Mit den Gleichungen (8.26), (8.28), die die Verzerrungsgeschwindigkeiten mit den verallgemeinerten Verformungen verknüpfen, sind - wie in Abschnitt 6.3 ausführlich gezeigt wurde auch die Beziehungen festgelegt, die die normalen Spannungen mit den verallgemeinerten Spannungen verknüpfen, da nur ein Satz frei gewählt werden darf. In (8.26) ist der Verlauf der Dehnungsgeschwindigkeit $\dot{\varepsilon}_{x}$ durch die verallgemeinerten Verformungen (8.25a) festgelegt. Ana$\log z u$ den Beziehungen (6.10a) und (6.12a) sind die zugehörigen verallgemeinerten Spannungen $N, M_{y}, M_{z}$ als Integrale definiert. Der Verlauf der Membrankraft $N_{x}$ über den Querschnittsumfang ist nicht festgelegt, sondern gesuchte Funktion.

$N=\oint N_{x} d s, \quad M_{y}=\oint N_{x} z d s, \quad M_{z}=\oint N_{x} y d s$

In (8.27) dagegen ist der Verlauf der Schiebungsgeschwindigkeit $\dot{\gamma}_{x s}$ durch die verallgemeinerte Verformung (8.25b) nicht festgelegt, weil sich der Querschnitt verwölben kann. Vielmehr ist die Verdrehung pro Längeneinheit (8.25b) gemäss (8.28) proportional zur mittleren Schiebungsgeschwindigkeit und damit als Integralgrösse angesetzt. Die Verknüpfung des Schubflusses $S$ mit der verallgemeinerten Spannung Torsionsmoment $T$ ist deshalb analog zu den Beziehungen $(6.10 b)$ und $(6.12 b)$ gegeben durch

$T=2 F_{0} S$.

Statt des Verlaufs der Schiebungsgeschwindigkeiten ist nun der Schubflussverlauf a priori festgelegt und konstant. Die statischen Annahmen des Fachwerkmodells, insbesondere die Voraussetzung eines konstanten Schubflusses, entsprechen genau (8.29) und lassen sich somit auch durch den Querschnittsverformungsansatz $(8.24)$ bis $(8.28)$ begründen.

Da im Fliessregime I die Dehnungsgeschwindigkeiten (8.26) positiv sein müssen, $\dot{\varepsilon}_{x} \geq 0$, muss die Neutralaxe des Mechanismus immer auf dem Querschnittsrand oder ausserhalb des Querschnittes liegen, und die verallgemeinerten Verformungen (8.25a) können nicht beliebig kombiniert werden. Setzt man wie bisher polygonale, konvexe Querschnitte voraus, so gibt es nur eine endliche Anzahl linear unabhängiger Kombinationen von $\dot{\varepsilon}, \dot{x}_{y}, \dot{x}_{z}$, nämlich diejenigen Mechanismen mit $\dot{\varepsilon}_{x} \geq 0$, bei welchen die Neutralachse in einer Querschnittsseite liegt. Jeder dieser Grundmechanismen liefert eine Fliessfläche im ( $, M_{y}, M_{z}$, T)-Raum. Alle übrigen, mit dem Regime I verträglichen Mechanismen sind Kombinationen dieser Grundmechanismen und entsprechen Kanten und Ecken der Fliessfigur.

Im folgenden wird deshalb ein solcher Grundmechanismus betrachtet und die zugehörige Interaktionsbeziehung abgeleitet. Anstelle von (8.25a) wird als veraligemeinerte Verformung die Krümmung $\dot{\bar{X}}$ bezüglich derjenigen Querschnittsseite eingeführt, in der die Neutralachse liegt (Fig. 8.7). Die zugehörige veraligemeinerte Spannung ist das Moment $\bar{M}=f\left(N_{,}, M_{y}, M_{z}\right)$ um die $\bar{y}$-Achse. Die Gleichungen (8.26) und (8.29a) werden somit ersetzt durch

$\dot{\varepsilon}_{x}=\bar{z} \dot{\bar{x}}$,

$\bar{M}=\oint N_{x} \bar{z} d s$.

Das Problem lässt sich damit vorerst im zweidimensionalen Spannungs- und Verformungsraum behandeln. Gleichzeitig werden auch die Zusammenhänge mit den Kollapsrissmechanismen gemäss Figuren 8.4 und 8.6 deutlicher. 
Bezeichnen $P_{x}$ und $P_{s}$ die Fliesskraft pro Längeneinheit der Längs- bzw. Bügelarmierung, so lautet die Fliessbedingung (2.7 I) im x-s-Koordinatensystem der Scheiben (Figuren $8.1 \mathrm{c}$, 8.7)

$N_{x s}^{2} \leq\left(P_{x}-N_{x}\right)\left(P_{S}-N_{s}\right), \quad$ mit $N_{x s} \equiv S$

Die bei einer reinen Querschnittsbetrachtung in den Gleichgewichtsbedingungen nicht enthaltene Membrankraft $N_{s}$ normal zur Balkenachse wird in der Balkentheorie durch Nullsetzen eliminiert

$S^{2}=N_{X S}^{2} \leq\left(P_{X}-N_{x}\right) P_{S} \cdot$

Die Stützfunktion der Fliessfigur (8.32) im $\left(N_{x}, N_{x y}\right)$-Raum ist gegeben durch

$H\left(\dot{\varepsilon}_{x}, \dot{\gamma}_{x s}\right)=L_{d}=P_{x} \dot{\varepsilon}_{x}+P_{s} \frac{\dot{r}_{x s}^{2}}{4 \dot{\varepsilon}_{x}}, \dot{\varepsilon}_{x} \geq 0$,

was man leicht verifiziert, wenn beachtet wird, dass sie mit der spezifischen Dissipationsleistung identisch ist, und dass der Koeffizient von $P_{s}$ wegen (3.8) die Dehnungsgeschwindigkeit $\dot{\varepsilon}_{s}$ der Bügel darstellt.

Die Dissipationsleistung des gesamten Querschnittes ergibt sich mit (8.28), (8.30) und (8.33) zu

$L_{d}^{Q}=\dot{\bar{\chi}} \oint P_{x} \bar{z} d s+\frac{1}{\dot{\bar{x}}} \oint P_{s} \frac{\dot{\gamma}_{x s}^{2}}{4 \bar{z}} d s$,

$\dot{\bar{x}} \geq 0$

$2 F_{0} \dot{\jmath}=\oint \dot{\gamma}_{x s} d s$

Sie ist noch Funktional der Schiebungsgeschwindigkeit $\dot{\gamma}_{x s}$, da deren Verlauf über den Querschnittsumfang durch $\dot{v}$ nicht eindeutig festgelegt ist. Gesucht ist also diejenige Funktion $\dot{\gamma}_{x s}(s)$, die für fest vorgegebene Werte von $\dot{\bar{\chi}}, \dot{\vartheta}$, d.h. unter der Nebenbedingung (8.35b), die Dissipationsleistung (8.34) zu einem Minimum macht. Dies ist ein einfaches Variationsproblem mit Nebenbedingung. Da keine Ableitungen der gesuchten Funktion $\dot{\gamma}_{x s}$ vorkommen, wird die

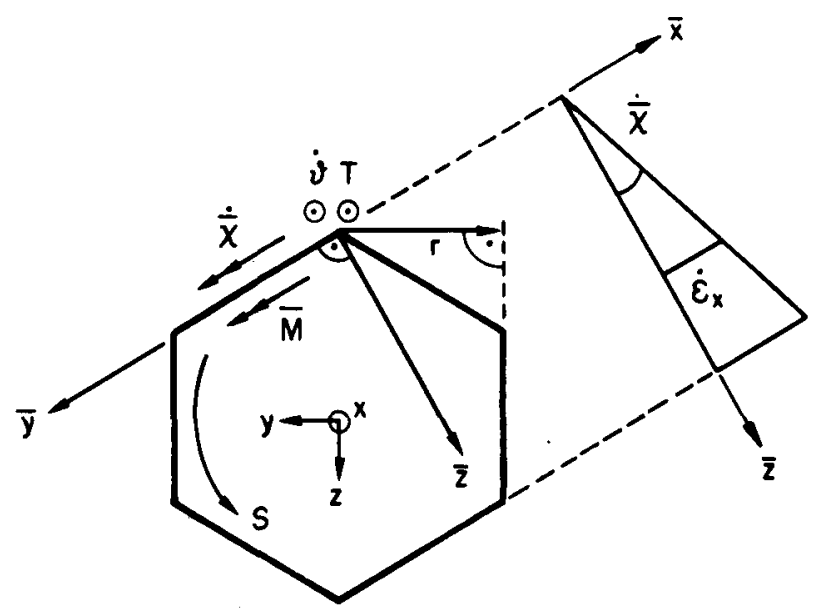

Fig. 8.7 Grundmechanismus für den polygonalen Querschnitt 
Euler'sche Differentialgleichung besonders einfach - nämlich finit - und lautet [8]

$\frac{\partial}{\partial \dot{\gamma}_{x s}}\left\{P_{s} \frac{\dot{\gamma}_{x s}^{2}}{4 \bar{z}} \frac{1}{\dot{x}}-\mu \dot{\gamma}_{x s}\right\}=0$,

$\dot{\gamma}_{x s}=\mu 2 \bar{z} \dot{\bar{X}} / P_{s}$,

wobei $\mu$ den konstanten Langrange-Multiplikator der Nebenbedingung bezeichnet. Einsetzen von (8.36) in die Nebenbedingung (8.35b) ergibt

$2 F_{0} \dot{\vartheta}=2 \mu \dot{\bar{x}} \oint \frac{\bar{z}}{P_{s}} d s$

$\mu=\frac{\dot{s}}{\dot{\bar{x}}} \frac{\mathrm{F}_{\mathrm{D}}}{\frac{\bar{z}}{\mathrm{P}_{\mathrm{s}}} \mathrm{ds}}$.

Mit (8.36) und (8.37) ist somit die Schiebungsgeschwindigkeit gegeben durch

$\dot{\gamma}_{x s}=\frac{2 F_{0}}{\oint \frac{\bar{z}_{P_{s}}}{P_{s}} \frac{\bar{z}}{P_{s}}} \dot{i}$.

Mit (8.38) und (8.27) kann nun auch die Wölbfunktion w bestimmt werden. Der Nullpunkt der Umfangskoordinate $s$ liegt im Ursprung des $(\bar{y}-\bar{z})$-Koordinatensystems.

$\omega(s)=\int_{0}^{s} \omega, s d s=\int_{0}^{s}\left(r-\dot{\gamma}_{X s} / \dot{\jmath}\right) d s$,

$w(s)=\int_{0}^{s}\left(r-\frac{2 F_{0}}{\oint \frac{\bar{z}}{P_{s}} d s} \frac{\bar{z}}{P_{s}}\right) d s$.

Die Schiebungsgeschwindigkeit und die Wölbfunktion, die für gegebene Werte von $\dot{\bar{\chi}}, \dot{\jmath}$ zur minimalen Dissipationsleistung führen, lassen sich offensichtlich durch Formeln beschreiben, die vollständig analog aufgebaut sind wie diejenigen, die sich unter den entsprechenden Verformungsannahmen in der elastischen Balkentheorie ergeben. Dabei entsprechen sich die Ausdrücke $\bar{z} / P_{S}$ und $1 / t$ : In der plastischen Balkentheorie ist aufgrund der Fliessbedingung (2.7 I) die Schiebungsgeschwindigkeit proportional zum Verlauf von $\dot{\varepsilon}_{x}$ und umgekehrt proportional zur Bügelfliesskraft $P_{s}$; in der elastischen Balkentheorie ist die Schiebung umgekehrt proportional zur Scheibendicke $t$.

Einsetzen von (8.38) in die Dissipationsleistung (8.34) des Querschnittes ergibt

$\left.\begin{array}{l}L_{d}^{Q}=H(\dot{\bar{X}}, \dot{v})=\dot{\bar{x}} \oint P_{x} \bar{z} d s+\frac{\dot{y}^{2}}{\frac{\dot{\bar{x}}}{\oint} \frac{F_{0}^{2}}{\frac{\bar{z}}{\mathrm{P}_{s}} d s},} \\ \dot{\bar{x}} \geq 0 .\end{array}\right\}$

Die Dissipationsleistung ist nun nur noch Funktion der verallgemeinerten Verformungen $\dot{\bar{x}}$, $\dot{\jmath}$ und kann aus der Sicht der Theorie der konvexen Körper [7] als Stützfunktion $H(\dot{\bar{X}}$, $\dot{\vartheta})$ eines konvexen Körpers im Raum der zu $\dot{\bar{\chi}}$, $\dot{v}$ dualen Grössen aufgefasst werden. Da die in Abschnitt 6.3 erläuterten Zuordnungsregeln für verallgemeinerte Spannungen und Verformungen jenen für 
duale Grössen entsprechen, ist mit $(8.40)$ die gesuchte Fliessfigur im $(\bar{M}, T)$-Raum in der Form ihrer stützfunktion gefunden. Für die Randpunkte der konvexen Fliessfigur gilt [2, 6 , 7 ]

$\bar{M}=\frac{\partial H}{\partial \dot{\bar{\chi}}}=\oint P_{x^{2}} \bar{z} d s-\frac{\dot{\vartheta}^{2}}{\dot{\bar{\chi}}^{2}} \cdot \frac{F_{0}^{2}}{\oint \frac{\bar{z}}{P_{s}} d s}$,

$T=\frac{\partial H}{\partial \dot{v}}=\frac{\dot{v}}{\dot{\bar{x}}} \frac{2 \mathrm{~F}_{\mathrm{O}}^{2}}{\oint \frac{\bar{z}}{\mathrm{P}_{\mathrm{s}}} \mathrm{ds}}$,

und durch Eliminieren von $\dot{\bar{x}}$, $\dot{\text { aus }}(8.41)$ und (8.42) erhält man die Interaktionsbeziehung für $\bar{M}$, T im Kollapszustand.

$$
\| \begin{aligned}
& \bar{M}=\oint P_{x} \bar{z} d s-\frac{T^{2}}{4 F_{0}^{2}}\left(\oint \frac{\bar{z}}{P_{s}} d s\right), \| \\
& \operatorname{mit} \bar{M}=f\left(N, M_{y}, M_{z}\right) .
\end{aligned}
$$

Die Formulierung von (8.43) für jede der $n$ Seiten eines Querschnittes ergibt $n$ Interaktionsbeziehungen $\operatorname{im}\left(N, M_{y}, M_{z}, T\right)$-Raum. Diese beschreiben die Fliessfigur in den verallgemeinerten Spannungen (8.29) vollständig, da alle mit dem Regime I verträglichen, unabhängigen Kambinationen der veraligemeinerten Verformungen (8.25) erfasst sind.

Zur Kontrolle kann man wieder die für positives Moment gültige Interaktionsbeziehung (8.4) für den Rechteckquerschnitt verifizieren, der in den Abschnitten 8.1 und 8.2 als Beispiel behandelt wurde (Fig. 8.8). Die Auswertung von (8.43) für in der obern Querschnittsseite liegende Neutralachse ergibt

$$
\begin{aligned}
\oint P_{x} \bar{z} d s & =2 Z_{f} h, \\
\oint \frac{\bar{z}}{P_{s}} d s & =2 \frac{h^{2}}{2} \frac{1}{P_{s}}+b h \frac{1}{P_{u}}, \\
& =F_{o}\left(\frac{h}{b} \frac{1}{P_{s}}+\frac{1}{P_{u}}\right), \\
\bar{M} & =2 Z_{f} h-\frac{T^{2}}{4 F_{0}}\left(\frac{h}{b} \frac{1}{P_{s}}+\frac{1}{P_{u}}\right) .
\end{aligned}
$$

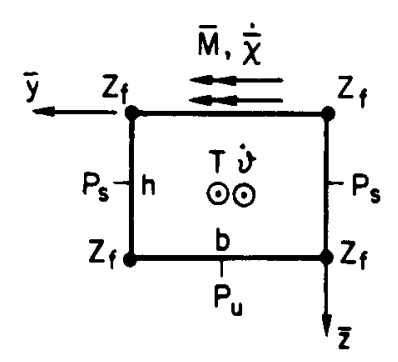

Fig. 8.8

Einsetzen von (8.42) in (8.37) erhellt die physikalische Bedeutung des Lagrange-Multiplikators $\mu$

$\mu=\frac{T}{2 F_{0}}$

Mit (6.5a), (8.36) und (8.30) folgt für die Neigung $\alpha$ der verschwindenden Hauptdehnungsgeschwindigkeit $\dot{\varepsilon}_{2}$

$\cot \alpha=\frac{\dot{\gamma}_{x s}}{2 \dot{\varepsilon}_{x}}=\frac{\mu}{P_{s}}=\frac{T}{2 F_{0}} \frac{1}{P_{s}}$,

wenn anders als in Abschnitt 6.1 der zu positivem $N_{x s}$ bzw. $\dot{\gamma}_{x s}$ führende Winkel $\alpha$ als positiv eingeführt wird. Die Betondrucktrajektorienneigung andererseits ergibt sich mit $N_{s}=0$ und 
$(6.3 a) \mathrm{zu}$

$\cot \alpha=\frac{N_{x s}}{P_{s}}=\frac{S}{P_{s}}$.

Der Querschnittsmechanismus, der für eine ebene Verteilung der Dehnungsgeschwindigkeit $\dot{\varepsilon}_{x}$ bei unbehinderter Verwölbung $\omega(s)$ zur minimalen Dissipationsleistung und Traglast führt, ist also - wie der Vergleich von (8.45) und (8.46) zeigt - verträglich mit Spannungszuständen, die einen konstanten Schubflussverlauf $S=T / 2 F$ aufweisen. Damit ist die Beziehung ( $8.29 b$ ) verifiziert, die die verallgemeinerte Spannung $T$ mit dem Schubfluss $S$ verknüpft.

In Kenntnis der vorliegenden Zusammenhänge kann man natürlich den Schritt von den verallgemeinerten Spannungen (8.31), (8.29b) zu den Interaktionsbeziehungen (8.43) wesentlich einfacher und schneller über die statische Seite vollziehen. In den Querschnittsseiten, die einen Beitrag zum Integral in (8.31) liefern, muss die Fliessbedingung (B.32) identisch erfüllt sein, und mit (8.29b) folgt

$N_{x}=P_{x}-\frac{T^{2}}{4 F_{0}} \frac{1}{P_{5}}$

Einsetzen von (8.47) in (8.31) ergibt unmittelbar (8.43). Dieses für die Praxis geeignetere Vorgehen entspricht genau den Gleichungen (8.1) bis (8.4) und dem in [18, 22] auch für allgemeine Querschnitte beschriebenen Verfahren.

Die hier bzw. in den vorangehenden Abschnitten beschriebenen Querschnittsmechanismen und räumlichen Kollapsrissmechanismen, die mit den Membrankräften beim Kollaps gemäss Fachwerkmodell verträglich sind, zeigen die theoretische Bedeutung dieses Vorgehens sowohl innerhalb der Balkentheorie als auch innerhalb der Plastizitätstheorie auf: Die aus dem vollständigen Satz von Interaktionsbeziehungen (8.43) gewonnene Fliessfigur im (N,M , M , T) -Raum liefert die plastizitätstheoretisch exakte Traglast aufgrund der Fliessbedingung (2.7 I) innerhalb einer Balkentheorie, die durch die Verformungsannahmen (8.24) bis (8.28) charakterisiert ist. Diese Verformungsannahmen entsprechen, wie insbesondere auch die Ausdrücke (8.38), (8.39) für die Schiebungsgeschwindigkeit und die wölbfunktion zeigen, genau jenen, die üblicherweise auch der elastischen Balkentheorie zugrunde gelegt werden. Damit fügen sich die Resultate des Fachwerkmodells nahtlos in die übliche Balkentheorie ein.

Im Vergleich zu der elastischen Balkentheorie besteht jedoch bei der plastischen Balkentheorie eine zusätzliche Schwierigkeit. Aus der elastischen Lösung des gemischten Torsionsproblems für konstantes Torsionsmoment ist bekannt, dass der Einfluss von Wölbbehinderungen am Balkenende rasch abklingt. Dies ist auch aufgrund des Prinzips von St. Venant zu erwarten. Ein solches existiert jedoch in der Plastizitätstheorie nicht.

Nun sind aber die im Querschnitt vorausgesetzten Dehnungsgeschwindigkeiten (B.26) nur dann mit dem Geschwindigkeitsfeld (8.24) kompatibel, wenn gemäss (8.25c) und (8.37) die Verdrehungs - und Krümmungsgeschwindigkeiten konstant sind. Innerhalb der Balkentheorie, die nur vollplastifizierte oder starre Querschnitte kennt, muss sich der Mechanismus über die gesamte Balkenlänge erstrecken, so dass sich alle Querschnitte inklusive der Endquerschnitte gleich verwölben. Damit würden aber kinematische kompatibilitäts- oder Randbedingungen an der Grenze zu starren Balkenzonen oder starren Lagerendblöcken verletzt. Letztere sind bei Stahlbetonbalken immer vorhanden zur Verankerung der Längsarmierung. Entsprechendes gilt in bezug auf die nicht verschwindende Bügeldehnung $\dot{\varepsilon}_{S}>0$ (siehe Abschnitt 6.3.3). 
Sie zeigen, dass die Mechanismuslänge in Wirklichkeit endlich sein kann. Die Verwölbung w(s) ist primär Ausdruck der Tatsache, dass nicht ein isolierter, plastifizierter Querschnitt fliessen kann, bzw. dass die Grenze zwischen starren und fliessenden Zonen nicht entlang eines zur Balkenaxe senkrechten Quersehnittes verlaufen kann.

Mit den Verzerrungsgeschwindigkeiten und verallgemeinerten Verformungen (8.30), (8.38) sind statt der Geschwindigkeitsfelder (8.24) eher diejenigen der Kollapsmechanismen gemäss

Fig. 8.6 zu assoziieren, deren Verformungen formal einem Querschnitt zugewiesen werden. Dies zeigt sich auch in den analogen Beziehungen zwischen Biege- und Torsionsrotation der Kollapsrissmechanismen einerseits und Krümmung und Verdrehung der Querschnittsmechanismen andererseits. Für deren Verhältnis folgt aus (8.37) und (8.45)

$\frac{\dot{v}}{\dot{X}}=\frac{1}{F_{0}} \oint \bar{z} \frac{\mu}{P_{s}} d s=\frac{1}{F_{0}} \oint \bar{z} \cot d s$.

Die Beziehung (8.48) ist offensichtlich identisch mit Gleichung (8.23) für die Neigung cot $\beta$ der Rotationsachse des Kollapsrissmechanismus. Eine unbehinderte Verwölbung darf also dann vorausgesetzt werden, wenn die Balkenlänge die Ausdehnung des massgebenden Kollapsrissmechanismus übertrifft.

\subsection{Ergänzungen}

\subsubsection{Zur Annahme nichtmassgebender Betonfestigkeit}

In den vorangehenden Abschnitten wurde immer vorausgesetzt, dass der betrachtete Balken unterarmiert sei: Armierungsgehalt und Betonabmessungen sollen so aufeinander abgestimmt sein, dass sich die plastifizierten Scheiben des dünnwandigen Balkens beim Kollaps im Fliessregime I befinden. Der Kollapsmechanismus muss dann der kinematischen Bindung $\dot{\varepsilon}_{1} \geq \dot{\varepsilon}_{2}=0$ genügen. Theoretisch wird damit jedoch die Membrankraft $n_{2}$ im Beton als interne Reaktion eingeführt, und es wird mit der ins Unendliche fortgesetzten Fliessfläche I gearbeitet. Es lässt sich deshalb nicht ausschliessen, dass die Gleichgewichtsbedingungen unter der Kollapslast gemäss Abschnitt 8.4 nur für eine lokal unendlich grosse Betonmembrankraft erfüllt sind.

Bei einem konvexen, polygonalen Querschnitt sind die Dehnungsgeschwindigkeiten (8.26) genau dann überall positiv, wenn sie in den $n$ Querschnittsecken grösser oder gleich null sind.

$\dot{\varepsilon}_{x i}=\dot{\varepsilon}+z_{i} \dot{x}_{y}+y_{i} \dot{x}_{z} \geq 0$,

für alle Querschnittsecken $i=1$ bis $n$.<smiles>C/C=C\CC</smiles>

Aus dualer, statischer sicht setzt man aber mit (8.49) voraus, dass in den Querschnittsecken beliebig grosse, konzentrierte Druckkräfte aufgenommen werden können. Die Traglasten gemäss Abschnitt 8.4 können deshalb dann und nur dann auch exakte Traglasten in bezug auf alle Fliessbedingungen (2.7) sein, wenn das Gleichgewicht im Kollapszustand ohne konzentrierte, vom Beton aufzunehmende Druckkräfte in den Querschnittsecken erfüllt ist. Ist diese notwendige (nicht hinreichende) Bedingung verletzt, kann es sich immer nur um obere Grenzwerte handeln.

Dass z.B. bei einem überwiegend auf schiefe Biegung beanspruchten, dünnwandigen Rechteckquerschnitt die Neutralachse des Mechanismus für endliche Betondruckfestigkeit normalerweise nicht mehr in einer Querschnittsseite liegen kann, sondern im Querschnittsinnern verlaufen muss, 
ist unmittelbar einsichtig. In [18] wird als Näherungslösung vorgeschlagen, die Interaktionsbeziehung zwischen (schiefem) Biegemoment und Torsionsmoment $M=f(T)$ mit dem Verhältnis der reinen Biegebruchmomente zu korrigieren, wie sie sich gemäss üblicher Biegetheorie einerseits $\left(M_{O B}\right)$ bzw. gemäss Abschnitt 8.4 andererseits $\left(M_{0}\right)$ ergeben: $M=f(T) \cdot M_{O B} / M_{0}$.

Die gleiche Situation kann jedoch auch bei reiner Torsionsbeanspruchung auftreten, wenn - wie das folgende Beispiel zeigt - die Armierung ungünstig eingelegt ist.

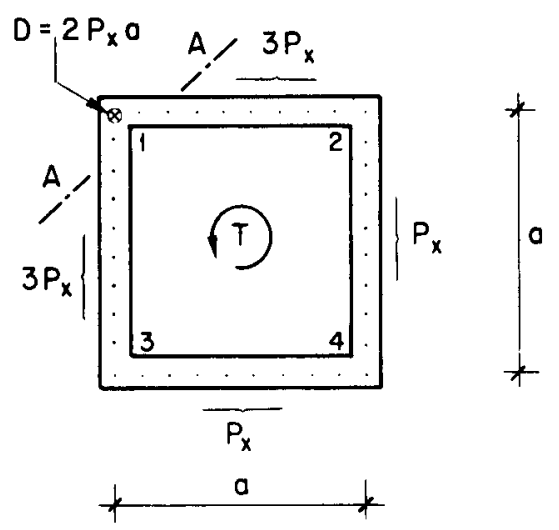

(a)

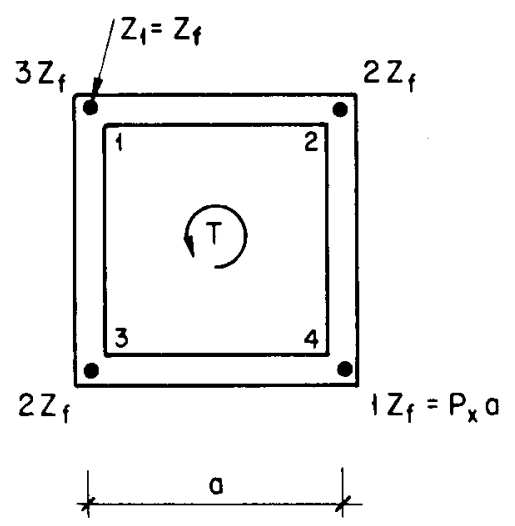

(b)

Fig 8.9 Einfluss der Armierungsanordnung auf die Betonspannungen ( $s$. Text)

Die beiden quadratischen, nur durch ein Torsionsmoment beanspruchten Querschnitte von Fig. 8.9 weisen in allen Querschnittsseiten die gleiche Bügelfliesskraft pro Längeneinheit $P_{S}$ auf. Die totale Fliesskraft der Längsarmierung ist ebenfalls für beide Querschnitte gleich. In Querschnitt (a) ist die Längsarmierung fein über die Querschnittsseiten verteilt (Fliesskraft pro Längeneinheit $P_{x}$ bzw. 3P ${ }_{x}$ ), während sie in Querschnitt (b) statisch äquivalent in den Querschnittsecken konzentriert ist.

Die Auswertung von (8.43) ergibt für beide Querschnitte das gleiche Torsionsbruchmoment und die gleichen massgebenden Kollapsmechanismen, nämlich die Mechanismen mit Neutralachse in den Seiten 1-2 oder 1-3. Da diese beiden Mechanismen auch kombiniert auftreten können, muss beim Kollaps die gesamte Armierung auf Zug fliessen mit Ausnahme der unmittelbar in der Ecke 1 situierten Längsarmierung. Man verifiziert leicht, dass im Fall (a) die Gleichgewichtsbedingungen im Kollapszustand nur erfüllt sind, wenn in der Querschnittsecke 1 eine konzentrierte Druckkraft der Grösse $D=2 P_{x}$ a übertragen werden kann. Für endliche Betondruckfestigkeit $P_{b}$ muss die neutrale Axe des Kollapsmechanismus im Querschnittsinnern liegen (A-A, Fig. 8.9 a), und der mit (8.43) berechnete Wert für das Torsionsbruchmoment kann nur oberer Grenzwert sein. Im Falle (b) dagegen ist die Längsarmierung in der Ecke 1 im Kollapszustand immer noch auf Zug beansprucht, $z_{1}=z_{f} \leq 3 z_{f}$, und das Torsionsbruchmoment gemäss (8.43) kann auch bei endicher Betondruckfestigkeit die exakte Traglast sein.

Das Beispiel zeigt sehr anschaulich, dass bei torsionsbeanspruchten Trägern die Konzentration der Längsarmierung in den Querschnittsecken die Beanspruchung des Betans und die Traglast günstig beeinflusst. Bei wirklichen Trägern mit endlichen Bügelabständen ist eine starke Eckarmierung zudem auch aus konstruktiver Sicht erforderlich, um ein Ausbrechen der Druckdiagonalen zwischen den Bügeln zu verhindern. 
Schliesslich ist auch zu erwähnen, dass die Betonspannungen beim Kollaps die Betondruckfestigkeit auch dann erreichen, wenn aufgrund der Membrantheorie der Abschnitte 8.2 bis 8.4 überall $n_{2}<P_{b}$ gilt. Der in Abschnitt 8.5.4 behandelte Mechanismus zeigt anschaulich, dass die Membrankraft $n_{2}$ beim Kollaps - gleich wie die Druckresultierende im Druckflansch bei reiner Biegung - über die kleinstmögliche an der Balkenoberfläche liegende Betondruckzone übertragen wird.

\subsubsection{Wölbtorsion und gemischte Torsion}

In verschiedenen Torsionsversuchsreihen weisen die Versuchsträger einen relativ kurzen Testbereich auf, während der restliche Balken - um den Bruch- und Messbereich eindeutig festzulegen - verstärkt ist. Es fragt sich, wie sich eine Behinderung der Längsausdehnung des Mechanismus von Fig. 8.4 auswirkt. Im Zusammenhang mit dem Einbezug des Querkrafteinflusses stellt sich weiter die Frage, wie die Querkraft auf die verschiedenen Scheiben aufzuteilen ist. Beide Fragen hängen eng zusammen. Sie können sehr anschaulich an folgendem einfachem Beispiel behandelt werden; das Resultat gilt jedoch auch für allgemeinere Armierung und Beanspruchung.

Der in Fig. 8.10 a dargestellte dünnwendige Kastenträger sei lediglich durch ein Torsionsmoment beansprucht und weise in allen vier Scheiben über die gesamte Länge die gleiche Bügelfliesskraft pro Längeneinheit $P_{s}$ auf. Die Längsarmierung bestehe nur aus vier Eckstringern mit der gleichen Fliesskraft $Z_{f}$ im Testbereich der Länge L. Ausserhalb des Testbereichs sollen die Eckstringer eine so grosse Fliesskraft aufweisen, dass sie als starr betrachtet werden dürfen.

Beim Kollaps erreicht aus Symmetriegründen die gesamte Armierung die Fliessgrenze, und als damit verträgliche Kollapsmechanismen kommen sowohl jeder Grundmechanismus (Fig. B.4) als auch ihre Kombinationen (Fig. 8.5) in Frage.

Da ausserhalb des Testbereichs nur die Längsarmierung verstärkt ist, kann sich der in Fig. 8.10 a dargestellte Mechanismus dann unbehindert ausbilden, wenn die Teststrecke L grösser ist als der Abstand der Punkte $B$ und $E$, wo die beiden unteren Stringer von den geöffneten Kollapsrissen gekreuzt werden und fliessen müssen. Dieser Abstand wird deshalb als massgebende Mechanismuslänge 1 bezeichnet und beträgt

$1=b \cot \beta-2 h \cot \alpha_{s}$.

Die Endquerschnitte bleiben eben und ihre Form bleibt erhalten, wenn der Mechanismus Gleichung (8.12) bzw. mit $\alpha_{v}=\alpha_{h}=\alpha_{s}$ der Beziehung

$b \cot \beta=h \cot \alpha_{s}+b \cot \alpha_{u}$,

genügt. Einsetzen von $(8.51)$ in $(8.50)$ ergibt

$1=b \cot \alpha_{u}-h \cot \alpha_{s}$.

Nimmt man an, dass sich die Mechanismuslänge frei einstellen kann, so ist gem. Absch. 8.2 bis 8.4 die exakte Lösung des Traglastproblems bekannt. Der Kollapsspannungszustand weist einen über den Querschnittsumfang konstanten Schubfluss $S_{0}$ auf, und die zugehörige freie Mechanismuslänge $I_{F}$ lässt sich mit $\cot \alpha_{S}=\cot \alpha_{u}=S_{O} / P S_{S}$ ausdrücken durch 
(a)
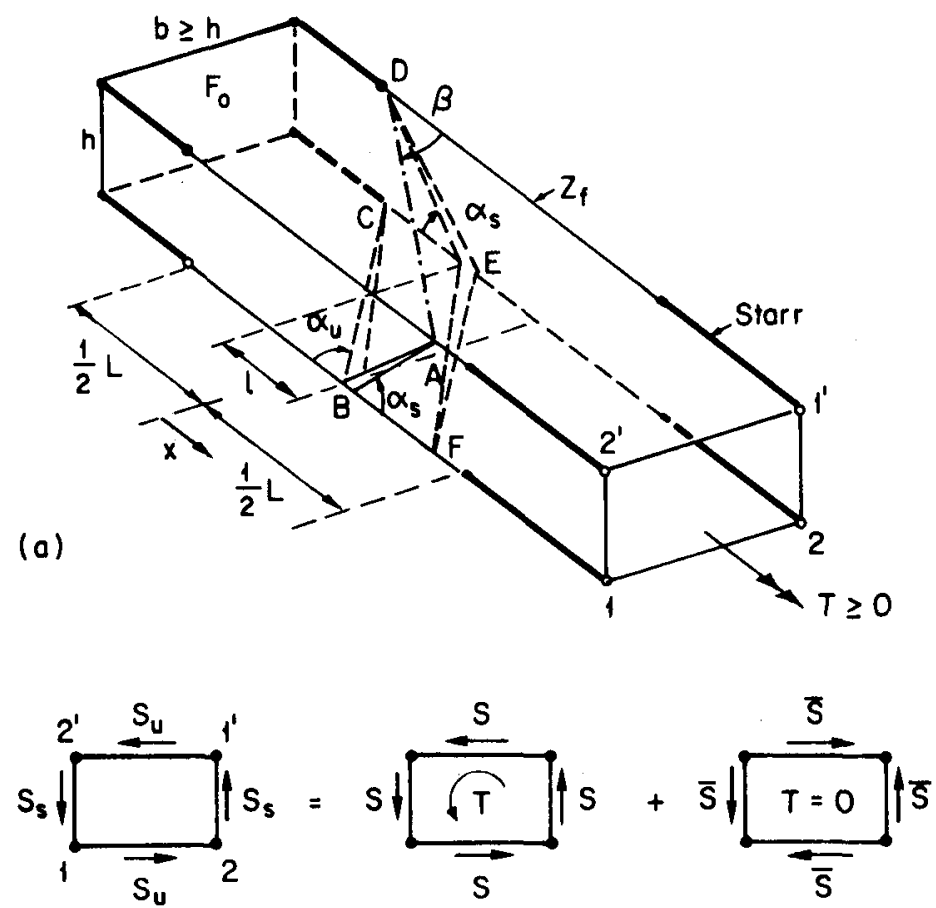

(b)

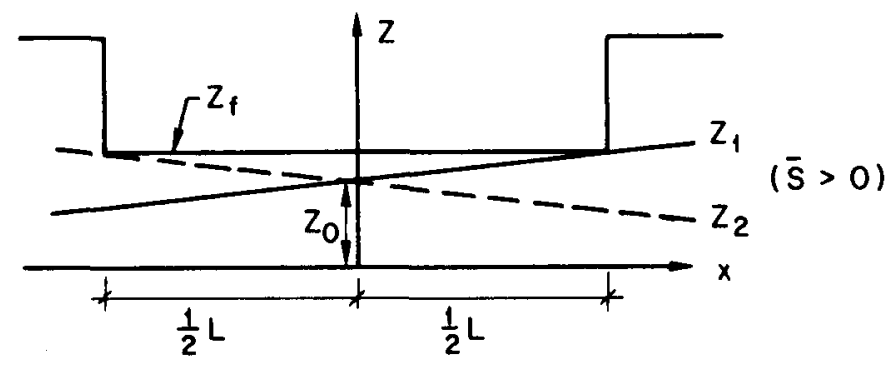

(c)

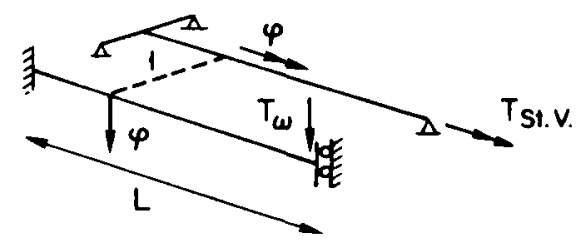

Fig. 8.10 Beschrönkte Mechanismuslönge

$\left.\begin{array}{l}1_{F}=\frac{S_{D}}{P_{S}}(b-h), \\ S_{0} \geq 0, \quad b \geq h .\end{array}\right\}$

Die exakte Traglast ist also mit $T=2 F_{0} S_{0}$ bekannt, solange die Teststrecke grösser als die freie Mechanismuslänge ist, $L \geq l_{F}$. Ist jedoch $L<l_{F}$, so muss der Mechanismus der zusätzlichen kinematischen Restriktion $l \leq L<{ }_{F}$ genügen, und die Mechanismusmethode liefert nun höhere Traglasten $T / 2 F_{0}=S>S_{0}$. Es ist somit zu überprüfen, ob für $L<I_{F}$ statisch zulässige Spannungszustände mit nichtkonstantem Schubfluss unter höheren Torsionsmomenten stabil sind als solche mit konstantem Schubfluss.

Der im folgenden untersuchte Spannungszustand (Fig. 8.10 b) weist in den einzelnen Scheiben des. Trägers immer noch konstante Beton- und Bügelspannungen auf, und der Schubflussverlauf 
ist somit entlang den einzelnen Querschnittseiten immer noch konstant. Die Schubflüsse $S_{S}$ und $S_{u}$ in den seitlichen bzw. unteren/oberen Querschnittseiten brauchen aber nicht gleich gross zu sein, und entsprechend der Schubflussdifferenz $S_{s}{ }^{-} S_{u}$ variiert auch der Zugkraftverlauf in den Eckstringern. Der Schubfluss wird mit Vorteil in einen über den Querschnittsumfang konstanten Anteil $S$ und einen Eigenspannungszustand $\bar{S}$ aufgeteilt (Fig. 8.10 b)

$\left.\begin{array}{l}s_{s}=s+\bar{s}, \\ s_{u}=s-\bar{s}, \\ s=T /\left(2 F_{0}\right) .\end{array}\right\}$

Aus Symmetriegründen müssen die vier Eckstringer im Querschnitt $x=0$ die gleiche Zugkraft $Z_{0}$ aufweisen. Aus dem Längsgleichgewicht und der identisch erfüllten Fliessbedingung (8.32), $\left(P_{x}=0\right)$, folgt sofort

$z_{0}=-\frac{1}{4} \oint N_{x} d s=\frac{1}{2 P_{s}}\left(h s_{s}^{2}+b S_{U}^{2}\right)$

In den kritischen Querschnitten $x= \pm$ L/2 beträgt somit die grösste Eckstringerkraft

$z_{\text {max }}(x= \pm L / 2)=z_{0}+\frac{L}{2}\left|s_{s}-s_{u}\right|$

Der Spannungszustand ist stabil für $z_{\max }=z_{f}$, und mit (8.54), (8.55), (8.56) ergibt sich folgende, implizite, noch von $\bar{S}$ abhängige Bestimmungsgleichung für $S=T /\left(2 F_{0}\right)$

$Z_{f}=\frac{1}{2 P}\left(h S_{s}^{2}+b S_{u}^{2}\right)+L|\bar{S}|$

Es ist bekannt, dass die Funktion $S(\bar{S})$ für $L \geq{ }_{F}$ ihr Maximum $S_{0}$ an der Stelle $\bar{S}=0$ annimmt. Es interessiert deshalb nur noch, ob sie im Falle $L<I_{F}$ für $\bar{S} \neq 0$ maximal wird. An einem Maximum in $\bar{S} \neq 0$ muss aber $\partial S / \partial \bar{S}=0$ gelten und somit auch

$\frac{\partial S_{S}}{\partial \bar{S}}=\frac{\partial S}{\partial \bar{S}}+1=1, \frac{\partial S_{U}}{\partial \bar{S}}=-1$.

Ableiten von (8.57) nach $\bar{S}$ und Berücksichtigung von (8.58) ergibt die Maximumsbedingung

$L=\frac{1}{P_{S}}\left(b S_{U}-h S_{S}\right)$

wobei $\overline{\mathrm{S}} \geq 0$ vorausgesetzt ist. Durch Einsetzen von (8.54) in (8.59) und Auflösen nach $\overline{\mathrm{S}}$ erhält man

$\bar{S}=\frac{P_{S}}{b+h}\left(\frac{S}{P_{S}}(b-h)-L\right)$.

Da mit $S=S_{0}$ ein unterer Grenzwert bekannt ist, gilt sicher $S \geq S_{0}$, und mit (B.53) folgt aus $(8.60)$

$\bar{S} \geq \frac{P}{b+h}\left(I_{F}-L\right)$.

Aus (8.61) ist nun aber sofort ersichtlich, dass sich das grösstmögliche Torsionsmoment im Falle $l_{F}>L$ für einen nichtkonstanten Schubfluss, $\bar{S}>0$, ergibt. 
Es fragt sich nun noch, ob dieser Spannungszustand die exakte Traglast liefert. Drückt man in der Maximumsbedingung (8.59) die Schubflüsse durch die Neigungen der Betondrucktrajektorien aus,

$L=b \cot \alpha_{u}-h \cot \alpha_{s}$,

so zeigt der Vergleich von (8.62) mit (8.52), dass die massgebende Länge des mit dem Betonspannungszustand verträglichen Mechanismus gerade gleich der Teststrecke L ist. Die Punkte $B$ und $E$ der beiden Kollapsrisse liegen also in den Querschnitten $x=+L / 2$ bzw. $x=-L / 2$ (Fig. 8.10 a), wo die Stringer 1 bzw. 2 für $\vec{S}>0$ an der Fliessgrenze liegen (Fig. 8.10 b). Der Mechanismus ist also auch mit den Stringerspannungen verträglich, und somit ist auch für $L<I_{F}$ die exakte Lösung aufgrund der Fliessbedinung $(2.7$ I) gefunden.

Die variablen Zugkraftanteile in den vier Eckstringern stellen natürlich ein nichtkonstantes Wölbmoment dar, und entsprechend kann man das Torsionsmoment als Summe eines St. Venant'schen Torsionsmomentes und eines Wölbtorsionsmomentes auffassen. Da die Endquerschnitte eben und die Stringer ausserhalb des Testbereichs starr vorausgesetzt wurden, weisen die Querschnittsecken in $x= \pm L / 2$ eine wälbeinspannung auf. Das entsprechende statische system des gemischten Torsionsproblems ist - unter Verwendung der Analogie zur Biegetheorie - in Fig. $8.10 \mathrm{C}$ skizziert.

Die freie Mechanismuslänge $l_{F}$ hat also eine klare physikalische Bedeutung: Sie ist ein Mass dafür, ob ein rein St. Venant'sches Torsionsproblem ( $L \geq l_{F}$ ) oder ein gemischtes Torsionsproblem $\left(L \leq I_{F}\right.$ ) vorliegt. Insbesonderè verifiziert man auch leicht, dass sich die Tragwirkung des Balkens bei abnehmender Bügelarmierung in der untern und obern Schubwand mit

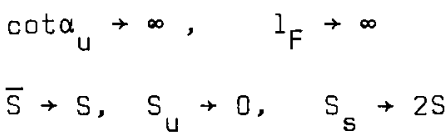

dem Fall reiner Wälbtorsion nähert. Das Beispiel verdeutlicht damit noch einmal den am Ende der Abschnitte 8.2 und 8.4 erwähnten Zusammenhang zwischen unbehinderter Längsausdehnung des Kollapsrissmechanismus und der freien Verwölbung des vollplastifizierten Querschnittes bei der reinen Querschnittsbetrachtung gemäss Abschnitt 8.4.

Dem Beispiel kann noch ein weiteres, im folgenden benötigtes Resultat entnommen werden. Betrachtet man in Fig. 8.10 nur einen isolierten Querschnitt $(z \cdot B . \times=0)$ und fragt nach dem optimalen Eigenspannungszustand $\overline{\mathrm{S}}$, der in diesem isolierten Querschnitt zum grössten Torsionsmoment führt, so ist die in den Schubflüssen bzw. Drucktrajektorienneigungen ausgedrückte Maximumsbedingung offensichtlich wieder durch (8.59), (8.60), (8.62) gegeben, wenn dort $L=0$ gesetzt wird. Der Vergleich der nun durch

$b \cot \alpha_{u}-h \cot \alpha_{S}=0$

gegebenen Gleichung (8.62) mit (8.52) zeigt, dass für den mit den Trajektorienneigungen (8.63) verträglichen Mechanismus der Abstand 1 der Punkte $B$ und E verschwindet. Die beiden Kollapsrisse kreuzen also die Eckstringer im betrachteten Querschnitt und sind damit auch mit den Stahlspannungen in den Stringern verträglich, die in diesem Querschnitt an der Fliessgrenze liegend vorausgesetzt wurden: Superponiert man also beim Vorgehen nach dem statischen Grenzwertsatz zu einer statisch zulässigen Schubflussverteilung den für den betrachteten Querschnitt optimalen Schubfluss $\bar{S}$, so entspricht dies in der dualen Problemformulierung aufgrund des kinematischen Grenzwertsatzes der Annahme, dass die Querschnittsverwölbung in 
den Querschnittsecken verschwinden muss $(L=0:)$. Diese Wölbbehinderung drückt sich im räumlichen Kollapsmechanismus darin aus, dass die beiden Kollapsrisse die Eckstringer im betrachteten Querschnitt kreuzen müssen $(1=0$ :). Diese Feststellung gilt auch, wenn die Querschnittsseiten verschieden starke Bügelarmierung aufweisen oder zusätzlich durch Schub infolge Querkraft beansprucht sind.

\subsubsection{Zum Einbezug der Querkraft}

Die Ableitung von Querschnittsinteraktionsbeziehungen für unterarmierte, rechteckige Kastenträger unter Biegung, Torsion und Querkraft erfolgt meist unter den folgenden Annahmen [22, 23] :

1. Das Torsionsmoment wird durch einen über den Querschnittsumfang konstanten Schubfluss aufgenommen.

2. Die mittig zwischen den zwei Stegen angreifende Querkraft wird durch zwei gleich grosse, konstante Schubflüsse in den Stegen aufgenommen.

3. Die Tragfähigkeit des Querschnittes ist erschöpft, wenn die Bügel und die Längsarmierung im betrachteten Querschnitt in mindestens drei Querschnittsseiten fliessen. Besteht die Längsarmierung aus vier Eckstringern, so müssen also im betrachteten Querschnitt zwei benachbarte Eckstringer fliessen.

Nun können die Kollapsmechanismen bei Einbezug der Querkraft kaum grundsätzlich anders aussehen als bei reiner Biegung und Torsion. Andererseits ist die engenommene, statisch zulässige Schubflussverteilung im allgemeinen nicht die optimale im Sinne der obigen Ueberlegungen. Der Abstand 1 der Punkte $B$ und $E$ (Fig. 8.10 a) desjenigen Kollapsmechanismus, der mit den aus den Annahmen 1 und 2 resultierenden Trajektorienneigungen verträglich ist, wird deshalb im allgemeinen nicht null sein. Da somit nicht beide Kollapsrisse die Eckstringer im Betrachteten Querschnitt kreuzen können, kann der Mechanismus auch nicht mit dem in diesem Querschnitt angenommenen Spannungszustand verträglich sein.

Gewinnt man andererseits eine M-Q-T-Interaktionsbeziehung, indem der angenommenen, statisch zulässigen Schubflussverteilung noch ein optimaler Schubfluss $\vec{S}$ superponiert wird, so gibt es einen Grundmechanismus gemäss Fig. 8.10 a, dessen Kollapsrisse die Eckstringer im betrachteten Querschnitt kreuzen, und der auch mit den resultierenden Neigungen der Drucktrajektorien verträglich ist. Man überschätzt dann aber den Bruchwiderstand des Querschnittes sicher zumindest im Falle reiner Torsion und Biegung.

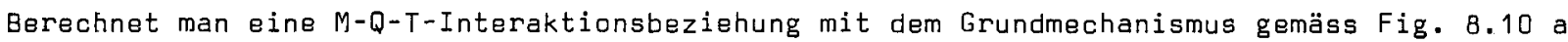
unter der Annahme, dass die beiden Punkte $B$ und $E$ nicht im betrachteten Querschnitt, sondern nur symmetrisch zu diesem liegen, so ergeben sich die gleichen Neigungen für die Kollapsrisse bzw. Betondrucktrajektorien und die gleiche Interaktionsbeziehung wie beim statischen Vorgehen gemäss Annahmen 1 bis 3. Der mit diesem Mechanismus verträgliche Spannungszustand, der im betrachteten Querschnitt einen seitenweise konstanten Schubfluss aufweist, ist dort aber in einem Eckstringer nicht stabil.

Schliesslich lässt sich zeigen, dass die Spannungsansätze gemäss Annahmen 1 bis 3 und der letztgenannte Mechanismus der Kombination der Balkentheorie von Abschnitt 6.3 mit derjenigen von Abschnitt 8.4 entsprechen. Aus den vorstehenden Ueberlegungen muss geschlossen werden, dass bei nicht verschwindender Querkraft der Querschnitt als wölbbehindert zu betrachten ist, und dass sich räumliche Kollapsrissmechanismen nur mit Querschnittsinteraktionsbeziehungen 
assoziieren lassen, die auch das wölbmoment und das Wölbtorsionsmoment als verallgemeinerte Spannungen enthalten.

\subsubsection{Mechanismen für Balken mit Vollquerschnitt}

In den Abschnitten 8.1 bis 8.4 sind bisher nur Balken unter konstantem Biege- und Torsionsmoment betrachtet worden, die einen dünnwandigen, geschlossenen Querschnitt aufweisen. Die Versuchsergebnisse zeigen aber, dass sich die erreichten Bruchlasten von Balken mit Hohlund Vollquerschnitten bei gleichen Aussenabmessungen und gleicher Armierung kaum unterscheiden, wenn der Kollaps durch Fliessen der Längs-und Bügelarmierung ausgelöst wird. Ist für den Balken mit Hohlquerschnitt ein stabiler, statisch zulässiger Spannungszustand bekannt, so gilt dieser natürlich auch für den Balken mit Vollquerschnitt, wenn der Kernbeton spannungsfrei angenommen wird. Die für den Hohlquerschnitt ermittelte exakte Traglast darf deshalb sicher als unterer Grenzwert für den Vollquerschnitt betrachtet werden. Damit sie auch für den Vollquerschnitt als oberer Grenzwert und damit exakte Traglast betrachtet werden darf, ist nachzuweisen, dass sich die Kollapsrisse der Mechanismen für den Hohlquerschnitt in kinematisch zulässiger und mit den im Balkenkern verschwindenden Betonspannungen verträglicher Weise ins Balkeninnere fortsetzen lassen. Für Balken mit rechteck- und trapezförmigem Vollquerschnitt, bei welchem die Mechanismen noch relativ leicht überblickbar sind, kann nun dieser Nachweis tatsächlich sofort erbracht werden.

Es wird wieder der Balken in Fig. 8.10 a $\left(L>I_{F}\right)$ betrachtet, der nun jedoch einen Vollquerschnitt aufweisen soll. Breite und Höhe bezeichnen nun die Aussenabmessungen des Querschnittes beim Kollaps. Diese entsprechen normalerweise den Aussenabmessungen des Armierungskorbes, da sich die Betonüberdeckung beim Kollaps meist schon gelöst hat. Ausgangspunkt für die nachfolgenden Ueberlegungen ist die aus (8.12) bzw. (8.23) folgende Tatsache, dass die Endpunkte A,F,C,D der beiden Kollapsrisse (Fig. 8.10 a) immer in einer Ebene liegen. Sind die Kollapsrissneigungen in der vorderen und hinteren Balkenseite gleich gross, $\alpha_{v}=\alpha_{h}=\alpha_{s}$, so steht die Ebene gemäss Fig. 8.10 a senkrecht auf der unteren Balkenseite; andernfalls liegt sie schief. Die Ebene enthält insbesondere auch die beiden Verbindungsgeraden A-C, D-F der Kollapsrissenden und die Rotationsachse A-D. Damit liegt die Vermutung nahe, dass sich beim Kollaps des Balkens mit Vollquerschnitt im Balkeninnern Kollapsrisse entlang den drei Ebenen $A B C$, DEF und AFCD öffnen: Während die vordere Balkenhälfte starr um die Rotationsachse A-D rotiert, öffnet sich entlang der Ebene AFCD ein abgeschlossener Hohlraum, indem die beiden Ufer der Rissebene um die Rotationsachsen A-C bzw. D-F ausknicken. Dabei rotieren die unteren Ufer der Rissebenen ABC bzw. DEF ebenfalls um die Rotationsachsen A-C bzw. D-F, und damit öffnen sich die auch auf der Balkenoberfläche sichtbaren Kollapsrisse A-B-C bzw. D-E-F.

Der Mechanismus lässt sich sehr anschaulich darstellen, wenn vorerst angenommen wird, dass sich bei der Rotation der vorderen Balkenhälfte um A-D ein schiefer Biegeriss entlang der Ebene ADCF öffnet (Fig. 8.10 a). Das hintere Rissufer befindet sich dann in der Position ADCF' (Fig. 8.11 a), das vordere in der Position ADC'F (Fig. 8.11 b). Durch den fiktiven Biegeriss und die Rissebenen $A B C$ bzw. DEF werden von der vorderen und hinteren Balkenhälfte die Tetraeder ABFC bzw. CDEF losgetrennt (Fig. 8.10 a). Rotiert nun der hintere Tetraeder um die Achse A-C in die Position ACF (Fig. 8.11 a) und der vordere um die Achse D-F in die Position DFC (Fig. 8.11 b), so öffnen sich einerseits die ebenen Kollapsrisse ABC bzw. DEF. Andererseits ist nun der fiktive Biegeriss auf der Balkenoberfläche wieder geschlossen, da sich - wie Fig. 8.11 zeigt - die beiden Rissflächen entlang ihres verformten Randes ADCF nahtlos zusammenfügen lassen. Uebrig bleibt ein abgeschlossener Hohlraum im Balkenkern, was sofort aus dem in Fig. 8.11 c dargestellten Längsschnitt entlang der Balkenmittellinie ersichtlich ist. 

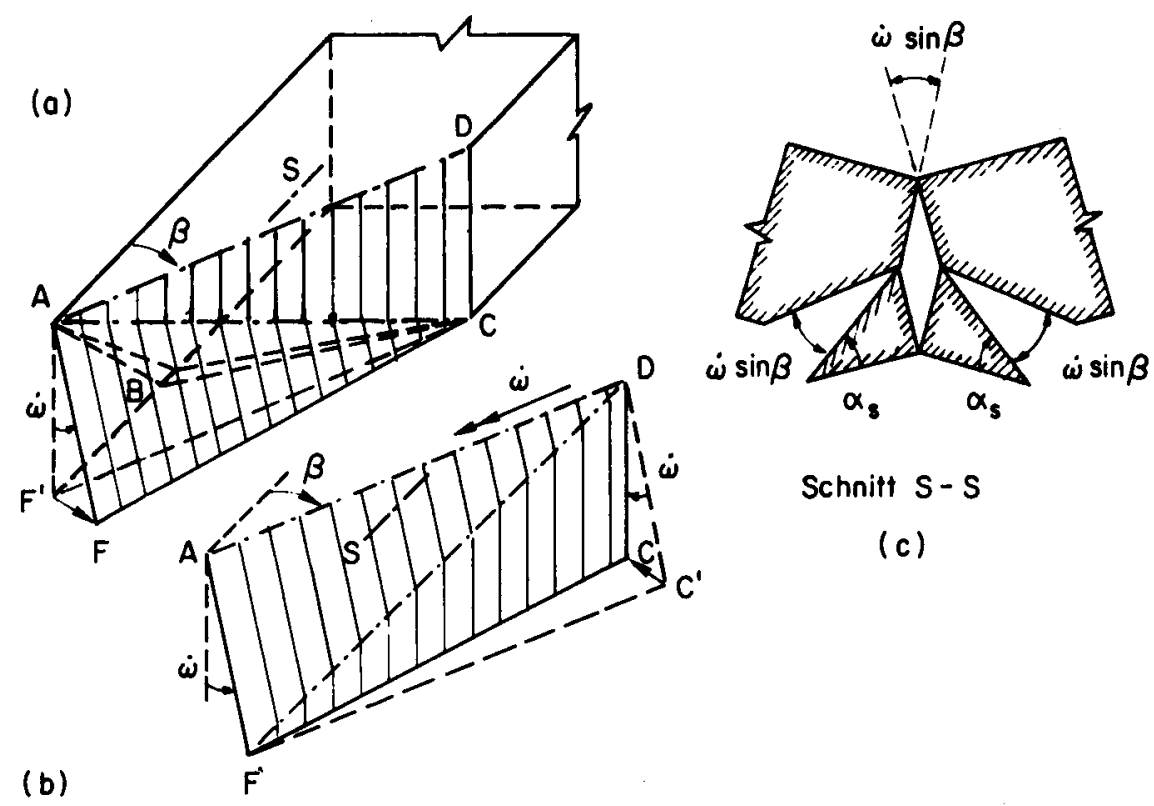

Schnitt S-S

(c)

Fig. 8.11 Grundmechanismus für Balken mit rechteckigem Vollquerschnitt

Der Mechanismus ist offensichtlich kinematisch zulässig, solange die Kollapsrissneigungen $\alpha$ und die Neigung $\beta$ der Rotationsachsen durch die Beziehungen (8.12) bzw. (8.23) verknüpft sind. Da die Geschwindigkeitsvektoren überall senkrecht auf den angenommenen Rissflächen stehen und sich die Risse überall öffnen, ist die Dissipationsleistung des Betons null. Der Mechanismus liefert somit für den Vollquerschnitt die gleichen oberen Grenzwerte wie für den Hohlquerschnitt. Insbesondere ist damit auch nachgewiesen, dass die zur Scheibenebene senkrechten Komponenten der Geschwindigkeitssprünge in Fig. 8.4 sowohl kinematisch zulässig als auch mit dem Spannungszustand im Beton verträglich sind.

Man überlegt sich leicht, dass die unteren Grenzwerte für die Traglast, die sich aus ebenen, stabilen, statisch zulässigen Spannungszuständen entlang des Querschnittumfangs errechnen (Fachwerkmodel1), dann mit den oberen Grenzwerten zusammenfallen, wenn die für die Stabilität der Betonspannungen erforderliche mitwirkende Wandstärke vernachlässigbar ist gegenüber den Querschnittsabmessungen. Der Mechanismus zeigt aber auch, dass nur innerhalb der Membrantheorie der Abschnitte 8.2 bis 8.4 die exakte Traglast gefunden ist. In einer kontinuumsmechanisch exakten Lösung, die euch die Verteilung der Spannungen und Verzerrungsgeschwindigkeiten über die Wanddicke einbezieht, muss die Kontaktfläche entlang des umlaufenden Knicks ADCF (Fig. 8.11) endlich sein, und es müssen auch hier Verträglichkeitsbedingungen erfüllt werden. Die Herleitung einer kontinuumsmechanisch exakten Lösung überschreitet den Rahmen dieser Arbeit und bleibt einer späteren Veröffentlichung vorbehalten. Sie würde im wesentlichen aber nur die von der Betondruckfestigkeit abhängige Ausdehnung der Betondruckzone und damit den Hebelarm der inneren Kräfte bzw. die vom Schubfluss umflossene Fläche Fo liefern, die innerhalb der Membrantheorie iterativ bestimmt oder geschätzt werden müssen.

Das experimentelle Ergebnis, wonach der Kernbeton keinen Beitrag zum Bruchwiderstand torsionsbeanspruchter Stahlbetonbalken leistet und nur eine dünne äussere Betonschale mitwirkt, kann also von der Plastizitätstheorie ebenfalls erfasst werden. Mit diesem Resultat, das wieder auf die Bedeutung hinweist, die dem plastizitätstheoretischen Ansatz zur Erfassung des Bruchwiderstandes schubbeanspruchter Stahlbetonbalken zukommt, soll der vorliegende Abschnitt abgeschlossen werden. 
K A P I TEL III

SCHEIBENTHEORIE

Die Balkentheorie der vorangehenden Abschnitte fusst auf der Voraussetzung, dass sich der Spannungszustand in Balkenlängsrichtung über Strecken in der Grössenordnung der Querschnittsabmessungen (Mechanismuslänge:) vernachlässigbar ändert. Nun liegen aber bei nicht verschwindender Querkraft die massgebenden Querschnitte wegen des Momentengradienten vielfach im Bereich von Einzellasten. Auflagern. Verstärkungen oder Sprungstellen des Armierungsgehaltes, wo die Betonspannungsfelder im Steg gerade stark variieren. Das Problem der Schubtragfähigkeit von Stahlbetonbalken mit dünnen Stegen ist deshalb vielfach ein Scheibenproblem. Insbesondere ist die sogenannte Sprengwerk- oder Bogenwirkung ein typisches Scheibenproblem. Andererseits lassen die Ergebnisse der Abschnitte 6 bis 8 vermuten, dass die Plastizitätstheorie auch für wandartige Träger eine vernünftige Abschätzung der Tragfähigkeit zu liefern vermag.

In den folgenden drei Abschnitten werden zuerst die allgemeinen Spannungs- und Geschwindigkeitsfelder der plastifizierten Stahlbetonscheibe diskutiert. Anschliessend werden die erhaltenen Beziehungen auf spezielle Schubprobleme bei Stahlbetonbalken und auf einen wandartigen Träger angewandt.

\section{PLASTIZITAETSTHEORIE DER STAHLBETONSCHEIBE}

\subsection{Spannungsfelder im Regime I}

In einem im Regime I vollplastifizierten Gebiet genügen die Membranspannungen dem Gleichungssystem

$$
\left.\begin{array}{ll}
N_{x, x}+N_{x y, y} & =0, \\
N_{x y, x}+N_{y, y} & =0, \\
\left(P_{x}-N_{x}\right)\left(P_{y}-N_{y}\right) & =N_{x y}^{2},
\end{array}\right\}
$$

wenn das Eigengewicht der Scheibe vernachlässigt wird. Ist - was meist zutrifft - $P_{x}$ in $x$ und $P_{y}$ in $y$-Richtung konstant, so leistet die Armierung wegen $z_{x}=P_{x}, z_{y}=P_{y}$ keinen Beitrag zu (9.1). Es brauchen nur die Betonspannungen betrachtet zu werden und die Stahlspannungen treten lediglich in den Randbedingungen auf. Mit (2.1) schreiben sich (9.1), (9.2)

$$
\left.\begin{array}{l}
n_{x, x}+n_{x y, y}=0, \\
n_{x y, x}+n_{y, y}=0, \\
n_{x} n_{y}=n_{x y}^{2}, \text { bzw. } n_{1}=0 .
\end{array}\right\}
$$


Erfüllen der Gleichgewichtsbedingungen (9.3) mit Hilfe der Spannungsfunktion $F$,

$\left.\begin{array}{l}n_{x}=-F_{, y y}, \\ n_{y}=-F_{, x x}, \\ n_{x y}=F_{, x y},\end{array}\right\}$

und Einsetzen von $(9.5)$ in $(9.4)$ ergibt

$F_{x y}^{2}=F, x x, F$,

Gleichung (9.6) ist die Differentialgleichung aller abwickelbaren Flächen, d.h. aller Enveloppen, einer einparametrigen Ebenenschar. Diese können,mit Ausnahme der Ausartungen Ebenen, Zylinder- und Kegelflächen, auch als diejenigen Flächen definiert werden, die von den Tangenten einer Raumkurve aufgespant werden. Deren Projektion in die $x-y$-Ebene wird durch eine einparametrige Geradenschar und ihre Hüllkurve dargestellt (Fig. 9.1).

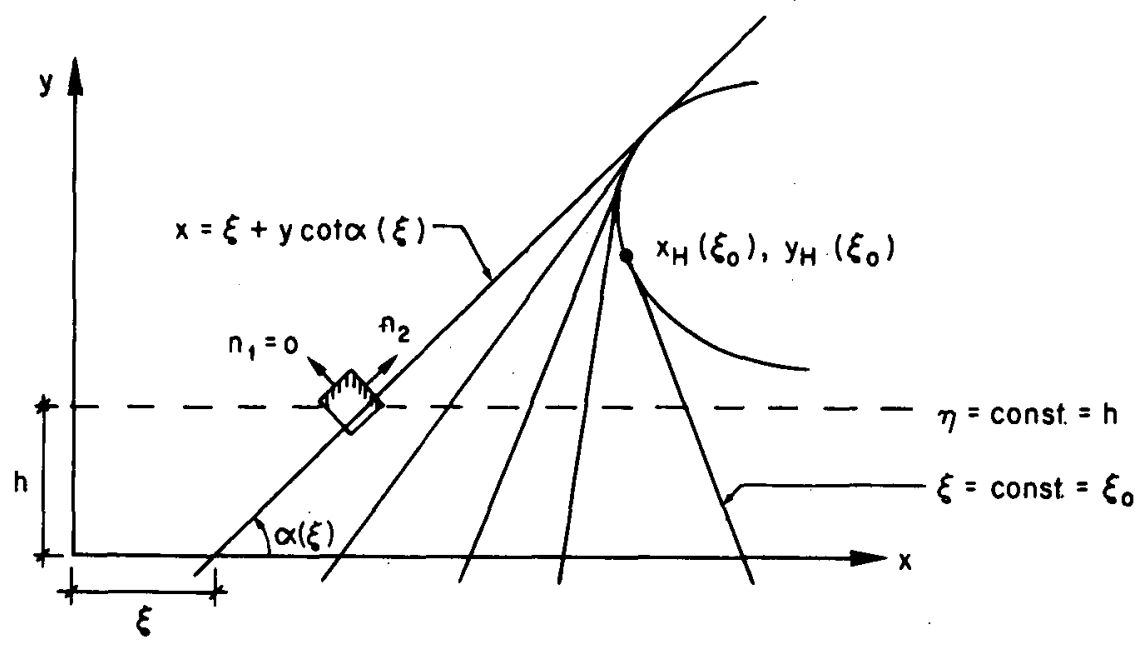

Fig. 9.1 Koordinatensysteme und Betondrucktrojektorienschor

Die Tangenten an die Raumkurve bzw. ihre Projektionen sind die Charakteristiken der parabolischen Differentialgleichung (9.6). Entlang ihnen lassen sich beliebige, abwickelbare Flächen zusammensetzen, und über sie hinweg kann eine Lösung nur unter Zuzug zusätzlicher Randbedingungen fortgesetzt werden. Als Richtung der verschwindenden Hauptkrümmung von $F$ sind sie aber auch Richtung der Betondrucktrajektorien. Die Betondrucktrajektorien im Fliessregime I bilden also, abgesehen von den Lösungen mit konstantem tana ( F Zylinderfläche), zentrierte und unzentrierte Fächer ( $F$ Kegel bzw. allgemeine abwickelbare Fläche).

Die Gleichung der Drucktrajektorienschar lautet, wenn als Parameter der Geradenschar der Achsabschnitt $\xi$ auf der $x$-Achse eingeführt wird (Fig. 9.1)

$\left.\begin{array}{l}x=\xi+y k(\xi), \\ \text { mit } k(\xi)=\cot \alpha(\xi) .\end{array}\right\}$

Die Parameterdarstellung der Hülikurve wird durch Nullsetzen der Ableitung nach dem Parameter $\xi$ gewonnen. 


$$
\left.\begin{array}{rl}
x_{, \xi}=0 & =1+y_{H} k^{\prime}(\xi), \\
y_{H} & =-\frac{1}{k^{\prime}(\xi)}, \\
x_{H} & =\xi-\frac{k(\xi)}{k^{\prime}(\xi)},
\end{array}\right\}
$$

Zur Lösung konkreter Probleme ist der Ansatz mit der Spannungsfunktion nicht geeignet. Die vorstehenden Ausführungen zeigen jedoch, dass ein brauchbarer Formelapparat zur Bestimmung der Spannungsfelder aus den Randbedingungen gewonnen werden kann, wenn als Unbekannte die Charakteristiken-bzw. Drucktrajektorienrichtung $\alpha$ und die nicht verschwindende Betonhauptspannung $\mathrm{n}_{2}$ eingeführt werden. Mit

$$
\begin{aligned}
& n_{x}=n_{2} \cos ^{2} \alpha \\
& n_{y}=n_{2} \sin ^{2} \alpha \\
& n_{x y}=n_{2} \sin \alpha \cos \alpha,
\end{aligned},
$$

ist (9.4) identisch erfült. Einsetzen in (9.3) ergibt das parabolische Differentialgleichungssystem

$n_{x, x}+n_{x y, y}=n_{2}(-\alpha, x \sin 2 \alpha+\alpha, y \cos 2 \alpha)+n_{2, x} \cos ^{2} \alpha+n_{2, y} \sin \alpha \cos \alpha=0$,

$n_{x y, x}+n_{y, y}=n_{2}(\alpha, x \cos 2 \alpha+\alpha, y \sin 2 \alpha)+n_{2, x} \sin \alpha \cos \alpha+n_{2, y} \sin ^{2} \alpha=0$.

Multiplikation von (9.11) mit cota und Subtraktion von (9.10) ergibt

$\alpha, x \cot \alpha+\alpha, y=0$

Die Lösungsmannigfaltigkeit dieser quasi-linearen homogenen partiellen Differentialgleichung 1. Ordnung für $\alpha$ ist in impliziter Form gegeben durch [8]

$\alpha=f(x-y \cot \alpha)$,

oder gleichwertig

$\cot \alpha=k(x-y \cot \alpha)$,

wobei $f$ bzw. K willkürliche Funktionen sind, die aus den Anfangswerten von $\alpha$ längs z.B. y $=0$ zu bestimmen sind. Dabei wird von den Koeffizienten der Differentialgleichung (9.12), d.h. von cota und von den Anfangswerten stetige Differenzierbarkeit vorausgesetzt. Werden anstelle von $x, y$ die neuen Variablen

$\xi=x-y \cot \alpha$,

$n=y$

eingeführt, geht (9.13) über in

$\alpha=f(\xi)$,

$\cot \alpha=k(\xi)$ 
Längs der Kurven $\xi=$ const. ist $\alpha$ bzw. cota konstant und diese sind folglich die charakteristischen Grundkurven der homogenen Differentialgleichung (9.12) und damit auch des parabolischen Systems (9.10), (9.11). Tatsächlich stellt die wegen (9.15b) implizite Definitionsgleichung (9.14a) wieder die einparametrige Geradenschar der Betondrucktrajektorien dar (Fig. 9.1)

$x=\xi+y k(\xi)$.

Sind die Variablen eines parabolischen Differentialgleichungssystems in einem Punkt 0 durch Randbedingungen gegeben, so ist ihr Wert in allen Punkten der charakteristischen Kurve durch 0 eindeutig bestimmt.

Durch die Einführung des Parameters $\xi$ der Charakteristikenschar und einer beliebig wählbaren zweiten Funktion $n$ als neue unabhängige Variablen (9.14) gelingt es somit, die Differentialgleichung ( 9.10 ) oder (9.11) über $n$, d.h. entlang der charakteristischen Geraden $\xi=$ const. zu integrieren und $n_{2}$ als Funktion von $\xi$, $n$ und $k^{\prime}(\xi)$ auszudrücken. Durch Einsetzen von längs Kurven $\eta(\xi)$ in $\xi$ und cota $=k(\xi)$ ausdrückbaren Randbedingungen erhält man schliesslich eine einzige gewöhnliche Differentialgleichung für $k(\xi)$. Es würde näher liegen, als Kurven $n=$ const. die Betonzugtrajektorien einzuführen. Da jedoch Stahlbetonscheiben meist rechteckig sind, und Randbedingungen vielfach entlang Rändern y = const. zu formulieren sind, wird die Wahl (9.14b) vorgezogen. Zudem ergibt sich damit eine vollständig analoge mathematische Formulierung wie für die Regimes III bis VII, bei welchen wegen des hyperbolischen Charakters der Differentialgleichung keine Wahlfreiheit für $n$ besteht.

Zur Variablentransformation werden noch die Abteilungen von $\xi$ und $\eta$ nach $x$ und $y$ benötigt. Ableiten von (9.14) nach $x$ und $y$ unter Beachtung von (9.15b) ergibt

$$
\begin{aligned}
& \xi_{, x}=\frac{1}{1+n k^{\prime}(\xi)}, \\
& \xi_{, y}=-\frac{k(\xi)}{1+n k^{\prime}(\xi)}, \\
& \eta_{, x}=0, \quad \eta_{, y}=1, \\
& k(\xi) \xi_{, x}+\xi_{, y}=0 .
\end{aligned}
$$

Die letzte Beziehung folgt natürlich mit (9.15a,b) auch sofort aus (9.12). Schliesslich folgt aus (9.15) noch

$$
\begin{aligned}
& k^{\prime}(\xi)=(\cot \alpha), \alpha,{ }^{\alpha}, \xi \\
& \alpha, \xi \quad=f^{\prime}(\xi)=-\sin ^{2} \alpha k^{\prime}(\xi) .
\end{aligned}
$$

Einsetzen von (9.12) in den Klammerausdruck von (9.10) und Ausdrücken der Ableitungen nach $x$ und $y$ durch solche nach $\xi$ und $n$ unter beachtung von (9.15) bis (9.20) ergibt nach einiger Rechnung

$$
\frac{n_{2, n}}{n_{2}}=-\frac{k^{\prime}(\xi)}{1+n k^{\prime}(\xi)} \text {. }
$$


Durch Integration über $n$ erhält man nun sofort die gesuchte Beziehung für $n_{2}$

$n_{2}=g(\xi) /\left(1+n k^{\prime}(\xi)\right)$,

wobei g die noch von $\xi$ abhängige "Integrationskonstante" ist.

Die gemäss Fig. 9.1 zu erwartende physikalische Bedeutung von (9.22) verifiziert man sofort durch Einsetzen van (9.8) in (9.22):

$n_{2}=g(\xi) \frac{1}{1-y / y_{H}(\xi)}=g(\xi) \frac{y_{H}(\xi)}{y_{H}(\xi)-y}$

Längs einer Trajektorie $\xi$ = const. verläuft die Betondruckspannung umgekehrt proportional zum Abstand des Berührungspunktes von Trajektorie und Hüllkurve.

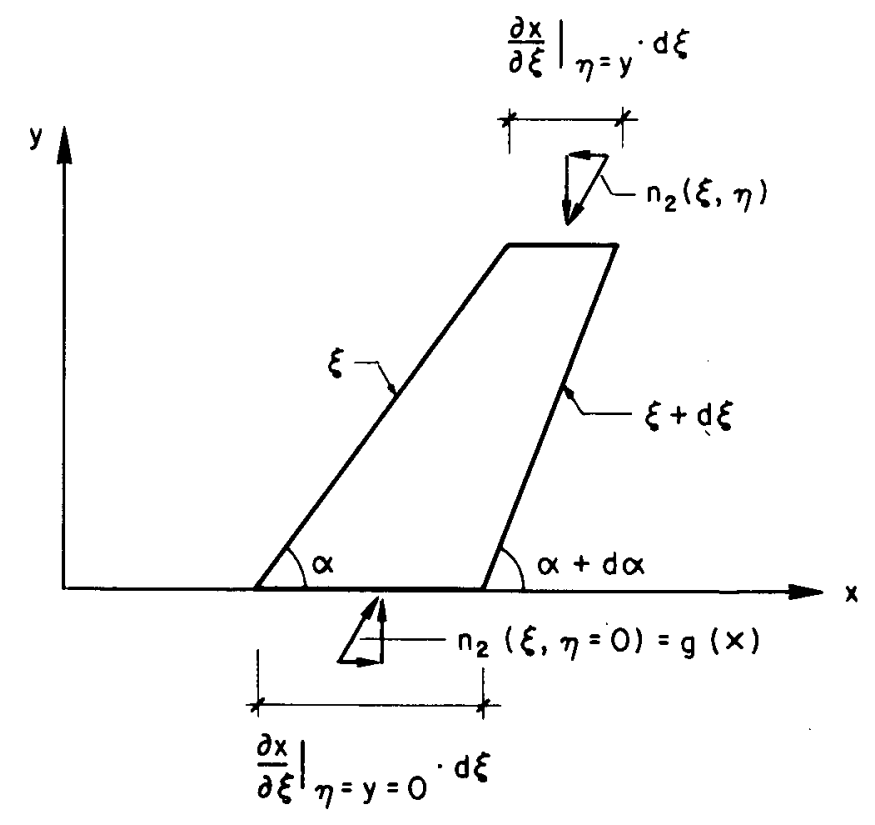

Fig. 9.2 Direkte Ableitung von (9.22)

Fig. 9.2 zeigt, dass (9.22) auch sofort aus dem Gleichgewicht eines differentiellen Streifens

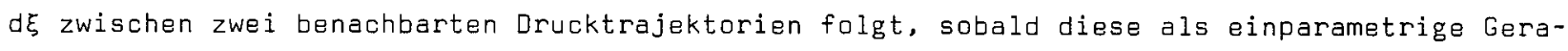
denschar bekannt sind. Mit

$x=\xi+n \cot \alpha=\xi+n k(\xi)$,

$x_{, \xi}=\frac{\partial x}{\partial \xi}=1+n k^{\prime}(\xi)$,

und wenn $n_{2}$ längs $n=y=0 z u g(\xi)=g(x)$ vorgegeben ist, ergibt das vertikale Gleichgewicht

$n_{2} \sin ^{2} \alpha\left(1+n k^{\prime}(\xi)\right) d \xi=g(\xi) \sin ^{2} \alpha d \xi$,

$n_{2}=g(\xi) /\left(1+n k^{\prime}(\xi)\right)$. 
Beispiele zur Bestimmung von $k(\xi)$ und $g(\xi)$ aus den Randbedingungen finden sich in den $A b-$ schnitten 10 und 11 .

In einem vollplastifizierten Gebiet, in dem sich der Spannungszustand ausschliesslich im Regime $C-D$, d.h. auf der Kante $C-D$ zwischen den Fliessregimes I und III, IV, V (Fig. 3.2) befindet, gilt zudem (Fig. 2.6)

$n_{2}=-P_{b}$,

$g(\xi)=-P_{b}\left(1+\eta k^{\prime}(\xi)\right)$.

Da die rechte Seite (9.25) nicht Funktion von $\eta$ sein darf, folgt sofort

$k^{\prime}(\xi)=0, k(\xi)=\cot \alpha=$ const.,

und der Spannungszustand kann nur konstant sein. Dies gilt natürlich auch für alle anderen Regimes, in welchen der Beton in einem einaxialen Druckspannungszustand an der Betondruckfestigkeitsgrenze liegt, und beide Armierungen auf Zug oder Druck fliessen, d.h. für alle Kanten der Fliessfiguren $2.4,2.5$.

Die zum Fliessregime II (Fig. 2.6) gehörenden zweiaxialen Betondruckspannungszustände $-P_{b} \leq n_{1} \leq 0, n_{2}=-P_{b}$ können als Superposition eines "hydrostatischen" Druckspannungszustandes $\bar{n}_{1}=\bar{n}_{2}=-P_{b}$ und eines einaxialen Zugspannungszustandes $n_{1}^{*}=n_{1}+P_{b}, n_{2}^{*}=0$ aufgefasst werden. Da der erstere weder $\forall i e$ Gleichgewichtsbedingungen noch die Hauptspannungsrichtung beeinflusst und die Beziehungen (9.13), (9.22) unabhängig vom Vorzeichen des einaxialen Spannungszustandes gelten, ist gleichzeitig auch die allgemeinste form der Betonspannungsfelder im Regime II gewonnen. a bezeichnet dann die Richtung der absolut kleineren Betonhauptspannung $n_{1}$, während in (9.22) $n_{2}$ durch $n_{1}^{*}=n_{1}+P_{b}$ zu ersetzen ist. Mit der Fliessfigur 2.5 ist die Erhöhung der zweiaxialen Betondruckfestigkeit gegenüber der einaxialen vernachlässigt. Eine Verwendung obiger Spannungsfelder kann jedoch wegen ihrer Einfachheit trotzdem sinnvoll sein, wenn statisch zulässige Spannungsfelder gesucht sind zur Gewinnung unterer Grenzwerte oder zur Lösung des Fortsetzungsproblems in starren Gebieten.

\subsection{Geschwindigkeitsfelder im Regime I}

Im folgenden werden plastifizierte Gebiete betrachtet, in welchen die Betondruckfestigkeit nicht erreicht ist, und die sich somit in den Fliessregimes I ader A befinden. Ein Geschwindigkeitsfeld u,v (Fig. 9.3) ist mit dem Regime I (exkl. A) verträglich, wenn es der kinematischen Bindung $\dot{\varepsilon}_{1} \geq \dot{\varepsilon}_{2}=0$ genügt. Die letzte Gleichung ergibt, wenn die parametrische Form (6.5) zugrunde gelegt wird und die Dehnungsgeschwindịgkeiten durch u,v ausgedrückt werden, das schon in Abschnitt 6.4 verwendete Differentialgleichungssystem (9.27). Dieses ist natürlich nur dann nicht unterbestimmt, wenn $\alpha$ als Funktion von $x, y$ vorgegeben ist. Es ist von parabolischem Typ mit der charakteristischen Richtung $\alpha$

$\left.\begin{array}{rl}v, x+u, y+2 u, x \cot \alpha & =0, \\ v, y-u, x \cot ^{2} \alpha & =0 .\end{array}\right\}$

Ein Geschwindigkeitsfeld, das (9.27) erfüllt, ist mit einem (9.1), (9.2) genügenden Spannungsfeld verträglich, wenn die Neigungen a der kleineren Hauptdehnungsgeschwindigkeit $\dot{\varepsilon}_{2}=0$ und 


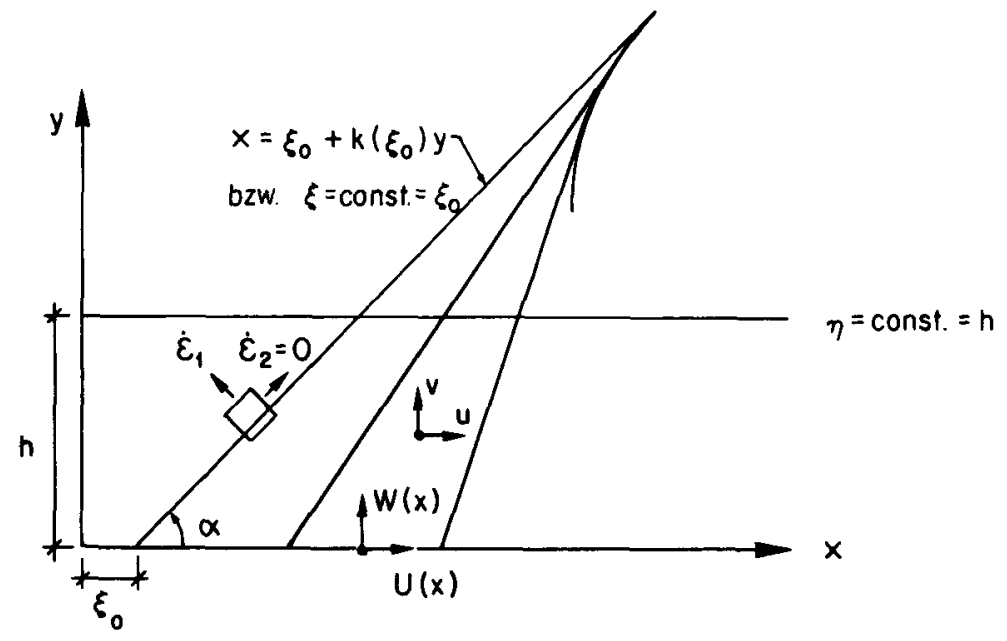

Fig. 9.3 Koordinatensysteme und $\dot{\varepsilon}_{2}$ - Trajektorienschor

der kleineren Betonhauptspannung $n_{2}$ übereinstimmen. Das zur Fliessbedingung (2.7 I) gehörende ebene Spannungsproblem ist somit durch die vier Differentialgleichungen (9.10), (9.11), (9.27) für die vier Unbekannten $n_{2}, \alpha, u, v$ definiert. Seine Lösungen können nur Geschwindigkeitsfelder enthalten, die (9.12), (9.13) genügen und somit ebenfalls gerade Trajektorien der verschwindenden Hauptdehnungsgeschwindigkeit $\dot{\varepsilon}_{2}=0$ aufweisen (Fig. 9.3). Für die Trajektorienfelder (9.13) kann (9.27) durch den Uebergang auf die Variablen (9.14) sofort integriert werden, und $u, v$ lassen sich wie in Abschnitt 6.4 durch zwei willkürliche aus den Randbedingungen zu bestimmende Funktionen $f(\xi), g(\xi)$ und $\cot \alpha=k(\xi)$ ausdrücken.

Die Wahl der abhängigen und unabhängigen Variablen u,v, $n$ erfolgte wieder im Hinblick auf die Tatsache, dass bei Stahlbetonscheiben Randbedingungen normalerweise entlang zur Armierung parallelen Rändern und in zur Armierung parallelen Geschwindigkeitskomponenten zu formulieren sind.

Drückt man die Ableitungen nach $x$ und $y$ in (9.27) mittels der Beziehungen (9.14) bis (9.19) durch solche nach $\xi$, $n$ aus, ergibt sich nach einiger Rechnung

$v, n+k(\xi) u, n=0$,

$\left(v, \xi+k(\xi) u, \xi^{\prime}+u, \eta\left(1+\eta k^{\prime}(\xi)\right)=0\right.$.

Durch Elimination von $v$ aus (9.28) durch Ableiten nach $\xi$ bzw. $n$ verifiziert man für nicht auf der Hüllkurve liegende Punkte sofort den parabolischen Charakter des Differentialgleichungssystems: $u, \eta \eta=0$. Insbesondere sind die Geraden $\xi$ = const. auch die charakteristischen Kurven des Geschwindigkeitsfeldes.

Die Differentialgleichung (9.28a), die die Undehnbarkeit der $\dot{\varepsilon}_{2}$-Trajektorien ausdrückt, kann unmittelbar integriert werden,

$v+k(\xi) u=f(\xi)$,

und es folgt

$v_{, \xi}+k(\xi) u, \xi=f^{\prime}(\xi)-k^{\prime}(\xi) u$. 
Einsetzen von (9.30) in (9.28b), Umordnen und Integration ergibt

$$
\begin{aligned}
& \frac{u, \eta+f^{\prime}(\xi)}{u+n f^{\prime}(\xi)=} \frac{k^{\prime}(\xi)}{1+n k^{\prime}(\xi)}, \\
& u+n f^{\prime}(\xi)=g(\xi)\left(1+\eta k^{\prime}(\xi)\right), \\
& u=\quad g(\xi)\left(1+n k^{\prime}(\xi)\right)-n f^{\prime}(\xi), \\
& v=f(\xi)-k(\xi)\left\{g(\xi)\left(1+n k^{\prime}(\xi)\right)-n f^{\prime}(\xi)\right\} .
\end{aligned}
$$

Der Term $\left(1+\eta k^{\prime}(\xi)\right)$ kann mit $(9.8)$ auch durch

$\left(1+\eta k^{\prime}(\xi)\right)=\left(1-y / y_{H}(\xi)\right)$

ausgedrückt werden, und ist entlang einer Trajektorie $\xi$ = const. proportional zum Abstand ihres Berührungspunktes mit der Hüllkurve.

Für cota $=k(\xi)=$ const. geht (9.33) wieder über in (6.32). Formuliert man wie in Abschnitt 6.4 längs $y=\eta=0$ die Randbedingung $u(y=0)=U(x), v(y=0)=W(x)$, so verschwindet der Term (9.34) auch für variables $\alpha(\xi)$, und die Geschwindigkeiten $u, v$ sind wieder durch (6.34) gegeben. Die Beziehungen (6.34) gelten somit allgemein, wenn dort cota als $\cot \alpha(\xi)=k(\xi)$ aufgefasst wird. Die Funktionen $g(\xi), f(\xi)$ bzw. $U(x), W(x)$ sind nur soweit frei wählbar, als die Bedingung positiver Dehnungsgeschwindigkeiten

$\dot{\varepsilon}_{x}=u, \xi_{, x}=u, \xi^{\prime\left(1+\eta k^{\prime}(\xi)\right) \geq 0}$

eingehalten wird.

In Fig. 9.4 a,b ist der Verlauf der Geschwindigkeiten u,v infolge $g(\xi)$ bzw. $f(\xi)$ entlang einer Betondrucktrajektorie dargestellt. Das Geschwindigkeitsfeld setzt sich zusammen aus einer entlang der Drucktrajektorie linearen, zu ihr senkrechten Geschwindigkeitskomponente und einer reinen Translation der Trajektorie. Drucktrajektorien bleiben während der Verschiebung unverformt. Da sie auch senkrecht zu den verformten Zugtrajektorien bleiben müssen, kann man als Vorstellungshilfe einen gekrümmten Stab einführen, dessen Stabaxe mit einer Zugtrajektorie und dessen Querschnitte mit den Drucktrajektorien zusammenfallen. Die Geschwindigkeitsfelder (9.33) entsprechen dann denjenigen dieses Stabes unter der Voraussetzung eben. ungedehnt und senkrecht zur Stabaxe bleibender Querschnitte.

Die Bahnlinien infolge $g(\xi)$ haben die Hüllkurve als gemeinsame Evolute und fallen mit den Zugtrajektorien zusammen. Die Verzerrungsgeschwindigkeiten infolge $g(\xi)$ sind gemäss (9.35), (9.33) konstant über $n$. solange $k^{\prime \prime}(\xi)=0$ gilt, d.h. solange die Drucktrajektorien parallel sind $\left(k^{\prime}=0\right.$, "gerader Stab") oder einen zentrierten Fächer bilden ( $k^{\prime}$ = const., "Kreisstab"). Die Funktion $g(\xi)$ entspricht daher im wesentlichen der Verlängerung des als Vorstellungshilfe eingeführten, gekrümmten Stabes, während $f(\xi)$ und $f^{\prime \prime}(\xi)$ mit dessen Durchbiegung bzw. Krümmung zu assoziieren sind. Die Verzerrungsgeschwindigkeiten infolge $f^{n}(\xi)$ verlaufen gemäss (9.35), (9.33) schon für den zentrischen Fächer nicht mehr linear entlang einer Drucktrajektorie. Dies entspricht dem Verlauf der Dehnungen über den Querschnitt eines biegebeanspruchten Kreisstabes. Dagegen verlaufen die Geschwindigkeitssprünge infolge Unstetigkeiten in $g(\xi)$ und $f^{\prime}(\xi)$ ("Kollapsrisse") immer linear entlang einer Betondrucktrajektorie. 


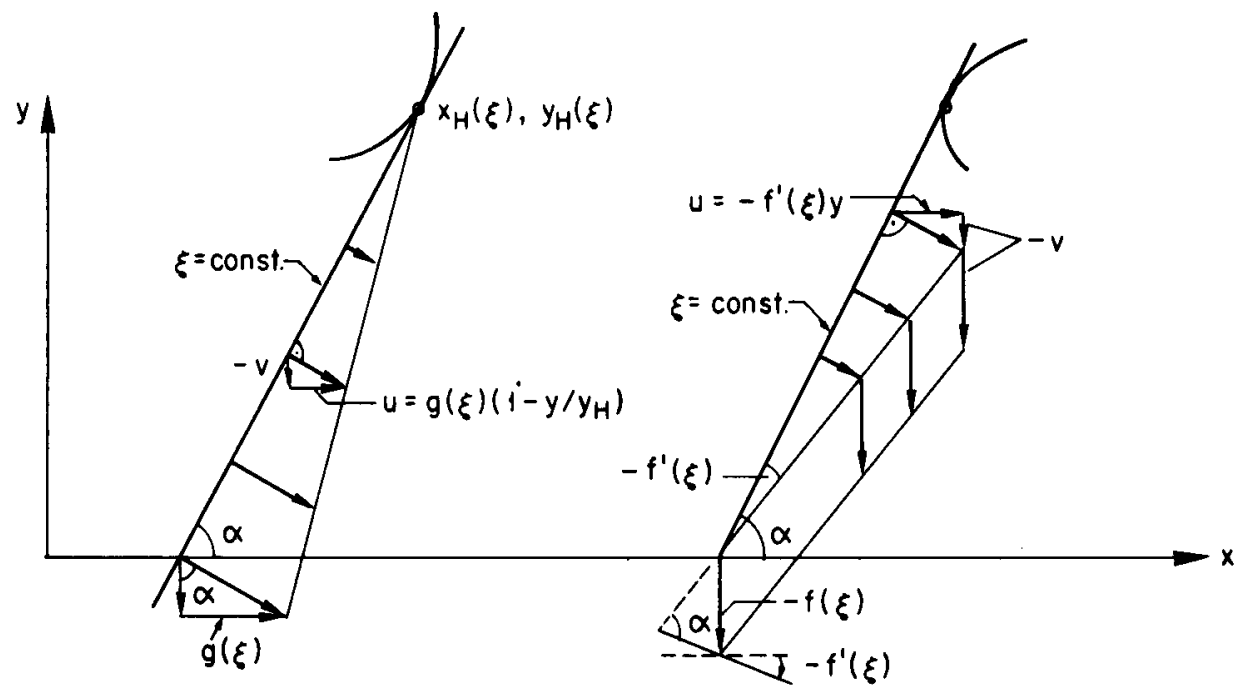

$\begin{array}{ll}\text { (a) } u, v \text { infolge } g(\xi) & \text { (b) } u, v \text { infolge } f(\xi)\end{array}$

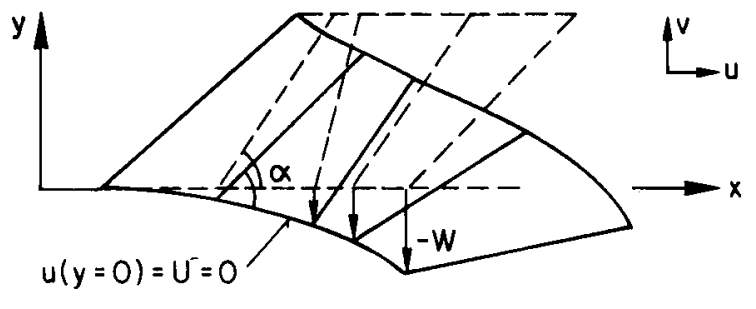

(c) "Kamm" - Mechanismus

Fig. 9.4 Geschwindigkeitsfelder (9.33)

Wählt man als Bezugsachse $y=\eta=0$ eine infolge Nichtfliessens der Längsarmierung oder des Flansches ungedehnte Faser $(U=g=0)$, so vereinfachen sich die Beziehungen (6.34), (9.33) wesentlich, und es bleiben, wie Fig. 9.4 b zeigt, auch die schiefen winkel $\alpha$ zwischen Drucktrajektorie und Bezugsachse erhalten. Fig. 9.4 c zeigt einen solchen "kammartigen" Mechanismus.

Es sei betont, dass (9.33) nicht alle Geschwindigkeitsfelder enthält, die $\dot{\varepsilon}_{1} \geq \dot{\varepsilon}_{2}=0$ genügen. Das Differentialgleichungssystem (9.27) wurde nur für die Trajektorienfelder (9.13) gelöst, die aus rein statischen Ueberlegungen gewonnen wurden. Dies ist vor allem dann zu beachten, wenn a priori nur mit einer aus der Fliessfläche I bestehenden Fliessfigur gearbeitet wird. Die kinematische Bindung $\dot{\varepsilon}_{1} \geq \dot{\varepsilon}_{2}=0$ kann dann als kinematische Zulässigkeitsbedingung aufgefasst werden wie eine Inkompressibilitätsbedingung. Bei der Mechanismusmethode besteht dann kein Grund, Felder mit gekrümmten $\dot{\varepsilon}_{2}$-Trajektorien auszuschliessen.

In exakten Lösungen können solche Felder jedoch nur in Gebieten auftreten, wo die Betonspannungen verschwinden, und wo sich der Spannungszustand somit im Fliessregime $A$ an der Zugkegelspitze befindet. Mit dem Regime $A$ sind aber alle Geschwindigkeitsfelder verträglich, die $\dot{\varepsilon}_{1} \geq \dot{\varepsilon}_{2} \geq 0$ genügen. 
In den Regimes IV-VII (Fig. 2.6) befindet sich der Beton in einem einaxialen Spannungszustand an der Druckfliessgrenze, $n_{1}=0, n_{2}=-P_{b}$, und der einzige Freiheitsgrad ist die Drucktrajektorienrichtung $\alpha$. In der Armierung liegt die Spannung jeweils einer Armierungsrichtung an der Zug- oder Druckfliessgrenze und beeinflusst die Gleichgewichtsbedingungen nicht, wenn wieder die Konstanz von $P_{x}$ in $x$ - und $P_{y}$ in $y$-Richtung vorausgesetzt wird. Die Gleichgewichtsbedingung (9.1) in Richtung der fliessenden Armierung liefert somit die gesuchte Beziehung für die mögliche Variation von $\alpha$, während der Spannungsverlauf in der nicht massgebenden Armierung aus der zweiten Gleichgewichtsbedingung folgt.

Die Resultate für die im folgenden betrachteten Fliessregimes V/VII mit auf Zug bzw. Druck fliessender y-Armierung können sofort durch Vertauschen der Achsen auf die Regimes IV/VI übertragen werden. Da die Gleichgewichtsbedingungen unbeeinflusst davon bleiben, ob die Armierung auf Zug oder Druck fliesst, können die Regimes $V$ und VII ebenfalls gemeinsam behandelt werden.

In einem ausschliesslich im Regime III plastifizierten Gebiet fliesst keine der beiden Armierungen. Da jedoch der Betonspannungszustand konstant ist, $n_{1}=0, n_{2}=-P_{b} \alpha= \pm 45^{\circ}$ (orthogonale Armierung), muss die Stahlspannung entlang der Armierung ebenfalls konstant sein.

Mit

$\left.\begin{array}{l}n_{x}=n_{2} \cos ^{2} \alpha, \\ n_{y}=n_{2} \sin ^{2} \alpha, \\ n_{x y}=n_{2} \sin \alpha \cos \alpha, \\ n_{z}=-p_{b},\left|z_{y}\right|=p_{y} .\end{array}\right\}$

sind die Fliessbedingungen (2.7 V) bzw. (2.7 VII) identisch erfüllt (wobei in (2.7 VII) die Druckfliessspannung $-P_{y}$ vernachlässigt wurde). Mit (2.1) und (9.36) lauten die Scheibengleichgewichtsbedingungen (9.1)

$\left.\begin{array}{l}n_{x, x}+n_{x y, y}=n_{2}(-\alpha, x \sin 2 \alpha+\alpha, y \cos 2 \alpha)=-z_{x, x}, \\ n_{x y, x}+n_{y, y}=n_{2}(\alpha, x \cos 2 \alpha+\alpha, y \sin 2 \alpha)=0 .\end{array}\right\}$

Das Differentialgleichungssystem (9.37) ist von hyperbolischem Typ und besitzt - wie später verifiziert wird - die zwei Charakteristikenscharen $d x=\cot 2 \alpha$ dy und $d y=0$. Die erste Schar stellt Kurven der Neigung tan $2 \alpha$, die zweite Parallelen zur nicht fliessenden Armierung dar.

Mit der zweiten Gleichung (9.37) ist wieder eine homogene, quasilineare partielle Differentialgleichung .1. Ordnung für die Drucktrajektorienneigung $\alpha$ gefunden

$\alpha_{, x} \cot 2 \alpha+\alpha_{, y}=0$

mit der in impliziter form gegebenen Lösungsmannigfaltigkeit

$\cot 2 \alpha=K(x-y \cot 2 \alpha)$ 
Dabei bedeutet $K$ wieder eine willkürliche, aus den Randbedingungen zu bestimmende Funktion. Die Kurven konstanten Winkels a und Charakteristiken von (9.38) sind offensichtlich wieder eine einparametrige Geradenschar, deren Gleichung sich wie früher in der form

$x=\xi+y k(\xi)$

schreiben lässt, wenn

$\left.\begin{array}{l}\xi=x-y \cot 2 \alpha \quad, n=y, \\ \text { mit } \quad \cot 2 \alpha=k(\xi)\end{array}\right\}$

gesetzt wird. Damit ist auch die erste Charakteristikenschar des systems (9.37) als Geradenschar $\xi$ = const. verifiziert. Der Betonspannungszustand ist also wieder sehr einfach. Wohl sind die Drucktrajektorien gekrümmt, aber Punkte gleicher Trajektorienneigung $\alpha$ liegen auf Geraden der Neigung $2 \alpha$, die eine einparametrige Geradenschar bilden (Fig. 9.5).

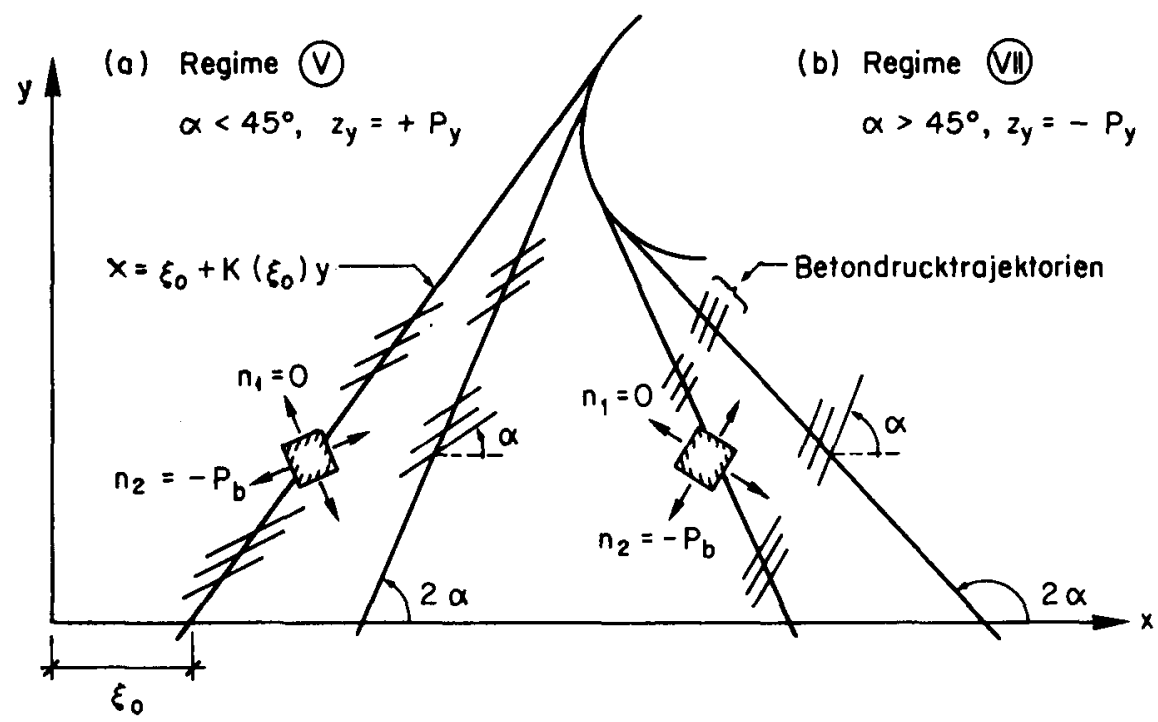

Fig. 9.5 Spannungsfelder der Regimes V, VII

Dabei gehören Betonspannungszustände mit $0 \leq|\alpha| \leq 45^{\circ}$ zum Regime $V$ mit auf Zug fliessender $y$-Armierung (Fig. 9.5 a), solche mit $45^{\circ} \leq|\alpha| \leq 90^{\circ}$ zum Regime VII mit an der Druckfliessgrenze liegender y-Armierung (Fig. 9.5 b).

Einsetzen von (9.38) in die erste Gleichung (9.37) und Uebergang auf die Variablen $\xi$, $n$ ergibt

$\left.\begin{array}{l}z_{x, x}=n_{2} \frac{\alpha, x}{\sin 2 \alpha}=-P_{b} \frac{\alpha, x}{\sin 2 \alpha}, \\ z_{x, \xi}=-P_{b} \frac{\alpha, \xi}{\sin 2 \alpha}\end{array}\right\}$

Wegen $\alpha=\alpha(\xi)$ gilt offenbar $z_{x, \xi \eta}=0$, und damit ist sowohl der hyperbolische Charakter des Gesamtsystems (9.37) als auch die zweite Charakteristikenschar $\eta$ = const. verifiziert. Integration von (9.42) über $\xi$ ergibt

$d z_{x}=-P_{b} \frac{d \alpha}{\sin 2 \alpha}=\frac{1}{2} P_{b} \frac{d(\cot \alpha)}{\cot \alpha}$, 
$z_{x}=\frac{1}{2} P_{b} \ln (\cot \alpha(\xi))+f(\eta)$

Die primär interessierende Spannungsänderung in x-Richtung ist also zwischen zwei Charakteristiken $\xi=\xi_{0}$ und $\xi=\xi_{1}$ konstant (Fig. 9.6). Die Spannungsänderung (Zug + ) ist dabei gemäss (9.43) positiv in Richtung zunehmendem cota, d.h. abnehmendem $\alpha(0 \leq \alpha \leq 90)$. Die Spannung selbst kann sowohl Zug- als auch Druckspannung sein (Term $f(\eta))$.

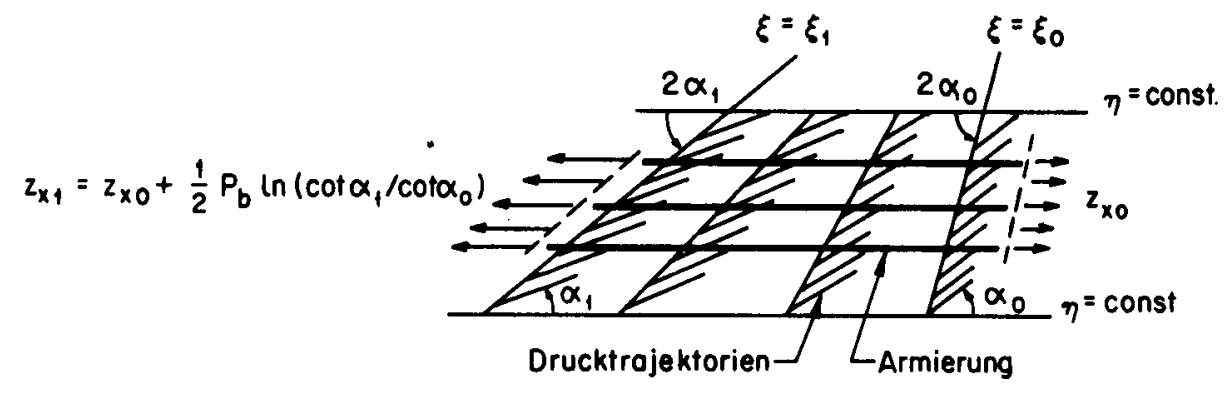

Fig. 9.6 Beanspruchung der nicht fliessenden Armierung

\subsection{Geschwindigkeitsfelder in den Regimes III-VII}

Im folgenden werden plastifizierte Gebiete betrachtet, in welchen die $x$-Armierung nicht fliesst, und die sich somit in den Fliessregimes V, III oder VII befinden. Die Resultate sind natürlich sofort wieder auf Gebiete mit nicht fliessender y-Armierung übertragbar.

Ein Geschwindigkeitsfeld $u, v$ ist mit den Regimes $V$, VII verträglich, wenn es der kinematischen Bindung $\dot{\varepsilon}_{x}=u_{, x}=0$ genügt und somit von der form $u=f(y), v=g(x, y)$ ist.

Ein Geschwindigkeitsfeld, für das $u_{, x}=0$ gilt, ist mit einem (9.37) genügenden Spannungszustand verträglich, wenn die Neigungen $\alpha$ der kleineren Hauptdehnungsgeschwindigkeit $\dot{\varepsilon}_{2}$ und der kleineren Betonhauptspannung $n_{2}$ übereinstimmen. Ist $\alpha$ als Funktion von $x, y$ bekannt, so genügen $u, v$ dem hyperbolischen Differentialgleichungssystem (9.45) mit den charakteristischen Kurven $d x=\cot 2 \alpha d y$ und $d y=0$.

$\left.\begin{array}{l}(u, y+v, x) \cot 2 \alpha+v, y=0, \\ u_{, x}=0 .\end{array}\right\}$

Das zur Fliessbedingung (2.7 V) bzw. (2.7 VII) gehörende ebene Spannungsproblem der plastifizierten Scheibe ist also durch die vier Differentialgleichungen (9.37), (9.45) für die vier Variablen $z_{x}, \alpha, u, v$ definiert. Seine Lösungen können nur Geschwindigkeitsfelder enthalten, die (9.38), (9.39) genügen. Für die Trajektorienfelder (9.39) lässt sich (9.45) sofort integrieren, wenn auf die Variablen $\xi$, $n$ (9.41) übergegangen wird. Die Ableitungen der neuen nach den alten Variablen sind dabei wieder durch (9.16) bis (9.19) gegeben, wenn dort $k l e i n k(\xi)$ $=\cot \alpha$ durch gross $K(\xi)=\cot 2 \alpha$ ersetzt wird.

Ausdrücken der Ableitungen nach $x, y$ in (9.45) durch solche nach $\xi$, $n$ mittels (9.41) und ( 9.16 ) bis (9.19) ergibt unter Ausschiuss des uninteressanten Falls $\alpha=0$ 
$\left.\begin{array}{rl}v, n+k(\xi) u, n & =0, \\ u, \xi & =0 .\end{array}\right\}$

Eliminiert man aus der ersten Gleichung (9.46) u durch Auflösen nach $u$, und Ableiten nach $\xi$, folgt sofort der hyperbolische Charakter von (9.45): $v, \xi n+\ldots=0$. Insbesondere sind $\xi=$ const., $n=$ const. auch die Charakteristiken des Geschwindigkeitsfeldes.

Beide Gleichungen (9.46) können unmittelbar integriert werden und ergeben formal das gleiche Resultat wie in Abschnitt 7.2 mit dem einzigen Unterschied, dass nun $\cot 2 \alpha=k(\xi)$ variabel ist.

$\left.\begin{array}{l}u=g(\eta) \\ v=f(\xi)-g(\eta) k(\xi), \\ \text { mit } k(\xi)=\cot 2 \alpha .\end{array}\right\}$

Die Dehnungsgeschwindigkeit in y-Richtung beträgt

$\dot{\varepsilon}_{y}=v, y=f^{\prime}(\xi) \xi, y-g^{\prime}(n) K(\xi)-g(n) K^{\prime}(\xi) \xi, y$,

$\dot{\varepsilon}_{y}=-\cot 2 \alpha\left\{\frac{f^{\prime}}{1-y / y_{H}}+\left(g^{\prime}+\frac{g}{y_{H}-y}\right)\right\}$,

wenn (9.17) und (9.8) beachtet werden. Mit (7.30) sind auch die übrigen Verzerrungsgeschwindigketen bekannt.

Die Geschwindigkeitsfelder (9.47) sind in Fig. 9.7 veranschaulicht und zwar in Fig. 9.7 a,b solche infolge $f(\xi)$ und in Fig. $9.7 \mathrm{c}, d$ solche infolge $g(\eta)$. Die Schraffur gibt jeweils die Drucktrajektorienrichtung $\alpha$ an $\left(-90^{\circ} \leq \alpha \leq 90^{\circ}\right)$. Fig. 9.7 a.c zeigt Mechanismen im Regime $V$ $\left(0 \leq|\alpha| \leq 45^{\circ}, \dot{\varepsilon}_{y} \geq 0\right)$, Fig. 9.7 b, d solche im Regime VII $\left(45^{\circ} \leq|\alpha| \leq 90^{\circ}, \dot{\varepsilon}_{y} \leq 0\right)$. Dabei sind übersichtlichkeitshalber immer Mechanismen gezeichnet, die zu positivem $\alpha$ führen.

Für die Felder infolge $f(\xi)$ sind die Charakteristiken $\xi=$ const. wie bei konstantem $\alpha$ Orte konstanter Durchbiegung $\checkmark$ und konstanter Durchbiegungssprünge $\Delta v$. Dagegen verlaufen die Verzerrungsgeschwindigkeiten infolge $f(\xi)$ entlang $\xi$ = const. nun umgekehrt proportional zum Abstand von der Hüllkurve.

Die Bahnlinien der Geschwindigkeitsfelder infolge $g(\eta)$ haben als gemeinsame Evolute die Hüll-

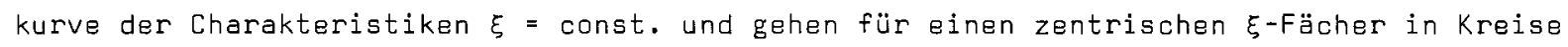
über. Die einzelnen Charakteristiken $\eta$ = const. rotieren dann starr, aber mit unterschiedlicher Winkelgeschwindigkeit um das Fächerzentrum. Die Verzerrungsgeschwindigkeiten verschwinden offenbar nicht für konstantes, sondern für zum Abstand vom Fächerzentrum proportionales $g(n)$, was sofort die Bedeutung des zweiten Klammerausdruckes (9.48) erhellt.

Im Bereich von Lasteinleitungen zum Beispiel sind auch Spannungs- und Geschwindigkeitsfelder denkbar, deren Fächerzentrum nicht ausserhalb, sondern auf dem Scheibenrand liegt. Entsprechende Geschwindigkeitsfelder zeigt Fig. $9.7 d, e$, wobei sich d) und e) nur um Starrkörperterme unterscheiden. Das Fächerzentrum $Z$ ist Singularitätsstelle sowohl der Geschwindigkeiten als auch der Spannungen. Wegen der zwischen zwei Charakteristiken $\xi$ = const. konstanten Spannungsänderung der $x$-Armierung sind die Verbundspannungen in $Z$ natürlich unendlich. Da diese jedoch in den Fliessbedingungen (2.7) als interne Reaktionen behandelt sind, sind solche Fäl- 


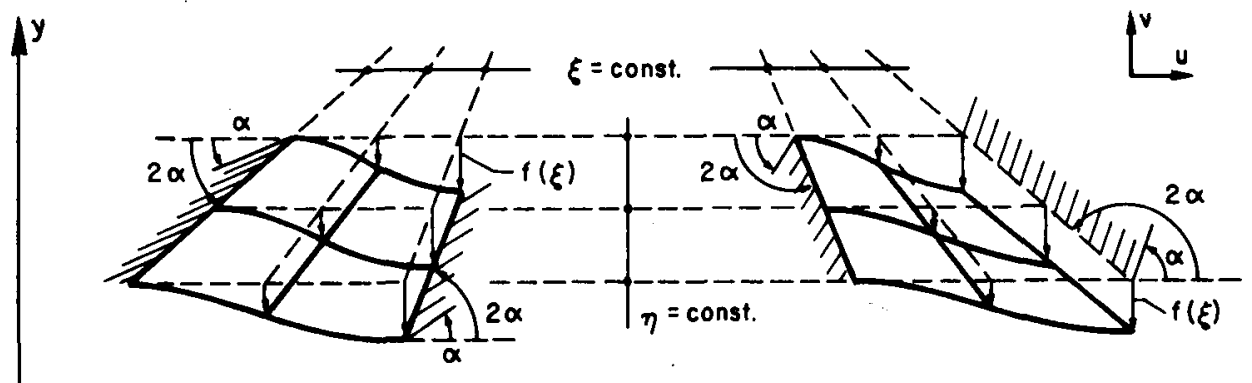

(a) $\dot{\varepsilon}_{y} \geq 0 \quad u=0, v=f(\xi) \quad$ (b) $\varepsilon_{y} \leq 0$

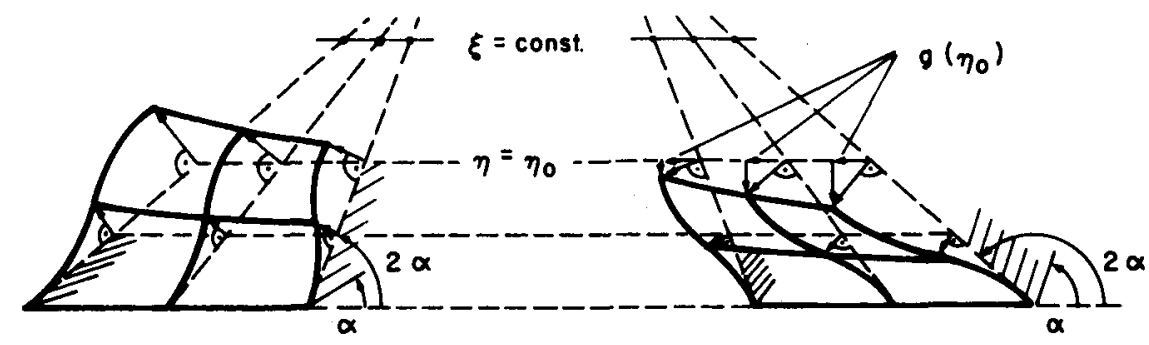

(c) $\dot{\varepsilon}_{y} \geq 0 \quad u=g(\eta), v=-g(\eta) \cot 2 \alpha \quad$ (d) $\dot{\varepsilon}_{y} \leq 0$

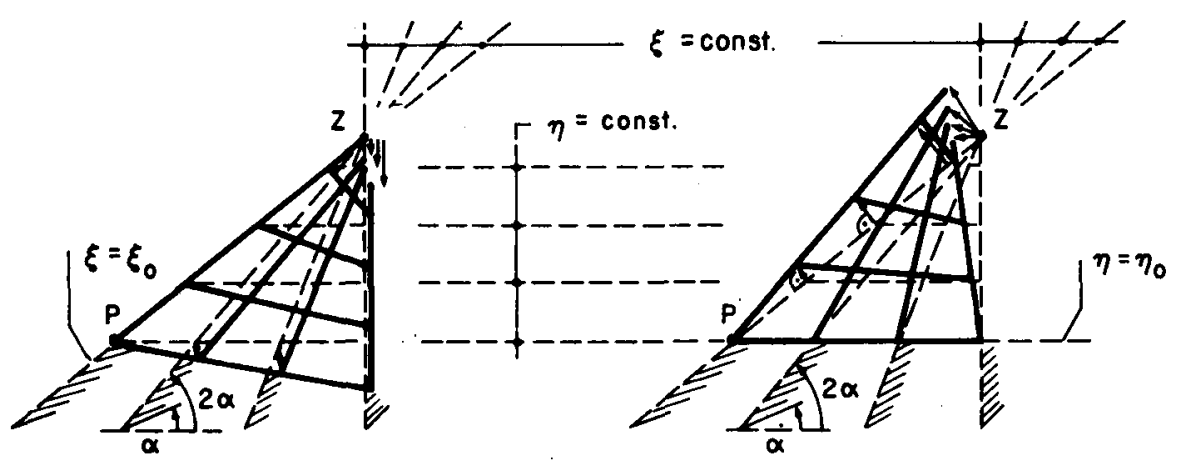

$\begin{array}{ll}\text { (d) } v=-c\left(\xi-\xi_{0}\right) & \text { (e) } u=-c\left(\eta-\eta_{0}\right), v=+c\left(\eta-\eta_{0}\right) \cot 2 \alpha\end{array}$

Fig. 9.7 Geschwindigkeitsfelder in den Regimes III, IN, VII

le in theoretisch exakten Lösungen nicht auszuschliessen. Analoge Felder treten zum Beispiel im Bereich des Stempelrandes beim Stempelproblem des ebenén Dehnungszustandes auf.

\subsection{Ergänzungen}

Noch nicht behandelt worden sind die Geschwindigkeitsfelder, welche mit Spannungszuständen an Kanten oder Ecken der Fliessfigur verträglich sind. In nicht zu einer Linie degenerierten Zonen, die ausschliesslich in einem der Fliessregimes C-D, I-K, G-H, E-F (Fig. 3.2) plastifiziert sind, muss gemäss Abschnitt 9.1 Gleichung (9.26) das stetig vorausgesetzte Spennungsfeld konstant sein. Die Klasse der Geschwindigkeitsfelder, die mit statisch zulässigen Span- 
nungsfeldern in einem dieser Fliessregimes verträglich sein können, muss deshalb - neben den entsprechenden kinematischen Bindungen gemäss Fig. 3.2 - die Bedingung konstanter Richtung der Hauptdehnungsgeschwindigkeiten erfüllen. In den Fliessregimes $A$ und $B$ dagegen (Fig. 3.2, Kegelspitzen) ist natürlich jede Richtung Hauptachsenrichtung des Betonspannungszustandes, und die verträglichen Geschwindigkeitsfelder brauchen nur die kinematischen Bindungen gemäss Fig. 3.2 zu erfüllen.

Aus den allgemeinen Formeln für die in den verschiedenen Fliessregimes möglichen Spannungsund Geschwindigkeitsfelder ist auch sofort ersichtlich, welche Unstetigkeiten diese Felder aufweisen dürfen. Dabei ist jedoch zu beachten, dass die in den vorangehenden Abschnitten abgeleiteten Beziehungen jeweils nur für ein spezielles Fliessregime gelten. Die wichtigen Unstetigkeiten des Spannungsfeldes, bei welchen der Spannungszustand über die Unstetigkeitslinie in ein anderes Fliessregime springt, bedürfen deshalb einer gesonderten Betrachtung.

Bei den Differentialgleichungen (9.12) und (9.38) für die Drucktrajektorienneigung $\alpha$ wurde Stetigkeit der Koeffizienten der Differentialgleichung, d.h. von $\alpha$, vorausgesetzt. Unstetigkeiten in der Betonhauptspannungsrichtung a sind deshalb nur möglich, wenn der Spannungszustand von einem Fliessregime ins andere springt.

Dagegen darf in Gleichung (9.22), die die Betondruckspennung $n_{2}$ für im Fliessregime I plastifizierte Zonen beschreibt, die Funktion $g(\xi)$ beliebig und insbesondere auch unstetig gewählt werden. Die Charakteristiken bzw. Betondrucktrajektorien $\xi=$ const. sind deshalb mögliche Unstetigkeitslinien, über die hinweg die Betonhauptspannung $n_{2}$ springen darf.

Bei der Ableitung der Ausdrücke (9.33) für die Geschwindigkeitsfelder im Regime I plastifizierter Zonen wird in Gleichung (9.30) offensichtlich Stetigkeit der willkürlichen Funktion $f(\xi)$ vorausgesetzt. Dagegen darf $f^{\prime}(\xi)$ und $g(\xi)$ auch unstetig gewählt werden. Die Charakteristiken bzw. Betondrucktrajektorien sind folglich auch mögliche Unstetigkeitslinien des Geschwindigkeitsfeldes. Aus Fig. 9.4 a,b ist sofort ersichtlich, dass Unstetigkeiten von $f^{\prime}$ und $g$ in $\xi=$ a entlang den Geraden $\xi=$ a linear verteilte und zu ihnen senkrechte Geschwindigkeitssprünge zur Folge haben. Dabei sind natürlich nur Sprünge zulëssig, die im Sinne der Konvention (3.14) positiv sind. Solche Unstetigkeiten sind in Abschnitt 3.3 als "Kollapsrisse" bezeichnet worden, und diese sind im Fliessregime I immer Geraden. Ueber einen Kollapsriss hinweg darf natürlich auch die zu ihm tangentiale Betonhauptspannung $\mathrm{n}_{2}$ springen.

In den Fliessregimes III-VII sind die Betonhauptspannungen konstant: $n_{1}=0, n_{2}=-P_{b}$. Da die Betondrucktrajektorienrichtung, wie schon erwähnt, stetig verlaufen muss, sind auch die Betondruckfelder stetig. Dagegen derf in Gleichung (9.44), die die Beanspruchung $z_{x}$ der in den Fliessregimes $V$, VII nicht messgebenden Armierung in x-Richtung beschreibt, $f(n)=f(y)$ auch unstetig gewählt werden. Die Feststellung, dass $z_{x}$ über Unstetigkeitslinien $y=$ const. hinweg springen darf, ist natürlich trivial.

In den Gleichungen (9.47) für die Geschwindigkeitsfelder in Zonen, die in den Regimes $V$, III, VII fliessen, dürfen die beiden willkürlichen Funktionen $f(\xi)$ und $g(n)$ Unstetigkeiten aufweisen. Beide charakteristischen Geradenscharen $\xi=$ const., $n=$ const. sind somit mögliche Unstetigkeitslinien des Geschwindigkeitsfeldes. Aus Fig. 9.7 ist sofort ersichtlich, dass die Sprungrichtung immer senkrecht zu derjenigen charakteristischen Richtung ist, die nicht Unstetigkeitslinie ist. Entlang Unstetigkeitslinien $\xi$ = const. ist der Sprungvektor selbst konstant, entlang Unstetigkeitslinien $n$ = const. im allgemeinen nur seine zur Unstetigkeitslinie tangentiale Komponente. Solche Unstetigkeiten sind in Abschnitt 3.3 als "Gleitlinien" bezeichnet worden, und diese können in den Fliessregimes III-VII nur Geraden sein. 
Gekrümte "Gleitlinien" bzw. "Kollapsrisse" können offenbar nur in plastifizierten Zonen auftreten, wo der Spannungszustand an Kanten bzw. an den Kegelspitzen der Fliessfigur liegt und somit konstant sein muss, wenn die Zone eine endliche Ausdehnung besitzt.

Unstetigkeiten des Spannungsfeldes, bei welchen der Spannungszustand von einem Fliessregime ins andere springt, werden im Rahmen dieser Arbeit nicht in allgemeiner form behandelt. Eine Besonderheit bei Stahlbetonscheiben bedarf jedoch spezieller Erwähnung: Die Unstetigkeiten sind vielfach von unendlich grossen Verbundspannungen begleitet und werden deshalb leicht voreilig ausgeschlossen, wenn man den wirklichen Werkstoff Stahlbeton vor Augen hat.

Als einfaches Beispiel für eine solche Unstetigkeitslinie sei die Neutralachse eines auf Biegung und Schub beanspruchten Balkens betrachtet, die beim Kollaps im Steg liegt. Wird die $x$-Achse in die Neutralachse gelegt, so müssen über die Neutralachse hinweg die Scheibenschnittkräfte $N_{x y}$, $N_{y}$ stetig verlaufen, während $N_{x}$ beim Kollaps springt. Befindet sich der Spannungszustand unterhalb der Neutralachse mit $N_{y}=0, N_{x y}=0.3 P_{b}, N_{x} \approx-0.25 P_{b}$ (Fig. 2.5) im Fliessregime $I$, so liest man aus Fig. 2.5 sofort ab, dass er oberhalb der Neutralachse mit $N_{y}=0, N_{x y}=0.3 P_{b}, N_{x} \approx-0.9 P_{b}$ im Fliessregime VI liegt. An diesem Spannungspunkt liegt aber die Bügelkraft $z_{y}$ rund $40 \%$ unterhalb der Fliesskraft $P_{y}$, während unterhalb der Neutralachse im Fliessregime $I z_{y}=P_{y}$ gilt. Ueber die Neutralachse hinweg verläuft also nur die totale Scheibenschnittkraft $N_{y}$ stetig. Die Schnittkräfte $z_{y}$, $n_{y}$ der Komponenten Stahl und Beton dagegen springen. Lediglich ihre Sprünge heben sich auf. In der Neutralachse treten folglich unendlich grosse Verbundspannungen auf, die sich in einem Knick der Betondrucktrajektorien manifestieren. Diese sind theoretisch zulässig, weil die Verbundspannungen in den Fliessbedingungen (2.7) als interne Reaktionen behandelt sind. Sie dürfen folglich auch nicht ausgeschlossen werden, wenn man theoretisch exakte Lösungen aufgrund dieser Fliessbedingungen sucht. Arbeitet man andererseits mit der wirklichkeitsnaheren Fliessfigur (Fig. 4.4), so verlaufen im gleichen Fall auch die Schnittkräfte $z_{y}=P_{y}$, $n_{y}$ der einzelnen Komponenten Beton und Stahl stetig. Oberhalb der Neutralachse befindet sich der Beton nun in einem zweiaxialen Druckspannungszustand, während die Bügelarmierung an der Zug-, die Längsarmierung an der Druckfliessgrenze liegt.

In Abschnitt $g$ ist nie mit den totalen Scheibenschnittkräften $N_{x}, N_{y}$, $N_{x y}$ gearbeitet worden. Dies liegt natürlich darin begründet, dass die Hauptachsen der Dehnungsgeschwindigkeiten mit jenen des Betonspannungszustandes zusammenfallen und nicht mit denjenigen von $N_{x}, N_{y}, N_{x y}$. Die Fliessfigur in $N_{x}, N_{y}, N_{x y}$ erleichtert jedoch die Diskussion der massgebenden Fälle wesentlich. Sie bringt auch zum Ausdruck, dass man - wie obiges Beispiel und das folgende zeigen - mit einem fiktiven theoretischen Material arbeitet: Während es im Fliessregime I (und II) keine Rolle spielt, ob die Armierung verschmiert oder diskret verteilt ist, weil keine Verbundspannungen übertragen werden, sind die Beziehungen für die übrigen Fliessregimes nur für unendlich fein verschmierte Armierung gültig.

Schliesslich sei auch noch auf den Zusammenhang zwischen Scheiben- und Plattentheorie hingewiesen: Die in der Plastizitätstheorie der Stahlbetonplatte üblicherweise verwendete Fliessfigur [31] entspricht der Fliessfigur einer Sandwichplatte, die aus zwei der Fliessbedingung (2.7 I) gehorchenden Scheiben besteht. Im Fliessregime I der Scheibe ist die Spannungsfunktion des Betons eine abwickelbare Fläche, bei der Platte die Durchbiegung, solange nur eine der Sandwichscheiben fliesst. Bei beiden bilden unter der gleichen Voraussetzung die Hauptrichtungen der Verzerrungs- bzw. Krümmungsgeschwindigkeiten im allgemeinsten Fall unzentrierte Fächer, und dem Begriff der Fliessgelenklinie entspricht derjenige des Kollapsrisses. 
Der Bemessung der Schubarmierung werden gemäss [29, 30] Stegspannungszustände im Regime I zugrunde gelegt. Die in Abschnitt 9.1 hergeleiteten Beziehungen erlauben es nun, auch bei variabler Querkraft, Bügelarmierung oder Balkenhöhe statisch zulässige Spannungsfelder für die im Regime I plastifizierte Stegscheibe zu bestimmen. Damit wird das Studium der schon früher aufgeworfenen und für die Praxis relevanten Frage ermöglicht: Garantieren die querschnittsweise Bemessung der Schubarmierung aufgrund der Balkentheorie (Fachwerkmodell, Fig. 6.2 a) und die Kantralle der Betondruckspannungen im Steg über die nominelle Schubspannung die Existenz stabiler, statisch zulässiger Spannungszustände im Steg, wenn die zur Dimensionierung verwendete Diagonalenneigung a gewechselt wird, die Querkraft variiert, Vouten vorhanden sind, oder wenn konzentrierte Einzellasten und Auflagerreaktionen eingeleitet werden?

\subsection{Träger unter verteilter Belastung}

Ein I-, T- oder symmetrischer Kastenträger konstanter Höhe sei durch eine zumindest stückweise konstante, über den obern oder untern flansch aufgebrachte, verteilte Belastung $P=P_{0}+P_{u}$ beansprucht (Fig. 10.1 a). Die Stegarmierung bestehe aus Eügeln mit ebenfalls stückweise konstanter Fliesskraft pro Längeneinheit $P_{y}$. Als im konkreten Fall zu überprüfende Arbeitshypothese wird angenommen, dass im betrachteten Stegbereich unter der Bemessungsbruchlast die Bügel fliessen und die Betondruckspannungen unterhalb der Druckfestigkeit liegen. Die Stegscheibe ist somit im Regime I plastifiziert und die Betondruckspannungen sind durch (9.22) gegeben.

An den Stegrändern $\eta_{U}=0, n_{0}=h$ sind lediglich Randbedingungen in den Normalspannungen $N_{y}$ $z u$ erfüllen. Die Schubspannungen $N_{x y}$ werden durch die Flanschen bzw. Hauptlängsarmierung aufgenommen. Die Randbedingungen lauten mit (2.1) (Fig. 10.1 a)

$\left.\begin{array}{l}N_{y}\left(n_{0}\right)=n_{y}^{0}+P_{y}=-p_{0}, \\ N_{y}\left(n_{u}\right)=n_{y}^{u}+P_{y}=+p_{u} .\end{array}\right\}$

Aus (10.1) und der Beziehung (9.22) für die Betonspannungen gewinnt man sofort die Differentialgleichung für das Drucktrajektorienfeld $k(\xi)$ in einem Bereich konstanter Last und Bügelarmierung.

$\frac{-n_{y}^{u}}{-n_{y}^{0}}=\frac{P_{y}-p_{u}}{P_{y}+P_{0}}=\frac{n_{2}^{u} \sin ^{2} \alpha(\xi)}{n_{2}^{0} \sin ^{2} \alpha(\xi)}=\frac{1+n_{0} k^{\prime}(\xi)}{1+n_{u} k^{\prime}(\xi)}$.

Mit $\eta_{u}=0, \eta_{0}=h$ ergibt sich

$k^{\prime}(\xi)=-\frac{1}{h} \frac{p_{u}+p_{0}}{P_{y}+p_{0}}$,

$\cot \alpha(\xi)=k(\xi)=\cot \alpha_{u}-\frac{P_{u}+P_{0}}{P_{y}+P_{0}} \frac{\xi}{h}$,

oder

$\cot \alpha(\xi)=k(\xi)=\cot \alpha_{u}-\frac{p}{\left(P_{y}-P_{u}\right)+p} \frac{\xi}{h}$. 


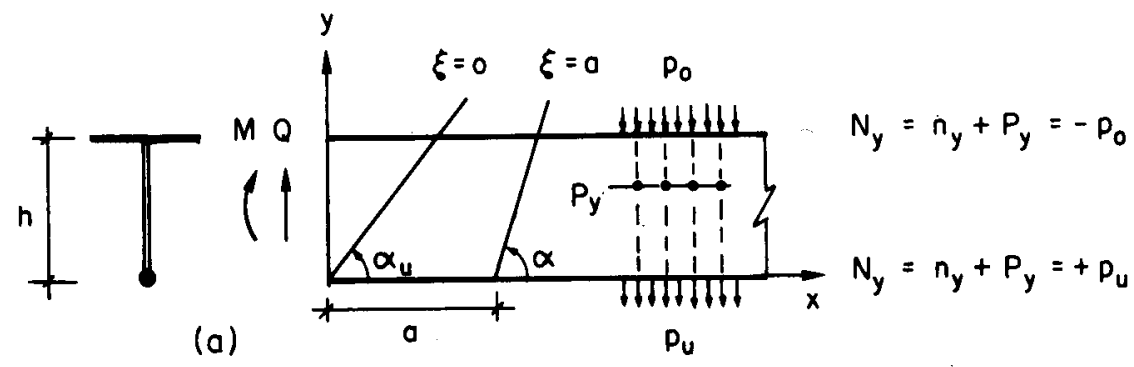

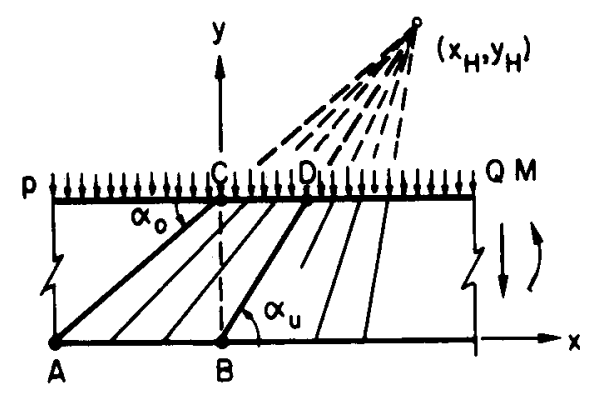

(b)

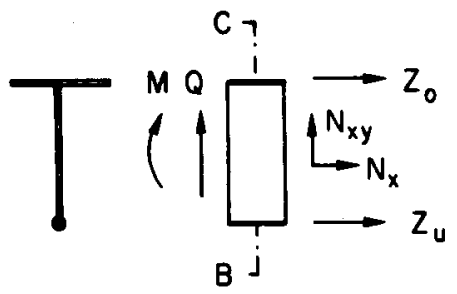

(c)

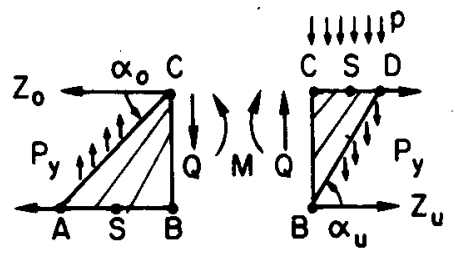

(d)

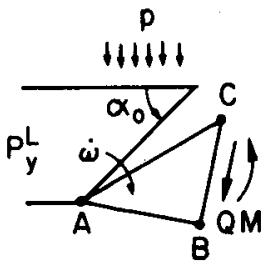

(e)

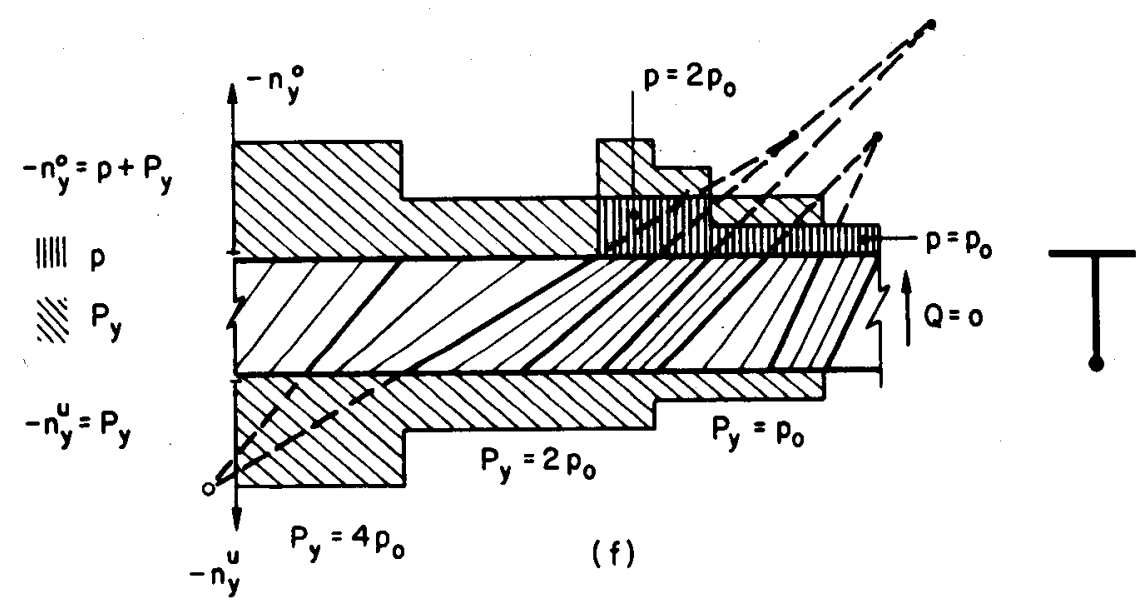

Fig. 10.1 Allgemeine Stegspannungszustände im Regime I für stückweise konstante Lost und Bügelfliesskraft 
Dabei wurde als Integrationskonstante die Drucktrajektorienneigung $\alpha_{u}$ am unteren Rand des Querschnittes $x=0$ eingeführt, $\cot \alpha(\xi=0)=\cot \alpha_{u}$.

Aus (10.1), (10.4), (10.5) ist sofort ersichtlich, dass unten angehängte Lasten $p_{u}$ und eine um eine Aufhängebewehrung $\Delta P_{y}=p_{u}$ verstärkte Bügelarmierung zu den gleichen Betonspannungszuständen im Steg führen wie oben aufgelegte Lasten $p_{0}$ und unverstärkte Bügelarmierung. Ohne Verlust von Allgemeinheit kann man sich deshalb im folgenden auf aufgelegte Lasten beschränken. Angehängte Lasten sind bei der Bemessung durch eine Aufhängebewehrung und bei InteraktionsformeIn durch Ersetzen von $P_{y}$ durch ( $P_{y}-P_{u}$ ) zu berücksichtigen. Dabei wird offensichtlich für angehängte Lasten nur dann die gleiche Schubbruchsicherheit erreicht wie für aufgelegte, wenn die Aufhängebewehrung für die ganze angehängte Last dimensioniert und wie normale Bügel im Druckflansch verankert wird.

Da die Bügelarmierung nur stückweise konstant vorausgesetzt wurde, können oberes und unteres Trajektorienende in Bereichen verschiedener Bügelfliesskraft liegen. Beträgt die Bügelfliesskraft am obern Drucktrajektorienende $P_{y}^{0}$, am unteren $P_{y}^{u}$, so ergibt sich

$\cot \alpha(\xi)=k(\xi)=c-\frac{\left(P_{y}^{0}-P_{y}^{u}\right)+P}{P_{y}^{0}+P} \frac{\xi}{h}$

Da $k(\xi)$ linear variiert, $k^{\prime}(\xi)$ = const., degeneriert die Hüllkurve (9.8) zu einem Punkt $x_{H}$ $\mathrm{y}_{H^{*}}$ Die Drucktrajektorienfelder (10.4) bis (10.6) stellen zentrierte Fächer dar, die sich auch in der Form

$k(\xi)=\cot \alpha(\xi)=\left(x_{H}-\xi\right) / y_{H}$

schreiben lassen (Fig. 10.1 b).

Die Beziehung zwischen den Drucktrajektorienneigungen $\alpha_{u}(B)$ und $\alpha_{0}$ (C) am untern bzw. obern Stegrand im Querschnitt $x=0$ ist mit (10.5) für $P_{y}=$ const., $P_{u}=0$ gegeben durch

$\cot \alpha_{0}=\cot \alpha_{u} \frac{P_{y}+P}{P_{y}}$,

wenn $\xi(C)=0-h \cot \alpha_{0}$ beachtet wird (Fig. 10.1 b). (10.8) drückt natürlich lediglich das vertikale Gleichgewicht des Betonstreifens ABCD aus. Die Strecken AB, CD stehen im umgekehrten Verhältnis zu ihren "Lasten" $P_{y} b z w . P_{y}+P$.

Für stückweise konstante verteilte Belastung und Bügelfliesskraft setzt sich das Betondrucktrajektorienfeld im plastifizierten Steg also aus einer Folge von zentrierten Fächern und Bereichen konstanter Trajektorienneigung zusammen. Ausgehend von einer Stelle bekannter Querkraft kann man das Trajektorienfeld sofort streifenweise, von Sprungstelle zu Sprungstelie der "Lasten" $P_{y}$ und $p$ fortschreitend, gewinnen (Fig. $10.1 \mathrm{f}$ ).

Bei Vouten geht in die vertikale Gleichgewichtsbedingung finiter Betonstreifen zwischen zwei Drucktrajektorien auch die Zugkraftvariation der Längsarmierung ein. Das Trajektorienfeld lässt sich in diesem Fall einfacher direkt über die Differentialgleichung gewinnen (Abschnitt 10.2$)$.

Da die maximalen Betonspannungen im Steg immer an den Flanschen auftreten, lassen sich diese sofort aus den Randbedingungen bestimmen, sobald das Trajektorienfeld bekannt ist. Der Zugkraftverlauf in der Hauptlängsarmierung folgt ebenfalls sofort aus der Integration der Schub- 
spannungen $N_{x y}=n_{x y}\left(\eta_{u, 0}\right)$, wenn die Zugkraft in einem Querschnitt bekannt ist.

Die Gleichgewichtsbedingungen für den Querschnitt B-C bzw. $x=0$ (Fig. 10.1 b,c) lauten

$\left.\begin{array}{rl}Q=-\int_{0}^{h} N_{x y} d y=-\int_{0}^{h} n_{2} \sin \alpha \cos \alpha d y, \\ z_{u}+z_{0}=-\int_{0}^{h} N_{x} d y=-\int_{0}^{h} n_{2} \cos ^{2} \alpha d y, \\ z_{0} h+M=-\int_{0}^{h} N_{x} y d y=-\int_{0}^{h} n_{2} \cos ^{2} \alpha y d y,\end{array}\right\}$

wobei a als Funktion von $\xi(x=0, y)$ aufzufassen ist. Wird vorerst angenommen, dass $p$ und $P_{y}$ gemäss Fig. 10.1 b im Bereich ABCD konstant sind, so ist $n_{2}$ mit $(9.22),(10.1),(9.9)$ und (9.17) gegeben durch

$n_{2}=\frac{g(\xi)}{1+n k^{\prime}(\xi)}=-\frac{P_{y} / \sin ^{2} \alpha(\xi)}{1+n k^{\prime}(\xi)}=\frac{P_{y}}{\sin \alpha \cos \alpha}, y$

Beachtet man, dass $\xi_{B}=\xi(x=0, y=0)=0, \xi_{C}=\xi(x=0, y=h)=-h$ cota, ist, so folgt durch Einsetzen von $(10.10)$ in $(10.9)$

Q $\quad=-P_{y} \int_{0}^{h} \xi, y=-P_{y} \int_{\xi_{B}}^{\xi_{C}} d \xi=P_{y} h \cot \alpha_{\square}$.

$z_{0} h+M=-P_{y} \int_{0}^{h} y \cot \alpha \xi, y d y=+P_{y} \int_{\xi_{B}}^{\xi_{C}} \xi d \xi=\frac{1}{2} P_{y} h^{2} \cot ^{2} \alpha_{0}=\frac{1}{2} Q \cot \alpha_{0} h$.

$z_{0}+z_{u}=-P_{y} \int_{0}^{h} \cot \alpha \xi, y d y=-P_{y} \int_{\xi_{B}}^{\xi_{C}} \cot \alpha(\xi) d \xi$.

Einsetzen von (10.5) für cota und Beachtung von (10.8) ergibt

$z_{u}+z_{0}=-P_{y} \int_{\xi_{B}}^{\xi_{C}}\left(\cot \alpha_{u}-\frac{P}{P_{y}+P} \frac{\xi}{h}\right) d \xi=\frac{1}{2}\left(\cot \alpha_{u}+\cot \alpha_{0}\right) \cot \alpha_{D} P_{y} h=\frac{1}{2}\left(\cot \alpha_{u}+\cot \alpha_{0}\right) Q=Q \cot \alpha_{m}$.

Die Beziehungen zwischen den Schnittkräften, der Belastung, der Bügelfliesskraft und den Flanschkräften lauten also

$Q=P_{y} h \cot \alpha_{0}=\left(P_{y}+p\right) h \cot \alpha_{u}$,

$(10.11)$

$z_{u}=\frac{M}{h}+\frac{1}{2} Q \cot \alpha_{u}$

$z_{0}=-\frac{M}{h}+\frac{1}{2} Q \cot \alpha_{0}$

Sie hätten natürlich auch sofort aus dem vertikalen Gleichgewicht und der Momentenbedingung um den Schwerpunkt $S$ der "Lasten" $P_{y}$ bzw. $P_{y}+p$ für die Betondreiecke gewannen werden können, die vom betrachteten Querschnitt und den Drucktrajektorien $\alpha=\alpha_{u}$, $\alpha=\alpha_{0}$ begrenzt werden (Fig. $10.1 \mathrm{~d}$ ). Dabei ist jedoch zu beachten, dass die Gleichgewichtsbetrachtung gemäss Fig. 10.1 dallein mit der Mechanismusmethode gemäss Fig. 10.1 e identisch ist, weil damit noch keine stabilen, statisch zulässigen Betonspannungszustände im Bereich ABCD nachgewiesen werden. 
Für $p=0, \alpha_{u}=\alpha_{0}=\alpha$ gehen die Gleichungen (10.11), (10.12) in die Beziehungen des Fachwerkmodelles bzw. der Balkentheorie gemäss Abschnitt 6.3.2 über. Die Voraussetzung eines im betrachteten Querschnitt konstanten Stegspannungszustandes steht also für die Annahme, dass einerseits $P$ gegenüber $P_{y}$ vernachlässigbar sei, und dass andererseits die Bügelarmierung über die Länge der vom oberen und unteren Querschnittsrand ausgehenden Drucktrajektorien konstant sei. Letzteres ist insbesondere bei der Dimensionierung zu beachten.

Aus Fig. 10.1 d ist sofort ersichtlich, dass in den Gleichungen (10.12) für nicht konstantes $P_{y}$ bzw. $p$ nur der Faktor $1 / 2$ ändert, weil der Schwerpunkt $S$ nicht mehr in der Mitte der strecken CD bzw. AB zu liegen braucht, während in (10.11) nun Integrale über diese Strecken auftreten. Insbesondere ändern sich die Beziehungen für $Z_{u}$, $Z_{0}$ überhaupt nicht, wenn Bügelfliesskraft $P_{y}$ und Last $p$ nur rechts und links des Querschnittes konstant sind, im Querschnitt selbst aber springen. Das Trajektorienfeld ist mit (10.6) immer noch ein zentrierter Fächer, und die Bestimmungsgleichung (10.11) für cot $\alpha_{u}$, cota kann sofort verallgemeinert werden auf

$Q=P_{y}^{L} h \cot \alpha_{0}=\left(P_{y}+P\right)^{R} h \cot \alpha_{u}$.

Setzt man für $Z_{u}$, $Z_{\text {o }}$ die Fliesskräfte $Z_{u f}$, $Z_{\text {of }}$ ein und eliminiert cot $\alpha_{u}$, cota, so kann man (10.12) und (10.13) auch in der Form einer verallgemeinerten, die Normalspannungen $N_{y}$ quer zur Balkenaxe berücksichtigenden M-Q-p-Interaktionsbeziehung schreiben

$$
\begin{aligned}
& \frac{M}{h}+\frac{Q^{2}}{2\left(P_{y}+p\right)^{R} h}=z_{u f}, \\
& -\frac{M}{h}+\frac{Q}{2 P_{y}^{L} h}=z_{\text {of }} .
\end{aligned}
$$

Da die zusätzlichen Zugkräfte $z_{u}$, $Z_{0}$ infolge Querkraft nur von der Drucktrajektorienneigung $\alpha_{u}$ am untern bzw. $\alpha_{0}$ am obern Stegrand des Querschnittes abhängen und damit nur von der Bügelfliesskraft auf jeweils einer Querschnittsseite beeinflusst werden, können die zugehörigen verträglichen Mechanismen nur die in Fig. 10.1 e dargesteliten, schon in Abschnitt 6.4 eingeführten Kollapsrissmechanismen sein. Mit ihnen folgt (10.14) auch unmittelbar über die Mechanismusmethode.

(10.14) stellt die exakte Interaktionsbeziehung für einen Querschnitt mit links bzw. rechts vom Querschnitt konstanter Bügelfliesskraft und verteilter Belastung dar, wenn die Mechanismen Fig. 10.1 e kinematisch zulässig sind, und die Betondruckfestigkeit von den Betonspannungen nicht überschritten wird. Diese betragen in den Punkten A und C links (die Punkte B,C sind als Sprungstellen der Bügelfliesskraft auch solche der Betonspannung)

$$
\begin{aligned}
& n_{2}=\sigma_{b} b_{0}=-P_{y}^{L} / \sin ^{2} \alpha_{0}=-P_{y}^{L}\left(1+\cot ^{2} \alpha_{0}\right), \\
& \sigma_{b}=-\frac{Q}{b_{0} h} \tan \alpha_{0}\left(1+\cot ^{2} \alpha_{0}\right)=-\frac{Q}{b_{0} h} \frac{1}{\sin \alpha_{0} \cos \alpha_{0}},
\end{aligned}
$$

und entsprechen in den Punkten $D$ und $B$ rechts

$$
\sigma_{b}=-\frac{Q}{b_{0} h} \tan \alpha_{u}\left(1+\cot ^{2} \alpha_{u}\right)=-\frac{Q}{b_{0} h} \frac{1}{\sin \alpha_{u} \cos \alpha_{u}} \text {. }
$$

Die Spannungen in diesen Punkten sind offensichtlich mit jenen identisch, welche unter der Annahme eines über den Querschnitt konstanten Betonspannungszustandes mit Trajektoriennei- 
gung $\alpha_{0}$ bzw. $\alpha_{u}$ errechnet werden. Die maximalen Betonspannungen können wesentlich häher liegen und treten in jenen Punkten auf, wo die flachere Drucktrajektorie auf die stärkere Bügelfliesskraft trifft, d.h. für $\left(P_{y}+p\right)^{R} \geq P_{y}^{L}$ im Punkt $C$ rechts:

$n_{2}=-\left(P_{y}+p\right)^{R} / \sin ^{2} \alpha_{0}=-\left(P_{y}+P\right)^{R}\left(1+\cot ^{2} \alpha_{0}\right)$

$\sigma_{b}=-\frac{Q}{b_{0} h} \tan \alpha_{u}\left(1+\cot ^{2} \alpha_{0}\right)$,

und für $\left(P_{y}+P\right)^{R} \leq P_{y}^{L}$ im Punkt B links:

$\sigma_{b}=-\frac{Q}{b_{0} h} \tan \alpha_{0}\left(1+\cot ^{2} \alpha_{u}\right)$.

Im folgenden sollen aus den Ergebnissen einige Schlüsse gezogen werden.

In verschiedenen Schubversuchen sind die Versuchsbalken ausserhalb eines kürzeren eigentlichen Testbereichs durch zusätzliche Längs- und Schubarmierung verstärkt worden zwecks eindeutiger Lokalisierung von Schubbruch und Messbereich. Stellt die Zone links von BC in Fig. 10.1 b den mit konstanter Längs- und Schubbewehrung armierten Testbereich dar, während rechts von BC Längs- und Schubarmierung verdoppelt sind, so liegt der massgebende Querschnitt an der Grenze BC. Theoretisch kann dann mit dem Fliessen der Bügel links und der Längsarmierung in $B$ die Traglest noch nicht erreicht sein. Ein Mechanismus im Regime I ist gemäss (10.14) erst möglich, wenn auch die Bügel rechts fliessen und sich der Kollapsriss BD öffnen kann (Fig. 10.1 e). Da dann die Betonspannungen im Punkt C gemäss (10.16) doppelt so hoch sind als im Testbereich vorgesehen; dürfte der Spannungszustand kaum mehr stabil sein. Eine exakte Lösung für die Traglast unter Einbezug der Fliessregimes mit massgebender Betondruckfestigkeit ist nicht nur für die Interpretation der Versuchsresultate, sondern auch theoretisch interessant, da davon Anhaltspunkte für die Anwendbarkeit der Plastizitätstheorie zu erwarten sind. Grundlagen dazu sind in Abschnitt $g$ zusammengestellt.

Von grösserer praktischer Bedeutung ist die Frage, unter welchen Voraussetzungen die querschnittsweise Bemessung aufgrund der Querschnittsinteraktionsbeziehungen (6.15) der Balkentheorie (Fachwerkmodel1) die Existenz stabiler statisch zulässiger Scheibenspannungszustände im Steg gewährleistet. Gemäss Richtlinie 34 [29] ist der Bemessung der Schubarmierung ein "Fachwerkmodell mit variabler Diagonalenneigung $3 / 5 \leq$ tana $\leq 5 / 3$ " (Fig. 6.2 a) zugrunde zu legen, während die minimalen Betonabmessungen des Steges aus der Begrenzung der nominellen Schubspannungen folgen. Die Bestimmung der Armierungskräfte $z_{y}, z_{u}, z_{0}$ gemäss Fig. 6.2 a kann damit offenbar in verschiedenen Teilbereichen eines Balkens aufgrund verschiedener Diagonalenneigungen $\alpha$ erfolgen.

Nun stellen jedoch (10.14) und damit auch die darin enthaltene Fachwerkmodellinteraktion im strengen Sinne keine Querschnittsinteraktion, sondern eine Interaktionsbeziehung für die Trägerzone ABCD dar (Fig. 10.1 b,d,e). Benachbarte Querschnitte können deshalb im Prinzip nicht unabhängig voneinander dimensioniert werden: Für die - wie später noch deutlicher wird - ungünstigste Beanspruchung durch eine konstante Querkraft ist die Bestimmung von $Z_{u}$ ' $Z_{0}$ im betrachteten Querschnitt mit dem Fachwerkmodell (Fig. 6.2 a) nur dann auf der sicheren Seite, wenn die für diesen Querschnitt erforderliche Bügelfliesskraft mindestens über $h \cdot \cot \alpha$ über den Querschnitt hinaus vorhanden ist. Wird bei querschnittsweiser Bemessung die Diagonalenneigung a gewechselt, braucht dies nicht mehr der Fall zu sein. Kritisch können aber insbesondere die mit einem Wechsel von a verbundenen Spannungskonzentrationen im Stegbeton werden. Ist in einem Querschnitt mit tan $\alpha_{\text {dim }}$ dimensioniert worden, $P_{y}=(Q / h)$ tana dim während die Berechnung des Trajektorienfeldes nach der Scheibentheorie am obern oder untern 
Querschnittsrand $\tan \alpha_{\text {vorh }}$ ergibt, so beträgt dort die Betonspannung, wenn $p$ gegenüber $P_{y}$ vernachlässigt wird

$\sigma_{b} b_{0}=n_{2}=-P_{y} / \sin ^{2} \alpha_{v o r h}$,
$\sigma_{b}=-\frac{Q}{b_{o} h} \tan \alpha_{d i m}\left(1+\cot ^{2} \alpha_{v o r h}\right)$.

Obschon auch tan $\alpha_{\text {vorh }}$ innerhalb der in der Norm vorgeschriebenen Grenzen liegen muss, kann die Betonspannung gemäss (10.17) für tan $\alpha_{v o r h}<\tan \alpha_{\text {dim }}$ wesentlich über jenen Werten liegen, die sich aufgrund eines konstanten Spannungszustandes im Steg mit tana ${ }_{\text {vorh }}=\operatorname{tana}_{\text {dim }}$ errechnen. Dies sei anhand eines bewusst extrem gewählten Beispiels veranschaulicht.

Fig. 10.2 a zeigt einen Träger unter Biegung und konstanter Querkraft, bei dem eine erste, mit $\tan \alpha_{d i m}=3 / 5$ dimensionierte zone von einer zweiten, mit tan $\alpha_{d i m}=5 / 3$ bemessenen gefolgt wird. Die bei querschnittsweiser Bemessung nach Fachwerkmodell erforderlichen Bügel- und Längsarmierungsfliesskräfte infalge Querkraft sind strichpunktiert eingetragen. Ist die tatsächliche Armierung der erforderlichen exakt angepasst so fliesst unter der Bemessungsbruchlast die gesamte Armierung, und das Betondrucktrajektorienfeld kann im Bereich der Sprungstelle der Bügelfliesskraft mit (10.6) nur der eingezeichnete zentrische Fächer sein. Die zugehörigen Flanschkräfte infolge Querkraft sind ausgezogen bzw. strichliert eingetragen. Dabei lassen sich in den Punkten A,B,C,D direkt die Formeln (10.12) anwenden, während sich der Verlauf dazwischen aus den Schubspannungen bestimmt.
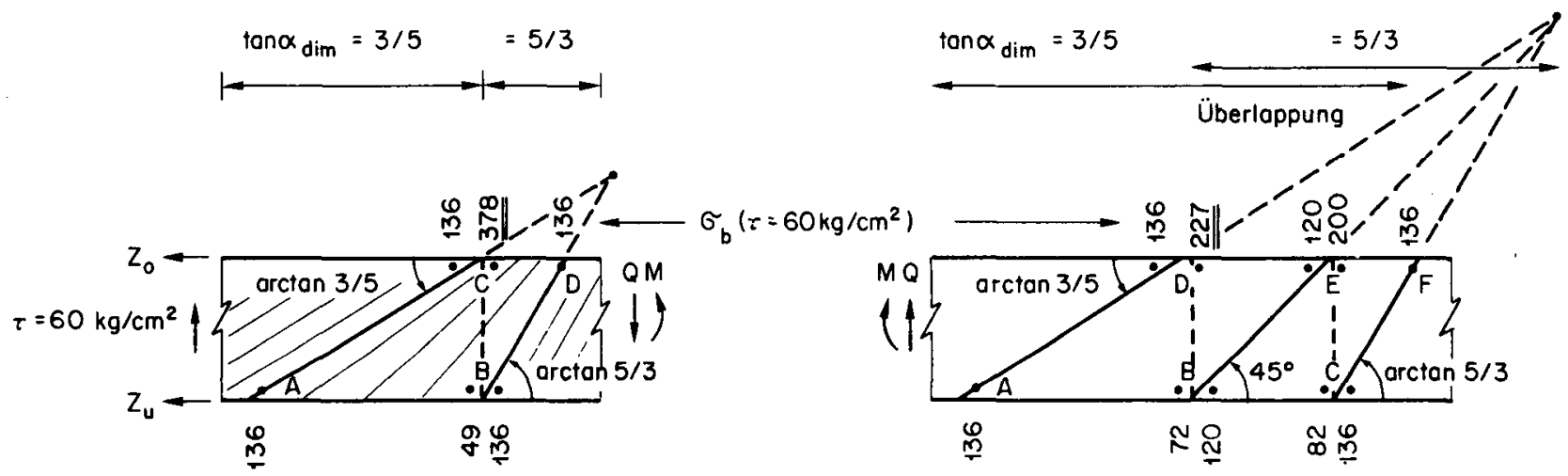

(a)
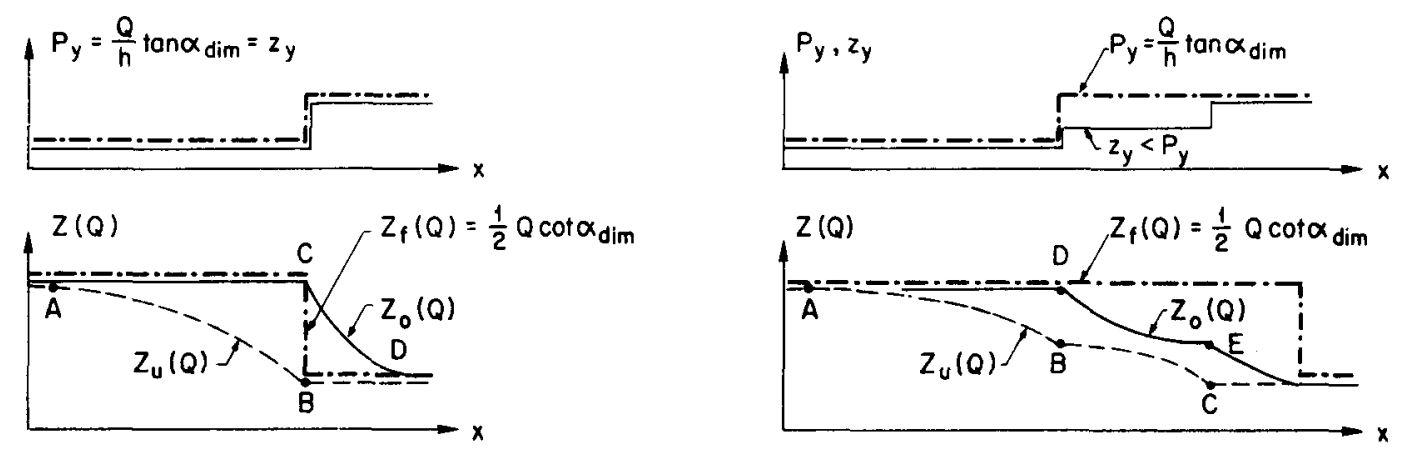

-.-.- vorhandene bzw. erforderliche Fliesskruffe gemóss Bolkentheorie / Fochwerkmodell

- entsprechende Armierungskräfte gemöss Scheibentheorie

Fig. 10.2 Vergleich Bolkentheorie/Scheibentheorie 
Ob die obere Flanschzugkraft $Z_{0}$ für ein negatives Moment stabil ist, hängt von der Vorbinde- und Verankerungslänge der Armierung ab. Kritisch sind jedoch eindeutig die Betonspannungen. Ist die obere Schubspannungsgrenze gemäss Richtlinie 34 [29] ausgenützt, $Q / b_{0} h=$ $60 \mathrm{~kg} / \mathrm{cm}^{2}\left(\mathrm{BH} 300, \beta_{p}=225 \mathrm{~kg} / \mathrm{cm}^{2}\right)$, so beträgt die Betondruckspannung im Punkt $\mathrm{C}$ rechts mit (10.16a) oder $(10.17)$

$\sigma_{b}=-60 \frac{5}{3}\left(1+\left(\frac{5}{3}\right)^{2}\right) \sim-380 \mathrm{~kg} / \mathrm{cm}^{2}::$

Konstruktiv ungeschickt ist natürlich nicht die Verstärkung der Bügelarmierung rechts, sondern die zu schwache Längsarmierung im Querschnitt BC, die keine flachere Neigung der Trajektorie BD zulässt. Eine Ueberlappung der Armierungssprungstellen baut die Spannungskonzentration schon erheblich ab, wie Fig. 10.2 b anhand eines möglichen Spannungszustandes zeigt, der sicher noch nicht optimal ist.

Das Beispiel zeigt, dass eine einmal gewählte Diagonalenneigung $3 / 5 \leq$ tana dim $\leq 5 / 3$ bei voll ausgenützter oberer Schubspannungsgrenze in Längsrichtung konstant anzuwenden ist. In Bereichen niederer Schubbeanspruchung sind bei einem Wechsel die Armierungssprungstellen mind. um h zu überlappen. Wird die querschnittsweise Bemessung der Schubarmierung mit in Längsrichtung konstantem tan $\alpha_{\text {dim }}$ durchgeführt, so lassen sich auch für variierende Querkraft praktisch immer stabile statisch zulässige Spannungszustände in Flanschen und Stegscheibe finden. Dabei werden die Betonspannungen im Steg dann als stabil betrachtet, wenn sie diejenigen Werte nicht überschreiten, die sich für den Ansatz eines über den Steg konstanten Betonspannungszustandes ergeben. Diese Werte sind durch die Begrenzung der nominellen Schubspannung und von tana dim bei der Bemessung kontrolliert. Für die Verhältnisse im Bereich von konzentrierten Einzellasten und Auflagern sei auf Abschnitt 10.3 verwiesen.

Am anschaulichsten ist der Vergleich von Balken- und Scheibentheorie natürlich für Lastfälle, für welche Betonspannungszustände im Steg existieren, die wenigstens in isolierten Querschnitten konstant sind. Da im Regime I die Konstanz des Betonspannungszustandes, zwischen BC die Konstanz in ganz ABCD bedingt (Fig. 10.1 b), kommen nur Spannungszustände gemäss Fig. 10.3 a in Frage. Sie können immer als diskrete Fachwerke mit bandartigen "Bügeln" und "Druckdiagonalen" der Breite $h \cdot \cot \alpha_{d i m}$ unter stückweise über $h \cdot \cot \alpha_{d i m}$ konstanter Belastung $p$ aufgefasst werden.

Der Vergleich dieser fachwerkartigen Spannungszustände mit den bei querschnittsweiser Dimensionierung vorhandenen Armierungsfliesskräften und Stegbetonabmessungen (Fig. 10.3 a) zeigt anschaulich, dass für variierende Querkraft bei den Bügeln Reserven vorhanden sind, die ein Steilerstellen der Drucktrajektorien erlauben. Dies entlastet aber die Längsarmierung und den Stegbeton, bei welchen offensichtlich keine Reserven vorhanden sind. Aus diesem Grund sind auch die Auswirkungen eines Wechsels der Diagonalenneigung $\alpha_{\text {dim }}$ für konstante Querkraft am ungünstigsten.

Die Vermutung liegt nahe, dass die volle Ausnützung der bei querschnittsweiser Dimensionierung vorhandenen Bügelfliesskraft immer auf stabile, statisch zulässige Spannungszustände führt. Dies wird im folgenden für einen einfachen Fall verifiziert. Der Kragträger von Fig. 10.3 b sei durch eine beliebige, aber nur am obern Flansch angreifende verteilte Belastung $p(x)$ beansprucht. Für eine bestimmte Trajektorie $\alpha$ = const. folgt aus den Randbedingungen am oberen und unteren Flansch

$\frac{n_{2}^{0}}{n_{2}^{u}}=1-h k^{\prime}(x)=\frac{p(x)+P_{y}(x)}{P_{y}(x-h k(x))}$. 
wobei $\xi$, $\eta$ schon durch $x, y$ ausgedrückt sind, und $k(x)=\cot \alpha(x)$ die Trajektorienneigung auf Höhe des oberen Flansches bezeichnet. Die Integration von (10.18) ergibt natürlich

$$
Q(x)=\int_{x-h k(x)}^{x} P_{y}(\bar{x}) d \bar{x} .
$$

Die querschnittsweise, mit tan ${ }_{d i m}$ dimensionierte Bügelfliesskraft beträgt

$P_{y}(x)=\frac{1}{h} Q(x) \tan \alpha_{d i m}$

(a)
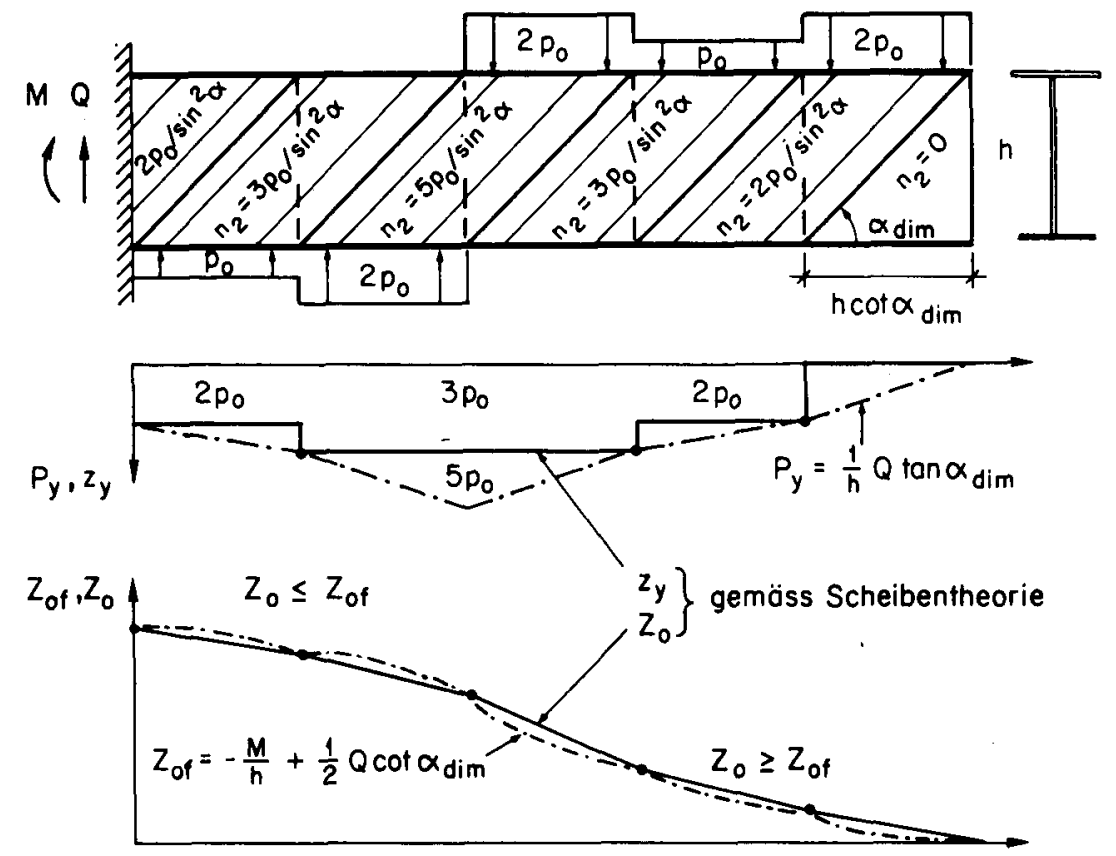

-.-- erforderliche Arm. fliesskraft gemidss Balkentheorie/Fachwerkmodell

(b)

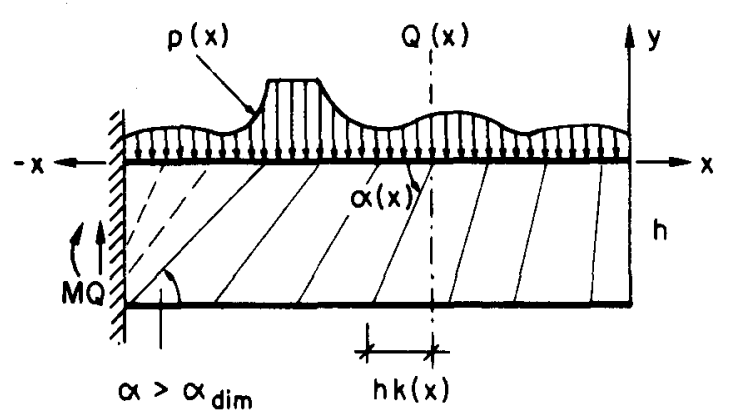

(c)
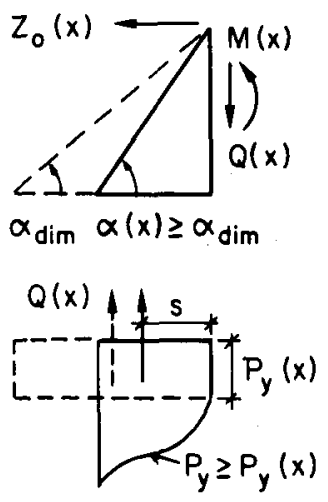

$Z_{0} h=-M+Q \cdot s ; s \leq \frac{1}{2} h \cot \alpha_{\text {dim }}$

Fig. 10.3 Vergleich Balkentheorie/Scheibentheorie 
bewehrung $\Delta P_{y}(x)=|p(x)|$ zu berücksichtigen wäre. Einsatzen von (10.20) in (10.19) ergibt

$$
\left.\begin{array}{c}
Q(x)=\frac{1}{h}\{M(x)-M(x-h k(x))\} \tan \alpha_{d i m}, \\
M(x-h k(x))=M(x)-h \cot \alpha_{d i m} M^{\prime}(x), \\
M^{\prime \prime}=-p \leq 0 .
\end{array}\right\}
$$

(10.21) stellt für gegebenes $M(x)$ eine endliche Bestimmungsgleichung für das Trajektorienfeld $\cot \alpha=k(x)$ dar. Man verifiziert leicht - z.B. für konstantes $p>0-$, dass a vom Wert $\alpha=90^{\circ}$ am freien Ende asymptotisch für $x \rightarrow-\infty$ den Wert $\alpha_{\text {dim }}$ anstrebt (Fig. $10.3 b$ ).

Da auch

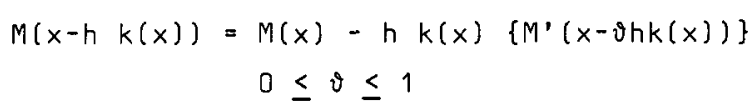

gilt und somit

$\frac{\cot \alpha(x)}{\cot \alpha \operatorname{dim}}=\frac{k(x)}{\cot \alpha_{\text {dim }}}=\frac{Q(x)}{Q(x-\vartheta h k(x))}$

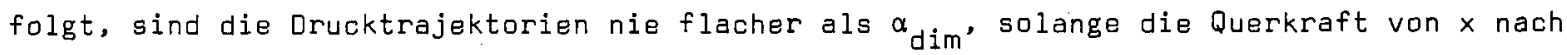
$x-h k(x)$ nicht abnimmt, d.h. solange wie in (10.20) vorausgesetzt $p \geq 0$ gilt. Eine genauere Untersuchung würde jedoch zeigen, dass das Ergebnis allgemein gilt, wenn in (10.20) bei negativem $p$ die zusätzliche Aufhängebewehrung berücksichtigt wird.

Sind aber die Drucktrajektorien nirgends flacher als bei der Dimensionierung angenommen, so können einerseits gemäss (10.17) die maximalen Betonspannungen höchstens im Verhältnis $\left(P_{y}+p\right) / P_{y}$ über den Werten liegen, die sich aufgrund eines kanstanten Betonspannungszustandes im Steg mit tana ${ }_{\text {dim }}$ aus der nominellen Schubspannung errechnen. Wird mit in Längsrichtung konstantem tan $\alpha_{\text {dim }}$ dimensioniert, so können also durch die nominelle Schubspannung nicht kontrollierte Spannungskonzentrationen im Stegbeton nur in den in Abschnitt 10.3 behandelten Bereichen bei Einzellasten und Auflagern auftreten. Andererseits kann aber auch die Flanschzugkraft nicht grösser sein als nach der Balkentheorie (Fachwerkmodell) berechnet. Dies ist in Fig. 10.3 c für den im Beispiel behandelten Fall $p \geq 0$ veranschaulicht.

Diese Ergebnisse fussen auf der Annahme, dass sich der Steg unter der Bemessungsbruchlast im Regime I befindet. Damit wird vorausgesetzt, dass die Druckresultierende $z_{u}$ oder $z_{0}$ vom Druckflansch allein aufgenommen werden kann, und die Neutralaxe im Bruchzustand im Druckflansch und nicht im Steg liegt (siehe auch Abschnitt 9.5). 
Es wird ein I-, T- oder symmetrischer Kastenträger mit gerader, einseitiger Voute untersucht, dessen Stegscheibe im Regime I plastifiziert ist (Fig. 10.5 a). Dabei wird wieder angenommen, dass die Bügelfliesskraft $P_{y}$ und die verteilte Belastung $p$ zumindest stückweise konstant sind. Die Längsarmierung sei in den Flanschen angeordnet.
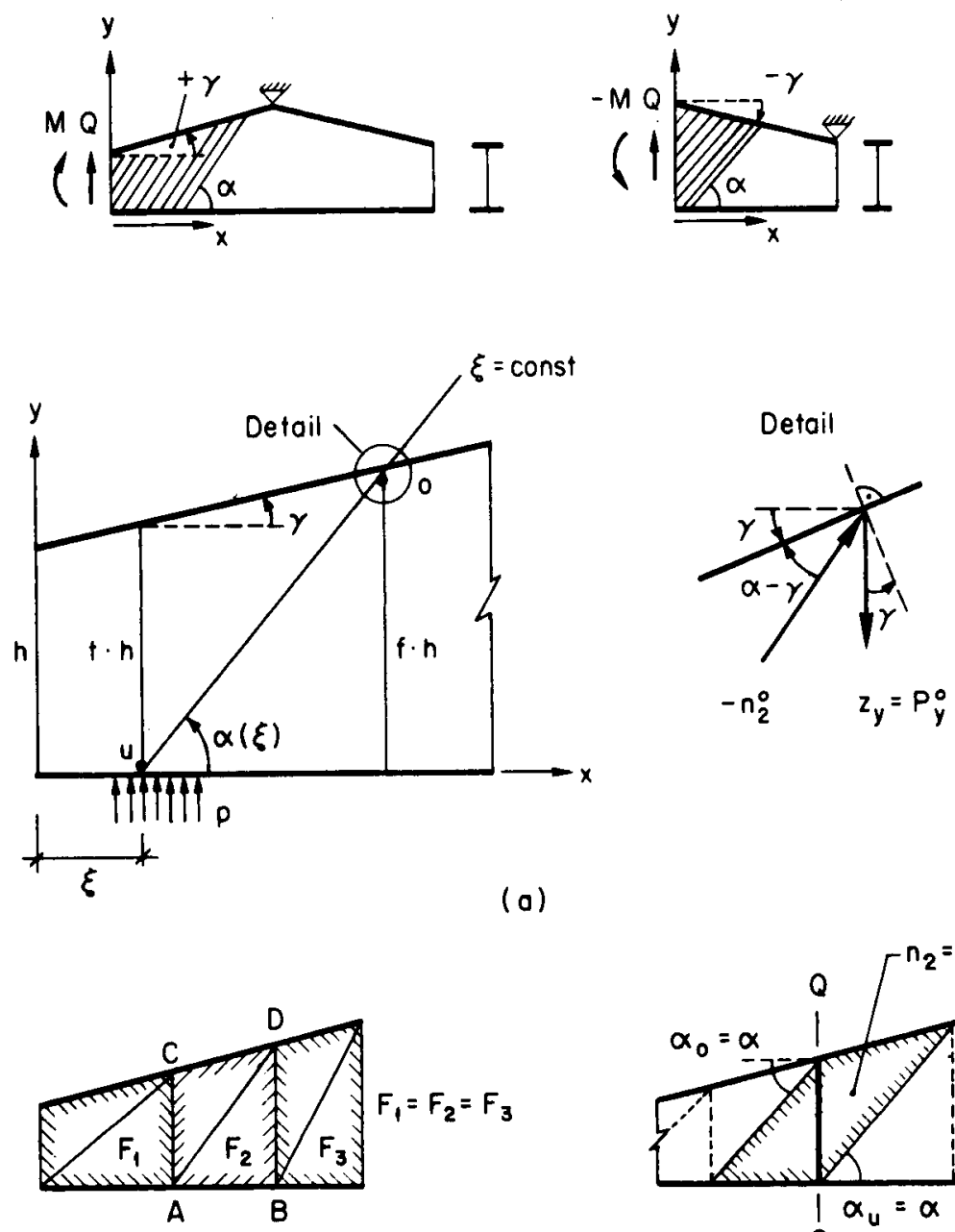

(b)
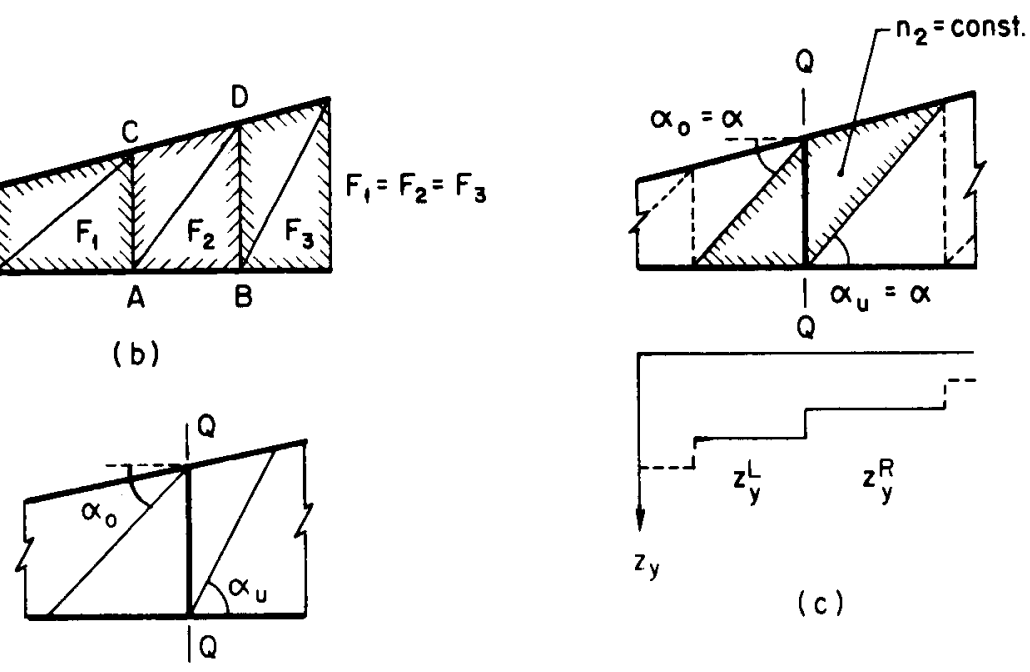

(c)

(d)

Fig. 10.5 Allgemeine Stegspannungszustände im Regime I für Vouten 
Bezeichnet $h$ die Höhe des Querschnittes $x=0$ und $t \cdot h$ bzw. f $h$ diejenige der Querschnitte, in welchen die betrachtete Drucktrajektorie $\xi$ = const. den unteren bzw. oberen Flansch schneidet (Fig. 10.5 a), so gilt

$t=1+\tan \gamma \xi / h$,

$f / t=1 /(1-\cot \alpha(\xi) \tan \gamma)$.

Die Beziehung zwischen den Betondruckspannungen der Trajektorie $\xi$ = const. am obern und untern Flansch lautet mit (9.22)

$n_{2}^{0}(\xi)=n_{2}^{u}(\xi) \frac{1}{1+n_{0} k^{\prime}(\xi)}$

Man erhält eine wesentlich einfachere Differentialgleichung, wenn statt der Funktion $\cot \alpha(\xi)=k(\xi)$ die Funktion $f(t)$, d.h. die Beziehung zwischen den Querschnittshöhen am untern und obern Trajektorienende gesucht wird. Dazu ist die Ableitung $\partial \cot \alpha(\xi) / \partial \xi=k^{\prime}(\xi)$ in $(10.25)$ durch $f, t$ und $f^{\prime}=\partial f / \partial t$ auszudrücken. Mit $(10.25)$ folgt

$$
\begin{aligned}
\cot \alpha & =\cot \gamma(1-t / f(t)) \\
\frac{\partial \cot \alpha}{\partial \xi} & =\frac{\partial \cot \alpha}{\partial t} \frac{\partial t}{\partial \xi}=\frac{1}{h}\left(-\frac{1}{f}+\frac{t}{f^{2}} f^{\prime}\right)
\end{aligned}
$$

Einsetzen von $(10.27)$ und $\eta_{0}=f \cdot h$ in $(10.26)$ ergibt

$\frac{n_{2}^{u}}{n_{2}^{0}}=1+\eta_{0} \frac{\partial \cot \alpha}{\partial \xi}=\frac{t}{f} f^{\prime}$.

Da am obern und untern Stegrand die Normalkomponente der totalen Scheibenrandspannung verschwinden bzw. gleich - $p$ sein muss, folgt für die Betondruckspannung auch (Fig. 10.5 a)

$$
\begin{aligned}
& n_{2}^{u}(\xi)=-\left(P_{y}+p\right)^{u} / \sin ^{2} \alpha(\xi), \\
& n_{2}^{0}(\xi)=-P_{y}^{0} \cos ^{2} \gamma / \sin ^{2}(\alpha(\xi)-\gamma), \\
& \text { oder mit } k=\left(P_{y}+p\right)^{u} / P_{y}^{0} \text { und }(10.25 b) \\
& \frac{n_{2}^{u}}{n_{2}^{0}}=k \frac{\sin ^{2}(\alpha-\gamma)}{\sin ^{2} \alpha \cos ^{2} \gamma}=k(1-\operatorname{cotatan} \gamma)^{2}=k \frac{t^{2}}{f^{2}} .
\end{aligned}
$$

Mit (10.28) und (10.30) lautet die gesuchte Differentialgleichung für $f(t)$

$f^{\prime} f=k t$,

und die Integration ergibt

$\| f^{2}-k t^{2}=$ const. $\|$.

Für stückweise konstante Bügelfliesskraft $P_{y}$ und Belastung $p$ ist mit (10.32) auch für Vouten eine sehr einfache Beziehung gefunden, mit der - ausgehend von einer bekannten Drucktrajektorie (z.B. ein als massgebend angenommener Kollapsriss) - das Trajektorienfeld und mit (10.29) die maximalen Betonspannungen bestimmt werden können. Eine besonders einfache Deutung von (10.32) erlaubt der Fall $k=1\left(p=0, P_{y}=\right.$ const. $)$. Wegen $(f-t)(f+t)=$ const. 
variiert die Neigung der Betondrucktrajektorien offensichtlich derart, dass die Stegfläche ABCD zwischen den Querschnitten durch das untere bzw. obere Trajektorienende konstant bleibt (Fig. 10.5 b).

Die Verallgemeinerung des Fachwerkmodelles auf Vouten findet sich z.B. in [22]. Annahmen und Vorgehen bei der Ableitung der Formeln für die Armierungsbeanspruchung und die M-Q-Interaktion eines Querschnittes entsprechen wieder der einfachen Balkentheorie (s. Abschn. 6; Fig. 6.3). Die Gleichgewichtsbedingungen zwischen den inneren und äusseren Kräften eines Querschnittes werden formuliert. Die Annahme eines über die Steghöhe des betrachteten Querschnittes kanstanten Betonspannungszustandes ist verträglich mit den verallgemeinerten Verformungen (6.10). Die Formulierung des Gleichgewichtes zwischen Bügel- und Druckdiagonalenkräften entlang eines Schnittes parallel zur Balkenaxe entspricht der Elimination der nichtrelevanten Normalspannung quer zur Balkenaxe: $N_{y}=P_{y}+n_{2} \sin ^{2} \alpha=0$.

Hier liegt der grundsätzliche Unterschied zwischen Scheiben- und einfacher Balkentheorie. Im Rahmen der letzteren wird die vom Steg übertragene Querkraft über den Ansatz $N_{y}=0$ und die Fliessbedingung (2.7 I) mit der Bügelfliesskraft verknüpft, die in den Gleichgewichtsbedingungen des Querschnittes nicht vorkommt. Dieser Zusammenhang ist somit in der einfachen Balkentheorie unabhängig vom Voutenanzug. Man beachte, dass mit $N_{y}=0$ die Randbedingungen am geneigten Stegrand nicht erfüllt werden. Nur die Schiefstellung der Flanschkräfte im betrachteten Querschnitt wird berücksichtigt nicht jedoch der Einfluss der Voute auf den Stegspannungszustand. Dieses Vorgehen wird im folgenden kurz als Fachwerkmodell bezeichnet.

Leitet man dagegen Interaktionsformeln mit Hilfe von Stegspannungsfeldern her, die in der Querschnittsumgebung statisch zulässig sind, so werden die Betonspannungen und mit ihnen die vom Steg übertragene Querkraft über die Randbedingungen (10.29) mit den Bügelkräften verknüpft. Da die physikalisch sehr anschaulichen Kollapsrissmechanismen als Scheibenmechanismen die Querschnittsumgebung ebenfalls einbeziehen, werden sie sich nur noch in Einzelfällen mit den verallgemeinerten Verformungen der einfachen Balkentheorie assaziieren lassen. Die Zusammenhänge werden durch die folgenden, später benötigten Beziehungen noch verdeutlicht.

Fragt man vorerst nach dem Querkraftverlauf, der für konstante Bügelfliesskraft $z_{y}=P_{y}=$ const. (in der Praxis ist.die Bügelfliesskraft immer stückweise konstant) zu einem konstanten Betonspannungszustand $\alpha=$ const. im Steg führt, so folgt aus (10.28) und (10.30) sofort

$1 / K=P_{y} /\left(P_{y}+P\right)=(1-\operatorname{cotatan} \gamma)^{2}$,

wobei die Vorzeichenkonventionen Fig. 10.5 a zu entnehmen sind. Für gegebene Werte $P_{y}, p, \alpha$ kann man (10.33) auch als Gleichung für den "idealen" Voutenanzug tany auffassen. Entspricht der Querkraftverlauf nicht (10.33), so liegen vollständig analoge Verhältnisse vor wie in einem Träger konstanter Höhe unter nicht konstanter Querkraft. Ein über die Steghöhe konstanter Betanspannungszustand ist wie beim prismatischen Träger (Fig. 10.3 a) höchstens noch in isolierten Querschnitten möglich im Zusammenhang mit Spannungszuständen, die als diskrete Fachwerke mit gleichmässig beanspruchten, bandartigen Bügeln und Druckdiagonalen aufgefasst werden können. Ist insbesondere die Querkraft konstant, so folgt aus (10.33) für das Verhältnis der Bügelzugkräfte $z_{y}^{L}, z_{y}^{R}$ links und rechts eines solchen Querschnittes (Fig. $10.5 \mathrm{c}$ ) $z_{y}^{R} / z_{y}^{L}=(1-\operatorname{cotatan} \gamma)^{2}$

Im allgemeinen ist jedoch von einem nicht konstanten Betonspannungszustand im Steg auszugehen und es interessiert dann die Beziehung zwischen den Neigungen $\alpha_{u}$, $\alpha_{0}$ der Betondruck- 
trajektorien am untern bzw. obern Rand des betrachteten Querschnittes (Fig. 10.5 d).

Aus (10.32) und (10.25b) folgt für zumindest je links und rechts des Querschnittes konstante Bügelzugkraft und verteilte Belastung:

$$
\begin{aligned}
f^{2}-k t^{2} & =1^{2} /\left(1-\cot \alpha_{u} \tan \gamma\right)^{2}-k \cdot 1^{2} \\
& =1^{2}-k\left(1-\cot \alpha_{0} \tan \gamma\right)^{2},
\end{aligned}
$$

$1 /\left(1-\cot \alpha_{U} \tan \gamma\right)^{2}+k\left(1-\cot \alpha_{0} \tan \gamma\right)^{2}=1+k$

Insbesondere folgt für konstante Querkraft und Bügelzugkraft $\left(p=0, z_{y}^{L}=z_{y}^{R}=P_{y}\right) \dot{m i t} k=1$

$1 /\left(1-\cot \alpha_{U} \tan \gamma\right)^{2}+\left(1-\cot \alpha_{0} \tan \gamma\right)^{2}=2$

Die Ableitung der M-Q-Interaktion im Regime I für einen Querschnitt im Bereich einer Voute ist in Fig. 10.6 zusammengestellt. Dabei wird vorerst vorausgesetzt, dass der Voutenanzug $r$ und die Trajektorienneigungen $\alpha_{0}, \alpha_{u}$ wie in Fig. 10.6 dargestellt positiv sind. Damit ist insbesondere auch die vom Steg übertragene Querkraft positiv. Die Beziehungen zwischen Flanschzugkräften $z_{u}, z_{0}$, Bügelbeanspruchung $z_{y}$ und Schnittkräften $M$, $Q$ eines Querschnittes aufgrund der in der Querschnittsumgebung durch (10.32) gegebenen Betonspannungszustände im Steg können natürlich wie in Abschnitt 10.1 (Gleichung (10.9)) direkt aus dem Querschnittsgleichgewicht ermittelt werden. Da die Randspannungen eines beliebigen, aus dem plastifi-

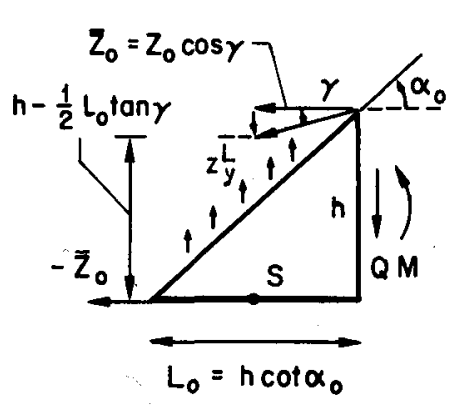

(a)

$$
\begin{aligned}
& \bar{z}_{0}=\frac{-M+\frac{1}{2} Q L_{0}}{h-\frac{1}{2} L_{0} \tan \gamma} \\
& z_{y}^{L}=\left(Q+\bar{Z}_{o} \tan \gamma\right) / L_{0} \\
& \bar{z}_{0}=\bar{Z}_{\text {of }}, z_{y}^{L}=P_{y}: \\
& \bar{Z}_{\text {of }}=-\frac{M}{h}+\frac{Q_{\text {eff }}^{2}}{2 P_{y} h} \\
& Q_{\text {off }}=Q+\bar{Z}_{\text {of }} \text { ton }, \quad \text { tan } \alpha_{0}=\frac{P_{y} h}{Q_{\text {eff }}}
\end{aligned}
$$

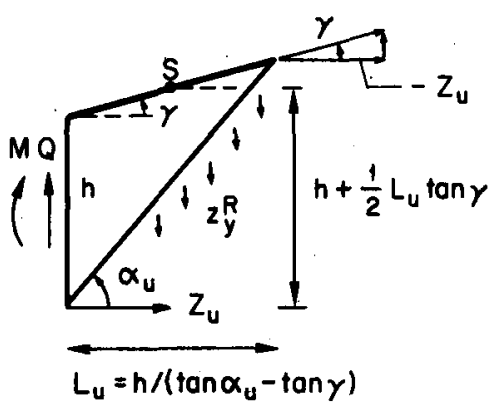

(b)

$$
\begin{array}{ll}
Z_{u}=\frac{M+\frac{1}{2} Q L_{u}}{h+\frac{1}{2} L_{u} \tan \gamma} \quad & L_{a}=\dot{\omega}\left(M+Q L_{u}\right) \\
L_{d}=\dot{\omega}\left\{Z_{u f}\left(h+L_{u} \tan \gamma\right)+\frac{1}{2} P_{y} L_{u}^{2}\right\} & M=Z_{u f}\left(h+L_{u} \tan \gamma\right)+\frac{1}{2} P_{y} L_{u}^{2}-Q L_{u} \\
Z_{y}^{R}=\left(Q-Z_{u} \tan \gamma\right) / L_{u} & \frac{\partial M}{\partial L_{u}}=0: \quad L_{u}=\left(Q-Z_{u f} \tan y\right) / P_{y} \\
Z_{u}=Z_{u f}, Z_{y}^{R}=P_{y}: & Z_{u f}=\frac{M}{h}+\frac{Q_{\text {eff }}^{2}}{2 P_{y} h} \\
Q_{e f f}=Q-Z_{u f} \text { tany, } \tan _{u}=\tan \gamma+\frac{P_{y} h}{Q_{e f f}}
\end{array}
$$

(c)

Fig. $10.6 \quad M-Q-$ Interoktion für die einseitige Voute 
zierten Bereich geschnittenen "free body" wegen (9.1) ein Gleichgewichtssystem bilden, leitet man sie einfacher und anschaulicher aus dem Gleichgewicht der Dreiecke ab, die durch den betrachteten Querschnitt und die vom unteren bzw. oberen Querschnittsrand ausgehenden Drucktrajektorien $\alpha_{u}, \alpha_{0}$ begrenzt werden (Fig. $10.5 \mathrm{~d}$ ). Die Herleitung erfolgt für eine Zone konstanter Bügelfliesskraft $P_{y}=$ const. und - um den Vergleich mit dem Fachwerkmodell zu ermöglichen - konstanter Querkraft $(P=0)$. Die Bügelzugkraft muss deshalb im Kollapszustand über den massgebenden Kollapsriss entlang der Trajektorie $\alpha_{u}$ oder $\alpha_{0}$ konstant sein. Wird ebenfalls auf der anderen Querschnittsseite eine konstante Bügelzugkraft angenommen, so ist'das Drucktrajektorienfeld zwischen den Trajektorien $\alpha_{u}$, $\alpha_{0}$ mit (10.35) und $k=z_{y}^{L} / z_{y}^{R}$ durch (10.32) gegeben. Die maximalen Betonspannungen treten am obern oder untern Querschnittsrand auf und sind deshalb über die Randbedingungen ebenfalls unmittelbar durch die Grössen $\alpha_{u}, \alpha_{0}$, $z_{y}^{L}$, $z_{y}^{R}$ von Fig. 10.6 bestimmt.

Die Gleichungen für die Flansch- und Bügelzugkräfte in Fig. 10.6 a,b sind leicht verständlich. Während das Horizontalgleichgewicht schon in der Zeichnung berücksichtigt ist und $z_{y}^{L}, z_{y}^{R}$ aus dem Vertikalgleichgewicht folgen, ergeben sich $Z_{u}$, $Z_{0}$ aus der Momentenbedingung um den Schwerpunkt $S$ der Bügelzugkraft auf der Höhe des jeweils gegenüberliegenden Flansches. Die Formeln von Fig. 10.6 a und b lassen sich vollkommen analog schreiben, wenn statt der Neigungen die horizontalen Längen $L_{u}$, $L_{0}$ der Drucktrajektorien $\alpha_{u}$, $\alpha_{0}$ als Variable eingeführt werden. Diese Wahl vereinfacht zudem im Falle, massgebender unterer Längsarmierung (Fig. 10.6 b,c) den Uebergang zur Interaktionsbeziehung wesentlich. Setzt man zur Kontrolle $\alpha_{U}=\alpha_{0}=\alpha$ bzw. $z_{y}^{L}=z_{y}^{R}=P_{y}$, so verifiziert man nach einiger Rechnung wieder (10.34) bzw. (10.36).

Drückt man in den Gleichungen für $Z_{u}$, $Z_{0}$ die Variablen $L_{u}$, $L_{0}$ durch $\alpha_{u}$, $\alpha_{0}$ aus, so stellt man fest, dass sich nicht nur für $z_{0}$, sondern auch für $z_{u}$ genau gleiche Ausdrücke ergeben wie beim Fachwerkmodell [22], wenn dort $\alpha=\alpha_{0}$ bzw. $\alpha=\alpha_{u}$ gesetzt wird. Andererseits ist die im Fachwerkmodell [22] verwendete Beziehung für die Bügelzugkraft für $\alpha=\alpha_{0}$ mit dem Ausdruck für die Bügelzugkraft links $z_{y}^{L}$ identisch. Die für massgebende obere Flanscharmierung resultierende M-Q-Interaktion (Fig. 10.6 a) unterscheidet sich daher auch nicht von derjenigen des Fachwerkmodelles. Der Berechnung der Betonspannungen darf in diesem Fall ein über den Querschnitt konstanter Spannungszustand $\alpha_{u}=\alpha_{0}=\alpha$ zugrunde gelegt werden; die Bügelspannungen rechts liegen dann im Verhältnis (10.34) unter der Fliessgrenze. Da die Interaktionsbeziehungen Fig. 10.6 a natürlich auch über einen Kollapsrissmechanismus entlang der Trajektorie $\alpha_{0}$ folgt, darf dieser mit den verallgemeinerten Verformungen (6.10) der Balkentheorie assoziiert werden.

Wird jedoch im betrachteten Querschnitt die Längsarmierung im untern Flansch massgebend, so kann der Kollapszustand bei nicht massgebender Betonfestigkeit erst dann erreicht sein, wenn die Bügelarmierung rechts des Querschnittes fliesst und sich ein Kollapsiss entlang der Trajektorie $\alpha_{u}$ öffnen kann. Geht man wie in Fig. 10.6 b tatsächlich vom Fliessen der Bügel rechts des Querschnittes aus, so ergibt sich eine Interaktionsbeziehung, die natürlich auch über die Mechanismusmethode mit einem sich entlang der Trajektorie $\alpha_{u}$ öffnenden Kollapsriss folgt (Fig. $10.6 \mathrm{C}$ ). Im Fachwerkmodell dagegen wird die Drucktrajektorienneigung in der Gleichung $z_{u}=z_{u f}$ offensichtlich mit der Bedingung eliminiert, dass die Bügelarmierung links des Querschnittes fliesst, $z=P_{y}$. Unter der Annahme eines über den Querschnitt konstanten Betonspannungszustandes kann natürlich die Bügelarmierung rechts wegen (10.34) auch nie die Fliessgrenze erreichen. Die Interaktionsbeziehung des Fachwerkmodelles unterscheidet sich von derjenigen gemäss Fig. 10.6 b,c im Ausdruck für die effektive Querkraft, der durch

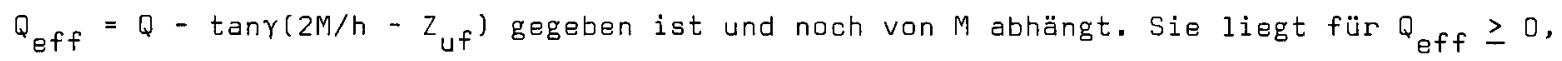
$\gamma \geq 0$ auf der sicheren Seite, die zugehörigen verallgemeinerten Verformungen haben aber keine physikalische Bedeutung mehr, weil ihr noch kein Scheibenmechanismus entspricht. 
Die Interaktionsformeln von Fig. 10.6 gelten natürlich nur, solange die Betondruckfestigkeit nirgends überschritten wird. Kritisch ist vor allem der Fall massgebender Bügelarmierung rechts (Fig. $10.6 \mathrm{~b}, \mathrm{c}$ ), weil der Betonspannungszustand im Steg wegen (10.34) nicht mehr kanstant sein kann. Fliesst die Bügelarmierung rechts des Querschnittes, so liegt auch diejenige links an der Fliessgrenze, $z_{y}^{L}=z_{y}^{R}=P_{y}$. Mit (10.36) wird dann die Drucktrajektorienneigung $\alpha_{0}$ am oberen Querschnittsrand wesentlich flacher als die Neigung $\alpha_{u}$ des massgebenden Kollapsrisses. Für cot $\alpha_{u}=5 / 3, \tan \gamma=0.1$ ergibt sich zum Beispiel cot $\alpha_{0}=2.5$, und die maximale Betonspannung im Steg am obern Querschnittsrand wird rund $3.5 \mathrm{mal}$ grösser als der Wert, der sich aus $N_{y}=P_{y}+n_{2} \sin ^{2} \alpha=0$ mit $\alpha=\alpha_{u}$ errechnet.

Man überprüft leicht, dass der Fall $Q_{\text {eff }} \leq 0, \gamma \geq 0$ dem Fall $Q_{\text {eff }} \geq 0, \gamma \leq 0$ entspricht, der in Fig. 10.5 a oben rechts dargestellt ist, und für den die Formeln in Fig. 10.6 offensichtlich weiterhin gelten. In diesem Fall liegt das Fachwerkmodell nun auf der unsicheren Seite. Bei massgebender unterer Längsarmierung wird die Beanspruchung der Bügel rechts unterschätzt. Bei massgebender oberer Längsarmierung dagegen wird die maximale Betonspannung unterschätzt, da der Betonspannungszustand im Steg nicht mehr konstant sein kann.

Als Schlussergebnis, das auch für zweiseitige Vouten gilt, kann falgendes festgehalten werden. Solange bei der querschnittsweisen Bemessung die Betonspannungen im Steg nur über die nominelle Schubspannung kontrolliert werden, sollte wie beim Fachwerkmodell von konstanten Betonspannungszuständen im Steg ausgegangen werden. Dagegen ist die Bügelzugkraft aus der kritischeren Randbedingung am oberen oder unteren Stegrand zu gewinnen. Die resultierenden Interaktionsbeziehungen sind dann sowohl bezüglich der Armierung als auch des Betonspannungszustandes auf der sicheren Seite, im allgemeinen entspricht ihnen aber noch kein Scheibenmechanismus.

Zuro Schluss sei noch auf ein mit dem Thema dieses Abschnittes verwandtes Prablem hingewiesen. Aus der Sicht statisch zulässiger Spannungszustände muss man sich fragen, ob ein Träger konstanter Höhe, der als Hauptlängsarmierung ein schräges Spannkabel und auf der Balkenunterseite nur eine sehr schwache, konstruktive Längsarmierung aufweist, nicht auch dann als Voute im Sinne von Fig. 10.5 a oben rechts zu betrachten sei, wenn die Bügelarmierung bis zur Balkenunterseite geführt ist. Es stellt sich somit die für die Praxis relevante Frage, welche statische Höhe einzusetzen sei zur Berechnung der Bügelarmierung und der nominellen Schubspannung. Sie ist bisher noch nicht befriedigend beantwortet worden.

\subsection{Krafteinleitungen}

Im Bereich von Lasteinleitungsstellen wird die Beanspruchung der Armierung unter rechnerischer Bruchlast vielfach aufgrund von Ueberlegungen an fächerartigen Fachwerken mit diskreten Druckdiagonalen abgeschätzt, die unter der Einzellast bzw. am Einspannquerschnitt in einem Punkt zusammenlaufen (Fig. 10.7 a), [z.B. 22]. Aus dieser Sicht ist z.B. im Einspannquerschnitt C-C Fig. 10.7 a links keine zusätzliche Längsarmierung infolge Querkraft notwendig. Die Annahme, dass der Beton die entsprechenden Beanspruchungen aufnehmen kann, soli im folgenden theoretisch etwas genauer untersucht werden.

Eetrachtet man die Fächer Fig. 10.7 a als Trajektorienfeld eines Scheibenspannungszustandes, so ist dieser natürlich der unendlich grossen Betonspannungen wegen nicht stabil. Andererseits stellen die von den Querschnitten A-A, B-B, C-C (Fig. 10.7 a) ausgehenden Geraden die ungünstigsten "Kollapsrisse" im Sinne der auf diese Querschnitte angewandten "Querschnitts"- 

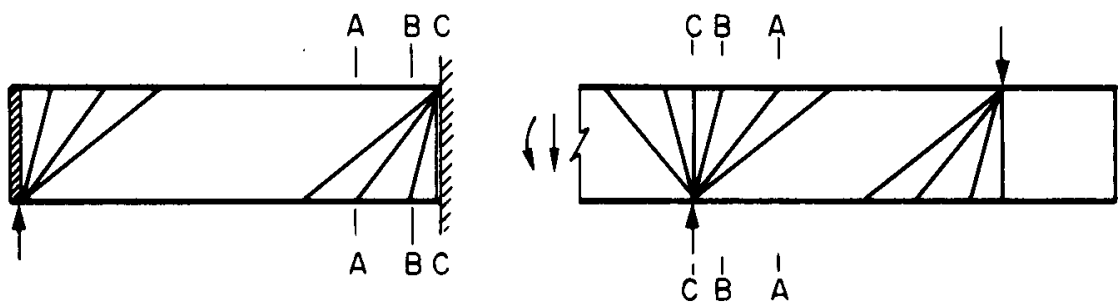

(a) Diskrete Druckdiagonalen z.8. nach $[22]$
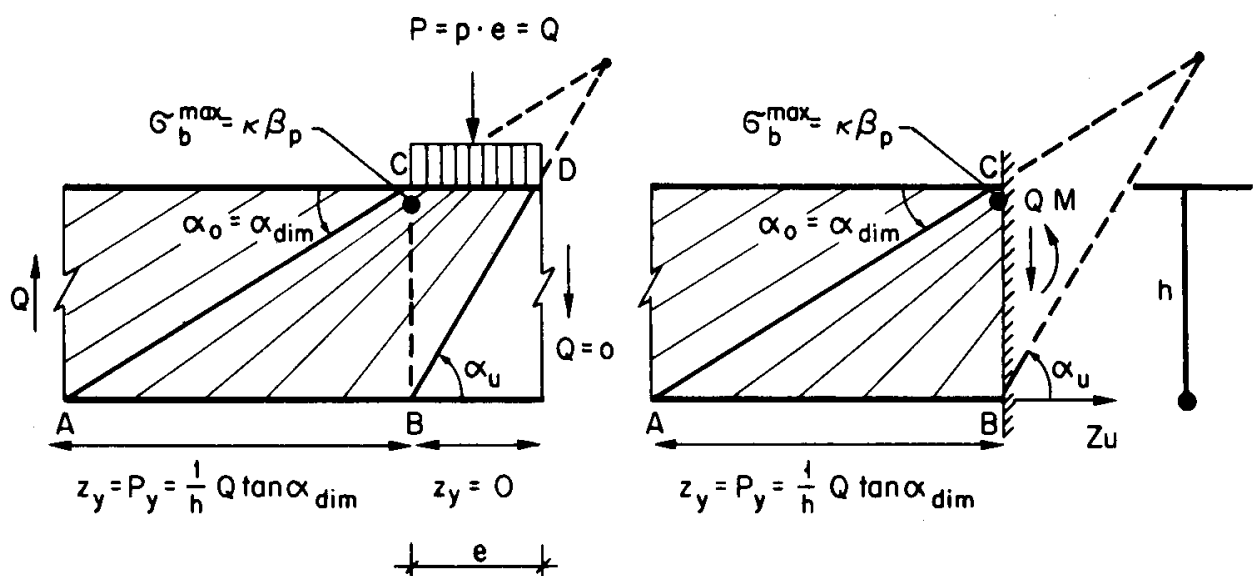

$e=h \cot \alpha_{u}$

$z_{u}=\frac{M}{h}+\frac{1}{2} Q \cot \alpha_{u}$

\begin{tabular}{|c||c|c|c|}
\hline \multicolumn{1}{c}{$\cot \alpha_{u}$ für $\sigma_{b}^{\max }=\kappa \beta_{p}$} \\
\hline $\tan _{\text {dim }} / \kappa \beta_{p}$ & $\frac{60}{225}$ & $\frac{60}{0.7 \cdot 225}$ & $\frac{50}{0.7 \cdot 225}$ \\
\hline $3 / 5$ & 1.01 & 1.44 & 1.20 \\
1 & .53 & .76 & .64 \\
\hline
\end{tabular}

(b) Krofteinleitung über zentrierte Fächer

Fig. 10.7 Krafteinleitungen

Mechanismen gemäss Fig. 6.8 dar, wenn die kinematischen Randbedingungen an der Einspannstelle bzw. die äussere Arbeit der Einzellast berücksichtigt wird. Aus der Sicht der Scheibentheorie entspricht obiges Vorgehen deshalb der Mechanismusmethode mit der Klasse der mit dem Regime I verträglichen Mechanismen.

Im Falle der direkten Lasteinleitung interessiert, wie gross die minimale Lasteinleitungslänge e sein muss, im Falle der indirekten Krafteinleitung (Einspannstelle, Lasteinleitung über Querträger oder Lagerendblöcke, die in der Stegscheibenebene als starr angesehen werden dürfen) wie gross die Fliesskraft der Hauptlängsarmierung im "Einspann"-Querschnitt sein muss, damit unter der Bemessungsbruchlast stabile, statisch zulässige Stegspannungszustände im Regime I existieren. 
Eine vernünftige Abschätzung dieser Werte erhält man schon mit einem zentrierten Fächer, dessen Zentrum aus der Bedingung bestimmt wird, dass die maximale Betonspannung im Steg die massgebende Betondruckfestigkeit $K \beta_{p}$ gerade erreicht (Fig. 10.7 b). Die direkte Krafteinleitung entspricht dann für konstante Bügelfliesskraft einer konstanten verteilten Belastung p. Die maximale Betonspannung im Punkt $C$ rechts beträgt gemäss (10.16a)

$\sigma_{b}=\frac{Q}{b_{0} h} \tan \alpha_{u}\left(1+\cot ^{2} \alpha_{0}\right)$

$\alpha_{0}$ ist unter der Bemessungsbruchlast durch die zur Dimensionierung der Schubarmierung verwendete "Diagonalenneigung" $\alpha_{\text {dim }}$ gegeben, während $\alpha_{u}$ die gesuchte Grösse ist, aus der sich die Lasteinleitungslänge e (Fig. 10.7 b, links)

$e=h \cot \alpha_{U}$,

oder mit (10.12) die Flanschzugkraft $Z_{U}$ im Einspannquerschnitt (Fig. 10.7 b, rechts) errechnen,

$z_{u}=\frac{M}{h}+\frac{1}{2} Q \cot \alpha_{u}$

Mit $\sigma_{b}=k \beta_{p}, \alpha_{0}=\alpha_{d i m}$ folgt aus $(10.37)$

$\left.\begin{array}{l}\cot \alpha_{u}=\frac{\tau}{k \beta_{p}}\left(1+\cot ^{2} \alpha_{d i m}\right), \\ \text { mit } \tau=Q / b_{0} h .\end{array}\right\}$

(10.40) drückt cota durch die in Normen vorgeschriebenen oder begrenzten Grössen nominelle Schubspannung $\tau$, "Diagonalenneigung" $\alpha_{\text {dim }}$ aus und ist in Fig. 10.7 b an den oberen Schubspannungsgrenzen gemäss Richtlinie $34[29]$ für einen $B H 300\left(\beta_{p}=225 \mathrm{~kg} / \mathrm{cm}^{2}\right)$ und für $\tan _{\mathrm{dim}}=3 / 5 \mathrm{bzw} .1$ ausgewertet. $k=0.7$ ist wieder eine konservative Schätzung für das Verhältnis von statischer zu nomineller Prismenfestigkeit (siehe Abschnitt 7.3).

Kleinere Werte für e und $Z_{u}$ erhält man mit einer Folge von $n$ zentrierten Fächern (Fig. 10.8 links), welche für $n \rightarrow \infty$ in einen nicht zentrierten Fächer übergeht. Die Stegbetonspannung liegt dann über den ganzen Einspannquerschnitt (Fig. 10.8 a) bzw. über die gesamte Lasteinleitungslänge CD (Fig. 10.8 b) an der Druckfestigkeitsgrenze.

Die Beziehung (10.12) für $Z_{u}$ im Einspannquerschnitt (Fig. 10.8 a) gilt für nicht zentrierte Fächer nicht mehs und $Z_{u}$ wird am einfachsten von $A$ aus aufintegriert $\left(Q=\right.$ const., $P_{y}=$ const.) $Z_{u}=\frac{1}{h}\left(M-Q h \cot \alpha_{0}\right)+\frac{1}{2} Q \cot \alpha_{0}+I$,

$I=P_{y} \int_{-h \cot \alpha_{0}}^{0} \cot \alpha(\xi) d \xi=P_{y} h \cot \alpha_{0} \cot \alpha_{m}=Q \cot \alpha_{m}$,

$z_{u}=\frac{M}{h}+\frac{1}{2} Q \cot \alpha_{u}^{*}$

$\cot \alpha_{u}^{*}=2 \cot \alpha_{m}-\cot \alpha_{0}$

$\cot \alpha_{u}^{*}$ ist lediglich eine fiktive Grösse zur Berechnung von $Z_{u}$. Das Trajektorienfeld $\operatorname{cota}(\xi)=$ $k(\xi)$ im Bereich $A B C$ von Fig. 10.8 a ist gemäss Abschnitt 11 (Gleichungen (11.35), (11.36)) 


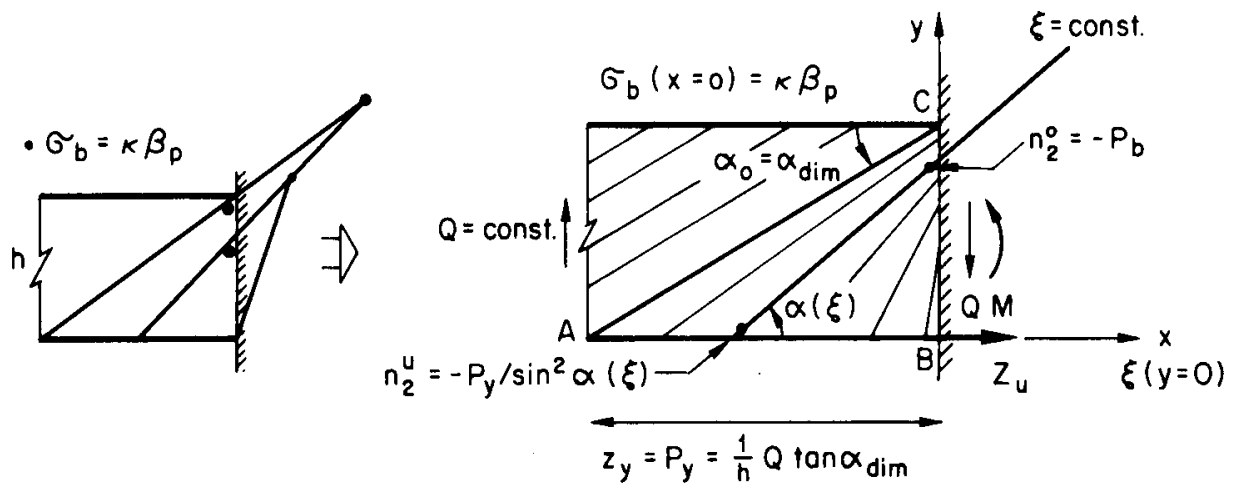

(a) $\cot \alpha_{u}^{*} ; \quad\left(Z_{u}=\frac{M}{h}+\frac{1}{2} Q \cot \alpha_{u}^{*}\right)$

\begin{tabular}{|c|c|c|c|}
\hline $\tan \alpha_{\mathrm{dim}}$ & $\frac{60}{225}$ & $\frac{60}{0.7 \cdot 225}$ & $\frac{50}{0.7 \cdot 225}$ \\
\hline $3 / 5^{\circ}$ & 47 & .94 & .63 \\
\hline 1 & 28 & 50 & 36 \\
\hline
\end{tabular}

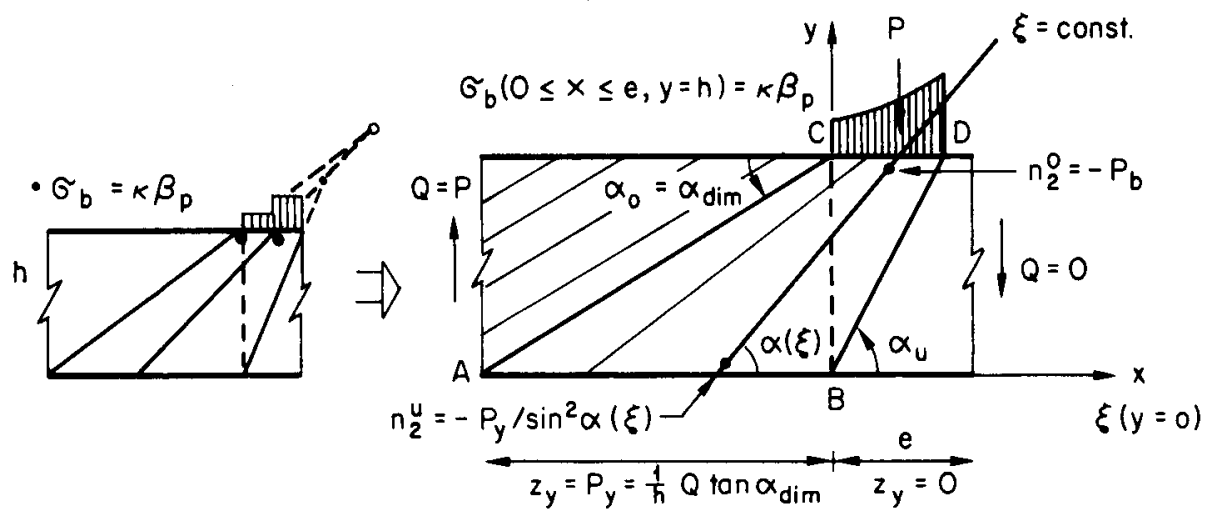

(b)

\begin{tabular}{|c|c|c|c|}
\hline $\begin{array}{l}\tau / \alpha \beta_{p} \\
\tan \alpha_{d i m}\end{array}$ & $\frac{60}{225}$ & $\frac{60}{0.7 \cdot 225}$ & $\frac{50}{0.7 \cdot 225}$ \\
\hline $3 / 5$ & 69 & 1.23 & .90 \\
\hline 1 & 42 & 66 & 52 \\
\hline
\end{tabular}

Fig. 10.8 Krafteinleitungen (über nicht zentrierte Fächer) 
gegeben durch

$$
\left.\begin{array}{rl}
\frac{\cot \alpha(\bar{\xi})}{\cot \alpha_{K}} & =\left\{1+\frac{c}{\bar{\xi}^{P}}\right\}^{-\sqrt{2} ; \text { mit }} \\
\bar{\xi} & =-\xi /\left(h \cot \alpha_{0}\right), p=2(1-\bar{\mu}), \\
c & =\left(\cot \alpha_{K} / \cot \alpha_{0}\right)^{2}-1, \\
\bar{\mu} & =\frac{P_{y}}{P_{b}}=\frac{\mu \sigma_{f}}{\kappa \beta_{p}}, \\
\sin ^{2} \alpha_{K} & =\bar{\mu}, \quad \cot ^{2} \alpha_{K}=(1-\bar{\mu}) / \bar{\mu} .
\end{array}\right\}
$$

$\alpha_{K}$ ist die der theoretischen oberen Schubspannungsgrenze gemäss Abschnitt 7 entsprechende kritische Drucktrajektorienneigung. Für $\alpha_{\text {dim }}=\alpha_{0}>\alpha_{K}$ strebt $\alpha$ von A nach B gegen $90^{\circ}$. Die Grössen $\cot \alpha_{u}^{*}$ und $\cot \alpha_{m}$

$\cot \alpha_{m}=\cot \alpha_{K} \int_{0}^{1}\left\{1+c / \bar{\xi}^{p}\right\}^{-V / 2} d \bar{\xi}$

können mit

$\alpha_{0}=\alpha_{\text {dim }}, \quad \bar{\mu}=\bar{\tau} \tan \alpha_{d i m}, \quad \bar{\tau}=\frac{\tau}{k \beta_{p}}$

wieder als Funktion der nominellen Schubspannung und der zur Dimensionierung der Schubarmierung gewählten Diagonalenneigung berechnet werden. Die Resultate der Auswertung von (10.43), (10.41b) mittels Gauss'scher Quadratur sind aus der Tabelle in Fig. 10.8 a ersichtlich.

Für den Fall der direkten Krafteinleitung (Fig. 10.8 b) folgt aus (9.22) und den Randbedingungen für eine Trajektorie $\xi=$ const.

$\frac{n_{2}^{u}(\xi, n=0)}{n_{2}^{0}(\xi, n=h)}=\frac{P_{y}\left(1+k^{2}(\xi)\right)}{P_{b}}=1+h k^{\prime}(\xi)$.

Mit $\left(10.42\right.$ b) und den Abkürzungen $k_{K}=\cot \alpha_{K}, k_{0}=\cot \alpha_{0}, k_{u}=\cot \alpha_{u}$ folgt

$\frac{d k}{\left(k^{2}-k_{K}^{2}\right)}=\frac{\bar{\mu}}{h} d \xi$,
$\frac{k}{k_{K}}=\operatorname{Tanh}\left(\right.$ Const. $\left.-\bar{\mu} k_{K} \frac{\xi}{h}\right)$.

Die Konstante bestimmt sich aus der Randbedingung

$k=k_{0}$ für $\xi=0-h k_{0}$,

und die gesuchte Trajektorienneigung $\cot \alpha_{u}=k_{u}=k(\xi=0)$

ergibt sich zu

$k_{u}=k_{k} \operatorname{Tanh}\left\{A \Gamma \operatorname{Tanh}\left(k_{0} / k_{k}\right)-k_{0} k_{k} \bar{\mu}\right\}$, 
oder mit (10.44)

$k_{u}=k_{K} \operatorname{Tanh}\left\{\operatorname{ArTanh}\left(k_{d i m} / k_{K}\right)-\bar{\tau} k_{k}\right\}$.

Das Verhältnis $\cot \alpha_{u} / \cot \alpha_{0}$ wird gemäss (10.47) umso grösser, je näher die nominelle Schubspannung an der theoretischen oberen Schubspannungsgrenze gemäss Abschnitt 7 liegt $\left(\bar{\tau}^{2}=\right.$ $\bar{\mu}(1-\bar{\mu}))$ und erreicht an dieser den wert $1\left(\cot \alpha_{U}=\cot \alpha_{0}=\cot \alpha_{K} \geq 1\right)$. Die theoretische obere Schubspannungsgrenze gemäss Abschnitt 7 muss deshalb im Rahmen der Scheibentheorie bei direkter Krafteinleitung als oberer Grenzwert betrachtet werden, solange nicht nachgewiesen ist, dass auch in diesem Fall stabile, statisch zulässige spannungszustände im Steg existieren, die eine Lasteinleitung über vernünftige Längen möglich erscheinen lassen.

Mit (10.48), (10.42b) und (10.44) kann cota wieder als Funktion der nominellen Schubspannung und der zur Dimensionierung der Schubarmierung gewählten "Diagonalenneigung" ausgewertet werden. Die Ergebnisse sind in der Tabelle von Fig. 10.8 b zusammengestellt.

Die Resultate von Fig. 10.8 a zeigen, dass die Schubübertragung durch den Steg im Einspannquerschnitt wohl eine wesentlich kleinere zusätzliche Längsarmierung infolge Querkraft erfordert als im Feld. Bei voller Ausnützung der oberen Schubspannungsgrenze und Dimensionierung der Bügel mit tan ${ }_{d i m}=3 / 5$ kann letztere aber immer noch werte erreichen, wie sie im Feld bei der Dimensionierung mit tana ${ }_{d i m}=1$ nicht vernachlässigt werden. Wird diese zusätzliche Längsarmierung nicht eingelegt, so ergibt sich im ungünstigsten Fall (kleines Momenten-Schubverhältnis $M / Q h=3$; Dimensionierung mit tana $\left.{ }_{\text {dim }}=3 / 5, \tau=60 \mathrm{~kg} / \mathrm{cm}^{2} ; k=0.7\right)$ eine theoretische Reduktion des Traglastfaktors um 10\% auf der Basis der Beziehungen (10.41) bis (10.43). Entsprechendes gilt ebenfalls für indirekte Krafteinleitungen über Lagerendblöcke oder Querträger, sofern diese wegen ihrer Dimensionen oder wegen Symmetriebedingungen als in Stegscheibenebene starr angesehen werden dürfen. Der mit a ebenfal is bekannte Schubspannungsverlauf entlang des Querträgers gibt dann zusätzlich Anhaltspunkte über die Verteilung der Querträgerarmierung.

Im Falle der direkten Krafteinleitung (Fig. 10.8 b) kann die volle Biegetragfähigkeit des Querschnittes rechts der Last nur dann erreicht werden, wenn der Druckflansch die auf ihm aufgebrachte Einzellast bis zum Uebergang Flansch - Steg auf eine Länge verteilen kann, die im Steg noch stabile, statisch zulässige Spannungszustände zulässt. Die Tabellenwerte in Fig. 10.8 b für eindimensionale Druckspannungszustünde zeigen jedoch, dass diese minimal erforderliche Länge e für die Lasteintragung in den Steg bei voller Ausnützung der oberen Schubspannungsgrenze und für mit tan $\alpha_{\text {dim }}=3 / 5$ dimensionierte Bügel Grössenordnungen erreichen kann, die die "Lastverteilungskapazität" schlanker Druckflanschen überschreiten dürften. Gerade in diesem Fall spielt es auch keine grosse Rolle, dass die obere Randbedingung auf der Hähe der Druckflanschresultierenden statt im Uebergang Steg - Flansch formuliert wurde. Wesentlich kleinere Werte für e sind nur denkbar, wenn im Steg unter der Last ein zweidimensionaler Druckspannungszustend mit entsprechend erhöhter Betoridruckfestigkeit vorhanden ist. Die volle Biegetragfähigkeit kann dann aber theoretisch nicht mehr erreicht werden, weil die Zugkraft in der Längsarmierung unter der Last grässer sein muss als für reine Biegung, wo die Neutralachse im Bereich von Momentenspitzen normalerweise im Druckflansch liegt. Das meist nahe an der theoretischen Biegebruchlast liegende Versagen des Druckflansches auf Schub in der Nähe der Einzellast wird in Versuchsberichten ebenfalls als Biegeschubbruch bezeichnet.

Aus der Sicht dieser Arbeit sind also zwei Biegeschubbrucharten zu unterscheiden: Einerseits der Biegeschubbruch infolge zu schnell abgestufter Armierung, der auch unter der Annahme 
nicht massgebender Betondruckfestigkeit erfasst wird (Abschnitt 6.4), und andererseits der Biegeschubbruch im Krafteinleitungsbereich, wo das Problem der Schubtragfähigkeit des Steges nicht vom Problem der Einleitung der Last in den Druckflansch und van diesem in den Steg zu trennen ist. Während ausserhalb von Krafteinleitungsbereichen die Schubtragfähigkeit des Druckflansches gegenüber jener des Steges vernachlässigbar sein dürfte und deshalb in Richtlinie 34 auch nicht mehr berücksichtigt wird, gewinnt in Krafteinleitungszonen die Schubtragfähigkeit oder Lastverteilungskapazität des Druckflansches umso grössere Bedeutung, je kleiner die durch die Betondruckfestigkeit beschränkten Spannungskonzentrationsmöglichkeiten des Steges sind.

Biegeschubbrüche, die nicht auf zu schnell abgestufte Armierung zurückgeführt werden können, scheinen primär ein lokales Krafteinleitungsphänomen zu sein und sind auch durch lokale Massnahmen zu verhindern. Die Tabellenwerte zeigen, dass das Krafteinleitungsproblem erheblich entschärft wird, wenn in Bereichen unmittelbar neben grossen Einzellasten, wo zugleich die obere Schubspannungsgrenze voll ausgenützt ist, die Bügeil mit tana ${ }_{d i m}=1$ dimensioniert werden. 
11.1 Problemstellung

Als Beispiel für die Anwendung der Scheibentheorie auf Stahlbetonscheibenprobleme wird der "Shear Wall Coupling Beam 391" behandelt, der von Paulay im Rahmen eines umfangreichen Forschungsprojektes "The Coupling of Shear Walls" [44] getestet wurde. Als "Coupling Beams" werden die Verbindungselemente bezeichnet, die kompakte Hochhausscheiben zwischen Fenster- und anderen Deffnungen verbinden. Abmessungen, Beanspruchung. Armierung, Materialkennwerte und Rissebild im Kollapszustand sind für den "Coupling Beam 391" in Fig. 11.1 a zusammengestellt.
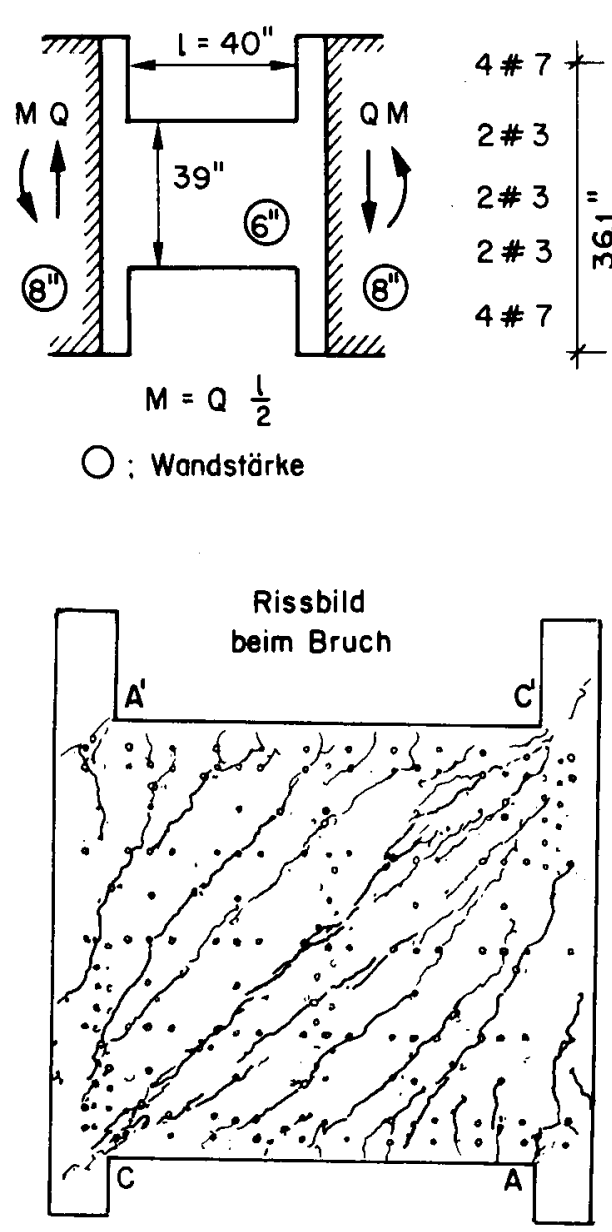

(b) Idealisierung

$$
\begin{aligned}
& Z_{f}=105 \text { Kips } \\
& P_{x} h=37.4 \text { Kips } \\
& P_{y}=3.11 \text { Kips } / \text { inch } \\
& P_{b}=\kappa \cdot 27.4 \text { Kips } / \text { inch } \\
& \left.\begin{array}{rl}
\kappa & =1: \text { mit } \\
\kappa & =2 / 3: \text { ohne }
\end{array}\right\} \text { Betonüberdeckung }
\end{aligned}
$$

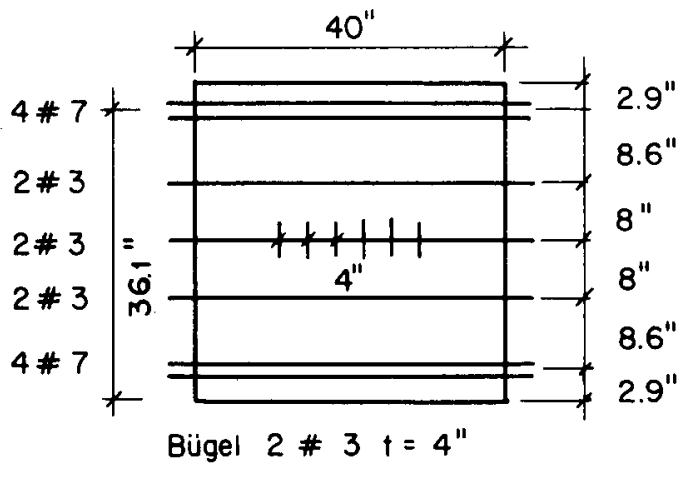

Materialkeninwerte

$$
\begin{aligned}
& \text { Stahl : \# } 7: \sigma_{f}=45.8 \mathrm{Ksi} \\
& \text { \# } 3: \sigma_{f}=59.0 \mathrm{Ksi}
\end{aligned}
$$

Beton: $\beta_{p}=4570$ psi

\section{Bruchlasten}

$$
\text { Theor. Biegebruchlast }
$$$$
Q_{u}=205 \text { Kips }
$$

Experimentelle Bruchlast

$$
Q_{u}=174.5 \text { Kips }
$$

\section{(a) Versuchsauslegung}

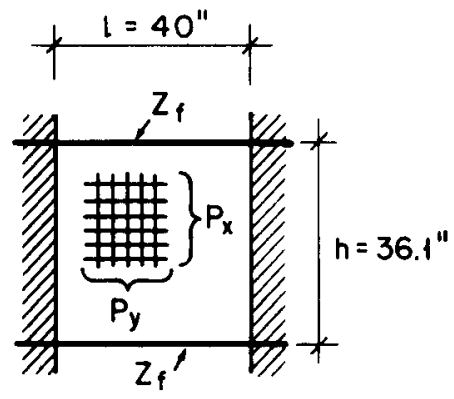

Fig. 11.1 Sheor Wall Coupling Beam 391 [44] 
Aus den Abmessungen ist sofort ersichtlich, dass der "Coupling Beam 391" selbst ebenfalls als Schubwand bzw. Scheibe betrachtet werden muss.

Dieser Versuch eignet sich deshalb besonders gut für den Vergleich von Theorie und Experiment, weil

- die Lasten über starre Endblöcke eingeleitet werden und somit keine Probleme mit konzentrierten Einzellasten auftreten;

- die Scheibe überall die gleiche Wandstärke aufweist;

- die vertikale Armierung so stark ist, dass die Betonzugspannungen beim Bruch kaum einen grossen Einfluss haben dürften;

- Die Scheibe überall gut durchgerissen ist, so dass sie beim Kollaps als vollplastifiziert betrachtet werden kann;

- die Symmetriebedingungen und die starren Endblöcke die möglichen Spannungs- und Geschwindigkeitsfelder beim Kollaps stark einschränken.

- Die Scheibe ebenfalls von Cervenka [46] mit einem nichtlinear-elastisch-plastischen "Finite Element"-Programm berechnet wurde.

Die theoretische reine Biegetraglast der scheibe beträgt $Q_{u}^{T h}=2 M_{u} / 1=205$ Kips, während beim rein statischen Versuch die Tragfähigkeit bei $Q_{U}^{\exp }=174.5 \mathrm{Kips}$, d.h. bei $85 \%$ der reinen Biegetragfähigkeit, erschöpft war. Beim Kollaps floss die Hauptlängsarmierung in den Endpunkten $A, A^{\prime}$ und die verteilte Armierung in der Umgebung der grossen Risse im Bereich der Scheibendiagonalen $C-C^{\prime}$.

Das idealisierte Tragsystem ist in Fig. 11.1 b dargestellt. Die theoretische Behandlung folgt dabei der in Schubliteratur üblichen Unterscheidung zwischen direkter Schubübertragung von Endquerschnitt zu Endquerschnitt ("Bogen- oder Sprengwerkwirkung") und Schubübertragung durch die Bügel ("Fachwerkwirkung") (Fig. 11.2). Aufgrund der vorhandenen Symotrie braucht dabei im Prinzip jeweils nur eine der beiden durch die Diagonale C-C' getrennten Scheibenhälfte betrachtet zu werden.

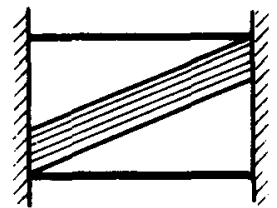

(a)

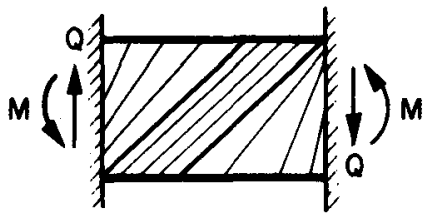

(b)

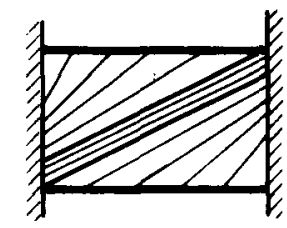

(c)

Fig. 11.2

(o) Direkte Schubübertrogung ("Bogen-, Sprengwerkwirkung")

(b) Schubübertragung durch Bügel ("Fachwerkwirkung")

(c) Gemischte Schubübertrogung 
Im vorliegenden Abschnitt wird vorerst angenommen, dass die Scheibe nur konzentrierte und verteilte Längsarmierung aber keine Bügelarmierung aufweist. Für diesen Fall ist die plastizitätstheoretische exakte Lösung bekannt. Die Querkraft kann dann nur über eine die Endquerschnitte direkt verbindende Druckdiagonale übertragen werden, wenn - wie in dieser Arbeit immer vorausgesetzt - die Betonzugfestigkeit null ist. Das entsprechende, fachwerkartige Spannungsfeld ist in Fig. 11.3 a dargestellt. Im Kollapszustand liegen die Betanspannungen im Bereich der Druckdiagonalen an der Betondruckfestigkeit, und für die in der Praxis üblichen Armierungsgehalte fliesst auch die gesamte Längsarmierung.

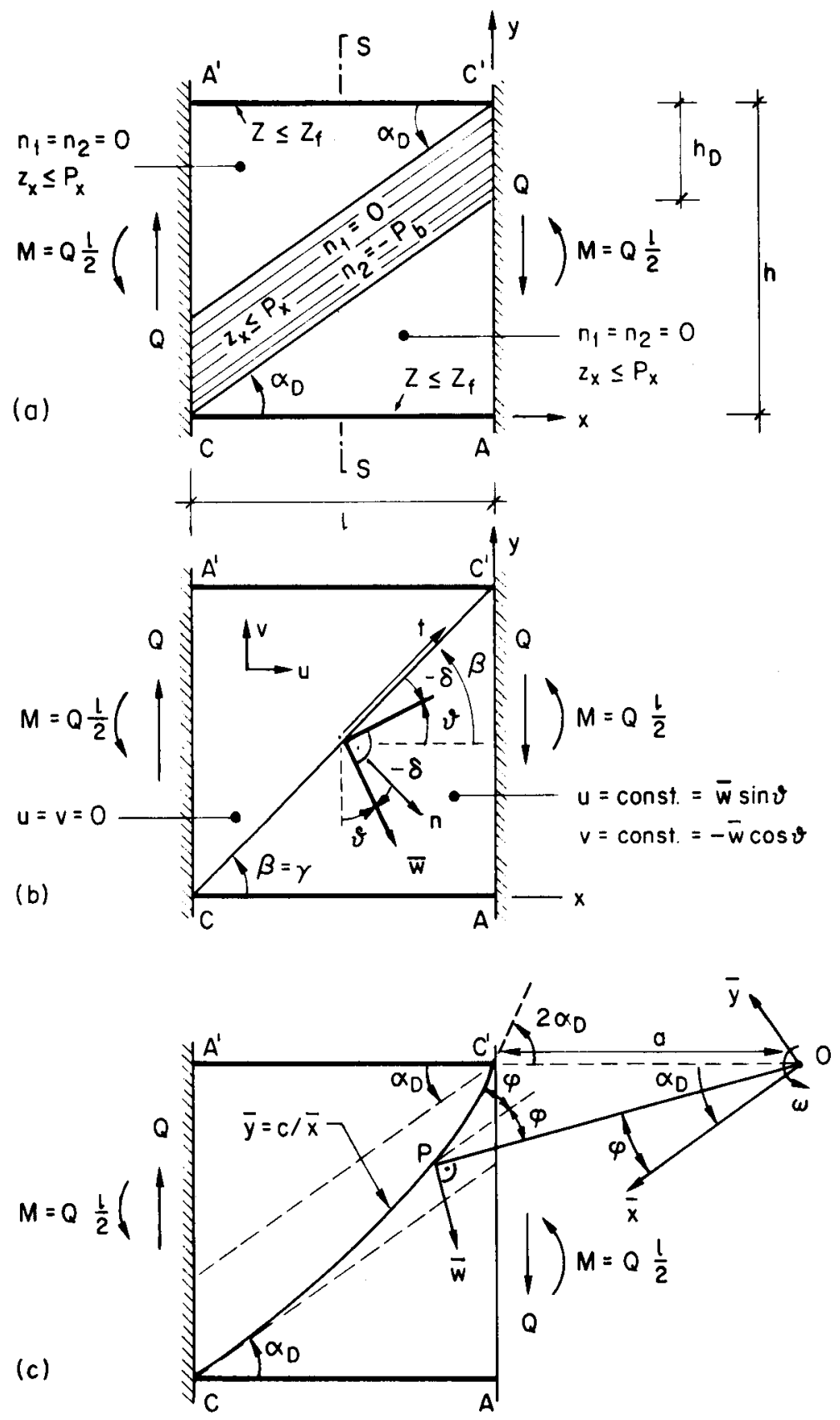

Fig. 11.3 Direkte Schubübertragung $\left(P_{y}=0\right)$ 
Mit den Bezeichnungen

$\Sigma z_{f}=2 Z_{f}+P_{x} h$

$h_{D}=h-1 \tan \alpha_{D}$

folgt aus den Gleichgewichtsbedingungen für den Querschnitt $S-S(M=0)$ sofort

$Q_{D}=\Sigma Z_{f} \tan \alpha_{D}=P_{b} \sin \alpha_{D} \cos \alpha_{D} h_{D}$

$\Sigma Z_{f}=P_{b} \cos ^{2} \alpha_{D} h_{D}$

Die aus (11.2) und (11.4) folgende implizite Bestimmungsgleichung für die Drucktrajektorienneigung $\alpha_{D}$ im Kollapszustand,

$\Sigma z_{f}=P_{b} \cos ^{2} \alpha_{D}\left(h-1 \tan \alpha_{D}\right)$

$\cos ^{2} \alpha_{D}-\frac{1}{h} \sin \alpha_{D} \cos \alpha_{D}=\sum Z_{f} /\left(P_{b} h\right)$

kann explizit formuliert werden, wenn man die Neigung $\gamma$ der Scheibendiagonalen $C-C$ ' einführt,

$\cot \gamma=1 / h$.

Es folgt dann

$1-\frac{2 \sum Z_{f}}{P_{b} h}=\sin ^{2} \alpha_{D}+\cos ^{2} \alpha_{D}-2 \cos ^{2} \alpha_{D}+2 \cot \gamma \cdot \sin \alpha_{D} \cos \alpha_{D}$,

$=\sin 2 \alpha_{D} \cot \gamma-\cos 2 \alpha_{D}$.

$=\sin \left(2 \alpha_{D}-\gamma\right) / \sin \gamma$,

und somit

$$
\left.\begin{array}{c}
\sin \left(2 \alpha_{D}-\gamma\right)=\left(1-\frac{2 \sum Z_{f}}{P_{b} h} \sin \gamma,\right. \\
\tan \gamma=h / 1 .
\end{array}\right\}
$$

Für die maximale Querkraft, die bei gegebener Betonfestigkeit übertragen werden kann, ergibt sich

$\frac{\partial Q_{D}}{\partial \alpha_{D}}=\frac{\partial}{\partial \alpha_{D}}\left(P_{b} \sin \alpha_{D} \cos \alpha_{D}\left(h-1 \tan \alpha_{D}\right)\right)=0$

$h \cos 2 \alpha_{D}-1 \sin 2 \alpha_{D}=0$

$\tan 2 \alpha_{D}=\tan \gamma, \quad 2 \alpha_{D}=\gamma$

$Q_{D}=\frac{1}{2} P_{b} h \tan \frac{\gamma}{2}$

und die für die Uebertragung dieser Querkraft minimal erforderliche Fliesskraft der Längsarmierung beträgt 
$\Sigma Z_{f}=Q_{D} \cot \alpha_{D}=\frac{1}{2} P_{b} h$

Bei grösserer Fliesskraft, $\Sigma Z_{f}>\frac{1}{2} P_{b} h$, kommt die Längsarmierung beim Kollaps offensichtlich nicht mehr ins Fliessen, und $Q_{\square}, \alpha_{D}$ sind durch (11.8) gegeben.

Es soll nun noch die Behauptung verifiziert werden, dass diese äusserst einfachen Spannungszustände die plastizitätstheoretisch exakte Traglast für die bügellose Scheibe liefern. Einen möglichen, kinematisch zulässigen Kollapsmechanismus zeigt Fig. 11.3 b: Die untere Scheibenhälfte $A C C^{\prime}$ bewegt sich starr und translatorisch mit der Geschwindigkeit $\bar{w}$ nach unten und löst sich längs der Diagonalen C-C' von der oberen ruhenden Hälfte; die Diagonale C-C' ist Unstetigkeitslinie des Geschwindigkeitsfeldes mit einem konstanten Geschwindigkeitssprung $\bar{w}$. Einziger freier Parameter des Mechanismus ist die Neigung o bzw. $\delta$ des Sprungvektors $\bar{w}$. Die Koordinatensysteme und Winkel sind in Fig. 11.3 b gleich eingeführt wie in den Figuren 3.4 und 3.5, wobei die Neigung $B$ der Unstetigkeitslinie mit der Neigung $\gamma$ der Diagonalen C-C' zusammenfällt. Die Beziehungen von Abschnitt 3.3 können somit unmittelbar übernommen werden.

Die Dissipationsleistung des Betons für das differentielle Element dt der Unstetigkeitslinie ergibt sich mit $(3.13) \mathrm{zu}$

$d L_{d}=P_{b} d\left(-\dot{\varepsilon}_{2}\right) d t=\frac{1}{2} P_{b}\left(\sqrt{w^{2}+q^{2}}-w\right) d t$,

wobei w, q gemäss Fig. 3.5 die Komponenten von $\bar{w}$ im $n$-t-Koordinatensystem sind. Mit

$\bar{w}=\sqrt{w^{2}+q^{2}}, \quad \cos \delta=w / \sqrt{w^{2}+q^{2}}$,

lässt sich (11.10) auch schreiben als

$d L_{d}=\frac{1}{2} P_{b} \bar{w}(1-\cos \delta) d t=\frac{1}{2} P_{b} \bar{w}(1-\cos (\beta-\vartheta)) d t$.

Die gesamte Dissipationsleistung und die äussere Leistung betragen somit

$L_{d}=\Sigma Z_{f} \bar{w} \sin \vartheta+\frac{1}{2} P_{b} \bar{w}(1-\cos (\beta-\vartheta)) h / \sin \beta$,

$L_{a}=Q_{D} \bar{w} \cos \vartheta$,

wobei $\vartheta \geq 0$ vorausgesetzt wird. Der Mechanismus ist instabil für $L_{a} \geq L_{d}$, also für Lasten grösser oder gleich

$Q_{D}=\Sigma Z_{f} \tan \vartheta+\frac{1}{2} P_{b} h \frac{1-\cos (\beta-\vartheta)}{\sin \beta \cos \vartheta}$,

$Q_{D}=\Sigma Z_{f} \tan \vartheta+\frac{1}{2} P_{b} h\left\{\frac{1}{\sin \beta \cos \vartheta}-(\tan \vartheta+\cot \beta)\right\}$.

Der obere Grenzwert (11.16) für die Traglast wird minimal für

$\frac{\partial Q_{D}}{\partial \vartheta}=\frac{1}{\cos ^{2} \vartheta}\left\{\Sigma Z_{f}+\frac{1}{2} P_{b} h\left(\frac{\sin \vartheta}{\sin \beta}-1\right)\right\}=0$,

$\sin \vartheta=\left(1-\frac{2 \Sigma Z_{f}}{P_{b} h}\right) \sin \beta$.

und lässt sich mit (11.17) ausdrücken durch 
$Q_{D}=\Sigma Z_{f} \tan \frac{\vartheta+\beta}{2}$.

Die Betondrucktrajektorien der mit diesem Mechanismus verträglichen Spennungszustände weisen auf der Diagonalen C-C' gemäss (3.18) die Neigung

$\alpha_{D}=\frac{1}{2}(v+\beta)=\frac{1}{2}(v+\gamma)$

auf.

Mit (11.19) wird aber sofort deutlich, dass der durch (11.17), (11.18) gegebene obere Grenzwert für die Traglast identisch ist mit dem unteren Grenzwert gemäss (11.7), (11.3). Der Gültigkeitsbereich von (11.17), (11.18): v $\geq 0, \Sigma Z_{f} \leq \frac{1}{2} P_{b} h$, deckt sich mit jenem für (11.7), (11.3), und damit ist für den Fall massgebender Längsarmierung die exakte Traglast gefunden.

Für

$v=0, \quad 2 \alpha_{D}=\beta=\gamma$,

folgt aus (11.15) der obere Grenzwert

$Q_{D}=\frac{1}{2} P_{b} h \frac{1-\cos \beta}{\sin \beta}=\frac{1}{2} P_{b} h \tan \frac{\beta}{2}, \beta=\gamma$.

Da dieser mit dem für $\Sigma Z_{f} \geq \frac{1}{2} P_{b} h$ gültigen unteren Grenzwert (11.8b) zusammenfällt, ist auch für den Fall nicht massgebender Längsarmierung die exakte Traglast gefunden.

Dass die Geschwindigkeitsfelder im Kollapszustand im allgemeinen nicht eindeutig sind, belegt Fig. 11.3 c. Sie zeigt für den Fall massgebender Längsarmierung $\left(\Sigma Z_{f}<\frac{1}{2} P_{b} h\right)$ einen weiteren mit dem Kollapsspannungszustand verträglichen Mechanismus. Die untere Scheibenhälfte löst sich nun entlang einer gekrümmten Unstetigkeitslinie von der oberen, ruhenden Hälfte und rotiert starr um ein Momentanzentrum 0 , das auf der Verlängerung des oberen Scheibenrandes liegen soll. Die Form der Unstetigkeitslinie ermittelt man am einfachsten in einem zur Druckdiagonalenneigung $\alpha_{D}$ parallelen Koordinatensystem $\bar{x}, \bar{y}$ mit Ursprung in 0 .

Da die Drucktrajektorienrichtung den kleineren Winkel zwischen der Tangente an die Unstetigkeitslinie und der Normalen zum Geschwindigkeitssprung $\bar{w}$ halbiert (Fig. $11.3 \mathrm{c}$ ), weist im $\bar{x}-\bar{y}$-System die Unstetigkeitslinie in jedem Punkt $P$ die betragsmässig gleiche, aber negative Neigung auf wie der Ortsvektor OP. Es gilt somit

$$
\begin{aligned}
-\bar{y}, & =\bar{y} / \bar{x}, \\
\bar{y} & =c / \bar{x},
\end{aligned}
$$

und die Unstetigkeitslinie ist eine Hyperbel. Aus den zwei Bedingungen, dass sie durch die beiden Punkte $C$ und $C^{\prime}$ gehen muss, bestimmen sich die Konstante 0 und der Abstand a des Momentanzentrums 0 von der Scheibe. Mit der Wahl von 0 auf der Verlängerung des obern Scheibenrandes wurde die Neigung der Unstetigkeitslinie in $C^{\prime} z u 2 \alpha_{0}$ (Fig. $11.3 \mathrm{c}$ ) vorgewählt und dieser Mechanismus ist somit für $\alpha_{D} \leq 45^{\circ}$ kinematisch zulässig. Diese Wahl des Momentanzentrums ist nicht zwingend. Alle anderen Mechanismen, die mit dem Kollapsspannungszustand verträglich sind, und deren Momentanzentrum rechts der Scheibe liegt, weisen jedoch Unstetigkeitslinien auf, die zwischen jenen gemäss Fig. 11.3 b und 11.3 c verlaufen. 
Nähert man sich dem Fall nicht massgebender Längsarmierung, $\Sigma Z_{f}+\frac{1}{2} P_{b} h$, so strebt die Neigung der Unstetigkeitslinie in C', $2 \alpha_{0}$, gegen die Neigung der Scheibendiagonalen C-C', das Momentanzentrum 0 wandert ins Unendliche, $a \rightarrow \infty$, die Unstetigkeitslinie degeneriert zur Geraden, und der Mechanismus geht über in den Mechanismus $v=0$ von Fig. 11.3 b. Der Mechanismus belegt die in Abschnitt 9.5 gemachte Feststellung, wonach gekrümmte Gleitlinien in Kanten, nicht aber in Flächen der Fliessfigur entsprechenden Fliessregimes mäglich sind.

Die Grenze zwischen dem Fall massgebender und dem Fall nicht massgebender Längsarmierung liegt auch hier wieder bei einem bezogenen, totalen Längsarmierungsgehalt von

$\bar{\mu}=\frac{\Sigma Z_{f}}{P_{b} h}=\frac{1}{2}$

Der Beton erreicht die Betondruckfestigkeit immer. Für unendliche Betonfestigkeit, $\bar{\mu}=0$, ergibt sich natürlich die Lösung des diskreten Fachwerkmodelles mit starrer Druckdiagonale, $Q_{D}=\Sigma Z_{f} h / l$.

Aus (10.17) folgt für diesen Fall $\vartheta=\beta$, der Geschwindigkeitssprung $\bar{w}$ (Fig. 10.3 b) steht senkrecht auf der Unstetigkeitslinie, und die Gleitlinie wird zum Kollapsriss. Entsprechend wird beim Mechanismus gemäss Fig. 10.3 c die gekrümmte Gleitlinie zum geraden Kollapsriss und das Momentanzentrum liegt dann in $C^{\prime}$. Nur in diesem rein theoretischen Fall $\bar{\mu}=0$ ist ein Mechanismus mit Momentanzentrum in $C^{\prime}$ und Kollapsriss $C^{\prime}-A$ mit dem Spannungszustand verträglich.

\subsection{Schubübertragung durch Bügel allein}

Die Schubübertragung erfolgt durch die Bügel allein, wenn die übertragene Querkraft kleiner oder gleich der totalen Fliesskraft aller Bügel ist. Um die Frage zu beantworten, ob Sprengwerkwirkung zu erwarten ist oder nicht, interessiert es deshalb, ob die Längsarmierung genügt, um eine Querkraft in der Grösse der totalen Bügelfliesskraft

$Q_{B}=P_{y} 1$

zu ت̈bertragen. Der in bezug auf die Beanspruchung der Längsarmierung günstigste Fächer (siehe Abschnitt 9.1) zur Einleitung von $Q_{B}$ in den Endquerschnitt ist in Fig. 11.4 ausgezogen dargestellt. Ist die Längsarmierung für diesen Fächer nicht ausgenützt, so wird ein flacherer Fächer möglich (Fig. 11.4, strichliert), es entsteht Raum für eine Druckdiagonale und es kann eine grösssere Querkraft als $Q_{B}$ übertragen werden. Genügt die Längsarmierung nicht, muss die Querkraft kleiner sein als $Q_{B}$. Die Drucktrajektorien verlaufen dann überall steiler als die Scheibendiagonale und die Scheibe wirkt wie ein Balken.

Aus dem Gleichgewicht der Scheibenhälfte ACC' folgt sofort, dass die totale Zugkraft der Längsarmierung auf der Diagonalen $C^{-C^{\prime}}$

$2 z_{C}+z_{x} h=0$

null ist, da entlang dieser keine Betonspannungen übertragen werden. Da der angenommene Betonspannungszustand allein im Gleichgewicht ist, ist die Beanspruchung der verteilten Armierung über die ganze Scheibe konstant. 


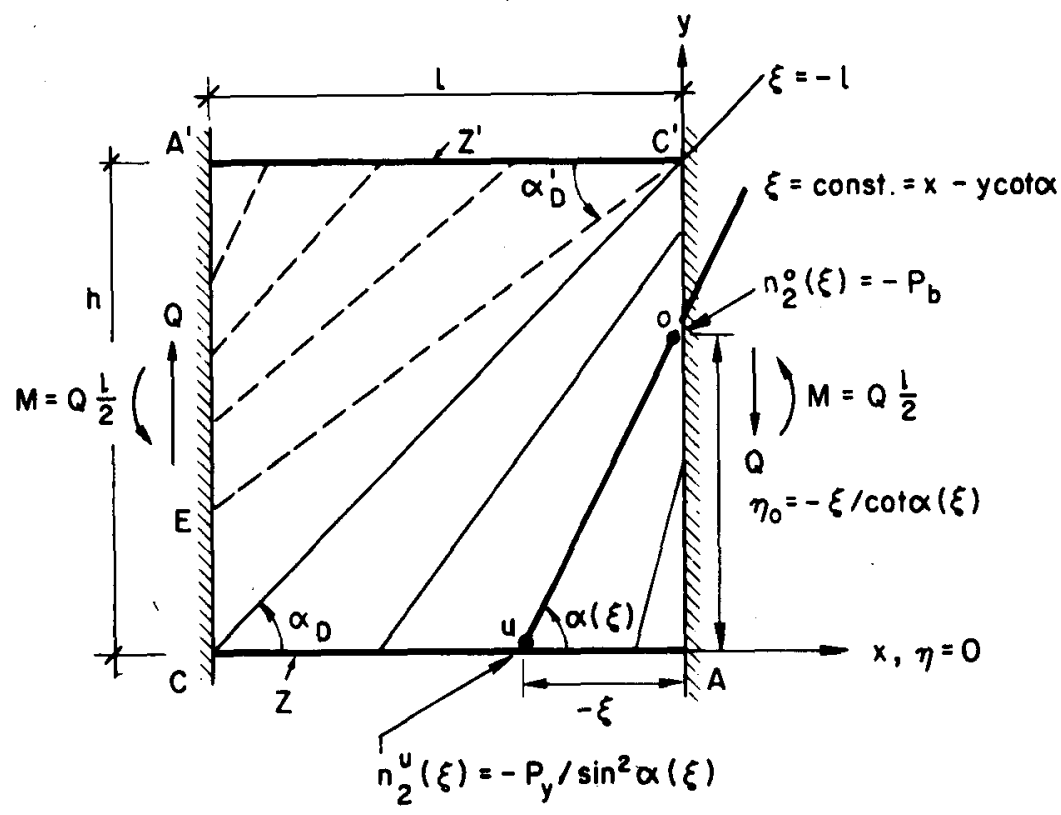

Fig. 11.4 Schubübertragung durch Bügel allein

Die Beanspruchung $Z_{A}$ der Hauptlängsarmierung im Punkt $A$ bestimmt man am einfachsten durch Integration der Schubspannungen längs des Scheibenrandes $A-C$

$Z_{A}=Z_{C}+Z_{B}$,

$Z_{B}=\int_{x=-1}^{x=0}\left(-N_{x y}\right) d x$

Aus der Randbedingung am Rand $A-C$

$N_{y}=P_{y}+n_{2} \sin ^{2} \alpha={ }^{\prime} 0$

folgt

$N_{x y}=n_{2} \sin \alpha \cos \alpha=-P_{y} \cot \alpha$

wobei $\alpha$ gemäss Abschnitt 9.1 Funktion von $\xi=x$ - ycota ist. Einsetzen von (11.27) in (11.25) ergibt mit $\xi(y=0) \Rightarrow x$

$Z_{B}=P_{y} 1 \frac{1}{I} \int_{\xi=-1}^{\xi=0} \cot \alpha(\xi) d \xi$

oder mit $\bar{\xi}=-\xi / 1$

$\left.\begin{array}{l}z_{B}=Q_{B} \cot \alpha_{m} \\ \cot \alpha_{m}=\int_{0}^{1} \cot \alpha(\bar{\xi}) d \bar{\xi}\end{array}\right\}$

Die Beanspruchung der Längsarmierung infolge $Q_{B}$ ist gemäss (11.29) umso kleiner, je schneller sich die Orucktrajektorien von C'nach A steiler stellen. Da der Spannungszustand stabil sein soll, darf die Aenderung der Trajektorienneigung jedoch nur so gross sein, dass am Scheibenrand $x=0$ gerade die Betondruckfestigkeit erreicht wird. Der in bezug auf die Beanspruchung 
der Längsarmierung günstigste, fächerartige Betonspannungszustand genügt deshalb der Randbedingung

$n_{2}\left(x=0,0<y \leq 1 \tan \alpha_{0}\right)=-P_{b}$.

wobei diese nur bis in beliebige Nähe des Punktes $A(x=y=0)$, nicht aber in A selbst gelten soll.

Für das Verhältnis der Betonspaninungen $\mathrm{n}_{2}$ in den Punkten u und o einer Trajektorie $\xi=$ const. (Fig. 11.4) folgt mit (11.26) und $(11.30)$ aus (9.22)

$\frac{n_{2}^{u}(\xi)}{n_{2}^{o}(\xi)}=1+n_{0} k^{\prime}(\xi)=\frac{P_{y} / \sin ^{2} \alpha(\xi)}{P_{b}}$.

Mit

$n_{0}=n(x=0)=-\xi / k(\xi)$

$1 / \sin ^{2} \alpha=1+\cot ^{2} \alpha=1+k^{2}$,

$P_{y} / P_{b}=\bar{\mu}$,

lautet die für $\xi \neq 0$ gültige Differentialgleichung für $k(\xi)=\cot \alpha(\xi)$

$1-\xi \frac{k^{\prime}(\xi)}{k(\xi)}=\bar{\mu}\left(1+k^{2}(\xi)\right)$

$\frac{d \xi}{\xi}=\frac{d k}{(1-\bar{\mu}) k-\bar{\mu} k^{3}}$,

$2(1-\bar{\mu}) \frac{d \xi}{\xi}=-\frac{\left(-\frac{2}{k^{3}}\right) d k}{\frac{1}{k^{2}}-\frac{\bar{\mu}}{1-\bar{\mu}}}$.

Mit den Abkürzungen

$\left.\begin{array}{l}p=2(1-\bar{\mu}) \\ k_{k}^{2}=\cot ^{2} \alpha_{K}=\frac{1-\bar{\mu}}{\bar{\mu}},\end{array}\right\}$

und (11.28) ergibt die Integration von (11.33)

$\bar{\xi}^{p}=c\left(\frac{k_{k}^{2}}{k^{2}}-1\right)^{-1}$,

und durch Auflösen nach $k$ folgt

$\left\|k(\bar{\xi})=k_{k}\left(1+\frac{c}{\bar{\xi}^{p}}\right)^{-1 / 2}\right\|$ 
Aus der Randbedingung im Punkt $C^{\prime}$ (oder analog $E$ ),

$\bar{\xi}=1: \quad k=\cot \alpha=\cot \alpha_{D}=k_{D}$,

folgt schliesslich die Konstante $C \mathrm{zu}$

$\| c=\left(k_{K} / k_{D}\right)^{2}-1$

$\alpha_{K}$ ist jene flachste, kritische Drucktrajektorienneigung, für welche die Betondruckfestigkeit bei fliessender Bügelarmierung schon am freien Scheibenrand erreicht ist: $P_{y}=P_{b} \sin ^{2} \alpha_{K}, \alpha_{K} \leq 45^{\circ}$. Wird im Punkt $C$ die Anfangsneigung $\alpha_{D} z u \alpha_{D}=\alpha_{K}$ vorgeschrieben, so ist die Konstante $C$ null, und der Betonspannungszustand kann für fliessende Bügelarmierung nur konstant sein. Bei Anfangsneigungen $\alpha_{D}>\alpha_{k}$ wächst die Trajektorienneigung $\alpha$ für $\bar{\xi} \rightarrow 0$ umso schneller gegen $90^{\circ}$, je steiler die Anfangsneigung $\alpha_{D}$ im Verhältnis zur kritischen Neigung $\alpha_{k}$ ist. Dabei gilt die Lösung bis in eine beliebige Nähe $\bar{\xi}=\varepsilon$ des Punktes A, nicht aber in A selbst, wo nur noch die Randbedingung (11.26) gilt. Zwischen einer letzten, sehr nahe an $A$ gelegenen Trajektorie $\bar{\xi}=\varepsilon$ und $A$ kann man sich denn noch einen sehr kleinen, zentrischen Fächer vorstellen mit Zentrum im Berührungspunkt der letzten Trajektorie $\bar{\xi}=\varepsilon$ mit der Hüllkurve.

Für unendich grosse Betonfestigkeit, $\bar{\mu}=0, \alpha_{K}=0$, geht die Differentialgleichung (11.33) und ihre Lösung (11.35), (11.36) über in die Beziehungen für den zentrischen Fächer mit Fächerzentrum am Rand $x=0$

$d \xi / \xi=d k / k, \quad k=k_{D} \bar{\xi}$.

Der Mittelwert $\cot \alpha_{m}$ und die Zugkraft $z_{B}$ betragen in diesem Fall

$\cot \alpha_{m}=\frac{1}{2} k_{D}=\frac{1}{2} \frac{1}{h}, \quad z_{B}=\frac{1}{h} Q_{B} \frac{1}{2}=\frac{1}{h} M(x=0)$

und entsprechen jenen Werten, die sich aus Gleichgewichtsbetrachtungen an von C' ausstrahlenden, diskreten, starren Fachwerkdiagonalen oder Rissen ergeben [44]. Mit diesem Vorgehen erhält man wohl einen guten Ueberblick über die generelle Tragwirkung und die Zugkraftvariation in der Längsarmierung. Offen bleibt aber die für die Ermittlung unterer Grenzwerte der Traglast wesentliche Frage, wie stark durch den Querkrafteinfluss der Hebelarm der inneren Kräfte im Einspannquerschnitt bei endlicher Betondruckfestigkeit reduziert wird.

Wie insbesondere auch der am Ende von Abschnitt 11.2 behandelte Grenzfall $P_{b}=\infty$ deutlich macht, entspricht die Ermittlung der Traglast einer Scheibe an Fachwerkmodellen mit diskreten, starren Druckdiagonalen der Mechanismusmethode mit jener Klasse von Geschwindigkeitsfeldern. die mit dem Fliessregime I verträglich sind. Diese liefert im Bereich von Einspannquerschnitten und Einzellasten immer die reine Biegetraglast.

Bisher wurde natürlich stillschweigend vorausgesetzt, dass cot $\alpha_{k}>1 / h$ ist. Ist die Bügelarmierung im Verhältnis zur Betonfestigkeit so stark bzw. die Scheibe so lang, dass dies nicht mehr zutrifft, so muss der Kollaps für $Q \leq Q_{B}$ erfolgen. Die Schubübertragung erfolgt über die Bügel allein unabhängig davon, ob zusätzlich zur Bügelarmierung der Beton im Feld oder die Längsarmierung im Einspannquerschnitt massgebend wird.

Für die Scheibe gemäss Fig. 11.1 ist cota $\gg 1 /$. Wie die im folgenden Abschnitt zusammenge- 
stellten Resultate zeigen, ist für den in Fig. 11.4 ausgezogen dargestellten Fächer die Längsarmierung unter der Last $Q=Q_{B}$ bei weitem nicht ausgenützt. Dies lässt sich übrigens schon mit einem einfachen, zentrierten Fächer nachweisen, dessen Fächerzentrum aus der Bedingung bestimmt wird, dass der Beton die Druckfestigkeit in den Punkten $c$ erreicht. Aus der Sicht stabiler, statisch zulässiger Spannungszustände - exakte Läsungen sind mit Ausnahme jener fälle, bei welchen die Längsarmierung nicht massgebend wird oder keine Bügelarmierung vorhanden ist, noch nicht bekannt - ist also eine zusätzliche Sprengwerkwirkung zu erwarten.

\subsection{Gemischte Schubübertragung}

Ist $\cot \alpha_{K}>1 / h$ und die Längsarmierung sterk genug, dass die totale Bügelfliesskraft $Q_{B}$ auch über einen flacheren, in Fig. 11.4 strichliert dargestellten Fächer in den Endquerschnitt übertragen werden kann, so lassen sich die Spannungszustände von Fig. 11.3 a und Fig. 11.4 kombinieren, wie es Fig. 11.5 a zeigt.

Die Gleichungen, die die übertragene Querkraft, die Druckdiagonalenneigung $\alpha_{0}$ und die Beanspruchung der Längsarmierung verknüpfen, lassen sich im wesentlichen sofort den Abschnitten 11.2 und 11.3 entnehmen.

$Q=Q_{B}+Q_{D} \quad$,

$Q_{B}=P_{y} I$

$Q_{D}=P_{b} \sin \alpha_{D} \cos \alpha_{D}\left(h-1 \tan \alpha_{D}\right)$

$Z_{A}=z_{B}+Z_{C} \quad$,

$z_{B}=Q_{B} \cot \alpha_{m}$,

$z_{C}=\frac{1}{2}\left(Q_{D} \cot \alpha_{D}-z_{X} h\right)$.

Dabei ist cotam mit (11.35), (11.36) und der zweiten Gleichung (11.29) ebenfalls Funktion von $\alpha_{D}$. Die Auswertung des Integrals in (11.29) erfolgt dabei am einfachsten mit den Quadraturformeln von Gauss, wobei mit zwei Integrationspunkten schon genügende Genauigkeit erreicht ist.

Wird die Längsarmierung für den höchsten, mit diesem Spannungsfeld erreichbaren, unteren Grenzwert der Traglast massgebend,

$z_{A}=z_{f}, \quad z_{x}=P_{x}$

so besitzt man mit (11.39) bis (11.41) ein Gleichungssystem für $\alpha_{D}$, das sich sofort - ausgehend von $\tan \alpha_{D}=h / l$ - durch Probieren lösen lässt.

Die erfolglose Suche nach einem kinematisch zulässigen Mechanismus, der mit dem untersuchten Spannungszustand bei massgebender Längsarmierung verträglich ist, lässt vermuten, dass in der exakten Lösung im Bereich der Ecken C Unstetigkeiten des Spannungsfeldes auftreten, bei welchen der Spannungszustand von einem Fliessregime in ein anderes springt. 


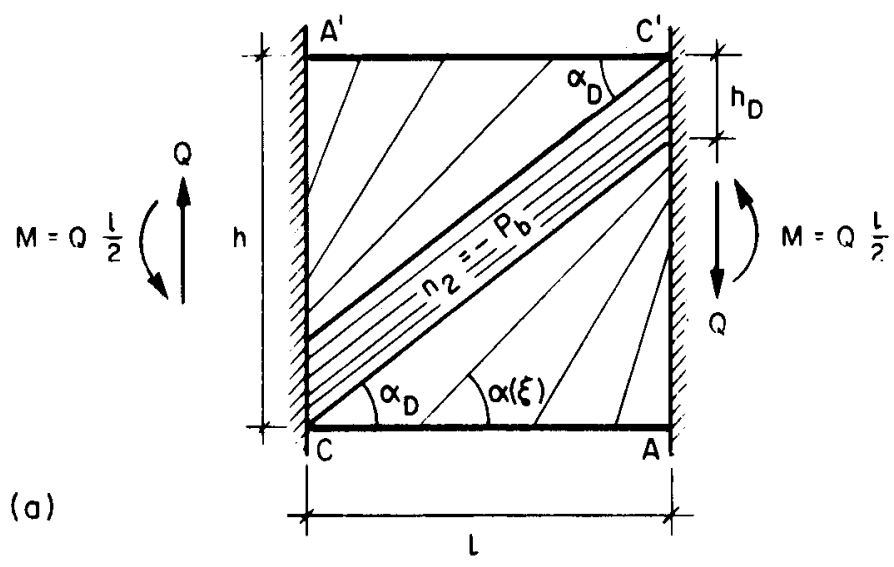

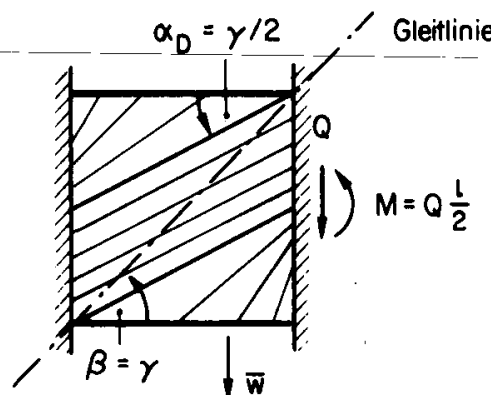

$\alpha_{k}<\gamma^{\prime 2}$

(b)

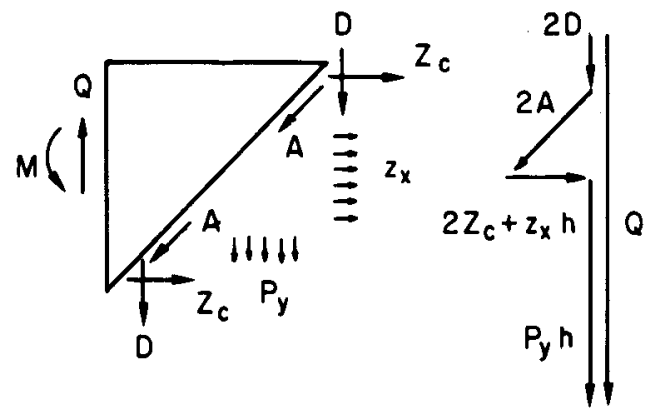

(d)

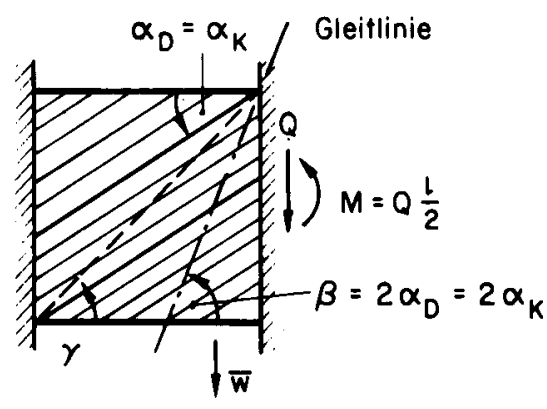

$\alpha_{k} \geq \gamma / 2$

(c)

Fig. 11.5 Gemischte Schubübertragung

Im Falle nicht massgebender Längsarmierung sind wieder die exakten kollapslasten bekannt. Es lassen sich zwei Fälle unterscheiden. Dabei soll wieder mit $\gamma$ die Neigung der Scheibendiagonalen $C-C$ ' und mit $\beta$ die Neigung 'der Gleitlinie des Kollapsmechanismus bezeichnet werden, längs welcher sich die untere Scheibenhälfte von der oberen löst und sich senkrecht nach unten bewegt. Ist $\alpha_{K}<\gamma / 2$, so gilt im Kollapszustand $\alpha_{D}=\gamma / 2$, die Gleitlinie fällt mit der Scheibendiagonalen zusammen, $\beta=\gamma$,und die Traglast ist durch (11.38), (11.39a) und (11.8b) gegeben (Fig. 11.5 b). Ist andererseits $45^{\circ} \geq \alpha_{K} \geq \gamma / 2$, so ist der Kollapszustand für $\alpha_{D}=\alpha_{K}$ erreicht. Der Betonspannungszustand ist über die gesamte Scheibe konstant und liegt an der Druckfestigkeit. Die Gleitlinierineigung ist nun mit $\beta=2 \alpha_{D}=2 \alpha_{K}$ steiler als die Diagonalenneigung. Spannungszustand und Kollapsméchanismus entsprechen offensichtlich den in Abschnitt 7 untersuchten, und die Traglast ist durch (7.37) gegeben. 
Beim wandartigen Träger gemäss Fig. 11.1 ist die Längsarmierung sowohl theoretisch als auch experimentell massgebend. Die nachstehende Tabelle zeigt die Ergebnisse der Auswertung der Gleichungen (11.38) bis (11.41) zusammen mit den experimentellen Resultaten [44] und dem Rechenergebnis von Cervenka [46]. Die theoretischen unteren Grenzwerte für die Traglast sind sowohl für den Fall mitwirkender $(k=1)$ als auch für den Fall nicht mitwirkender $(k=2 / 3)$ Betonüberdeckung der Bügel ermittelt.

\begin{tabular}{|l|c|c|c|c|}
\hline & $\begin{array}{c}\text { Experiment } \\
{[44]}\end{array}$ & $\begin{array}{c}\text { Theorie } \\
k=1\end{array}$ & $\begin{array}{c}\text { Theorie } \\
k=2 / 3\end{array}$ & $\begin{array}{c}\text { Cervenka } \\
{[46]}\end{array}$ \\
\hline & Kips & Kips & Kips & Kips \\
$Q$ & 174.5 & 182 & 168 & 200 \\
$Z_{A}$ & 105 & 105 & 105 & - \\
$Z_{C}$ & 250 & 17 & 9 & - \\
\hline
\end{tabular}

Der Vergleich der experimentellen und theoretischen Resultate zeigt, dass sich die Tragfähigkeit auch bei Scheibenproblemen mit relativ einfachen, aber wirklichkeitsnahen Spannungsfeldern befriedigend abschätzen lässt. Voraussetzung für die Anwendbarkeit der Plastizitätstheorie ist allerdings, dass eine genügend starke, verteilte Armierung vorhanden ist, so dass die Betonzugspannungen beim Bruch keine Rolle spielen. Dies ist zum Beispiel bei den von Leonhardt und Walther [42] geprüften, von oben belasteten, wandartigen Trägern nicht der Fall, bei denen die verteilte Armierung kaum die Betonzugfestigkeit abdeckte.

In Fig. 11.5 ist noch das Gleichgewicht entlang der Hauptdiagonalen nach üblicher Auffassung des Versuchspraktikers (Fig. 11.5 d) und nach dem plastizitätstheoretischen Ansatz dargestellt (Fig. 11.5 e). Die erste Betrachtungsweise erklärt die Differenz zwischen beobachteter Querkraft und totaler Bügelfliesskraft durch "Aggregat Interlock" A und "Dowel Action" $D$ am Riss entlang der hauptdiagonalen, die zweite durch eine Umlagerung der Betondrucktrajektorienrichtung. Letztere bedeutet allerdings nicht, dass "Aggregat Interlock" und "Dowel Action" vernachlässigt werden. Ihre Wirksamkeit in den ersten sich bildenden Rissen, die natürlich nicht in Richtung der theoretischen Drucktrajektorien im Kollapszustand verlaufen, ist im Gegenteil Voraussetzung dafür, dass die angenommenen Spannungsumlagerungen stattfinden können. Dass solche Umlagerungen stattfinden, konnte z.B. in den Torsionsversuchen [34, 35, 36] sehr schön beobachtet werden, bei denen ein erstes Rissebild durch ein zweites mit deutlich anderer Neigung überlagert wurde. 
12.1 Schlusswort

Bereits vor mehr als einem halben Jahrhundert ist von Ritter und Mörsch zur Erklärung der Tragwirkung von gerissenen, schubbeanspruchten Stahlbetonbalken das Fachwerkmodell eingeführt worden. In den experimentellen und theoretischen Arbeiten von Grob, Lampert, Lüchinger, Thürlimann [18-23, 34-36] wurde gezeigt, dass mit dem Fachwerkmodell der Bruchwiderstand unterarmierter Stahlbetonbalken unter Torsion, Biegung und Querkraft gut erfasst werden kann, wenn die Neigung der Druckdiagonalen bzw. des Druckfeldes aus der Bedingung bestimmt wird, dass Längs- und Bügelarmierung fliessen. Das Modell bildet deshalb heute in verschiedenen Stahlbetonnormen die rationale Grundlage für die Bruchbemessung der Armierung [25, 29,30$].$

Ein wesentlicher Beitrag der vorliegenden Arbeit besteht darin, dass für Stahlbetonbalken mit dünnwandigem, geschlossenem Querschnitt unter Torsion und Biegung mit dem Spannungszustand des Fachwerkmodells [18, 19, 22] verträgliche Kollapsmechanismen abgeleitet werden konnten. Der Spannungszustand gemäss "Fachwerkmodell" und die neuen dreidimensionalen Mechanismen bilden zusammen die plastizitätstheoretisch exakte Lösung innerhalb einer Theorie, die die Schubwände als Membranen mit der Fliessbedingung $(2.7$ I) idealisiert. Dem praktischen Ingenieur können die Mechanismen bei Spezialproblemen Hinweise für die Dimensionierung geben.

Sobald Querkräfte vorhanden sind, liegen die massgebenden Querschnitte wegen des Momentengradienten meist auch bei Versuchsbalken bei Lasteinleitungsstellen. Auflagern oder Verstärkungen, wo die Betonspannungsfelder im Steg stark variieren, und die Beanspruchung der Armierung infolge Querkraft vom Spannungskonzentrationsvermögen im Beton und damit von der Betondruckfestigkeit abhängt. Entsprechendes gilt natürlich auch bei wölbtorsion. Bei kurzen oder wandartigen Trägern tritt zudem zusätzlich Sprengwerkwirkung auf.

Die weiteren Beiträge der Arbeit befassen sich deshalb mit der Ueberprüfung der Frage, ob die Plastizitätstheorie auch bei massgebender Betondruckfestigkeit anwendbar ist, und mit der Ableitung von Beziehungen für die allgemeinen Spannungs- und Geschwindigkeitsfelder der plastifizierten Stahlbetonscheibe. Die Ergebnisse des Studiums der oberen Schubspannungsgrenze und der Fallstudie an einem wandartigen Träger lassen vermuten, dass mittels der Plastizitätstheorie bei der Erfassung des Querkrafteinflusses auf die Traglast von Balken und wandartigen Trägern noch Fortschritte zu erzielen sind.

\subsection{Zusammenfassung}

Die vorliegende Arbeit behandelt theoretische Grundlagen der plastischen Berechnung von Stahlbetonscheiben und schubbeanspruchten, dünnwandigen Stahlbetonbalken. Die Studie basiert auf der klassischen Plastizitätstheorie, insbesondere auf den Grenzwertsätzen und der Theorie des plastischen Potentials.

Unter Vernachlässigung der Betonzugfestigkeit werden fliessbedingungen für orthogonal und nichtorthogonal armierte Stahlbetonscheiben hergeleitet. Dabei wird sowohl der idealisierte Fall gleicher als auch der wirklichkeitsnahere fall unterschiedlicher ein- und zweiaxialer Betondruckfestigkeit behandelt. Die Statik und Kinematik der einzelnen Fliessregimes wird diskutiert. 
Das Konzept des Fachwerkmodelles mit variabler Diagonalenneigung, das in [18] zur Erfassung des Bruchwiderstandes von Stahlbetonbalken unter Torsion und Biegung verwendet wurde, wird diskutiert. Es wird darauf hingewiesen, dass das Fachwerk wohl sehr anschaulich des Kräftespiel, nicht aber die Kinematik der plastifizierten Stahlbetonscheibe zu erfassen vermag. Querschnittsinteraktionsbeziehungen werden in der plastischen Balkentheorie normalerweise über die Definition verallgemeinerter Spannungen und Verformungen aus den Fliessbedingungen des Kontinuums abgeleitet. Es wird gezeigt, dass die statischen Annahmen des Fachwerkmodells obigen Scheibenfliessbedingungen und den üblichen vereinfachenden Annahmen der Balkentheorie entsprechen. Implizit wird ein Satz von verallgemeinerten Spannungen und Verformungen verwendet, der die freie Verwölbbarkeit des Querschnittes voraussetzt. Unter dieser Annahme können die mit dem Fachwerkmodell gewonnenen Interaktionsbeziehungen auch sofort über die Dissipationsleistung des Querschnittes erhalten werden.

Für Balken mit dünnwandigem, geschlossenem Querschnitt unter konstantem Torsions- und Biegemoment werden räumliche Kollapsmechanismen mit diskontinuierlichem Geschwindigkeitsfeld angegeben, die mit dem stabilen, statisch zulässigen Spannungszustand gemäss Fachwerkmodell

$[18,20,22]$ - insbesondere mit dem kanstanten St. Venant'schen Schubfluss - verträglich sind, solange nur die Membranwirkung der Schubwände in Rechnung gestellt wird. Die mit dem Fachwerkmodell gewonnenen Interaktionsbeziehungen liefern die exakte Traglast, solange sich diese Mechanismen unbehindert ausbilden können. Eine behinderte Längsausdehnung dieser Mechanismen führt zu nicht konstantem Schubfluss und damit zu einem gemischten Torsionsproblem. Der Nachweis, dass bei viereckigen Vollquerschnitten der Kernbeton keinen Beitrag zur Dissipationsleistung des Mechanismus leistet, bestätigt die Versuchsresultate, wonach gleich armierte Voll- und Hohlquerschnitte den gleichen Torsionswiderstand aufweisen.

In [26] und in der vorliegenden Arbeit wird gezeigt, dass die Plastizitätstheorie für Balken mit dünnem Steg unter Biegung und Querkraft auch dann vernünftige Resultate liefert, wenn der Schubbruch durch Versagen des Stegbetons erfolgt: Die theoretische Abhängigkeit der oberen Schubspannungsgrenze von Betondruckfestigkeit und Bügelfliesskraft wird in ihrem generellen Verlauf von den Versuchsresultaten bestätigt.

Die in den verschiedenen Fliessregimes möglichen allgemeinen Spannungs- und Geschwindigkeitsfelder der plastifizierten Stahlbetonscheibe werden untersucht. Als Anwendungsbeispiele für die erhaltenen Beziehungen werden Schubprobleme in Balken mit dünnen Stegen bei variabler Querkraft oder Balkenhöhe und Lasteinleitungsprobleme studiert und Schlussfolgerungen für die Bemessung gezogen. Die Arbeit schliesst mit einer Fallstudie an einem wandartigen Träger. Mit relativ einfachen, aber wirklichkeitsnahen Spannungsfeldern lässt sich eine brauchbare Abschätzung der Traglast und der sogenannten Sprengwerkwirkung gewinnen.

12.3 Abstract

The present study deals with theoretical fundamentals of plastic analysis of reinforced concrete shear walls and thinwalled reinforced concrete beams in torsion and shear. It contributes towards a rational basis for ultimate strength design in reinforced concrete. The study is based on the theory of perfectly plastic solids and the fundamental theorems of limit analysis.

Yield criterions and flow rules for reinforced concrete shear walls are derived and discussed. Orthogonal and non-orthogonal reinforcement as well as equal and unequal uniaxial and biaxial concrete strength are considered. 
The truss model introduced in [18] to calculate the ultimate strength of reinforced concrete beams in torsion and bending, is discussed. The interaction formulas derived by the truss model are shown to be based on the yield criterion of shear walls and a set of generalized stresses and deformations allowing for the warping of the fully plastic cross-section. New three-dimensional collapse mechanisms are presented. Within the scope of a theory considering only membrane action the truss model solution is shown to be the exact solution for underreinforced long beams with thin-walled closed cross-section in uniform torsion and bending. Moreover the new mechanisms demonstrate that the core of solid cross-sections does not contribute to the torsional ultimate strength.

The theory of plasticity is applied to shear failures by web crushing. The present study shows, as well as [26], that the test results confirm the theoretically predicted relation between nominal shear strength, concrete compressive strength and yield forces of stirrups.

The general stress and velocity fields of the fully plastic reinforced concrete shear wall are studied. The relations obtained are used to investigate the shear problem in thin-walled beams with variable shear force or beam depth and the vicinity of points of load application. The work ends with a case study for a reinforced concrete shear wall. By means of simple but realistic stress fields the collapse load and the effect of arch action may be estimated satisfactorily.

\subsection{Resumé}

La présente étude contribue à la théorie de l'analyse plastique des panneaux en beton armé et des poutres à parois minces en béton armé soumises à la torsion et à l'effort tranchant. L'étude est basée sur la théorie de la plasticité et sur les théorèmes fondamentaux de l'analyse limite.

Des critères de plasticité pour les panneaux en béton armé et les lois d'ecoulement plastique correspondantes sont stablies et discutés.

Le modèle de treillis introduit dans l'étude [18] pour le calcul à l'état-limite ultime des poutres soumises à la torsion et à la flexion uniforme est discute. On montre que les relations d'interaction établies à partir du modêle de treillis sont basées sur ces critères de plasticité et sur un ensemble de contraintes et de deformations géneralisées permettant le gauchissement de la section plastifiée. Des nouveaux mécanismes de ruine tri-dimensionels sont développés, démontrants que le modèle de treillis fournit la charge limite exacte pour des poutres-caissons longues à parois minces idéalisées comme membranes.

La théorie de la plasticite est appliquée au calcul à l'état-limite ultime des âmes de poutres minces soumises à.l'effort tranchant et détruites par écrasement du béton en compression. La présente étude, de même que [26], montre que les résultats d'essais confirment la rélation théorique entre la résistance ultime à l'effort tranchant et la résistance ultime du béton et de l'armature transversale.

Les champs de contraintes et de vitesses généraux des panneaux en béton armé complètement plastiques sont discutés. Les résultats obtenus servent à étudier l'influence d'un effort tranchant variable dans les poutres à âme mince de hauteur constante ou variable et la proximité des points d'application des charges. Finalement, un panneau en béton armé testé par Paulay [44] est ftudie. A l'aide de champs de contraintes simples, mais réalistes, une limite inférieure satisfaisante pour la résistance ultime et l'effet d'arc est determinee. 


\section{Grundlagen}

[1] Prager, William: Probleme der Plastizitätstheorie. Birkhäuser Verlag Basel und Stuttgart, 1955.

[11] D.C. Drucker, The Effect of Shear on the Plastic Bending of Beams, Journal of Applied Mechanics, vol. 23, Trans. ASME, vol. 78, 1956, p. 509.

[12] P.G. Hodge, Jr.: Interaction Curves for Shear and Bending of Plastic Beams, Journal of Applied Mechanics, vol. 24, Trans. ASME, vol. 79, 1957, p. 453.

[14] Stenbuch, K.: Technische Modelle biologischer Vorgänge. In: Probleme der Kybernetik. Herausgeber H.v. Diffurth. Fischer Taschenbuch Verlag, November 1971.

\section{Berechnung von Stahlbetontragwerken}

[15] Nielsen, M.P.: Yield Conditions for Reinforced Concrete Shells in the Membrane State, Reprint from Non-Classical Shell-Problems, Proc. IASS Symposium, Warswa, Sept. 2.-5. 1963 .

[16] Baumann, Th.: Tragwirkung orthogonaler Bewehrungsnetze beliebiger Richtung in Flächentragwerken aus Stahlbeton, Heft 217, Deutscher Ausschuss für Stahlbeton, 1972 .

[17] Gvozdev, A.A., Lessig N.N., Rulle, L.K.: Research on Reinforced Concrete Beams Under Combined Bending and Torsion in the Soviet Union. In Torsion of Structural Concrete, Journal American Concrete Institute, April 1968, p. 310. Publication SP-18.

[18] Lampert, P.: Bruchwiderstand von Stahlbetonbalken unter Torsion und Biegung, Bericht Nr. 26, Institut für Baustatik ETH Zürich, Birkhäuser Verlag Basel und Stuttgart, 1970 .

[19] Lampert, P.: Torsion und Biegung von Stahlbetonbalken. Sonderdruck aus SBZ, 88. Jahrgang, Heft 5. Bericht Nr. 27, Institut für Baustatik, ETH Zürich, Birkhäuser Verlag Basel und Stuttgart, 1970. Beams in Torsion and Bending. Sonderdruck aus IVBH-Abhandlungen, Band $31 / I, 1972$. Bericht Nr. 42 Institut für Baustatik, ETH Zürich, Birkhäuser Verlag Basel und Stuttgart, 1972. 
Grob, J.: Traglast von Stäben mit dünnwandigen offenen Querschnitten. Bericht Nr. 56, Institut für Baustatik und Konstruktion, ETH Zürich, Birkhäuser Verlag Basel und Stuttgart, 1975.

Thürlimann, B., Grob, J., Lüchinger, P.: Torsion, Biegung und Schub in Stahlbetonträgern, Institut für Baustatik und Konstruktion, ETH Zürich. Autographie zu Fortbildungskurs für Bauingenieure aus der Praxis, 1975.

Lüchinger, P.: Bruchwiderstand von Kastenträgern aus Stahlbeton unter Torsion, Biegung und Querkraft. Institut für Baustatik und Konstruktion, ETH Zürich, Bericht Nr. 69, Birkhäuser Verlag Basel und Stuttgart, 1977.

Müller, P.: Failure Mechanisms for Reinforced Concrete Beams in Torsion and Bending. Institut für Baustatik und Konstruktion, ETH Zürich, Bericht Nr. 65, Sonderdruck aus IVBH-Abhandlungen. Band 36-II, Zürich 1976. Birkhäuser Verlag Basel und Stuttgart, 1976.

Grob, J., Thürlimann, B.: Ultimate Strength and Design of Reinforced Concrete Beams under Bending and Shear, Institut für Baustatik und Konstruktion, ETH Zürich, Bericht Nr. 63, Sonderdruck aus IVBH-Abhandlungen Band 36-II, Zürich 1976. Birkhäuser Verlag Basel und Stuttgart, 1976.

Braestrup, M.W.: Plastic Analysis of Shear in Reinforced Concrete, Magazine of Concrete Research, Vol. 26, No. 89, Dec. 1974.

Elfgren, L., Karlsson, I., Losberg, A.: Nodal Forces in the Analysis of the Ultimate Torsional Moment for Rectangular Beams. Magazine of Concrete Research, Vol. 26, No. 86, March 1974, pp. 21-28.

Norm SIA 162, Norm für die Berechnung, Konstruktion und Ausführung von Bauwerken aus Beton, Stahlbeton und Spannbeton, Schweiz. Ingenieur- und Architekten-Verein (SIA), Zürich, 1968.

Richtlinie 34 zu Norm SIA 162, Bruchwiderstand und Bemessung von Stahlbeton- und Spannbetontragwerken, Schweiz. Ingenieur- und Architekten-Verein, Zürich, 1976.

Comité Euro-International du Béton: Code modèle pour les structures en béton, Système international de réglementation technique unifiée des structures, vol. II, Bulletin d'Information no 117 , Paris, décembre 1976.

\section{Versuchsberichte}

[32] Kupfer, H., Hilsdorf, H.K., Rüsch, H.: Behavior of Conerete Under Biaxial Stresses, ACI-Journal, August 1969.

[33] Peter, J.: Zur Bewehrung von Scheiben und Schalen für Hauptspannungen schiefwinklig zur Bewehrungsrichtung. Dissertation Technische Hochschule Stuttgart, 1964 .

Lampert, P., Thürlimann, B.: Torsionsversuche an Stahlbetonbalken, Institut für Baustatik, ETH Zürich, Bericht Nr. 6506-2, Juni 1968, Birkhäuser Verlag Basel und Stuttgart.

Lampert, P.; Thürlimann, B.: Torsions-Biege-Versuche an Stahlbetonbalken, Institut für Baustatik, ETH Zürich, Bericht Nr. 6506-3, Januar 1969, Birkhäuser Verlag Basel und Stuttgart.

Lampert, P., Lüchinger P., Thürlimann, B.: Torsionsversuche an Stahl- und Spannbetonbalken, Institut für Baustatik. ETH Zürich, Bericht Nr. 6506-4, Feb. 1971. Vergriffen.

Caflisch, R., Thürlimann, B.: Schubversuche an teilweise vorgespannten Betonbalken. Institut für Baustatik, ETH Zürich, Bericht $\mathrm{Nr}$. 6504-2, 1970. Birkhäuser Verlag Basel und Stuttgart.

Caflisch, R., Krauss, R., Thürlimann, B.: Biege- und Schubversuche an teilweise vorgespannten Betonbalken, Serie C. Institut für Baustatik, ETH Zürich, Bericht Nr. 6504-3, 1971, Birkhäuser Verlag Basel und Stuttgart. 
[39] Krauss, R., Heimgartner, E., Bachmann, H.: Versuche über den Einfluss geneigter Spannkabel in teilweise vorgespannten Betonbalken. Institut für Baustatik, ETH Zürich, Bericht Nr. 6504-6, 1973. Birkhäuser Verlag Basel und Stuttgart.

[40] Leonhardt, F., Walther, R.: Schubversuche an einfeldrigen Stahlbetonbalken mit und ohne Schubbewehrung. Deutscher Ausschuss für Stahlbeton, Heft 151, Verlag Wilhelm Ernst und Sohn, Berlin, 1962.

[41] Leonhardt, F., Koch, R., Rostasy, F.S.: Schubversuche an Spannbetonträgern. Deutscher Ausschuss für Stahlbeton, Heft 227, Verlag Wilhelm Ernst und Sohn, Berlin 1973.

[42] Leonhardt, F., Walther, R.: Wandartige Träger. Deutscher Ausschuss für Stahlbeton, Heft 178, Verlag Wilhelm Ernst und Sohn. Berlin 1966.

[43] Demorieux, J.M.: Essais de Traction-Compression sur Modèles d'Ame de Poutre en Béton Armé. Annales de l'Institut Techn. du Bâtiment et des Travaux Publics, Juin 1969, No. 258, Juin 1970, No. 270.

[44] Paulay, T.: The Coupling of Shear Walls. Thesis. University of Canterbury, Christchurch, New Zealend, 1969.

\section{Methode der finiten Elemente}

[45] Schäfer, H., Link, J., Mehlhorn, G.: Zur wirklichkeitsnahen Berechnung von Stahlbetonplatten mit der Finite-Element-Methode. Beton- und Stahlbetonbau 11/1975. Verlag Wilhelm Ernst und Sohn, Berlin.

[46] Cervenka, V.: Inelastic Finite Element Analysis of Reinforced Concrete Panels under In-plane Loads. Thesis. University of Colorado, 1970.

[47] Anderheggen, E.: Finite Element Analysis Assuming Rigid-Ideal-Plastic Material Behavior. Institut für Baustatik und Konstruktion, ETH Zürich, Bericht $\mathrm{Nr}$. 70, Birkhäuser Verlag Basel und Stuttgart, 1977. 
D

F

$F_{x}, F_{y}$

$F_{B}$

$F_{0}$

H

$K(\xi)$

L

$L_{u}, L_{0}$

$L_{d}$

$\mathrm{L}$

$M ; M_{y}, M_{z}$

N

$N_{x}, N_{y}, N_{x y}$

$P_{x}, P_{y} ; P_{s} ; P_{\xi}, P_{n}$

$P_{v}, P_{h}, P_{s}, P_{u}$

$P_{y}^{L}, P_{y}^{R}$

$P_{b}$

Q

$Q_{D}, Q_{B}$

Qff

$R_{x}, R_{y}, R_{x y}$

5

$\bar{s}$

$s_{s}, s_{u}$

T

U, V, w

Z

$Z_{f}$

$z_{u}, z_{f u} ; z_{0}, z_{f o}$

$\bar{z}_{0}, \bar{z}_{f o}$
Druckkraft

Querschnittsfläche; Spannungsfunktion

Querschnittsfläche eines Armierungseisens in $x$-bzw. $y$-Richtung

Querschnittsfläche eines Bügels

vam Schubfluss umflossene Fläche

Stützfunktion eines konvexen Körpers

Funktion $\cot 2 \alpha(\xi)$

Länge

horizontale Länge der Trajektorien $\alpha_{u}$ bzw. $\alpha_{0}$

Dissipationsleistung

Leistung der äusseren Kräfte

Biegemomente

Normalkraft

Membrankräfte der Stahlbetonscheibe (pro Längeneinheit)

Fliesskräfte (pro Längeneinheit) der Armierung in $x_{-}^{-}, y^{-}, s^{-}$, $\xi-, \quad n$-Richtung

Bügelfliesskräfte (pro Längeneinheit) in vorderer, hinterer, seitlicher, unterer Querschnittsseite

$P_{y}$ links bzw. rechts des Querschnittes

Bruchwiderstand ( $p r o$ Längeneinheit) des Betons

Querkraft

von der Druckdiagonale bzw. von den Bügeln übertragene Querkraft

effektive Querkraft bei Vouten

Abkürzungen gemäss Anhang $A$

Schubfluss

Schubflussverteilung am Querschnitt ohne Resultierende

Schubfluss in seitlicher bzw. unterer und oberer Querschnittsseite

Torsionsmoment

Verschiebungen bzw. Verschiebungsgeschwindigkeiten

Zugkraft

Zugfliesskraft

Zugkraft bzw. Zugfliesskraft in unterem bzw. oberem Flansch

Horizontalkomponente von $z_{0} b z w . z_{\text {fo }}$ bei Vouten 


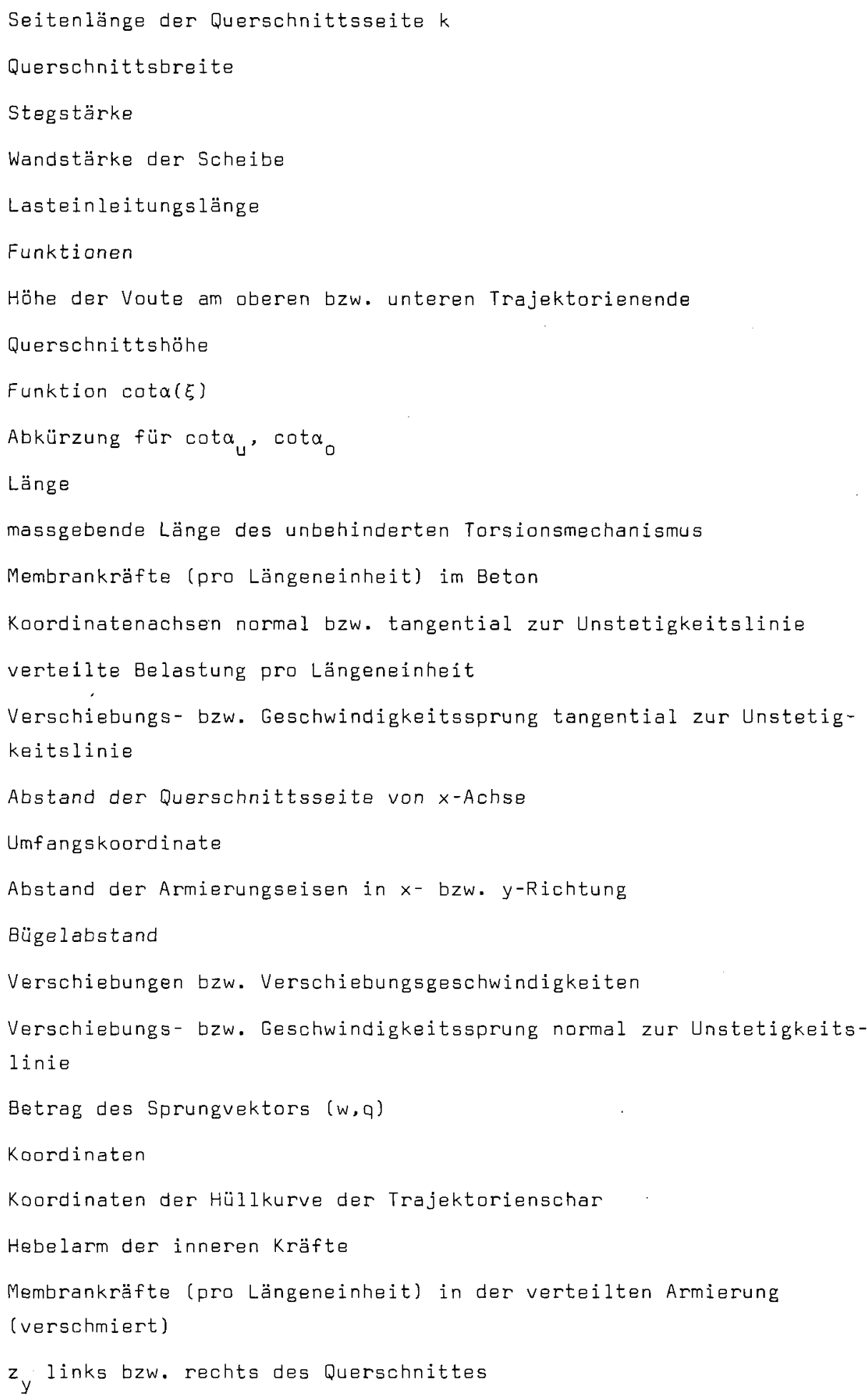

$\alpha$

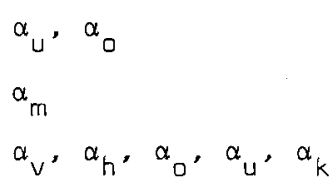




$\alpha_{d i m}$
$\alpha_{\square}$
$\alpha_{K}$
$\beta$
$\beta_{p}$
$\beta_{p s}, \beta_{p d}$
$\gamma, \gamma_{y}, \gamma_{z}, \bar{\gamma}$
$\gamma$
$\delta$

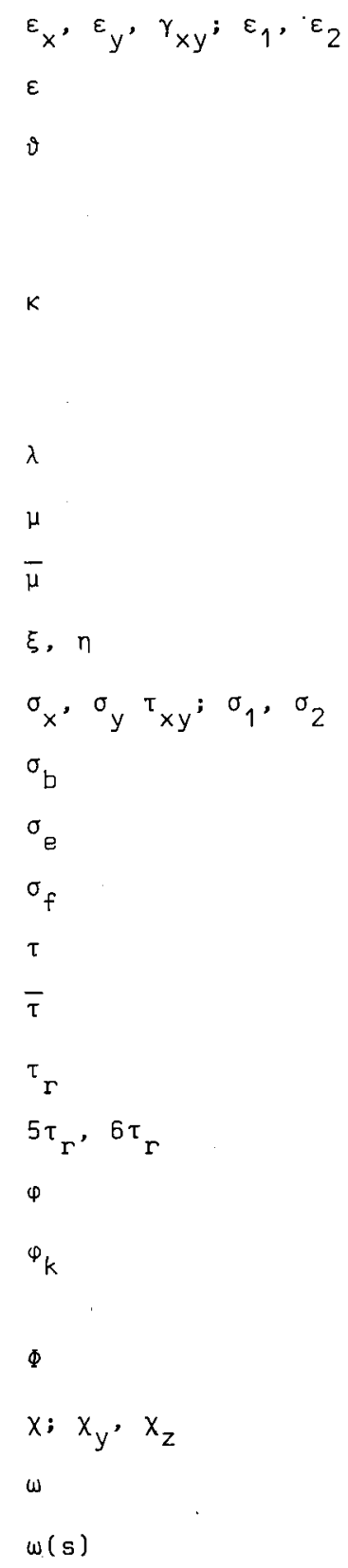

zur Dimensionierung der Armierung gewählte Trajektorienneigung Druckdiagonalenneigung kritische Trajektorienneigung $\left(\sin ^{2} \alpha_{K}=P_{y} / P_{b}\right)$ Neigung der Unstetigkeitslinie bezüglich $x$-Achse; Neigung der Rotationsachse des Torsionsmechanismus bezüglich $x$-Achse

Betonprismenfestigkeit

statische bzw. dynamische Betonprismenfestigkeit

verallgemeinerte Verformungen 'Schiebung der Balkenachse'

Voutenanzug; Neigung der Diagonale eines wandartigen Trägers

Neigung des Sprungvektors $(w, q)$ bezüglich der Normalen zur Unstetigkeitslinie; Neigung der Aussennormalen einer Querschnittsseite bezüglich $y$-Achse

Verzerrungen in Membranebene

verallgemeinerte Verformung 'Verlängerung der Balkenachse'

verallgemeinerte Verformung 'Verdrehung pro Längeneinheit der Balkenachse'; Neigung der Senkrechten zum Sprungvektor $(w, q)$ bezüglich $x$-Achse

Reduktionsfaktor für Betonfestigkeit und Wandstärke; Verhältnis der Werte von $\left(P_{y}+p\right)$ am unteren und oberen Drucktrajektorienende (Abschnitt 10.2)

Proportionalitätsfaktor im Fliessgesetz

Armierungsgehalt; Lagrange Multiplikator

bezogener Armierungsgehalt $\bar{\mu}=\mu \sigma_{f} /\left(k \beta_{p}\right)$

Koordinaten

Spannungskomponenten

Betondruckspannung

Stahlspannung

Stahlfliessspannung

nominelle Schubspannung

bezogene nominelle Schubspannung $\bar{\tau}=\tau /\left(K \beta_{p}\right)$

nominelle Rissschubspannung [29]

obere nominelle Schubspannungsgrenzen [29]

Verdrehung der Balkenachse

Starrkörperrotation des Scheibenstücks $k$ zwischen zwei Kollapsrissen

Fliessfläche; plastisches Potential; Winkel der inneren Reibung verallgemeinerte Verformungen 'Krümmung der Balkenachse'

Rotation

Einheitswölbung 


\section{Leere SeitelnBlank}


ANHANG A:

Ableitung der Fliessbedingungen (2.7) aus ihrer Stützfunktion (2.5)

Im Ausdruck (2.5) für die der gesuchten Fliessfigur zugeordnete Stützfunktion bzw. spezifische Dissipationsleistung treten die Dehnungsgeschwindigkeiten $\dot{\varepsilon}_{x}, \dot{\varepsilon}_{y}, \dot{\varepsilon}_{1}$, $\dot{\varepsilon}_{2}$ zwischen Absolutstrichen auf, und es müssen die folgenden sechs fälle unterschieden werden
1a) $\dot{\varepsilon}_{1} \geq 0, \dot{\varepsilon}_{2} \geq 0$
1b) $\quad \dot{\varepsilon}_{1} \leq 0, \dot{\varepsilon}_{2} \leq 0$
2a) $\dot{\varepsilon}_{x} \geq 0, \dot{\varepsilon}_{y} \geq 0$
2b) $\dot{\varepsilon}_{x} \leq 0, \dot{\varepsilon}_{y} \leq 0$
\}$\quad \dot{\varepsilon}_{1} \geq 0, \quad \dot{\varepsilon}_{2} \leq 0$
3a) $\dot{\varepsilon}_{x} \geq 0, \dot{\varepsilon}_{y} \leq 0$
3b) $\dot{\varepsilon}_{x} \leq 0, \dot{\varepsilon}_{y} \geq 0$
$\left(\dot{\varepsilon}_{1} \geq 0, \quad \dot{\varepsilon}_{2} \leq 0\right)$

Drückt man die Hauptdehnungsgeschwindigkeiten durch $\dot{\varepsilon}_{x}, \dot{\varepsilon}_{y}, \dot{\gamma}=\dot{\gamma}_{x y}$ aus und lässt von nun an einfachheitshalber die Punkte weg, so ist die stützfunktion $H\left(\varepsilon_{x}, \varepsilon_{y}, \gamma\right)$ in den sechs Definitionsbereichen gegeben durch

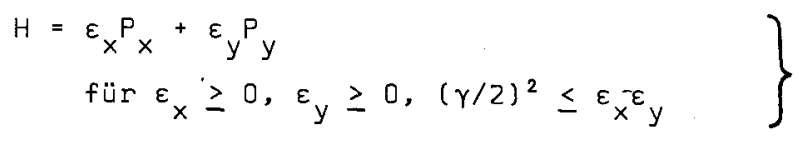

$$
\begin{aligned}
& \left.\begin{array}{rl}
H= & -P_{b}\left(\varepsilon_{x}+\varepsilon_{y}\right) \\
& \left.f \ddot{r} r \varepsilon_{x} \leq 0, \varepsilon_{y} \leq 0,(\gamma / 2)^{2} \leq \varepsilon_{x} \varepsilon_{y} \quad\right\}
\end{array}\right\} \\
& \left.\begin{array}{rl}
H= & \varepsilon_{x} P_{x}+\varepsilon_{y} P_{y}-\frac{1}{2} P_{b}\left(\varepsilon_{x}+\varepsilon_{y}-\sqrt{\left.\left(\varepsilon_{x}-\varepsilon_{y}\right)^{2}+\gamma^{2}\right)}\right. \\
& f \ddot{r} \varepsilon_{x} \geq 0, \varepsilon_{y} \geq 0,(\gamma / 2)^{2} \geq \varepsilon_{x} \varepsilon_{y}
\end{array}\right\} \\
& H=-\frac{1}{2} P_{b}\left(\varepsilon_{x}+\varepsilon_{y}-\sqrt{\left.\left(\varepsilon_{x}-\varepsilon_{y}\right)^{2}+\gamma^{2}\right)}\right. \\
& \text { für } \varepsilon_{x} \leq 0, \varepsilon_{y} \leq 0,(\gamma / 2)^{2} \geq \varepsilon_{x} \varepsilon_{y} \\
& H=\varepsilon_{x} P_{x}-\frac{1}{2} P_{b}\left(\varepsilon_{x}+\varepsilon_{y}-\sqrt{\left(\varepsilon_{x}-\varepsilon_{y}\right)^{2}+\gamma^{2}}\right. \\
& \text { für } \varepsilon_{x} \geq 0, \varepsilon_{y} \leq 0,\left((\gamma / 2)^{2} \geq \varepsilon_{x} \varepsilon_{y}\right) \\
& H=\varepsilon_{y} P_{y}-\frac{1}{2} P_{b}\left(\varepsilon_{x}+\varepsilon_{y}-\sqrt{\left.\left.\varepsilon_{x}-\varepsilon_{y}\right)^{2}+\gamma^{2}\right)}\right. \\
& \text { für } \varepsilon_{x} \leq 0, \varepsilon_{y} \geq 0,\left((\gamma / 2)^{2} \geq \varepsilon_{x} \varepsilon_{y}\right)
\end{aligned}
$$

Dabei weisen die Hauptdehnungsgeschwindigkeiten für $(\gamma / 2)^{2} \leq \varepsilon_{x} \varepsilon_{y}$ gleiches, für $(\gamma / 2)^{2} \geq \varepsilon_{x} \varepsilon_{y}$ ungleiches Vorzeichen auf. Die jeweils mit a) und b) bezeichneten Fälle können im folgenden formal gemeinsam behandelt werden, da sich die Stützfunktionen nur in den einzelnen Koeffizienten unterscheiden, und sich ihre Definitionsbereiche durch Spiegelung an den Ebenen $\varepsilon_{x}+\varepsilon_{y}=0$ bzw. $\varepsilon_{x}-\varepsilon_{y}=0$ zur Deckung bringen lassen. 
Mit den Abkürzungen

$R_{x}=P_{x}-N_{x}, \quad R_{y}=P_{y}-N_{y^{\prime}} \quad R_{x y}=-N_{x y}$,

im Fall (A.1a) und

$R_{x}=P_{b}+N_{x}, \quad R_{y}=P_{b}+N_{y}, \quad R_{x y}=-N_{x y}$,

im Fall (A.1b) lautet die Ungleichung (2.6)

$R_{x} \varepsilon_{x}+R_{y} \varepsilon_{y}+R_{x y} \gamma \geq 0$

In beiden Fällen gilt (A.5) im Bereich

$\varepsilon_{x} \geq 0, \varepsilon_{y} \geq 0, \quad-\sqrt{\varepsilon_{x} \varepsilon_{y}} \leq \gamma / 2 \leq+\sqrt{\varepsilon_{x} \varepsilon_{y}}$

da der anders lautende Definitionsbereich im Fall (A.1b) in den Vorzeichen der ersten beiden Koeffizienten (A.4b) berücksichtigt ist.

Die Bedingungen, denen $R_{x}, R_{y}, R_{x y}$ genügen müssen, damit die Ungleichung (A.5) für alle Sätze von $\varepsilon_{x}, \varepsilon_{y}$, $\gamma$ im Bereich $(A .6)$ erfüllt ist, sind gemäss Abschnitt 2.2 die gesuchten Fliessbedingungen.

Die als Funktion von $\varepsilon_{x}, \varepsilon_{y}, \gamma$ aufzufassende linke Seite von (A.5) wird für festes $\varepsilon_{x}, \varepsilon_{y}$ an derjenigen der beiden Grenzen (A.6) für $\gamma$ minimal, an der gilt

$R_{x y} \gamma=-2\left|R_{x y}\right| \sqrt{\varepsilon_{x} \varepsilon_{y}}$

Einsetzen von (A.7) in (A.5) ergibt

$\left.\begin{array}{l}R_{x} \varepsilon_{x}+R_{y} \varepsilon_{y}-2\left|R_{x y}\right| \sqrt{\varepsilon_{x} \varepsilon_{y}} \geq 0 \\ \varepsilon_{x} \geq 0, \quad \varepsilon_{y} \geq 0 .\end{array}\right\}$

Setzt man $\varepsilon_{y}=0$ bzw. $\varepsilon_{x}=0$ folgen sofort die Bedingungen

$R_{x} \geq 0, \quad R_{y} \geq 0$

Längs eines Strahles $\varepsilon_{x}=\mu^{2} \varepsilon_{y}$ geht (A.B) über in

$\left(R_{x} \mu^{2}+R_{y}-2\left|R_{x y}\right| \mu\right) \varepsilon_{y} \geq 0$,

$\varepsilon_{y}>0, \mu \geq 0$ 
Der minimale Wert des Ausdruckes in der Klammer von (A.10) muss also positiv sein. Nullsetzen der Ableitung nach $\mu$ ergibt

$\mu=\frac{\left|R_{x y}\right|}{R_{x}}$,

und durch Einsetzen von (A.11) in den Klammerausdruck folgt unter Berücksichtigung von (A.9) die weitere Bedingung

$\left.\begin{array}{l}R_{x y}^{2} \leq R_{x} R_{y}, \\ \text { fur } R_{x} \geq 0, R_{y} \geq 0 .\end{array}\right\}$

2. Fliessbedingungen im Bereich der Stützfunktionen (A.3)

Mit den Abkürzungen

$R_{x}=N_{x}-P_{x}+\frac{1}{2} P_{b}, \quad R_{y}=-N_{y}-\frac{1}{2} P_{b}, \quad R_{x y}=N_{x y}$,

im Fall (A.3a) und

$R_{x}=-N_{x}-\frac{1}{2} P_{b}, \quad R_{y}=N_{y}-P_{y}+\frac{1}{2} P_{b}, R_{x y}=N_{x y}$,

im Fall (A.3b) lautet die Ungleichung (2.6)

$R_{x} \varepsilon_{x}-R_{y} \varepsilon_{y}+R_{x y} \gamma-\frac{1}{2} P_{b} \sqrt{\left(\varepsilon_{x}-\varepsilon_{y}\right)^{2}+\gamma^{2}} \leq 0$,

und muss erfüllt sein für alle

$\varepsilon_{x} \geq 0, \quad \varepsilon_{y} \leq 0$

Da das Vorzeichen von $\gamma$ frei ist und nur den dritten Term in (A.14) beeinflusst, ergibt sich die massgebende Ungleichung für diejenige Vorzeichenwahl, die zu positivem, drittem Term führt:

$R_{x y}^{\gamma}=\left|R_{x y}\right| \cdot|\gamma|$

Man stellt sofort fest, dass Ungleichung (A.14) für $\gamma=0$ wegen (A.9), (A.15) erfüllt ist, und man verliert deshalb keine Bedingung für $R_{x}, R_{y}, R_{x y}$, wenn $\gamma \neq 0$ angenommen wird.

Längs eines Strahles

$\varepsilon_{x}=\frac{1}{2}(\mu+\nu)|\gamma|, \quad \varepsilon_{y}=\frac{1}{2}(\mu-\nu)|\gamma|$,

geht die Ungleichung (A.14) mit (A.16) über in

$\left\{\frac{1}{2}\left(R_{x}-R_{y}\right) \mu+\frac{1}{2}\left(R_{x}+R_{y}\right) \nu+\left|R_{x y}\right|-\frac{1}{2} P_{b} \sqrt{1+\nu^{2}}\right\}|\gamma| \leq 0$. 
Der Gültigkeitsbereich $(A .15)$ ist in den neuen Variablen $\mu, \nu$ gegeben durch

$v \geq|\mu|, \quad$ oder

$-\nu \leq \mu \leq \nu \quad \nu \geq 0$

Die Funktion $F(\mu, v)$ in der geschweiften Klammer von (A.18) muss also an der Stelle ihres Maximums im Bereich (A.19) kleiner oder gleich null sein. Da F linear in $\mu$ ist, liegt das Maximum an derjenigen der beiden Grenzen (A.19) für $\mu$, an welcher gilt

$\frac{1}{2}\left(R_{x}-R_{y}\right) \mu=\frac{1}{2}\left|R_{x}-R_{y}\right| v$.

Mit der Abkürzung

$M=\frac{1}{2}\left(R_{x}+R_{y}\right)+\frac{1}{2}\left|R_{x}-R_{y}\right|=\operatorname{Max}\left(R_{x}, R_{y}\right)$

erhält man durch Einsetzen von (A.20) in (A.18)

$\left.\begin{array}{l}F(\nu)=M \nu+\left|R_{x y}\right|-\frac{1}{2} P_{b} \sqrt{1+\nu^{2}} \leq 0, \\ \text { für } \nu \geq 0 .\end{array}\right\}$

Es sind nun zwei Fälle zu unterscheiden: Ist $M \leq 0$, so liegt das Maximum der Funktion $F(v)$ an der Grenze von $v=0$, und es folgt sofort

$\left.\begin{array}{l}\left|R_{x y}\right| \leq \frac{1}{2} P_{b} \quad \text { bzw. } \quad R_{x y}^{2} \leq\left(\frac{1}{2} P_{b}\right)^{2}, \\ \text { für } M \leq 0 .\end{array}\right\}$

Ist $M \geq 0$ wird $F(v)$ für $\nu>0$ maximal. Nullsetzen der Ableitung nach $v$ ergibt

$M-\frac{1}{2} P_{b} \nu / \sqrt{1+\nu^{2}}=0$ :

$\nu=M /+\sqrt{\left(\frac{1}{2} P_{b}\right)^{2}-M^{2}}$.

Bei Beachtung der jeweils gültigen Abkürzungen (A.13) bzw. (A.4) verifiziert man leicht, dass der Ausdruck unter der Wurzel in (A.24) wegen (A.9) immer positiv ist und die Funktion $F(v)$ somit für $M \geq 0$ ein Maximum besitzt. Einsetzen von (A.24) in (A.22) ergibt

$\left.\begin{array}{l}R_{x y}^{2}+M^{2} \leq\left(\frac{1}{2} P_{b}\right)^{2}, \\ \text { für } M \geq 0 .\end{array}\right\}$

Auf die analoge Diskussion für den Bereich der Stützfunktionen (A.2) wird hier verzichtet, da sie keine neuen Fliessflächen mehr liefert, sondern nur noch bisher nicht erhaltene Grenzen zwischen den Fliessflächen. Diese kann man aber auch direkt aus den Fliessflächen ermitteln.

Mit den Ungleichungen (A.9), (A.12), (A.23) und (A.25) sind die elf Fliessbedingungen (2.7 I-XI) gewonnen: Einsetzen der Abkürzungen (A.4) in (A.9) bzw. (A.12) ergibt die Fliessbedingungen (2.7 VIII-XI) bzw. (2.7 I, II). Mit den Abkürzungen (A.13) erhält man aus (A.23) 
bzw. (A.25) die Fliessbedingungen (2.7 III) bzw. (2.7 IV-VII). Bei der Auswertung von (A.25) geht man dabei am besten so vor, dass man der Reihe nach $M$ gleich $R_{x} b z w$. $R_{y}$ setzt. Die für

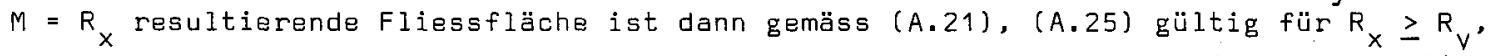

$R_{x} \geq 0$. Die noch fehlenden Gültigkeitsgrenzen erhält man durch Aufzeichnen der Fliessflächen oder mathematisch, wie erwähnt, durch Diskussion der Stützfunktion (A.2).

Greift man etwas vor und betrachtet Fig. 3.2, so ist sofort ersichtlich, dass die sechs Definitionsbereiche der Stützfunktion den sechs Kanten bzw. Ecken der Fliessfigur entsprechen, die Grenzen zwischen den Definitionsbereichen dagegen den dazwischen liegenden Fliessflächen. Die Diskussion der Stützfunktion für alle sechs Definitionsbereiche liefert deshalb jede Fliessfläche mehrmals, nämlich von jeder Kante bzw. Ecke her, die die Fliessfläche begrenzt. Damit ist auch begründet, warum die Diskussion der stützfunktion im Bereich (A.2) keine neuen Fliessflächen mehr liefert. 
ANHANG B: Rechenwerte zu Fig. 7.6

\begin{tabular}{|c|c|c|c|c|c|c|c|c|c|c|c|c|c|c|c|c|}
\hline Versuch & $\begin{array}{c}\text { Qu } \\
{[\text { to }]}\end{array}$ & $\underset{[\mathrm{cm}]}{H}$ & $\begin{array}{c}2 \\
{[\mathrm{~cm}]}\end{array}$ & $\begin{array}{c}b_{0} \\
{[\mathrm{~cm}]}\end{array}$ & $\begin{array}{c}1 \\
b_{m} \\
{[\mathrm{~cm}]}\end{array}$ & $\frac{a}{2}$ & $\begin{array}{c}\phi \\
{[\mathrm{mm}]}\end{array}$ & Anzahl & {$\left[\begin{array}{c}\text { Bügel } \\
\mathrm{F}_{\mathrm{B}} \\
{\left[\mathrm{cm}^{2}\right]}\end{array}\right.$} & {$\left[\begin{array}{c}t_{B} \\
{[\mathrm{~cm}}\end{array}\right]$} & $\left.\begin{array}{c}\sigma_{0.2} \\
{\left[10 \mathrm{~cm}^{2}\right.}\end{array}\right]$ & {$\left[\begin{array}{c}\beta_{p}^{3)} \\
{\left[\mathrm{kg} / \mathrm{cm}^{2}\right]}\end{array}\right.$} & $\bar{\tau}^{4)}$ & $\bar{\mu}^{5}$ & $\begin{array}{r}6) \\
\cot \alpha_{E x}\end{array}$ & $\begin{array}{c}\text { 7) } \\
\cot \alpha_{\text {th }}\end{array}$ \\
\hline T 1 & 80 & 90 & 75 & 10 & 8 & 3.35 & 12 & $\|$ & 4.16 & 8 & 4.35 & 242 & .55 & .65 & - & 1 \\
\hline T2 & 116 & 90 & 75 & 10 & 8 & 3.35 & 12 & II & 1.09 & 8 & 4.27 & 225 & .86 & .65 & 1.65 & 1.45 \\
\hline ET4 & 10.1 & 30 & 27 & 5 & 3.5 & 3.9 & 6 & $\|$ & .28 & 11 & 3.2 & 230 & .47 & .20 & 2.30 & 1.96 \\
\hline TP2 & 76 & 97 & 75 & 15 & 13 & 4.35 & 10 & $\|$ & .79 & 15 & 4.31 & 250 & .31 & .14 & 2.21 & 2.46 \\
\hline IP4 & 90 & 90 & 75 & 8 & 6 & 4.35 & 10 & $\|$ & .79 & 8.5 & 4.31 & 451 & .45 & .30 & 1.50 & 1.54 \\
\hline LT 2/\| & 93 & 97 & 75 & 15 & 13 & 4.35 & 10 & $\|$ & .79 & 15 & 4.54 & 289 & .33 & .13 & 2.68 & 2.60 \\
\hline
\end{tabular}

1) $b_{m}=b_{0}-$ Bügelüberdeckung beidseitig

2) $a=$ Schubspannweite $=$ Abstand Auflager - Einzellast

3) Lastschritte $\sim \beta_{p} / 8$, Losthalt 15 min pro Schrift
4) $\bar{\tau}=\frac{Q_{u}}{b_{m} 2 \beta_{p}} \quad$ 5) $\bar{\mu}=\frac{2 \cdot F_{B}}{b_{m}{ }^{\dagger}} \frac{\sigma_{02}}{\beta_{p}}$

6) $\cot \alpha_{E x}=\frac{\bar{\tau}}{\bar{\mu}}$ (Vert. bü.), $\cot \alpha_{E x}=\frac{2 \bar{\tau}}{\bar{\mu}}-1$ (Schrägb.)

7) $\sin \alpha_{\text {th }}=\sqrt{\mu} \quad " \quad, \sin \alpha_{\text {th }}=\sqrt{\bar{\mu} / 2}$

ANHANG B: Rechenwerte zu Fig. 7.7

\begin{tabular}{|c|c|c|c|c|c|c|c|c|c|c|c|c|c|c|c|c|}
\hline \multirow{3}{*}{$\begin{array}{l}\text { Versuch } \\
\text { CIL }\end{array}$} & \multirow{3}{*}{$\begin{array}{l}\text { Qu } \\
{[\text { to }]}\end{array}$} & \multicolumn{5}{|c|}{ Vertikalbügel, 2 -schnittig } & \multirow{2}{*}{\multicolumn{2}{|c|}{$\begin{array}{c}\left.\beta_{p d}\right|^{\beta_{p}} \beta_{p s} \\
{\left[\mathrm{~kg} / \mathrm{cm}^{2}\right]}\end{array}$}} & \multicolumn{2}{|c|}{ Fig. 7.70} & \multicolumn{2}{|c|}{ Fig. $7.7 \mathrm{~b}$} & \multicolumn{2}{|c|}{ Fig. $7.7 \mathrm{c}$} & \multicolumn{2}{|c|}{ Fig. $7.7 \mathrm{~d}$} \\
\hline & & $\stackrel{\phi}{\phi \mathrm{mm}]}$ & {$\left[\begin{array}{c}F_{B} \\
{\left[\mathrm{~cm}^{2}\right]}\end{array}\right.$} & $\begin{array}{c}{ }^{{ }_{B}} \\
{[\mathrm{~cm}]}\end{array}$ & $\begin{array}{c}\sigma_{0.2} \\
{\left[t_{0} / \mathrm{c}\right.}\end{array}$ & $\begin{array}{l}\theta_{\text {e.max }} \\
\left.m^{2}\right]\end{array}$ & & & $\bar{\tau}$ & $\bar{\mu}$ & $\bar{\tau}$ & $\bar{\mu}$ & $\bar{\tau}$ & $\bar{\mu}$ & $\bar{\tau}$ & $\bar{\mu}$ \\
\hline & & 6 & .31 & 12.5 & 4.95 & $5.7^{11}$ & & & .275 & .087 & .220 & .070 & .275 & .087 & .275 & .100 \\
\hline$C \backslash R$ & $3 t .8$ & 10 & .75 & 30 & 5.48 & 5.48 & & 300 & .250 & .097 & .200 & .077 & .250 & .097 & 250 & .097 \\
\hline$C 2 L$ & 37.1 & 6 & .31 & 12.5 & 4.95 & 5.9 & & חרת & .283 & .084 & .252 & .075 & 315 & .094 & .283 & 100 \\
\hline$C 2 R$ & 35.2 & 8 & .52 & 20 & 5.35 & 5.35 & 505 & $0<0$ & .268 & .095 & .238 & .085 & .298 & .106 & .268 & .095 \\
\hline C3L & 33.1 & 6 & .29 & 20 & 5.28 & 6.3 & & 250 & 291 & .061 & .285 & .060 & .355 & .075 & .291 & .073 \\
\hline$C 3 R$ & 28.6 & 8 & .49 & 30 & 5.37 & 5.7 & Jis & 250 & .253 & .069 & .247 & .068 & .310 & .085 & .253 & .074 \\
\hline C $4 \mathrm{~L}$ & 30.4 & 6 & .31 & 20 & 4.95 & 6.0 & & & .221 & .051 & .208 & .048 & .260 & .060 & .221 & .061 \\
\hline$C 4 R$ & 31.9 & 8 & .52 & 30 & 5.35 & 5.75 & Jou & $J<4$ & .233 & .061 & .219 & .058 & .273 & .072 & .233 & .066 \\
\hline
\end{tabular}

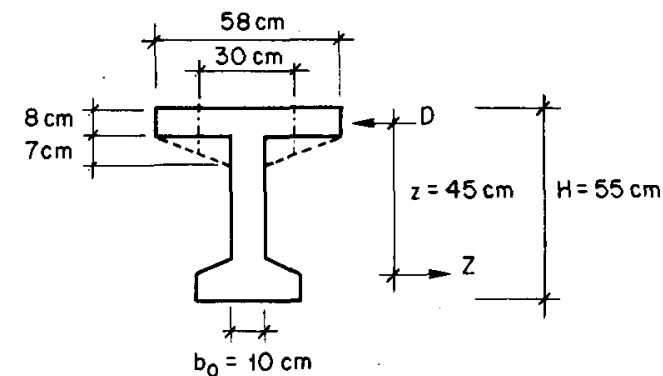

$-\mathrm{C}_{1}, \mathrm{C}_{2},-\cdots \mathrm{C}_{3},-.-\mathrm{C}_{4}$

Schubspannweite $150 \mathrm{~cm}$

Überdeckung Bügel beidseitig $1 \mathrm{~cm}$ :

$b_{m}=b_{0}-2 \mathrm{~cm}=8 \mathrm{~cm}$
1) entsprechend max. gemessener Bügeldehnung

2) kraftgesteuert, Kurzzeitversuch EMPA

3) dehnungsgesteuert, Dehnungsschritt $0.2 \cdot 10^{-3 / 2} \mathrm{~min}$ Dehnungshalt 2 min pro Schritt

$\bar{\tau}=\frac{Q_{u}}{b z \beta}, \bar{\mu}=\frac{2 \cdot F_{B}}{b_{B}^{1}} \quad \frac{\sigma}{\beta}$

Fig. $7.7 a: b=b_{m}, \quad \beta=\beta_{p d}, \sigma=\sigma_{0.2}$

Fig. $7.7 b: b=b_{0}, \beta=\beta_{\text {ps }}, \sigma=\sigma_{0.2}$

Fig. $7.7 \mathrm{c}: \quad b=b_{m}, \quad \beta=\beta_{\mathrm{ps}}, \sigma=\sigma_{0.2}$

Fig. $7.7 d: b=b_{m}, \beta=\beta_{p d}, \sigma=\sigma_{e, \text { max }}$ 